\title{
Multi-Scale Thermohydrologic Model Sensitivity-Study Calculations in Support of the SSPA
}

L. G. Glascoe, T. A. Buscheck, G. A. Loosmore, Y. Sun

\section{December 20, 2001}




\section{DISCLAIMER}

This document was prepared as an account of work sponsored by an agency of the United States Government. Neither the United States Government nor the University of California nor any of their employees, makes any warranty, express or implied, or assumes any legal liability or responsibility for the accuracy, completeness, or usefulness of any information, apparatus, product, or process disclosed, or represents that its use would not infringe privately owned rights. Reference herein to any specific commercial product, process, or service by trade name, trademark, manufacturer, or otherwise, does not necessarily constitute or imply its endorsement, recommendation, or favoring by the United States Government or the University of California. The views and opinions of authors expressed herein do not necessarily state or reflect those of the United States Government or the University of California, and shall not be used for advertising or product endorsement purposes.

This work was performed under the auspices of the U. S. Department of Energy by the University of California, Lawrence Livermore National Laboratory under Contract No. W-7405-Eng-48.

This report has been reproduced directly from the best available copy.

Available electronically at http://www.doc.gov/bridge

Available for a processing fee to U.S. Department of Energy

And its contractors in paper from

U.S. Department of Energy

Office of Scientific and Technical Information

P.O. Box 62

Oak Ridge, TN 37831-0062

Telephone: (865) 576-8401

Facsimile: (865) 576-5728

E-mail: reports@adonis.osti.gov

Available for the sale to the public from

U.S. Department of Commerce

National Technical Information Service 5285 Port Royal Road

Springfield, VA 22161

Telephone: (800) 553-6847

Facsimile: (703) 605-6900

E-mail: orders@ntis.fedworld.gov

Online ordering: http://www.ntis.gov/ordering.htm

OR

Lawrence Livermore National Laboratory

Technical Information Department's Digital Library

http://www.llnl.gov/tid/Library.html 
MULTI-SCALE THERMOHYDROLOGIC MODEL SENSITIVITY-STUDY CALCULATIONS IN SUPPORT OF THE SSPA

CONTENTS

Page

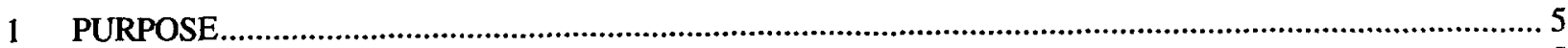

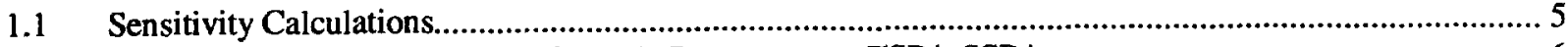

1.2 Calculations to Provide Thermodynamic Parameters to TSPA-SSPA ........................................................ 6

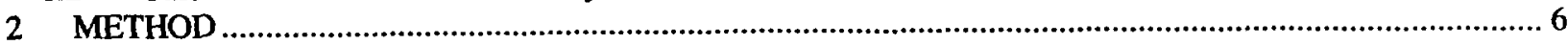

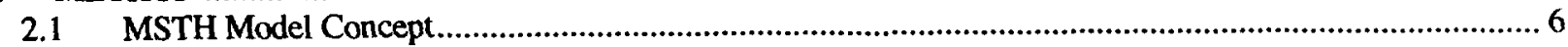

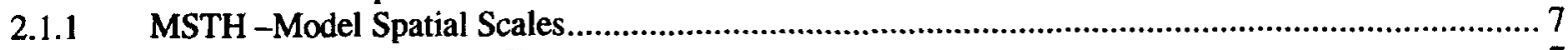

2.1.2 MSTH Model Submodel Types ..................................................................................................

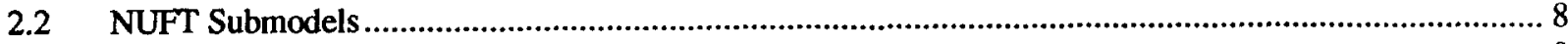

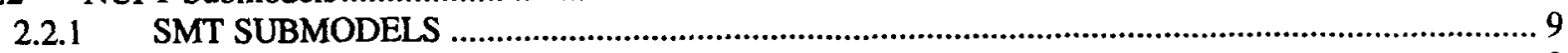

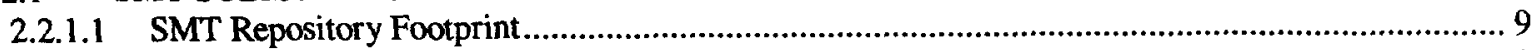

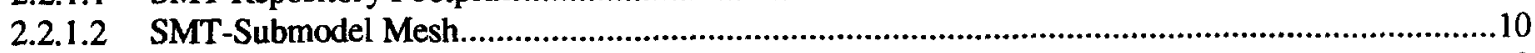

2.2.1.3 SMT-Submodel Boundary Conditions .....................................................................................

2.2.1.4 SMT-Submodel Heat Generation Rates..................................................................................

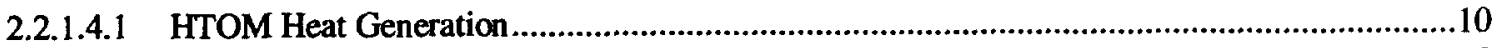

2.2.1.4.2 LTOM Heat Generation ..................................................................................................... 10

2.2.1.5 SMT-Submodel Material Properties..........................................................................................11

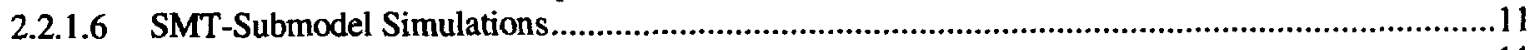

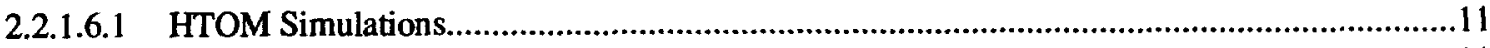

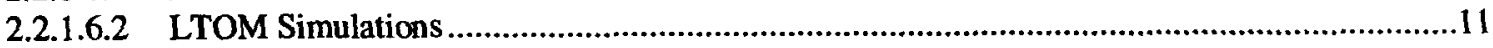

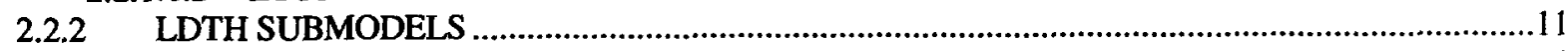

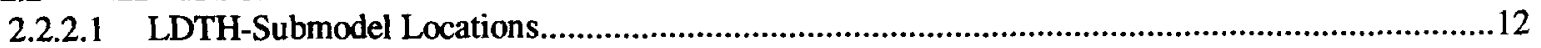

2.2.2.1.1 Stratigraphic Columns.................................................................................................12

2.2.2.1.2 Vertical Location of Repository Horizon ..........................................................................12

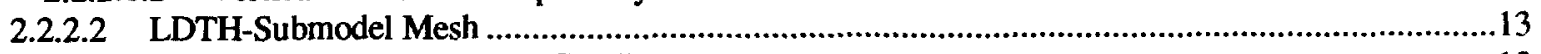

2.2.2.3 LDTH-Submodel Boundary Conditions ....................................................................................13

2.2.2.4 LDTH-Submodel Heat-Generation Rates ....................................................................................13

2.2.2.5 LDTH-Submodel Material Properties ..................................................................................... 14

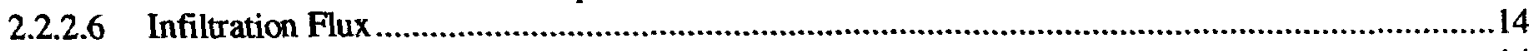

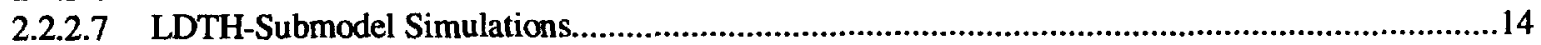

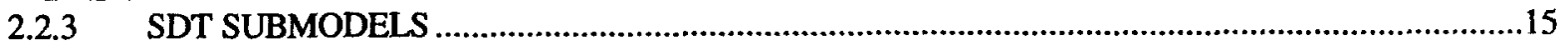

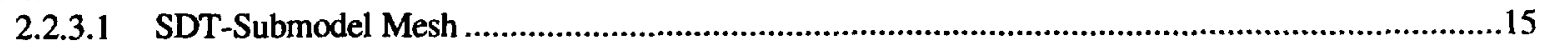

2.2.3.2 SDT-Submodel Boundary Conditions ......................................................................................15

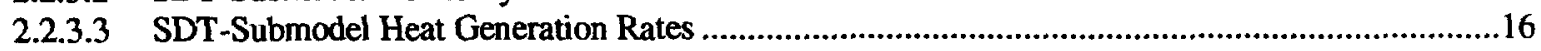

2.2.3.4 SDT-Submodel Material Properties ...........................................................................................16

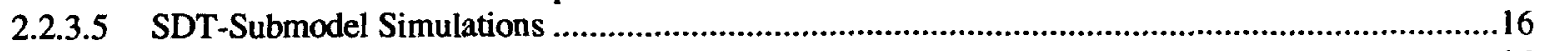

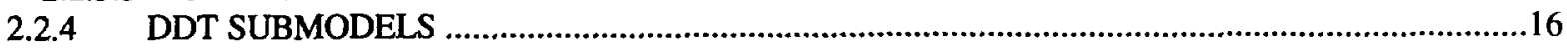

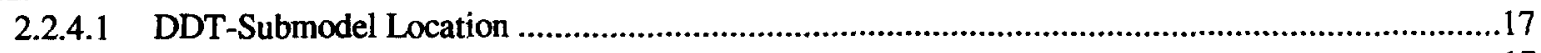

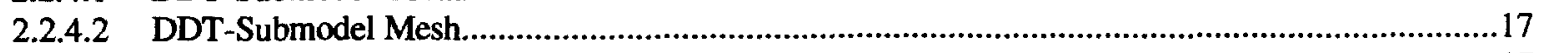

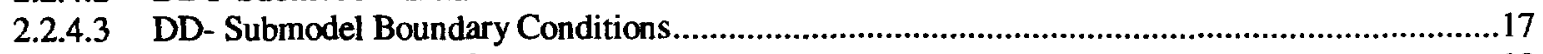

2.2.4.4 DDT-Submodel Heat Generation Rates..................................................................................18

2.2.4.5 DDT-Submodel Material Properties......................................................................................18

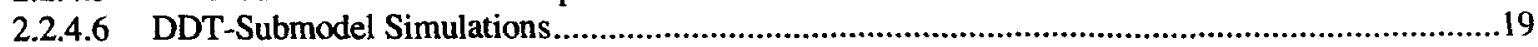

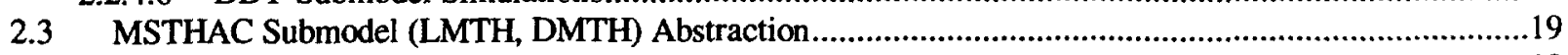

2.3.1 MSTHAC Step 1: Assemble Effective Host-Rock Areal Mass Loading, $A M L_{\text {effective }}$...........................19

2.3.2 MSTHAC Step 2: Interpolate LMTH-Model Temperatures..............................................................20

2.3.3 MSTHAC Step 3: Calculate DMTH-Model TH Variables.............................................................20

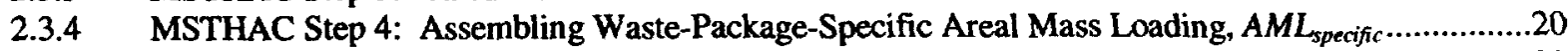

2.3.5 MSTHAC Step 5: Interpolate TH Variables for DMTH...................................................................20

2.3.6 MSTHAC Step 6: Determine Relative Humidity on the Drip Shield and Waste Package.................21 


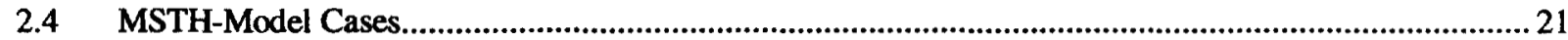

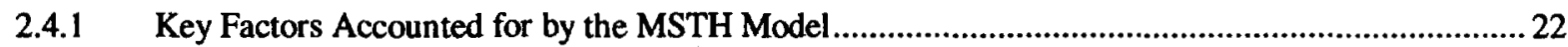

2.4.2 Total Submodel Simulations Per MSTH-Model Case .............................................................. 22

2.4.3 Accounting for the Influence of Drift Ventilation ....................................................................... 23

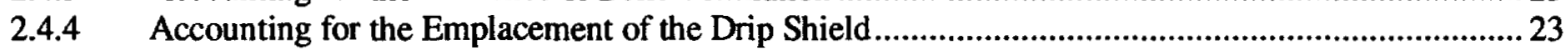

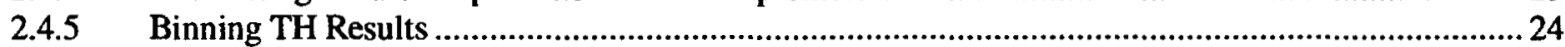

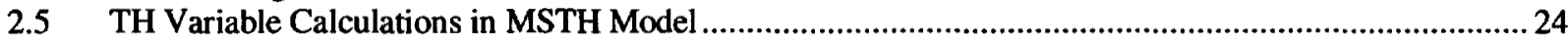

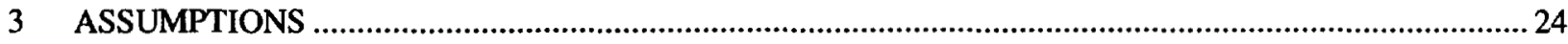

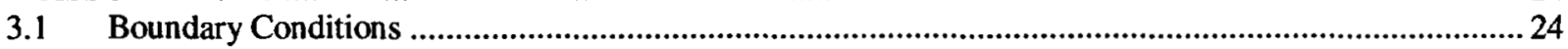

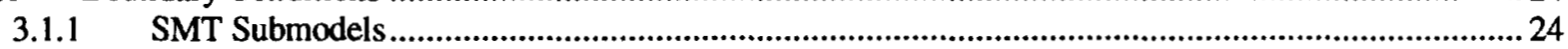

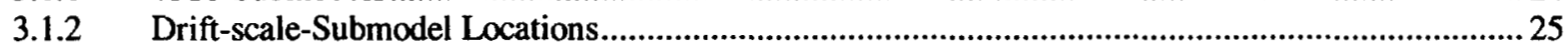

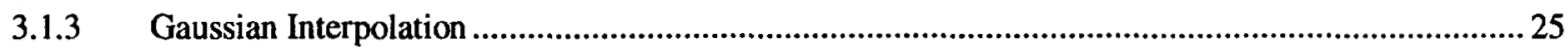

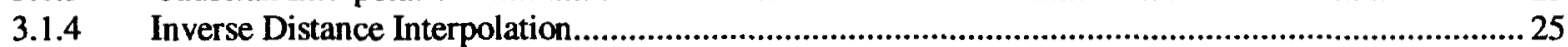

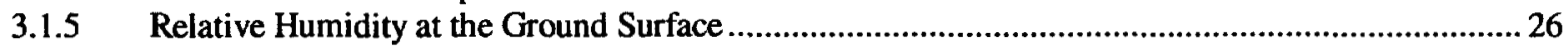

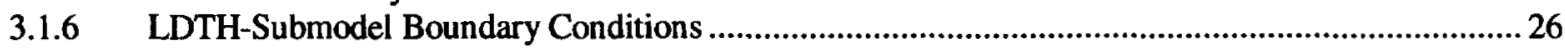

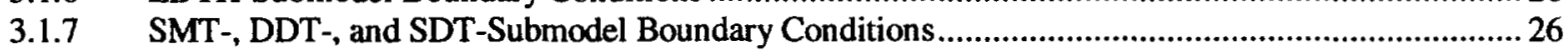

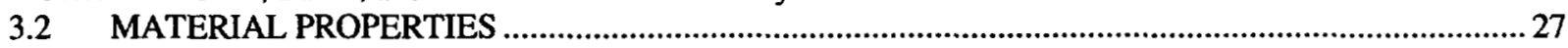

3.2.1 Permeability of the Drip Shield and Waste Package................................................................... 27

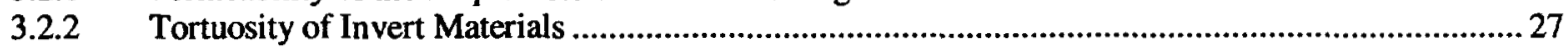

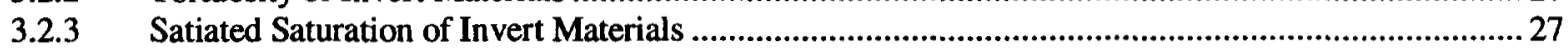

3.2.4 Thermal Conductivity Used in Conduction-Only Submodels .................................................... 27

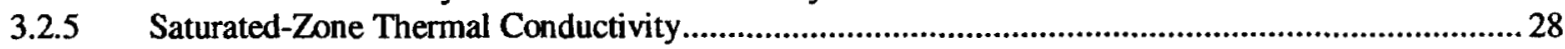

3.2.6 Thermal Conductivity and Density for the Active Fracture Model ...............................................28

3.2.7 Thermal Conductivity, the Lower and Upper Invert Layer ............................................................. 28

3.3 Heat Generation and Heat Transfer

3.3.1 Constant Heat Flux ..................................................................................................... 29

3.3.2 Effective Thermal Conductivity for Approximating the Influence of Thermal Radiation for DDT

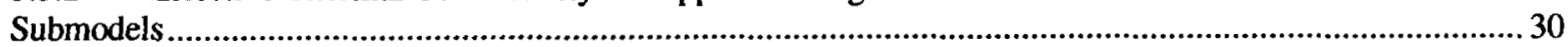

3.3.3 Effective Thermal Conductivity Approximating the Influence of Thermal Radiation In the Drift for

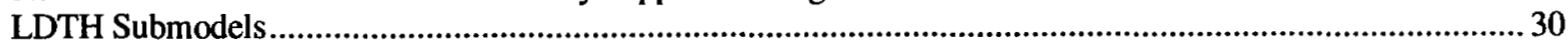

3.3.4 Effective Thermal Conductivity Approximating the Influence of Buoyant-Gas-Phase Convection On

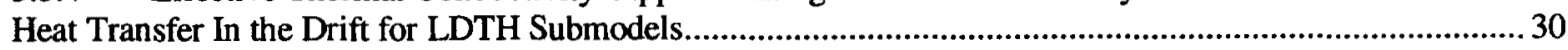

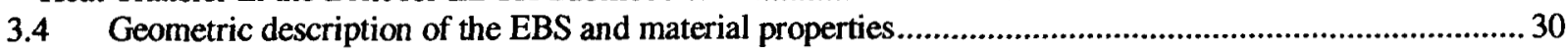

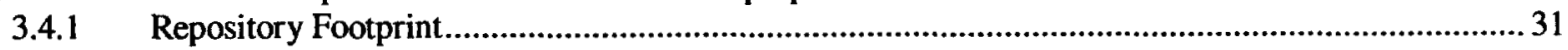

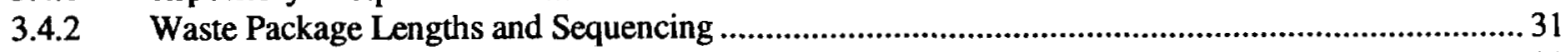

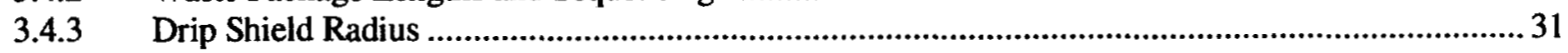

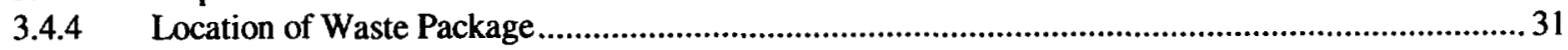

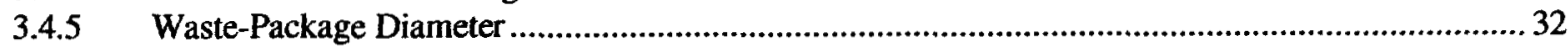

3.4.6 Gap Between Waste Package and Drip Shield ........................................................................... 32

3.4.7 Gap Between Waste Package and Invert.................................................................................... 32

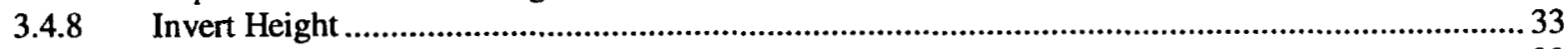

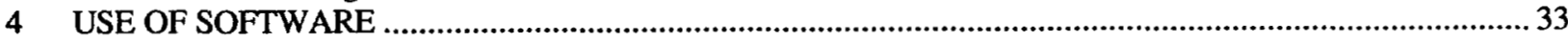

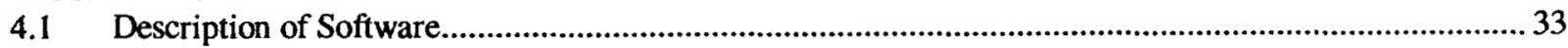

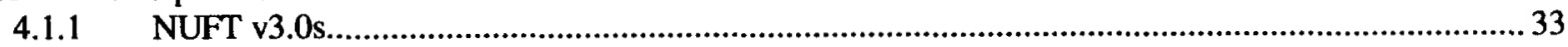

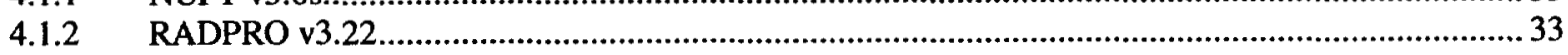

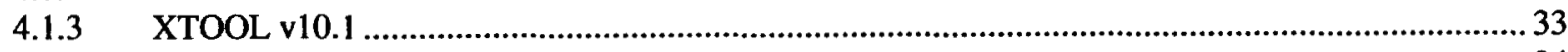

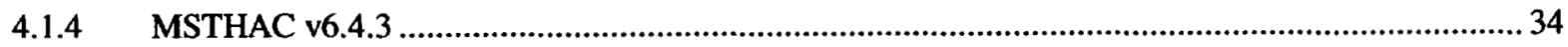

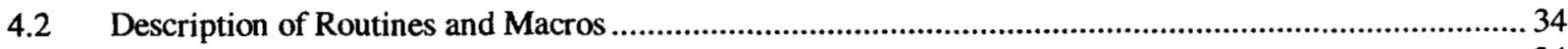

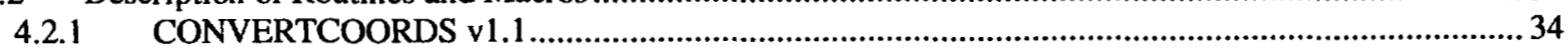

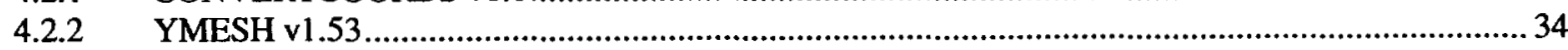

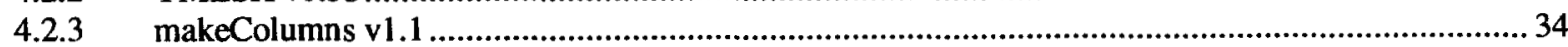

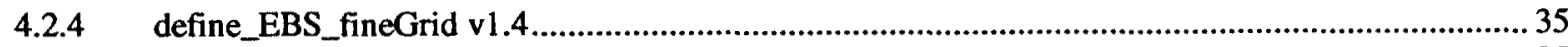

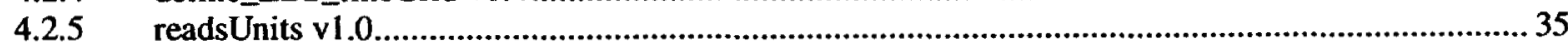

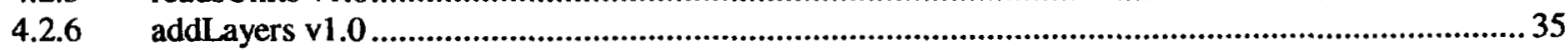

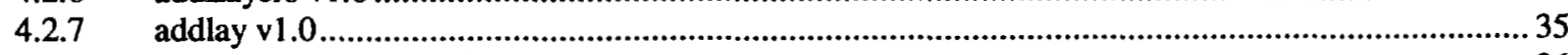

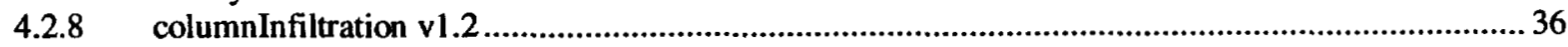




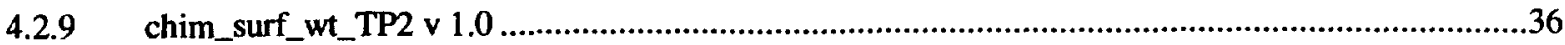

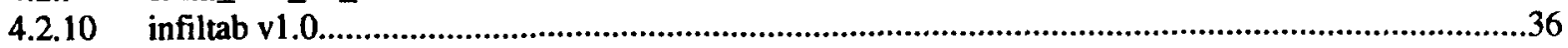

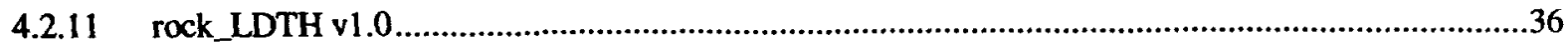

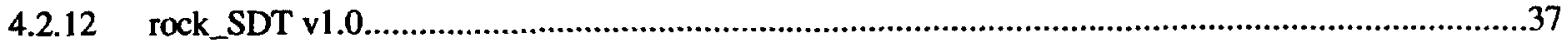

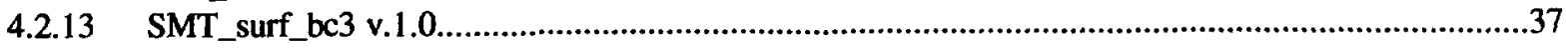

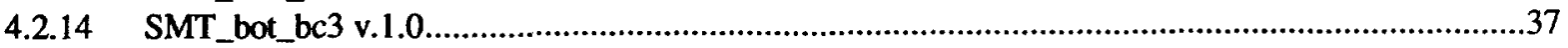

4.2.15 bound v1 0 .

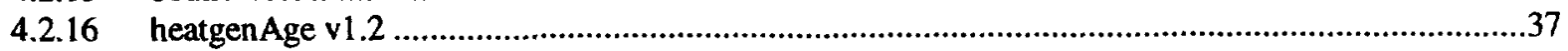

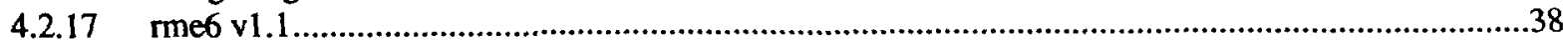

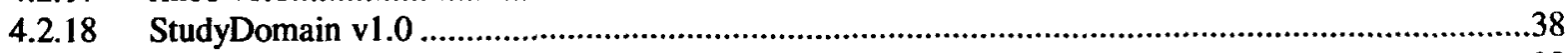

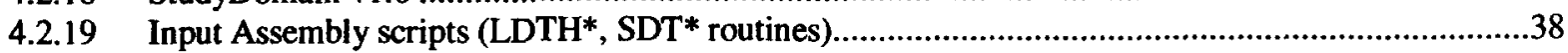

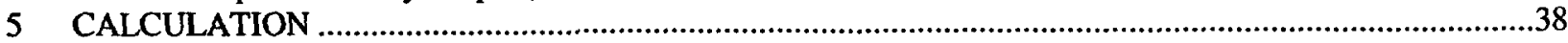

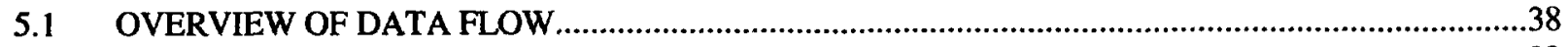

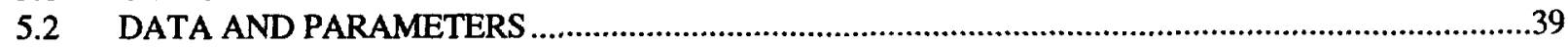

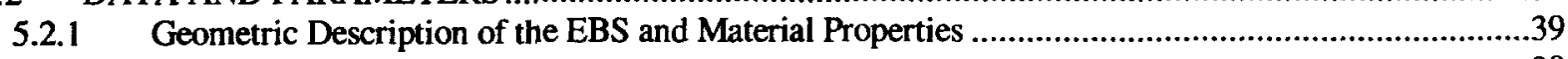

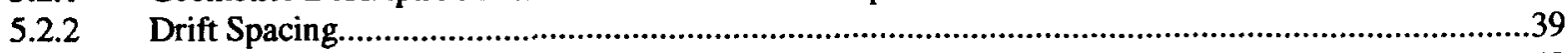

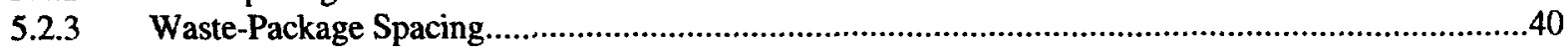

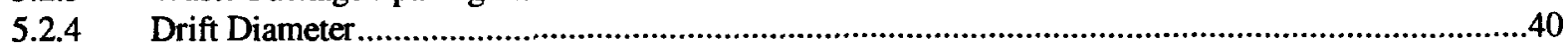

5.2.5 Properties of Air at Model Boundaries.......................................................................................

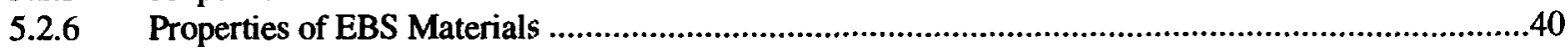

5.2.7 Hydrologic and Thermal Properties of Stratigraphic Units..............................................................40

5.2.8 Thermal Properties of Air Inside the Drifts ....................................................................................41

5.2.9 Thermal Properties of Stratigraphic Units for SMT Submodels .....................................................41

5.2.10 Effective Thermal Conductivity of Cavities Inside Drifts ................................................................41

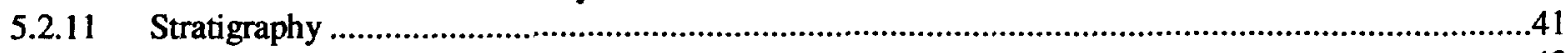

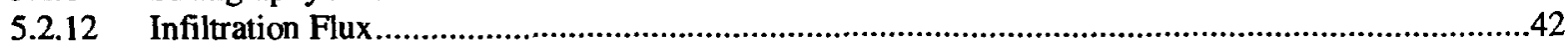

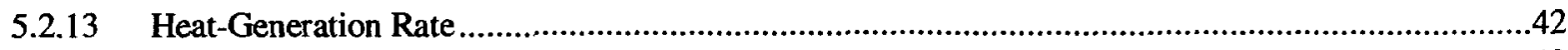

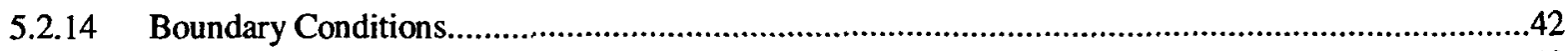

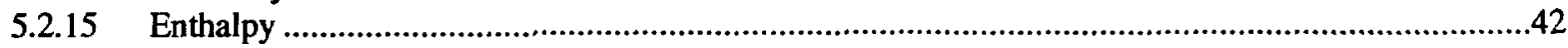

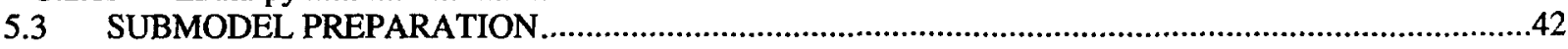

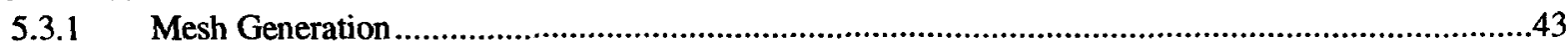

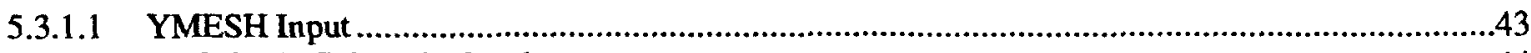

5.3.1.2 Drift-Scale-Submodel Meshes ..............................................................................................44

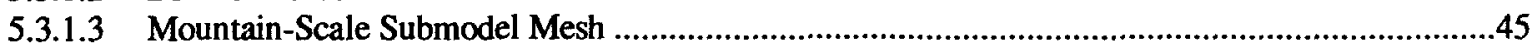

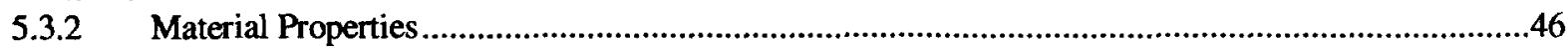

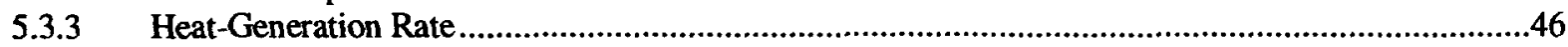

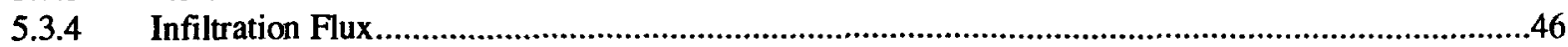

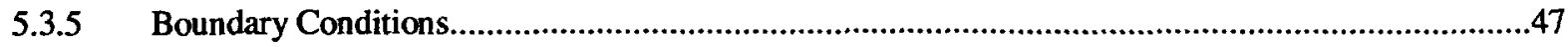

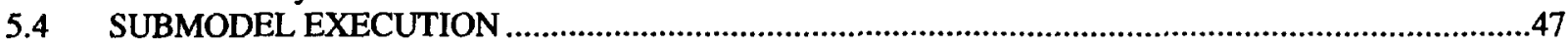

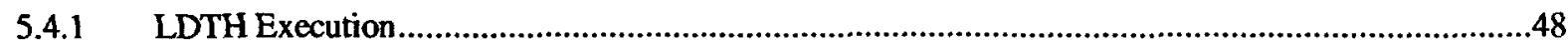

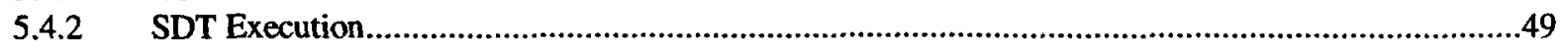

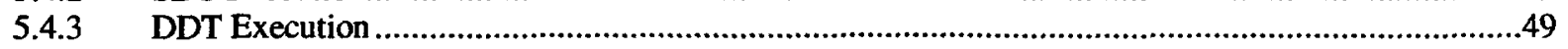

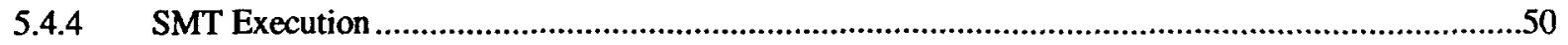

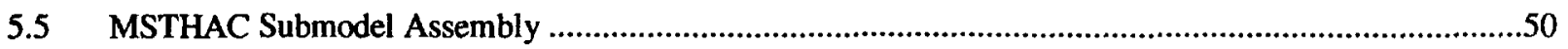

5.5.1 Stage1: Extract TH data from the all pre-closure submodels..................................................50

5.5.2 Stage 2: Abstract with MSTHAC TH data from the all pre-closure submodels..............................51

5.5.3 Stage 3: Extract TH data from the all post-closure submodels....................................................51

5.5.4 Stage 4: Abstract with MSTHAC TH data from the all post-closure submodels. ............................51

5.5.5 Stage 5: Combine the pre-closure and post-closure abstracted TH data. .......................................51

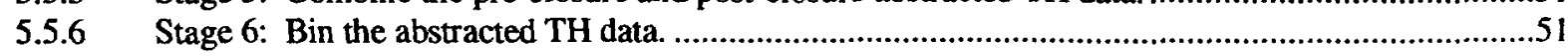

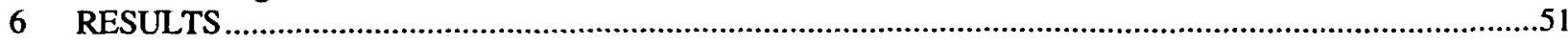

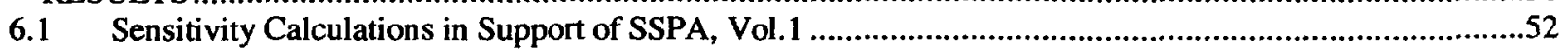

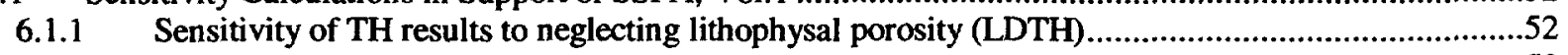

6.1.2 Impact of mountain-scale buoyant gas-phase convection on temperature (LDTH) ..........................52

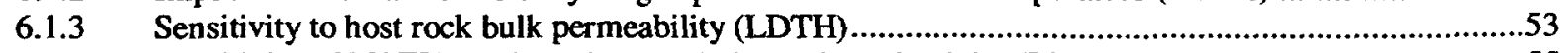

6.1.4 Sensitivity of MSTH results to host rock thermal conductivity (LDTH) .........................................55 
6.1.5 Sensitivity of MSTH Model Results to Lithophysal Porosity (LDTH) ............................................59

6.1.6 Sensitivity of TH to design and operational parameters (MSTH) ...................................................61

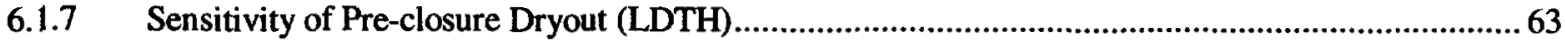

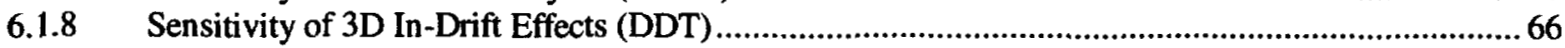

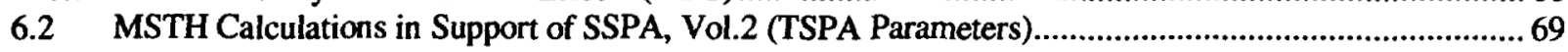

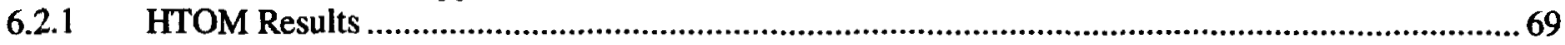

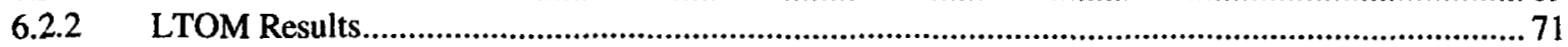

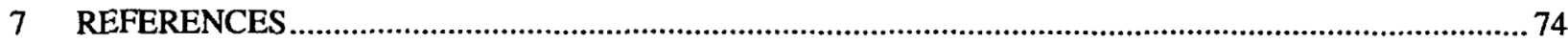

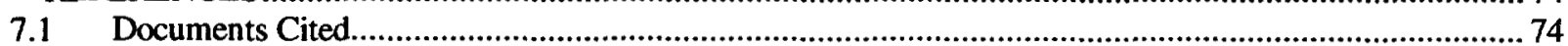

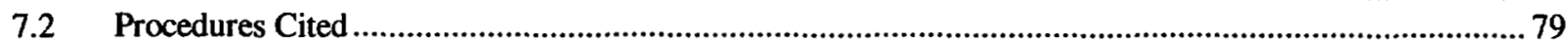

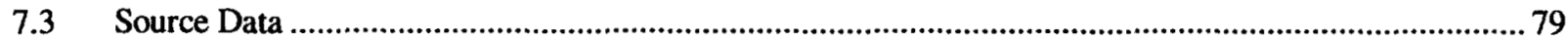

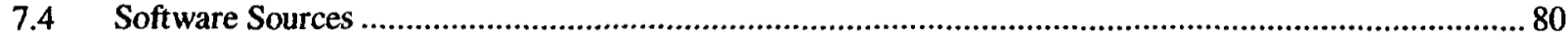

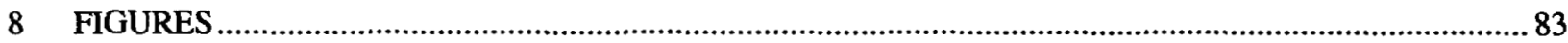

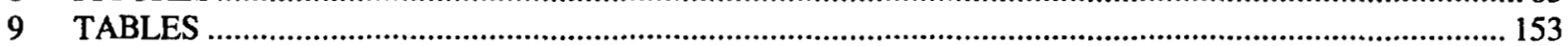




\section{PURPOSE}

The purpose of this calculation report is to document the thermohydrologic (TH) model calculations performed for the Supplemental Science and Performance Analysis (SSPA), Volume 1, Section 5 and Volume 2 (BSC 2001d [DIRS 155950], BSC 2001e [DIRS 154659]). The calculations are documented here in accordance with AP-3.12Q REV0 ICN4 [DIRS 154418]. The Technical Working Plan (TWP) for this document is TWP-NGRM-MD-000015 Rev01. These TH calculations were primarily conducted using three model types: (1) the Multiscale Thermohydrologic (MSTH) model, (2) the line-averaged-heat-source, drift-scale thermohydrologic (LDTH) model, and (3) the discrete-heat-source, drift-scale thermal (DDT) model. These TH-model calculations were conducted to improve the implementation of the scientific conceptual model, quantify previously unquantified uncertainties, and evaluate how a lower-temperature operating mode (LTOM) would affect the in-drift TH environment. Simulations for the higher-temperature operating mode (HTOM), which is similar to the base case analyzed for the Total System Performance Assessment for the Site Recommendation (TSPA-SR) (CRWMS M\&O 2000j [DIRS 153246]), were also conducted for comparison with the LTOM.

This Calculation Report describes (1) the improvements to the MSTH model that were implemented to reduce model uncertainty and to facilitate model validation, and (2) the sensitivity analyses conducted to better understand the influence of parameter and processs uncertainty. The METHOD Section (Section 2) describes the improvements to the MSTHmodel methodology and submodels. The ASSUMPTIONS Section (Section 3) lists the assumptions made (e.g., boundaries, material properties) for this methodology. The USE OF SOFTWARE Section (Section 4) lists the software, routines and macros used for the MSTH model and submodels supporting the SSPA. The CALCULATION Section (Section 5) lists the data used in the model and the manner in which the MSTH model is prepared and executed. And, lastly, the RESULTS Section (Section 6) lists the two calculations conducted for the SSPA (BSC 2001d [DIRS 155950], BSC 2001e [DIRS 154659]).

\subsection{Sensitivity Calculations}

Sensitivity analyses were performed to further evaluate and quantify the consequences of uncertainties in processes and model inputs. Eight areas of uncertainty were analyzed:

1. Sensitivity of TH results to the exclusion of vapor storage within the lithophysal cavities (porosity). Current models do not provide analyses of cavity storage separate from the overall fracture porosity in the DKM model (Section 6.1.1).

2. Sensitivity of the impact of mountain-scale buoyant gas-phase convection on temperature history (Section 6.1.2).

3. Sensitivity of MSTH model results to uncertainties in the bulk permeability of the host rock (Section 6.1.3). 
4. Sensitivity of MSTH model results to uncertainties in the host-rock thermal conductivity (Section 6.1.4).

5. Sensitivity of the TH models to the way lithophysal porosity affects other input parameters, such as bulk or host rock thermal conductivity (Section 6.1.5).

6. Sensitivity of the TH parameters to design and operational parameters (Section 6.1.6).

7. Sensitivity of pre-closure dry-out of near-field rock on post-closure in-drift temperature relative humidity due to ventilation (Section 6.1.8).

8. Sensitivity of three-dimensional in-drift effects (Section 6.1.8).

\subsection{Calculations to Provide Thermodynamic Parameters to TSPA-SSPA}

MSTH mdoel calculations were conducted to provide TH parameters required by the TSPASSPA (BSC 2001 [DIRS 154864]). The first set of MSTH model results pertain to the mean, upper and lower infiltration flux scenarios for the base-case HTOM (Section 6.2.1). The HTOM is similar to the TSPA-SR design case, but analyzed with updated models and operational

parameters. The second set of MSTH model results pertain to the mean, upper and lower infiltration flux scenarios for the LTOM where temperatures do not exceed approximately $85^{\circ} \mathrm{C}$ on the waste package (WP) surface (Section 6.2.2). Table 1 summarizes the operational parameters for the HTOM and the LTOM.

\section{METHOD}

The methodology discussed in this report include the MSTH model concept, the MSTH modeling system which includes building and executing the family of MSTH-model submodels, using NUFT 3.0s and the execution of the Multi-Scale Thermal-Hydrology Abstraction Code (MSTHAC). The control of the electronic management of data was accomplished for this calculation report in accordance to methods specified in the TWP (TWP-NGRM-MD-000015 Rev01).

\subsection{MSTH Model Concept}

Performance measures of the repository depend on TH behavior occurring at a scale of a few tens of centimeters around individual waste packages (WP) and emplacement drifts as well as on behavior at the repository (or mountain) scale. A single numerical model (e.g., embedding a 3-D drift-scale model with a relatively fine mesh into a 3-D mountain-scale model with a coarse mesh) would require an unfeasible number (millions) of grid blocks for a TH model. The MSTH model approach breaks the problem into more tractable pieces by superposing the results of 3-D mountain- and drift-scale thermal models onto those of two-dimensional drift-scale TH models. Thus, the MSTH-model approach divides the problem into 1-, 2-, and 3-D thermal and TH submodels. By dividing the problem, much more computationally efficient thermal conduction submodels can be used to address detailed 3-D heat-flow problems at the mountain and drift scales. The fundamental concept in the MSTH model is that the results from the 2-D LDTH submodels (see Section 2.1.2 below) can be modified to account for the influence of 3-D 
mountain-scale heat flow as well as for local deviations arising from waste-package-to-wastepackage variability in heat output.

\subsubsection{MSTH-Model Spatial Scales}

Two spatial scales are considered for the MSTH model: (1) the mountain scale (on the order of hundreds to thousands of meters) and (2) the drift-scale (on the order of tens of centimeters). Multiscale modeling must include interaction of processes at the drift-scale in the Engineered Barrier System (EBS) and the Near Field Environment (NFE) with processes at the scale of the mountain to properly account for effects such as faster cooling of waste packages and adjacent host rock near the repository edge, as compared to waste packages closer to the repository center. In addition to accounting for interaction of processes at the drift scale and mountain scale, the MSTH model also allows for consideration of the influence of waste-package-to-waste-package variability in heat output on TH behavior. The waste packages inventory consists of two major waste package categories: (1) Commercial Spent Nuclear Fuel (CSNF) waste packages, and (2) High Level Waste (HLW). The CSNF waste packages generate much more heat than the HLW waste packages. Moreover, there can be considerable variability in heat output among various types of CSNF waste packages, depending on whether the waste comes from pressurized-water reactors (called PWR waste packages) or waste from boiling-water reactor (called BWR waste packages) as well as on the age of the waste package (i.e., how long it has been since it was removed from the reactor core).

\subsubsection{MSTH Model Submodel Types}

The MSTH model simulates processes under a range of heat loading conditions to capture the edge effects within the repository and the discrete nature of waste packages. MSTH simulates at various locations within the domain to account for variations in stratigraphy and local infiltration flux. This MSTH-model calculation process involves solving six 'submodels' at different spatial scales. Four of these submodels are NUFT submodels (SMT, SDT, DDT, LDTH) which are input to MSTHAC submodels and two of these submodels (LMDTH, DMTH) are output from MSTHAC. A consistent naming convention is used for these submodels. The first letter describes the Dimensionality of the Heat-Source Approximation, where $\mathbf{S}$ stands for a 'smeared' heat source, $\mathbf{L}$ stands for a 'line-averaged' heat source, and D stands for a 'discrete' heat source. The second letter applies to the Spatial Scale where $M$ stands for 'mountain' scale and D stands for 'drift' scale. The last one or two letters refer to the Physical Processes Considered, where $\mathbf{T}$ means that 'thermal conduction and radiation' variables only are considered and TH means that all 'thermal-hydrological' processes are considered.

The four different NUFT submodels are run at different spatial scales. These four submodels are the following:

SMT (3-D Smeared-heat-source, Mountain-scale, Thermal-conduction) Submodel LDTH (2-D Line-averaged-heat-source, Drift-scale, Thermohydrologic) Submodel SDT (1-D Smeared-heat-source, Drift-scale Thermal-conduction) Submodel DDT (3-D Discrete-heat-source, Drift-scale Thermal-conduction) Submodel 
The results of the submodels are integrated with a tool called the Multiscale Thermohydrologic Abstraction Code (MSTHAC). For this report, MSTH model, the LDTH and SDT submodels were run at 33 geographic locations uniformly distributed over the repository area; these submodels use the stratigraphy, overburden thickness, TH boundary and infiltration fluxes appropriate for each location. At each of those 33 geographic locations, the LDTH- and SDTsubmodel calculations were conducted at four different values of Areal Mass Loading (AML, expressed in MTU/acre). The LDTH submodel domain is a 2-D drift-scale cross-section extending from the ground surface to the water table. The LDTH submodels are the only submodels to include coupled TH processes; these submodels assume a heat-generation history that is effectively that of a line-averaged waste package averaged for the entire waste-package inventory in the repository. The SDT submodels utilize the same heat-generation history as the LDTH submodels except that it is areally smeared over the repository plane in the SDT submodels.

In the MSTHAC execution the MSTH model generates the following two models as output:

LMTH (3-D Line-averaged-heat-source, Mountain-scale, Thermohydrologic) model: the intermediary model in the MSTH submodel calculation sequence.

DMTH (3D Discrete-heat-source, Mountain-scale, Thermohydrologic) model: the final model in the MSTH model calculation sequence.

Figure 1 illustrates the general conceptual relation between the four NUFT submodels (identified by red text) and the two MSTHAC models (identified by blue text). The successive nature of the NUFT submodel execution followed by the MSTHAC calculation for final output is illustrated in the flowchart of Figure 2.

\subsection{NUFT Submodels}

At each of 33 locations spaced evenly throughout the repository area, a 2-D LDTH submodel and a corresponding 1-D SDT sumodel are executed. The LDTH sumodels account for TH processes, including the influence of the local percolation flux (which is assumed to be equal to the local infiltration flux) and the local thermal and hydrologic properties. At each of the 33 geographic locations the LDTH and SDT sumodel calculations are performed at four different values of Areal Mass Loading (AML, expressed in MTU/acre). The different AMLs, are achieved by adjusting the spacing between emplacement drifts in the respective models. The highest AML is $20 \%$ larger than the nominal AML to account for regions of the repository that are hotter than nominal. The second highest AML is at the nominal AML value (e.g., 55 MTU/acre for the HTOM). The lowest two AMLs are 50 and 25 percent of the nominal AML value; the lower AMLs are required to account for the edge-cooling effect and/or cooler wastepackge locations (e.g., HLW waste packages).

The 3-D SMT and the 1-D SDT submodels share the same smeared-heat-source approximation and thermal-conduction-only representation of heat flow. The 1-D SDT submodels provide linkage between the SMT and the LDTH submodels. This linkage allows for the SMT submodel temperature to be "corrected" for both the influence of TH processes on temperature and for the 
influence of 2-D drift-scale dimensionality (orthogonal to the axis of the drift) of the emplacement drift and the waste-package heat source. The SMT, SDT, and LDTH submodels all share a blended heat-generation history of the entire WP inventory in the repository, which is normalized for a 1-m interval of an emplacement drift; hence, the heat-generation history is effectively that of an "average" WP.

The 3-D DDT submodel is a drift-scale submodel that includes individual waste packages of distinct heat-generation history. The DDT submodel solves for thermal conduction and accounts for thermal radiation in addition to thermal conduction between the waste packages, drip shield and drift surfaces (i.e., drift wall and floor).

The NUFT submodels are executed simultaneously prior to MSTHAC calculations (see Figure 2). The detailed calculation relationship between the submodels is outlined in the flowchart of Figure 3. Note the reference to 'Subflow' in each box of Figure 3. These refer to the sixteen detailed sub-flowcharts. Figure 4 provides a legend for these sixteen sub-flowcharts, while Figure 5 through Figure 20 are the sixteen successive sub-flowcharts, 'Subflow 1' through 'Subflow 16'. Note that the connections between the Sub-flowcharts are listed at the bottom of each figure. Also note that the list of Tracked Data Stores A, B, C, D, E, F, G, H, and I are listed in Table 2. Operations are described Table 4 through Table 10.

The details of the each submodel and the corresponding sub-flowcharts are discussed in the subsections below. The meshing, boundaries, materials and heat generation functions of each of the four NUFT submodels are discussed in detail in these subsections.

\subsubsection{SMT SUBMODELS}

The 3-D SMT submodel is used to determine the repository-scale variations in host-rock temperature $T$ resulting from the heat output from the entire inventory of 70,000 MTU of waste, including 63,000 MTU of CSNF waste packages and 7000 MTU of HLW waste packages. The SMT submodel includes the influence of mountain-scale thermal-property distribution, the edgecooling effect, which results from lateral heat loss at the repository edges, and the distribution of the overburden-thickness, i.e., the distribution of the depth of the repository horizon below the ground surface. The SMT submodel domain extends from the ground surface to $1000 \mathrm{~m}$ below the present-day water table and the lateral (adiabatic) boundaries are far enough away from the repository as not to affect repository temperatures. The temperature $1000 \mathrm{~m}$ below the water table is found by extrapolation using the routine Bound (Section 4.2.15).

\subsubsection{SMT Repository Footprint}

The modeled repository footprint for the HTOM covers an area of $4.65 \mathrm{~km}^{2}$ with a linear power density of $1.35 \mathrm{~kW} / \mathrm{m}$. The modeled repository footprint for the LTOM covers an area of 5.58 $\mathrm{km}^{2}$ with a linear power density of $1.13 \mathrm{~kW} / \mathrm{m}$. The repository footprint corresponds to the area that is heated by the smeared-heat-source representation of heat generation from waste packages. The areal distribution of grid blocks in the repository area of the SMT submodel is the same as that shown in Figure 21, except that the northernmost and southernmost rows of repository subdomains are further subdivided in the SMT submodel to better represent the influence of edge cooling along the northern and southern edges of the repository. 


\subsubsection{SMT-Submodel Mesh}

The SMT-submodel mesh is constructed so that the lateral and lower boundary conditions have a negligible effect on the predicted temperatures near the repository. This is accomplished by placing the lateral boundaries sufficiently far away $(\sim 1000 \mathrm{~m})$ from the repository edges and placing the lower boundary $1000 \mathrm{~m}$ below the water table. The SMT mesh files are output from YMESH, Section 4.2.2 (CRWMS M\&O 2000b [DIRS 149862]; tspa01.mesh01-01 for HTOM and tspa01.mesh0I-03 for LTOM). The process of generating the numerical mesh for the SMT submodel is illustrated in Sub-flowchart 3, Figure 7.

\subsubsection{SMT-Submodel Boundary Conditions}

The SMT-submodel domain extends from the ground surface to $1000 \mathrm{~m}$ below the present-day water table. The lateral boundaries, which are adiabatic boundaries, are situated far enough away from the repository so that they do not affect thermal behavior in the repository. The temperature at the lower boundary of the model domain is extrapolated vertically from the temperature gradient at the water table, based on the calculated temperature field from the UZ Site-Scale Model and on the assumption of Section 3.1.7. Temperature at the water table varies with location, so the extrapolated temperature varies with location at the lower model boundary. The temperature at the ground surface (as well as at the water table) is calculated from the UZ SiteScale Model, using an inverse distance cubed method discussed in Section 3.1.4. The data-flow chart for the preparation of boundary conditions is given in Sub-flowchart 9, Figure 13. The density of the upper boundary is $1.185 \mathrm{~kg} / \mathrm{m}^{3}$ (Section 3.1.7). The heat capacity of the upper boundary is $1 \times 10^{3} \mathrm{~J} / \mathrm{Kg}-\mathrm{K}$ (Section 3.1.7). The thermal conductivity of the upper boundary is $0.0254 \mathrm{~W} / \mathrm{m}-\mathrm{K}$ (Section 3.1.7). This data is incorporated into the SMT submodel through inclusion in the file: SDT-1Dds (CRWMS M\&O 2000b [DIRS 149862]).

\subsubsection{SMT-Submodel Heat Generation Rates}

\subsection{HTOM Heat Generation}

The heat generation rate for the SMT submodel is in the form of a heat-generation rate versus time table located in NUFT include files. During preclosure the file used is output from heatgenAge (BSC, 2001c [DIRS 154461], CRWMS M\&O 2000b [DIRS 149862]; SMT0.3 Qheat-50y_vent-01 for HTOM). The heat output represented in this file accounts for removal of $70 \%$ of the total heat due to ventilation. The postclosure heat generation table is also output from heatgenAge, Section 4.2.16 (BSC 2001c [DIRS 154461], CRWMS M\&O 2000b [DIRS 149862]; SMT-0.3Qheat-1e6y_vent-01).

\subsection{LTOM Heat Generation}

The heat generation rate for the SMT submodel is in the form of a heat-generation rate versus time table located in NUFT include files. During preclosure the file used is output from heatgenAge (BSC 2001c DIRS [154461], CRWMS M\&O 2000b [DIRS 149862]; SMT0.2 Qheat $-300 y_{-}$vent -03 ). The heat output represented in this file accounts for removal of $80 \%$ of the total heat due to ventilation. The postclosure heat-generation table is also output from 
HeatgenAge, Section 4.2.16 (BSC 2001c DIRS [154461], CRWMS M\&O 2000b [DIRS 149862]; SMT-0.2Qheat-1e6y_vent-03).

\subsubsection{SMT-Submodel Material Properties}

The SMT submodel uses thermal-conduction properties (for the stratigraphic units) consistent with the SDT and DDT submodels. These properties are based on Section 3.2.4 through incorporation in the include file SDT-1Dds (CRWMS M\&O 2000b [DIRS 149862]) as illustrated in Sub-flowchart 12, Figure 16. This file incorporates the assumption of Section 3.2.4 by using the wet thermal conductivity. Where saturated zone (SZ) thermal properties are required, the wet thermal conductivity of model unit pp1 is used (Section 3.2.5).

\subsubsection{SMT-Submodel Simulations}

The execution of the SMT submodel is illustrated in Sub-flowchart 16 of Figure 20. Execution varies slightly for the HTOM and LTOM simulations.

\subsection{HTOM Simulations}

Two SMT-submodel HTOM simulations were conducted for this Calculation Report. The first simulation, which is required to calculate pre-closure TH conditions in the MSTH model, had a $70 \%$-reduced heat-generation rate (representing the heat lost to ventilation). However, only the first 50 years of this simulation is used since the ventilation period ends at 50 years. The second simulation, which is required to calculate post-closure TH conditions in the MSTH model, had a simulation period of $1,000,000 \mathrm{yr}$ using a step-function heat-generation rate, with the rate reduced by $70 \%$ for the first $50 \mathrm{yr}$ and the full nominal rate from $50 \mathrm{yr}$ to $1,000,000 \mathrm{yr}$. The two HTOM SMT simulations are applicable to all three infiltration-flux (mean, high, and low flux) scenarios.

\subsection{LTOM Simulations}

Two SMT-submodel LTOM simulations were conducted for this Calculation Report. The first simulation, which is required to calculate pre-closure TH conditions in the MSTH model, had a 80\%-reduced heat-generation rate (representing the heat lost to ventilation). However, only the first 300 years of this simulation is used since the ventilation period ends at 300 years. The second simulation, which is required to calculate post-closure TH conditions in the MSTH model, had a simulation period of $1,000,000$ yr using a step-function heat-generation rate, with the rate reduced by $80 \%$ for the first $300 \mathrm{yr}$ and the full nominal rate from 300 yr to $1,000,000$ yr. The two LTOM SMT simulations are applicable to all three infiltration-flux (mean, high, and low flux) scenarios.

\subsubsection{LDTH SUBMODELS}

The USNT module of NUFT is used to model flow through a fractured porous media in the 2-D LDTH submodels. The key NUFT options used for LDTH simulations include the dualpermeability method (DKM), modified with the active-fracture concept (AFC), to represent twophase heat and fluid flow in the fractured porous rock. These modeling methods are NUFT 
options specified in the NUFT input files. The DKM conceptualizes the fractured rock as having two interacting materials, one representing the matrix and one representing the fractures. The interaction between the fractures and the matrix is explicitly calculated from the local temperature and pressure differences, thus allowing transient behavior to be predicted. The DKM underestimates the fracture-matrix interaction for steep temperature and pressure gradients (Hardin 1998, p. 2 [DIRS 100350]). The AFC accounts for the contact area between the wetted portion of the fracture and the matrix, as well as for the frequency of fractures. The AFC assumes that fracture flow only occurs through a portion of the fractures. This is more conservative than assuming the influx flows ubiquitously through the entire fracture continuum. The flux through a fracture is greater when it has higher saturation and, therefore, focusing flow through a portion of the fractures (i.e., to active fractures) maximizes flux and results in fast pathways for flux through the mountain. The rock properties were calibrated using an inverse modeling technique (LB990861233129.001, LB990861233129.002, LB990861233129.003) The calibrated property sets assume that both the DKM and AFC are used. Consequently, the DKM and AFC NUFT options must be used in the LDTH-submodel calculations.

The LDTH submodels are run at the 33 drift-scale-submodel locations (Figure 22) and for 4 different values of Areal Mass Loading: $A M L=14,27,55$, and 66 MTU/acre for the HTOM and $\mathrm{AML}=11,23,46$, and $55 \mathrm{MTU} / \mathrm{acre}$ for the LTOM. Representing the influence of edgecooling effects requires that most of the LDTH submodel runs use an AML that is less than the nominal value.

\subsubsection{LDTH-Submodel Locations}

The repository footprint is output from the Matlab macro StudyDomain in the file shape.dat (CRWMS M\&O 2000b). The manual operation E:M3 in Sub-flowchart 2, Figure 6 is used to determine the coordinates of the 33 representative drift-scale submodel locations, which are located in the file column.data. These submodel locations, which are used for LDTH and SDT calculations, are shown in Figure 22. There are 64 emplacement drifts within the LTOM repository area. The drift-scale-submodel locations were chosen along eight of those emplacement drifts, including drift nos. 4, 12, 20, 28, 36, 44, 52, and 60 (BSC 2001g [DIRS 154554], BSC 2001h [DIRS 154477]). Drift-scale-submodel locations were uniformly spaced along those eight drifts as shown in Figure 22. These 33 locations were chosen to represent repository-scale variability of thermal properties, hydrologic properties, infiltration flux, and overburden thickness.

\subsection{Stratigraphic Columns}

The stratigraphic columns corresponding to the LDTH-submodel locations are output from YMESH in the file <column>.nft (CRWMS M\&O 2000b [DIRS 149862]). The software used in manipulating the source data into a YMESH input file (excluding the location file, column.data) are rme6 v.1.0 and makeColumn_2001 v1.0. The thickness of the stratigraphic units at each location is output from readUnits (CRWMS M\&O 2000b [DIRS 149862], 33 files: $<$ column.col.units $>$ ), and is tabulated in Table 11 .

\subsection{Vertical Location of Repository Horizon}


The EBS is added to the stratigraphic columns (CRWMS M\&O 2000b [DIRS 149862], <column>.nft) by the routine Define_EBS_fineGrid v1.3, Section 4.2.4. This routine assigns the grid blocks at the location of the EBS (e.g., drift cavity, drip-shield monolith, and invert) to the appropriate material. The routine also changes the vertical resolution of the mesh within the EBS. The vertical location of the repository horizon at the LDTH-submodel locations in the stratigraphic columns <column>.nft, are tabulated by readUnits (CRWMS M\&O 2000b [DIRS 149862], <column>.col.units) and shown in Table 12.

\subsubsection{LDTH-Submodel Mesh}

The numerical mesh for the LDTH submodel is illustrated in Figure 23. This lumped approximation of the drip shield and WP (called the drip-shield monolith) is corrected by the manner in which the pre-closure DDT submodel (Figure 24 for pre-closure, Figure 25 for postclosure), which rigorously accounts for the actual dimensions of the WP (without the presence of the drip shield), is applied in the MSTHAC methodology (Section 2.3).

\subsubsection{LDTH-Submodel Boundary Conditions}

Because the LDTH submodels pertain to a symmetry cell between the vertical plane down the center of the drift and the vertical midplane between drifts, the lateral boundaries are adiabatic and no-mass-flow boundaries. The LDTH submodels require temperature, pressure, and gasphase air-mass fraction at the upper boundary, which represents the ground surface and the lower boundary, which represents the water table (Table 13). Both boundaries are assumed to have constant conditions with time. The density of the upper boundary is $1 \times 10^{8} \mathrm{Kg} / \mathrm{m}^{3}$ (Section 3.1.6). The heat capacity of the upper boundary is $1 \times 10^{3} \mathrm{~J} / \mathrm{kg}-\mathrm{K}$ (Section 3.1.6). The thermal conductivity of the upper boundary is $0.0254 \mathrm{~W} / \mathrm{m}-\mathrm{K}$ (Section 3.1.6). These values are incorporated in the files $d k m$-afc-1Dds-mc-li-01, dkm-afc-1Dds-mc-mi-01, and $d k m-a f c-1 D d s-$ mc-ui-01 (CRWMS M\&O 2000b [DIRS 149862]).

An interpolation routine is used to relate the temperatures and pressures at various points to the LDTH-submodel locations. The routines used for this interpolation are chim_surf_wt_TP2 (Section 4.2.9). The air mass fraction and liquid enthalpy are found given the resulting temperature and pressure, along with a bounding assumption of $100 \%$ humidity (Section 3.1 .5 ). The routine used for this calculation is chim_surf_wt_TP2 v.1.0 (Section 4.2.9).

\subsubsection{LDTH-Submodel Heat-Generation Rates}

The heat generation rates for the LDTH submodels are in the form of heat-generation rate versus time tables located in NUFT include files (BSC 2001g [DIRS 154554], BSC 2001h [DIRS 154477]). During preclosure the file used is output from HeatgenAge v.1.2, Section 4.2.16 (CRWMS M\&O 2000b [DIRS 149862]; LDTH-SDT-0.3Qheat-50y_vent-0I for HTOM and LDTH-SDT-0.2Qheat-300y_vent-03 for LTOM). The heat-generation rate given in this file accounts for removal of $70 \%$ for HTOM and $80 \%$ for LTOM of the total heat due to ventilation. The postclosure heat-generation table is also output from HeatgenAge v.1.2, Section 4.2.16 (CRWMS M\&O 2000b [DIRS 149862]; LDTH-SDT-0.3Qheat-1e6y_vent-01 for HTOM and LDTH-SDT-0.2Qheat-1e6y_vent-03 for LTOM). 
Because any given LDTH submodel covers the same model domain (including the same area in plan view) as the corresponding SDT submodel, the LDTH submodel and corresponding SDT submodel use the same heat-generation rate versus time tables (files).

\subsubsection{LDTH-Submodel Material Properties}

Material properties are output from the macros Rock_LDTH v1.0 (Section 4.2.11) into three files (CRWMS M\&O 2000b, dkm-afc-1Dds-mc-mi-01, dkm-afc-1Dds-mc-li-01, dkm-afc-1Dds-mc-ui01), each one corresponding to an infiltration rate. Properties corresponding to the base case infiltration flux are given in the files 1d-driftscale_basecase2_flow.prn and Id_driftscale_basecase2_th.prn (LB990861233129.001). Properties corresponding to the upper infiltration flux are given in the files ld-driftscale_lowinfflow.prn and Id_driftscale_lowinf_th.prn (LB990861233129.002). Properties corresponding to the lower bound infiltration flux are given in the files 1d-driftscale_upperinf_low.prn and 1d_driftscale_upperinf_th.prn (LB990861233129.003). These files are renamed and read into the routine Rock_LDTH (Section 4.2.11, see Sub-flowchart 11, Figure 15). The LDTH EBS properties are based on inputs in Section 5.2 and assumptions in Section 3.2.

\subsubsection{Infiltration Flux}

Infiltration-flux data is given in the nine files from Section 4.2.19, representing three cases (low, mean, and upper, each having three climates (present day, monsoon, and glacial). Infiltration rates at each submodel location in column.data (CRWMS M\&O 2000b [DIRS 149862]) are found using a routine, ColumnInfiltration v1.1 (Section 4.2.8), that interpolates and then normalizes the results from the 33 drift-scale submodel locations.

CONVERTCOORDS v.1.1 (Section 4.2.1) prepares the input data for the interpolation routine, ColumnInfiltration v1.1 (see Sub-flowchart 8, Figure 12). The output from ColumnInfiltration is one large file (CRWMS M\&O 2000b [DIRS 149862]; infiltration.tex). This file is split into the nine constituent infiltration rates with the routine infiltab. The average of the 33 infiltration rates was found to differ from the average of all the infiltration rates in the source data that were within the repository footprint. To account for this the infiltration rates are normalized with respect to the average of the source data over the repository footprint.

\subsubsection{LDTH-Submodel Simulations}

Because of the manner in which the MSTH model temperatures are abstracted, it is necessary to account for drift ventilation scenarios by conducting two complete (parallel) MSTH model abstractions: (1) a pre-closure simulation and (2) a post-closure simulation. The results of these two MSTH model abstractions are spliced at the end of the pre-closure period, i.e., when ventilation ceases. Therefore, it is necessary to run two complete sets of MSTH submodels to account for ventilation for each infiltration-flux case. The LDTH submodel is the only submodel type that has to be run for each of the three infiltration-flux cases (mean, high, and low flux). The simulations for the other three submodel types are applied to all three cases. The execution procedure of the LDTH submodel is illustrated in Sub-flowchart 13, Figure 17. 
For a given infiltration-flux case, two sets of LDTH-submodel simulations are required. The first set, which is required to calculate pre-closure TH conditions in the MSTH model, was run with the heat-generation rate reduced by $70 \%$ for 50 years in the case of HTOM and with the heatgeneration rate reduced by $80 \%$ for 300 years in the case of LTOM. The second set, which is required to calculate post-closure TH conditions in the MSTH model, was run for a simulation period of $1,000,000 \mathrm{yr}$ starting on the final conditions of the pre-closure runs. For the HTOM simulation the model is started at $\mathbf{5 0}$ years and run at the full nominal heat-generation rate to $1,000,000$ years; for LTOM the model is started at 300 years and run at the full nominal heat rate to $1,000,000$ years.

Each LDTH-submodel set consists of 132 simulations, the result of 4 AML simulations at each of the 33 drift-scale-submodel locations. Thus, because there are two sets (pre- and post-closure), a total of 264 LDTH simulations are conducted for any given infiltration-flux case. Finally, because there are three infiltration-flux cases investigated, a total of 792 LDTH simulations are executed for each operating mode (HTOM or LTOM) in this Calculation Report.

\subsubsection{SDT SUBMODELS}

The 1-D SDT submodels are run in parallel with the LDTH submodels at the same 33 locations and for the same AMLs: 14, 27, 55, and 66 MTU/acre for HTOM and 11, 23, 46, and 55 MTU/acre for LTOM. These submodels are required to obtain functional relationships between "line-averaged" temperatures predicted by the LDTH submodel and the "smeared" host-rock temperatures predicted by the SDT submodel.

\subsubsection{SDT-Submodel Mesh}

The SDT submodels use the same vertical discretization of grid blocks as is used in the SMT submodels. The multi-scale modeling methodology demands consistency between how heat flow is modeled in the respective SDT and SMT submodels, including consistency in the vertical gridblock discretization in the respective submodels. The procedure for generation the submodel mesh is given in the Sub-flowchart 3 of Figure 7.

\subsubsection{SDT-Submodel Boundary Conditions}

The data-flow chart for the preparation of boundary conditions is given in Sub-flowchart 9, Figure 13. Because the SDT submodels are for a symmetry cell between the vertical plane down the center of the drift and the vertical midplane between drifts, the lateral boundaries are adiabatic and no-mass-flow boundaries. The SDT submodels require temperature at the upper boundary, which represents the ground surface and the lower boundary, which represents the water table (Table 13). Both boundaries are prescribed constant temperature conditions with time. The density of the upper boundary is $1.185 \mathrm{Kg} / \mathrm{m}^{3}$ (Section 3.1 .7 ). The heat capacity of the upper boundary is $1 \times 10^{3} \mathrm{~J} / \mathrm{Kg}-\mathrm{K}$ (Section 3.1.7). The thermal conductivity of the upper boundary is $0.0254 \mathrm{~W} / \mathrm{m}-\mathrm{K}$ (Section 3.1.7). This data is incorporated into the SDT submodel through inclusion of the file: SDT-1Dds (CRWMS M\&O 2000b [DIRS 149862]).

The temperature and pressure are based on the grid in Section 5.2.11, the temperatures and pressures in Section 5.2.14, and the locations in column.data (CRWMS M\&O 2000b [DIRS 
149862]). An interpolation routine is used to relate the temperatures and pressures at various points to the SDT-submodel locations. The routine used for this interpolation is chim_wt_TP2 (Section 4.2.9). The air mass fraction and liquid enthalpy is found given the resulting temperature and pressure, along with a bounding assumption of $100 \%$ humidity (Section 3.1.5). The routine used for this calculation is chim_surf_wt_TP2 (Section 4.2.9).

\subsubsection{SDT-Submodel Heat Generation Rates}

Because any given SDT submodel covers the same model domain (including the same area in plan view) as the corresponding LDTH submodel, the SDT submodel and corresponding LDTH submodel use the same heat-generation rate versus time tables. These are NUFT include files output from HeatgenAge v.1.2, Section 4.2.16 (CRWMS M\&O 2000b [DIRS 149862]; LDTHSDT-0.3Qheat-50y_vent-01 for HTOM and LDTH-SDT-0.2Qheat-300y_vent-03 for LTOM).

\subsubsection{SDT-Submodel Material Properties}

The SDT submodel uses the same thermal-conduction properties (for the stratigraphic units) that are used in the SMT submodel. These properties are based on Section 3.2.4 through incorporation in the include file $S D T-1 D d s$ (CRWMS M\&O 2000b [DIRS 149862]) as illustrated in Sub-flowchart 12, Figure 16.

\subsubsection{SDT-Submodel Simulations}

Each SDT-submodel set consists of 132 simulations, the result of 4 AML simulations at 33 driftscale-submodel locations. Each SDT simulation is valid for all three infiltration-flux (mean, high, and low flux) cases. As for the LDTH submodel, two sets of simulation were run. The first pre-closure set was run with the heat-generation rate reduced by $70 \%$ for 50 years and with the heat-generation rate reduced by $80 \%$ for 300 years. The second post-closure set was run starting from final pre-closure run results at 50 years for HTOM (300 years for LTOM) to $1,000,000 \mathrm{yr}$ using the full nominal heat-generation rate. Thus, a total of 264 SDT simulations were conducted for each operating mode case (HTOM and LTOM). The execution procedure of the SMT submodel is illustrated in Sub-flowchart 16, Figure 20.

\subsubsection{DDT SUBMODELS}

The 3-D DDT submodel is used to determine the WP-specific temperature deviations relative to line-averaged-heat-source conditions in the NFE and the EBS (e.g., drift wall, drip shield, and WP). The DDT accounts for WP-specific heat output and for thermal radiation between all WP and drift surfaces. For the pre-closure period prior to the emplacement of the drip shield and for the post-closure period, thermal radiation between the WP and drift surfaces controls the longitudinal temperature deviations along the drift. The values of thermal conductivity in the host rock play a minor role on the magnitude of longitudinal temperature deviations along the drift (Hardin 1998, Section 3.7.5.4 [DIRS 100350]).

The DDT submodel is used for two purposes: (1) calculating the temperature difference between the WP and drip shield and (2) calculating the longitudinal temperature variations along the drift axis. As neither of these quantities is significantly influenced by the thermal conductivity in the 
host rock or in any of the other stratigraphic units, it is not necessary to run the DDT submodels at multiple locations. Further, advective heat transfer driven by TH behavior in the host rock has little effect on axial temperature variation in the drift, such that TH processes in the host rock do not contribute significantly to equalization of axial temperature variations in the drift. The conduction/radiation-only DDT model adequately represents longitudinal deviations in NFE and EBS temperatures (relative to line-average-heat-source conditions) along the drift. Hence, the DDT submodel is run at one drift-scale-submodel location (15c3 in Figure 22).

\subsubsection{DDT-Submodel Location}

The 15c3 drift-scale-submodel location (CRWMS M\&O 2000b [DIRS 149862]; column.data) is used for all DDT submodels.

\subsubsection{DDT-Submodel Mesh}

The cross-sectional dimensions of the drift for the post-closure period is shown in Figure 25; these dimensions were used to build the numerical meshes of the DDT submodels. For the pre-closure period, the drip shield in Figure 24 is not present; otherwise all other dimensions are the same as in the post-closure case. All of the DDT-submodel meshes assume circular cross section for the drift (just as in the LDTH submodel) and a square cross section for the WP and drip shield (where it is present). The cross-sectional area of the "square" WP in the DDT submodel is the same as that of a 1.67-m-diameter WP. As shown by Wilder (1996, Table 1.10.4.1 [DIRS 100792]), a circular WP in a circular drift can be accurately represented by a square WP in a square drift, provided that the respective circular and square cross-sectional areas are equal.

The longitudinal dimensions of the drift are shown in Figure 26 for HTOM and Figure 27 for LTOM. The DDT submodel utilizes symmetry in all four directions: about (1) the vertical midplane down the center of the drift, (2) the vertical midplane down the center of the rock pillar between drifts, (3) the vertical plane that is orthogonal to and intersects the "pwr1-1" WP, and (4) the vertical plane that is orthogonal to and intersects the "bwr1-3" WP.

The DDT-submodel mesh uses fine grid-block spacing in the lateral and vertical direction in the drift to account for the drip-shield cross section, the gap between the WP and drip shield, and (3) thermal conduction in the invert. The generation of the numerical meshes for the DDT model is illustrated in the Sub-flowchart 1 of Figure 5.

\subsubsection{DD-Submodel Boundary Conditions}

The boundary conditions for the DDT submodels are the same as those for the SDT submodel at the $15 \mathrm{c} 3$ drift-scale-submodel location (see Figure 22). Similarly the DDT boundary conditions are the same as the corresponding LDTH submodel.

Because the DDT submodels are for a symmetry cell between the vertical plane down the center of the drift and the vertical midplane between drifts, the lateral boundaries are adiabatic and nomass-flow boundaries. The DDT submodels require temperature at the upper boundary, which 
represents the ground surface and the lower boundary, which represents the water table (Table 13). Both boundaries have constant temperature conditions with time.

The density of the upper boundary is $1.185 \mathrm{~kg} / \mathrm{m}^{3}$ (3.1.7). The heat capacity of the upper boundary is $1 \times 10^{3} \mathrm{~J} / \mathrm{Kg}-\mathrm{K}$ (3.1.7). The thermal conductivity of the upper boundary is 0.0254 $\mathrm{W} / \mathrm{m}-\mathrm{K}$ (3.1.7). This data is incorporated into the DDT submodel through inclusion in the file: SDT-1Dds (CRWMS M\&O 2000b [DIRS 149862]).

The temperature and pressure are based on the grid in Section 5.2.11, the temperatures and pressures in Section 5.2.14, and the locations in column.data (CRWMS M\&O 2000b [DIRS 149862]). An interpolation routine is used to relate the temperatures and pressures at various points to the DDT-submodel locations. The routines used for this interpolation are chim_surf_wt_TP2, Section 4.2.9.

\subsubsection{DDT-Submodel Heat Generation Rates}

The preparation of heat-generation tables for the DDT submodel is illustrated in the Subflowchart 7 of Figure 11. Heat-generation-rate versus time tables, taken from the source data described in Section 5.2.13, are required for the 10 different waste packages represented in the DDT submodels (Figure 26 for HTOM and Figure 27 for LTOM). The heat-generation rate for the DDT submodel is in the form of a heat-generation rate versus time table located in NUFT include files. During preclosure the file used is output from HeatgenAge v.1.2, Section 4.2.16 (CRWMS M\&O 2000b [DIRS 149862]; DDT-0.3Qheat-50y_vent-01 for HTOM, DDT0.2 heat-300y_vent-03 for LTOM). The heat output represented in this file accounts for removal of $70 \%$ for HTOM and $80 \%$ for LTOM of the total heat due to ventilation. The postclosure heat generation table is also output from HeatgenAge v.1.2 (CRWMS M\&O 2000b [DIRS 149862]; SMT-0.3Qheat-1e6y_vent-01 for HTOM, SMT-0.2Qheat-300y_vent-03 for LTOM).

\subsubsection{DDT-Submodel Material Properties}

The DDT submodel uses the same thermal-conduction properties (for the stratigraphic units) that are used in the SMT and SDT submodels. These properties are based on Section 5.2.9 and Section 3.2.4 through incorporation in the include file SDT-1Dds (CRWMS M\&O 2000b [DIRS 149862]) as illustrated in Sub-flowchart 11, Figure 15. This file incorporates assumption 3.2 .4 by using the wet thermal conductivity. In addition to the properties for the stratigraphic units, the DDT submodels require the thermal-conduction properties for the EBS materials (Table 14). For the drip shield, and crushed-tuff invert, the DDT submodels use the same thermal-property values as those used in the LDTH submodels.

The thermal properties of the gap between the WP and drip shield and between the drip shield and the drift surfaces are those of air.

The solid density and specific heat of the air gap in the drift are standard handbook values (Section 5.2.8). Assumption 3.2.7 is used for thermal conductivity for the invert.

For the HTOM, thermal radiation is explicitly represented throughout the drift for all simulation time. For the LTOM, thermal radiation is explicitly throughout the emplacement drift for the first 
$10,000 \mathrm{yr}$. Thereafter, thermal radiation continues to be explicitly represented between the WP and drip shield; however, for the cavity between the drip shield and drift surfaces, thermal radiation is approximated using an effective thermal conductivity versus time relationship (Section 5.2.3). Recall that the DDT submodel is used to predict two quantities: (1) the temperature difference between the WP and drip shield and (2) the longitudinal temperature deviations. The use of an effective thermal conductivity in the cavity between the drip shield and drift surfaces only affects the latter quantity. It should be noted that the longitudinal temperature deviations have greatly diminished by $10,000 \mathrm{yr}$; consequently, the use of this effective thermal conductivity has a negligible effect on the MSTH-model results.

\subsubsection{DDT-Submodel Simulations}

Preclosure and postclosure DDT-submodel simulations were conducted for this Report. These DDT simulations are applicable to allthree infiltration-flux (mean, upper, and lower) cases.

The first, which is required to calculate pre-closure TH conditions in the MSTH model, was run with the heat-generation rate reduced by $70 \%$ for HTOM and $80 \%$ for LTOM. The first DDT submodel has no drip shield in the drift (Figure 24).

The third DDT submodel, which is required to calculate post-closure TH conditions in the MSTH model, was run for a simulation period from 0 yr to $1,000,000 \mathrm{yr}$ using the step-function heat-generation rate with the rate reduced by either 70 or 80 percent during the preclosure period and stepped up to the full nominal rate during the postclosure period. The execution procedure of the DDT submodel is illustrated in Sub-flowchart 15, Figure 19.

\subsection{MSTHAC Submodel (LMTH, DMTH) Abstraction}

The use of MSTHAC to assemble the execution results of the NUFT submodels into final output is the second part of the MSTH model (see Figure 2). MSTHAC assembles the execution results from the submodels creating time-varying AML curves. An intermediate Line-averaged-heatsource Mountain-scale Thermohydrologic (LMTH) model and the final Discrete-source Mountain-scale Thermohydrologic (DMTH) model are then created. The MSTHAC approach can be broken into five steps in centered upon the assembly of two time-varying AMLs: a hostrock effective AML ( $\left.A M L_{\text {effective }}\right)$ and a WP-specific AML $\left(A M L_{\text {specific }}\right)$. The $A M L_{\text {effective }}$ which is based on comparing the results from a 3-D SMT submodel with those of corresponding 1-D SDT submodels, captures the influence of 3-D mountain-scale heat flow. The $A M L_{\text {specific }}$ which is a

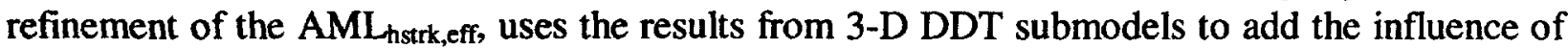
WP-specific deviations in local temperature onto the influence of mountain-scale heat flow. Both AMLs are used to interpret LDTH model results to the LMTH and DMTH models. The five step process of MSTHAC is illustrated in Figure 28 and is explained in detail below.

\subsubsection{MSTHAC Step 1: Assemble Effective Host-Rock Areal Mass Loading, AMLeffective}

The temperature history from the SDT submodel is plotted for each of the 33 spatial locations for a 'family' of four AMLs (66, 55, 27 and 14 MTU/acre for the HTOM; 55, 46, 23 and 11 MTU/acre for the LTOM). The temperature results are then spatially interpolated to the 671 locations for HTOM (762 locations for LTOM). The host-rock temperature $T_{\text {hstrk }}$ calculated by 
the 3-D SMT submodel is compared against those calculated by the family of SDT submodels. The $A M L_{\text {effective }}$ is obtained by interpolating the SMT-calculated $T_{\text {hstrk }}$ among the family of SDTcalculated $T_{\text {hstrk }}$ for each timestep.

\subsubsection{MSTHAC Step 2: Interpolate LMTH-Model Temperatures}

The next step in MSTHAC involves the family of LDTH submodels. For each timestep, the perimeter-averaged drift-wall temperature $T_{\mathrm{dw}}$ is obtained by interpolation, using $A M L_{\text {effective }}$ and the family of four LDTH-calculated $T_{\mathrm{dw}}$ curves (i.e., for each of the four AMLs). Because this value of $T_{\mathrm{dw}}$ is the result of a line-averaged-heat source and because it accounts for 3-D mountain-scale heat flow, it is called $T_{\mathrm{dw}, \mathrm{LMTH}}$. This process of generating LMTH-model temperatures is repeated at other drift-scale locations, such as in the invert and at various locations in the host rock, for all 671 repository locations in the HTOM and for all 762 repository locations in the LTOM.

\subsubsection{MSTHAC Step 3: Calculate DMTH-Model TH Variables}

After LMTH-model temperatures have been determined at all locations of interest, including the drift wall, invert, and various locations in the host rock, the next step in MSTHAC is to incorporate the influence of WP-to-WP variability in heat output, which is obtained from the family of DDT submodels. Local deviations in temperature (relative to line-heat-source-averaged conditions) are determined using a family of four DDT submodels (for each of the four AMLs). These temperature deviations are superimposed onto the LMTH-model temperature results. This process of generating DMTH-model temperatures is repeated at other drift-scale locations, such as in the invert and at various locations in the host rock, for all 671 repository locations in the HTOM and for all 762 repository locations in the LTOM.

\subsubsection{MSTHAC Step 4: Assembling Waste-Package-Specific Areal Mass Loading, $A M L_{\text {specific }}$}

The procedure for assembling $A M L_{\text {specific }}$ is very similar to that of assembling $A M L_{\text {effective. The }}$ DMTH-model temperatures are compared against the family of LDTH-submodel temperatures. The $A M L_{\text {specific }}$ is obtained by interpolating the DMTH-calculated temperature curve among the family of LDTH-calculated temperature curves for each timestep. This process is repeated at other drift-scale locations, such as in the invert and at various locations in the host rock, for all 671 repository locations in the HTOM and for all 762 repository locations in the LTOM.

\subsubsection{MSTHAC Step 5: Interpolate TH Variables for DMTH}

Once $A M L_{\text {specific }}$ is determined at a particular geographic and waste-package-specific location, it is possible to determine corresponding thermohydrologic variables such as relative humidity. For example, the perimeter-averaged drift-wall relative humidity $R H_{\mathrm{dw}}$ is obtained by interpolation, using the $A M L_{\text {specific }}$ and the family of LDTH-calculated $R H_{\mathrm{dw}}$ curves. This process of generating DMTH-model TH variables is repeated at other drift-scale locations, such as in the invert and at various locations in the host rock, for all 671 repository locations in the HTOM and for all 762 repository locations in the LTOM. This process is repeated for all TH variables. 


\subsubsection{MSTHAC Step 6: Determine Relative Humidity on the Drip Shield and Waste Package}

The last MSTHAC calculation step involves the determination of relative humidity $R H$ on the drip shield $R H_{\mathrm{ds}}$ and waste package $R H_{\mathrm{wp}}$; this output also pertains to the DMTH model. The values of $R H_{\mathrm{ds}}$ and $R H_{\mathrm{wp}}$ are obtained in an analogous fashion. During the pre-closure period there is no drip shield over the waste package and $R H_{\mathrm{wp}}$ is obtained from the following expression:

$$
R H_{w p}=R H_{d w} \frac{P_{s a t}\left(T_{d w}\right)}{P_{s a t}\left(T_{w p}\right)},
$$

where $R H_{\mathrm{dw}}$ and $T_{\mathrm{dw}}$ are the perimeter-averaged $R H$ and $T$ on the drift wall, $T_{\mathrm{wp}}$ is the wastepackage temperature and $P_{\text {sat }}$ is the saturated vapor pressure. Note that Eqn. 1 holds in the absence of water dripping onto the waste package. Note also that Eqn. 1 does not account for the presence of relatively dry ventilation air in the drift.

For the post-closure period, the drip shield overlies the waste package and the following expression applies to $R H$ on the drip shield $R H_{\mathrm{ds}}$ :

$$
R H_{d s}=R H_{d w, c a v} \frac{P_{s a t}\left(T_{d w}\right)}{P_{s a t}\left(T_{d s}\right)}
$$

where $R H_{\mathrm{dw}}$ and $T_{\mathrm{dw}}$ are the perimeter-averaged $R H$ and $T$ on the drift wall, $T_{\mathrm{ds}}$ is the perimeteraveraged $T$ on the drip shield, and $P_{\text {sat }}$ is the saturated vapor pressure. Note that Eqn. 2 holds in the absence of water dripping onto the drip shield. Depending on the magnitude of this dripping flux, $R H$ reduction on the drip shield will be diminished (CRWMS, 2000b).

For the post-closure period, the drip shield overlies the waste package and the following expression applies to $R H$ on the waste package $R H_{\mathrm{wp}}$ :

$$
R H_{w p}=R H_{d s} \frac{P_{s a t}\left(T_{d s}\right)}{P_{s a t}\left(T_{w p}\right)},
$$

where $R H_{\mathrm{ds}}$ and $T_{\mathrm{ds}}$ are the perimeter-averaged $R H$ and $T$ on the drip shield, $T_{\mathrm{wp}}$ is the wastepackage $T$, and $P_{\text {sat }}$ is the saturated vapor pressure. Note that Eqn. 3 holds in the absence of water dripping onto the waste package.

\subsection{MSTH-Model Cases}

The MSTH model is run for each case scenario, i.e., for each simulated repository scenario including pre-closure and post-closure. NUFT submodels are executed according to Section 2.2. Submodel results are extracted and integrated using MSTHAC according to Section 2.3. The final results of this process are the results of a MSTH-model simulation case. Each MSTHmodel simulation case represents a complete pre- and post-closure repository simulation for a 
specific scenario (e.g., the mean-infiltration-flux scenario) and for a specific repository operating-mode case (e.g., LTOM with line-load WP spacing and wider drift spacing).

\subsubsection{Key Factors Accounted for by the MSTH Model}

The MSTH model captures the influence of key factors affecting TH conditions in the emplacement drifts and in the surrounding host rock:

- Repository-scale variability of the local percolation flux

- Temporal variability of percolation flux (as influenced by climate change)

- Uncertainty in percolation flux (as represented by the mean, high, and low infiltration flux scenarios)

- Repository-scale variability in hydrologic properties (e.g., those properties which control fracture-matrix interaction and capillarity in fractures and matrix)

- Edge-cooling effect, which increases with proximity to the edges of the repository

- Dimensions and properties of the EBS components, such as the drip shield and invert

- Waste package-to-waste package variability in heat-generation rate

- Repository-scale variability in overburden thickness

- Repository-scale variability in rock thermal conductivity with an emphasis on the host-rock units.

\subsubsection{Total Submodel Simulations Per MSTH-Model Case}

The MSTH model involves mountain-scale calculations and drift-scale calculations at 33 repository locations to capture the influence of the repository/EBS design and operational parameters as well as the variability of TH properties, overburden thickness, boundary conditions, and percolation flux. For this Calculation Report a given MSTH-model case requires a large number of submodel calculations including the following: (1) one SMT-submodel simulation, (2) 4 DDT-submodel simulations, (3) 396 (33 locations $\times 4$ AMLs x 3 infiltrationflux scenarios) LDTH-submodel simulations, and (4) 396 (33 locations x 4 AMLs x 3 infiltration-flux scenarios) SDT-submodel simulations. To model the preclosure ventilation period, the number of model runs was doubled (one pertains to the pre-closure period and the other pertains to the post-closure period), but these additional runs were for a shorter time period (CRWMS M\&O 2000b [DIRS 149862], Section 6.6.1). Ventilation was modeled using a WPdesign criteria that 70 percent of the heat generation removed (by ventilation) for $50 \mathrm{yr}$ for the HTOM and 80 percent be removed for $300 \mathrm{yr}$ for the LTOM, as stated in the Ventilation Model (CRWMS M\&O 2000k [DIRS 120903], Section 6.1). 
Temperature, relative humidity, and other quantities were calculated for 671 repository locations for the HTOM and for 762 for the LTOM. These quantities were abstracted by Francis (2001??) for the two principal WP groups (CSNF and HLW WPs). The 671 HTOM locations are shown in Figure 21.

Ten different waste packages are considered at each of the 671 locations for the HTOM (762 for the LTOM), resulting in 6710 individual environment histories for the HTOM (7620 for the LTOM). These histories were abstracted into 30 groups for WP corrosion, radionuclide release, and EBS-transport calculations. The same TH results were determined for all three infiltration-flux scenarios (mean-, high-, and low-flux scenarios) in the TSPA-SR model. The TH results were combined into ten distinct groups: five infiltration-flux bins (grouped on the basis of infiltration flux) for each of the two WP groups (CSNF and HLW WPs) (CRWMS M\&O 2001 [DIRS 154594). However, because of the importance of the variability in waste package failure time, the full suite of 1342 sets of HTOM results and 1524 LTOM results (number of repository locations times two WP groups) were provided as input to the WP-and drip-shield-degradation models. Where bin averaging was not noted in TSPA-SR (CRWMS M\&O 2000j [DIRS 153246], Figure 3.3-7), the full set of spatial locations is identified. A complete list of EBS and NFE variables calculated with the MSTH model at all locations is provided in Table 14.

The procedures used for averaging the various $\mathrm{TH}$ quantities over the infiltration bins are described in detail in Abstraction of NFE Drift Thermodynamic Environment and Percolation Flux (CRWMS M\&O 2001 [DIRS 154594]). There is one case where the thermal hydrology abstraction does not simply average information from the MSTH model which is in the determination of the evaporation rate of water at the top of the drip shield. One of the parameters used for the in-drift chemical environment abstraction is the ratio of water evaporation rate to water in flow rate. The MSTH model can calculate these quantities only in a porous medium, which is not the situation at the top of the drip shield. The seepage flow rate at the top of the drip shield is taken from the seepage abstraction, and the evaporation rate is bounded by the amount of heat available to vaporize water on the upper portion of the drip shield. These bounding values are used to estimate how much water could be evaporated by the waste package heat output at any given time (CRWMS M\&O 2001 [DIRS 154594], Section 6.3.10).

\subsubsection{Accounting for the Influence of Drift Ventilation}

For an MSTH model scenario that does not involve drift ventilation, the calculation sequence described in the following sections is executed once. For an MSTH-model scenario that involves drift ventilation during the pre-closure period (which is the case for this Calculation Report), the following MSTH model calculation sequence is executed twice, once for pre-closure and once for post-closure. The sequence of running pre-closure and post-closure runs for each of the submodels is discussed in their respective subsections of Section 2.2.

\subsubsection{Accounting for the Emplacement of the Drip Shield}

If an MSTH-model case involves the emplacement of a drip shield, it is necessary to break up the LDTH- and DDT-submodel calculations into two parts. For the pre-closure period, the LDTH 
and DDT submodels are conducted with a drift without the drip shield. A restart file is generated for each of the pre-closure submodel runs to start the corresponding post-closure submodel run that includes either a drip shield; the post-closure submodel is started at the time of closure.

\subsubsection{Binning TH Results}

For each of the repository location (also referred to as a repository subdomain) the WP sequencing as represented in the DDT model is as follows: 1/2 PWR, DHLW-L, PWR, BWR, DHLW-L, BWR, DHLW-S, PWR, and $1 / 2$ BWR. The WP spacings are shown in Figure 26 for the HTOM and Figure 27 for the LTOM.

For a given repository subdomain and WP group, an average WP temperature history and an average WP relative humidity history are calculated on the basis of a simple average. For each WP in the group, the sum of the normalized differences is summed over time as follows:

$$
\sum_{t=0}^{t=1,000,000} \frac{\left(T_{\mathrm{WP}}-T_{\mathrm{WP}, \text { avg }}\right)}{\left(T_{\mathrm{WP}, \mathrm{avg}}\right)^{2}}+\frac{\left(R H_{\mathrm{WP}}-R H_{\mathrm{WP}, \text { avg }}\right)}{\left(T_{\mathrm{WP}, \mathrm{avg}}\right)^{2}}
$$

where $T_{\mathrm{WP}}$ is the WP temperature (calculated in Section Error! Reference source not found.), $R H_{\mathrm{WP}}$ is the WP relative humidity, $T_{\mathrm{WP}, \text { avg }}$ is the average WP temperature (based on a simple average) and $R H_{\mathrm{WPavg}}$ is the average WP relative humidity (based on a simple average) in that repository subdomain and WP group. The WP with the minimum sum is selected as being represented of that repository subdomain and WP group.

\subsection{TH Variable Calculations in MSTH Model}

Temperatures are the first variables to be calculated in the MSTH model. After LMTH-model temperatures have been determined at all locations of interest, including the drift wall, invert, and various locations in the host rock, the next step in MSTHAC is to incorporate the influence of waste-package-to-waste-package variability in heat output, which is obtained from the family of DDT submodels. The results of this step are the DMTH-model temperatures. The specific variables solved for with the MSTH model are listed in Table 14.

\section{ASSUMPTIONS}

\subsection{Boundary Conditions}

\subsubsection{SMT Submodels}

The heated footprint of the repository area is adequately represented as shown in Figure 21 for the HTOM and LTOM. The variability of surface topography and temperatures across the top boundary of the repository is adequately represented by the gridding of the SMT submodels.

Rationale: The actual repository footprint has an area of $4.60 \mathrm{~km}^{2}$ for the HTOM and $5.52 \mathrm{~km}^{2}$ for the LTOM (BSC 2001g [DIRS 154554], BSC 2001h [154477]). The modeled repository footprint is $4.65 \mathrm{~km}^{2}$ for the HTOM and $5.58 \mathrm{~km}^{2}$ for the LTOM, which are both within 1 
percent of the actual areas of the LTOM and the HTOM. The SMT-submodel grid resolution (and corresponding MSTH-model grid resolution) is precise enough that the MSTH-model results would not be affected by increasing the grid resolution. This assumption does not require confirmation.

\subsubsection{Drift-scale-Submodel Locations}

The 33 drift-scale-submodel locations are evenly spaced throughout the repository as shown in Figure 22. The variability of stratigraphy, temperature, pressure, and infiltration flux across the top boundary of the repository is adequately represented by determining these values at the 33 locations in column.data (CRWMS M\&O 2000b [DIRS 149862]) and illustrated in these figures. This assumption is implicit in the file column.data (CRWMS M\&O 2000b [DIRS 149862]).

Rationale: The 33 drift-scale-submodel locations are evenly spaced throughout the repository area and capture the repository-scale variability of the key variables such as overburden thickness, local host-rock unit, and infiltration flux. The MSTH-model results would not be significantly affected by increasing the number of drift-scale-submodel locations. This assumption does not require confirmation.

\subsubsection{Gaussian Interpolation}

The normalized results from Gaussian interpolation [(Isaaks and Srivastava 1989 [DIRS 109018], p. 208) and (Kitanidis 1997 [DIRS 101403], p. 54); see Eq. XIV-2] adequately represent infiltration rates.

This assumption is used in all LDTH submodels through incorporation in the NUFT input files (CRWMS M\&O 2000b [DIRS 149862]; files: *.in). The interpolated data is in the files output from ColumnInfiltration (CRWMS M\&O 2000b [DIRS 149862]; 9 files: *.out; see Subflowchart 8, Figure 12).

Rationale: The Gaussian method strongly weights the closest points. For a given point, the infiltration rates at relatively close points are the best indicators. It was the author's choice to use this method for its application in this document. This assumption does not require confirmation.

\subsubsection{Inverse Distance Interpolation}

The inverse distance equation (Isaaks and Srivastava 1989 [DIRS 109018], p. 258. See Table XIII-1 for this equation) is appropriate for interpolating temperature and pressures at model boundaries. Chim_surf_wt_TP2 (Section 4.2.9) interpolate the temperature and pressure at the ground surface and water table using the inverse distance cubed equation. SMT_surf_bc3 and SMT_bot_bc3 (Sections 4.2.13 and 4.2.14) use the inverse distance squared equation to interpolate temperatures at the boundaries of the SMT submodels (Section 2.2.1.3).

Rationale: The inverse distance method strongly weights the closest points. The actual power chosen is subjective and has a negligible effect on the numerical results. For a given point, the temperature and pressure at relatively close points are the best indicators. It was author's choice to use this method for its application in this document. This assumption does not require confirmation. 


\subsubsection{Relative Humidity at the Ground Surface}

The relative humidity at the ground surface is assumed to be $100 \%$. This assumption affects all LDTH-submodel input files.

Rationale: The infiltration flux used in the LDTH submodels is the net infiltration flux that already accounts for the influence of evapotranspiration in the soil zone at Yucca Mountain (CRWMS M\&O 2000 [DIRS 143244]. Thus, the influence of the relatively low atmospheric relative humidity at Yucca Mountain has already been implicitly factored into the LDTH submodels. It would be invalid to apply the actual atmospheric relative humidity conditions at Yucca Mountain in the LDTH submodels because this would result in the double-accounting of the influence of evaporation on infiltration flux. This assumption does not require confirmation.

\subsubsection{LDTH-Submodel Boundary Conditions}

The density of the upper boundary is assumed to have a value of $1 \times 10^{8} \mathrm{Kg} / \mathrm{m}^{3}$. The heat capacity of the upper boundary is assumed to have a value of $1 \times 10^{3} \mathrm{~J} / \mathrm{Kg}-\mathrm{K}$. The thermal conductivity of the upper boundary is assumed to have a value of $0.0254 \mathrm{~W} / \mathrm{m}-\mathrm{K}$.

Rationale: The values used for this boundary condition are required in the NUFT input files, but the specific values chosen have no numerical impact. The thickness of this boundary is $1 \times 10^{-30} \mathrm{~m}$ and the conditions are held constant by NUFT. Thus, the very small thickness of the upper boundary renders the value of thermal conductivity at the upper boundary as being inconsequential. These values are used in 2.2.2.3 through incorporation in the files: $d k m$-afc$1 D d s-m c-l i-01, d k m-a f c-1 D d s-m c-m i-01$, and $d k m-a f c-1 D d s-m c-u i-01$. This assumption does not require confirmation.

\subsubsection{SMT-, DDT-, and SDT-Submodel Boundary Conditions}

The mass density of the upper boundary is assumed to have a value of $1.185 \mathrm{~kg} / \mathrm{m}^{3}$. The large value of mass density is used to treat the upper boundary as a fixed-property boundary. The heat capacity of the upper boundary is assumed to have a value of $1 \times 10^{3} \mathrm{~J} / \mathrm{kg}-\mathrm{K}$. The thermal conductivity of the upper boundary is assumed to have a value of $0.0254 \mathrm{~W} / \mathrm{m}-\mathrm{K}$. These values are used in Sections 2.2.1.3, 2.2.3.2, and 2.2.4.3 through incorporation in the file SDT-1Dds_mi (CRWMS M\&O 2000b [DIRS 149862]).

Rationale: The values used for this boundary condition are required in the NUFT input files, but the specific values chosen have no numerical impact. The thickness of this boundary is $1 \times 10^{-30} \mathrm{~m}$ and the conditions are held constant by NUFT. Thus, the very small thickness of the upper boundary renders the value of thermal conductivity at the upper boundary as being inconsequential. This assumption does not require confirmation. 


\subsection{MATERIAL PROPERTIES}

\subsubsection{Permeability of the Drip Shield and Waste Package}

The drip shield and WP are assumed to be impermeable for the entire duration of the MSTH model simulation.

Rationale: These EBS components will take a long time to fail, and it is beyond the scope of this document to model their failure.

\subsubsection{Tortuosity of Invert Materials}

The assumed value for tortuosity of the invert materials is 0.7 . This assumption is used in all NUFT input files (used throughout).

Rationale: This value is consistent with the tortuosity values for the natural barrier system. This assumption does not require confirmation.

\subsubsection{Satiated Saturation of Invert Materials}

The assumed value for satiated saturation of the invert materials is 1.0 . This assumption is used in all NUFT input files (used throughout).

Rationale: This is an upper bound for this parameter, and is therefore conservative. This assumption does not require confirmation.

\subsubsection{Thermal Conductivity Used in Conduction-Only Submodels}

The thermal conductivity data is provided for both dry and wet conditions. The conduction-only submodels (SDT, DDT, and SMT submodels) cannot explicitly represent the influence of liquid saturation on thermal conductivity. Because the rock is predominately closer to being fully saturated (i.e., wet), it was decided to assume the wet value of thermal conductivity (See Table 19) for all of the conduction-only submodels. This assumption is used in Sections 2.2.1.5, 2.2.3.4 and 2.2.4.5, and has no effect on the results of the MSTH model.

Rationale: This assumption has no effect on the MSTH model results because the influence of liquid saturation is explicitly represented by the LDTH submodels. The MSTHAC methodology (see Section 2.3) automatically corrects for the influence of TH processes (including liquid saturation) on temperature. As long as the SDT and SMT submodels utilize consistent assumptions (and data) concerning the thermal conductivity of the stratigraphic units, then the influence of liquid saturation on temperature is rigorously accounted for. As for the validity of this assumption in the DDT submodel, it is important to note that the DDT submodel is only used for two purposes: (1) calculating the temperature difference between the WP and drip shield and (2) calculating the longitudinal temperature variations along the drift axis. Neither of these 
quantities is influenced by whether wet or dry thermal conductivity is applied in the host rock. This assumption does not require confirmation.

\subsubsection{Saturated-Zone Thermal Conductivity}

The SMT submodel (Section 2.2.1) is the only submodel that explicitly represents the saturated zone (SZ). A description of the distribution of the stratigraphic units below the water table is not available; therefore, there was no available data source for determining the distribution of the stratigraphic units in the SZ portion of the SMT submodel. An assumption is made that the SZ is comprised of the ppl unit because it is the predominant stratigraphic unit at the water table for the 33 drift-scale-submodel locations. At 14 out of 33 drift-scale-submodel locations, the pp 1 unit is at the water table. Used in Section 2.2.1.

Rationale: The pp1 unit is the predominant unit at the water table of the 33 drift-scale-submodel locations (with 14 out of 33 locations having the pp 1 unit at the water table). Furthermore, for the 5 stratigraphic units (pp4, pp3, pp2, pp1, bf3, and bf2) that reside at the water table in the 33 drift-scale-submodel locations, the average wet thermal conductivity is $1.375 \mathrm{~W} / \mathrm{m}-\mathrm{K}[1.375=$ $(1.21+1.26+1.26+1.33+1.83+1.36) / 6]$, which is very close to the value of wet thermal conductivity in the ppl unit $(1.33 \mathrm{~W} / \mathrm{m}-\mathrm{K})$. The output of this report is not sensitive to this assumption, and therefore this assumption does not need confirmation.

\subsubsection{Thermal Conductivity and Density for the Active Fracture Model}

The thermal conductivity and density values of the fracture and matrix are apportioned by the following

$$
\begin{aligned}
& \text { fracture conductivity }=\text { total conductivity } \times(\text { fracture porosity }) \\
& \text { matrix conductivity }=\text { total conductivity } \times(1-\text { fracture porosity }) \\
& \text { fracture density } \\
& \text { matrix density }
\end{aligned}
$$

Rationale: There is no commonly accepted approach to apportioning fracture and matrix conductivity and density. However, it is important to note that we conserve the total value of conductivity and the total value of density. Therefore, the total conductive heat flow is the same as a single continuum with the same total value of thermal conductivity. Similarly, during the transient (heat-up) period, we honor the correct mass density of the rock mass. This assumption has no impact on this model. This method is used in all submodels (used throughout). This assumption does not require confirmation.

\subsubsection{Thermal Conductivity, the Lower and Upper Invert Layer}

The thermal conductivity of the crushed tuff invert material was taken from the Repository Subsurface Design Information to Support TSPA-SR. Input Transmittal PA-SSR-99218.Tc (CRWMS M\&O 2000f [DIRS 149137], p. 23). The specific heat capacity and density for the crushed tuff properties were taken from Repository Subsurface Design Information to Support 
TSPA-SR. Input Transmittal PA-SSR-99218.Ta (CRWMS M\&O 1999d [DIRS 105663], Item 2 pp. 13-14) . Used in Section 2.2.2.5.

These data from unqualified sources are assumed to adequately describe the thermal conductivity, specific heat capacity and density of the crushed tuff invert material in the TH model. This assumption is justified because the data are the best available and were developed by applying sound engineering judgment. Any variations in the data that may occur as the qualification process is advanced are not expected to be substantial enough to impact model conclusions.

For this Calculation Report, the entire invert is assumed to have a thermal conductivity $K_{\text {th }}$ of $0.15 \mathrm{w} / \mathrm{mK}$, which is the value of $K_{\mathrm{th}}$ for pure crushed Tuff. The steel beams in the invert are assumed to have a negligible influence on $K_{\text {th }}$. Because the $K_{\text {th }}$ of the steel beams is much greater than that of the invert, this assumption is conservative with respect to predicting peak temperatures on the WP and drip shield. This assumption is used in all LDTH and DDT submodels.

Rationale: The value of $K_{\text {th }}$ in the invert is applied to the DDT submodels as well as to the LDTH submodels. An important function of the DDT submodels is to predict the WP-to-WP variability of temperature conditions along the emplacement drifts. It is important not to over predict axial attenuation of WP-to-WP temperature variability of (by virtue of the choice of $K_{\mathrm{th}}$ in the upper invert layer in the DDT submodels). Therefore, using the $K_{\text {th }}$ for pure crushed Tuff for the entire invert is conservative with respect to predicting WP-to-WP variability in temperatures in the DDT submodels. Using the $K_{\mathrm{th}}$ of pure crushed Tuff for the entire invert is conservative with respect to predicting peak temperatures on the WP and drip shield in the DDT and LDTH submodels. A sensitivity study of the invert $K_{\mathrm{th}}$ found the influence of this assumption to be negligible (BCS 2001d, Section 5.3.1.4.10).

\subsection{Heat Generation and Heat Transfer}

\subsubsection{Constant Heat Flux}

The geothermal heat flux is constant (i.e., does not change with time).. This assumption allows a simplification in the heat transfer equations.

Rationale: This assumption has no impact on this model, and does not require confirmation. Used in Section 2.2.2.3. 


\subsubsection{Effective Thermal Conductivity for Approximating the Influence of Thermal Radiation for DDT Submodels}

The effective thermal conductivity for approximating the influence of thermal radiation in the drift cavity between the drip shield and drift wall is obtained from Francis (2001 [DIRS 155321]). It is assumed that the data contained in DTN SN9908T0872799.004 adequately approximates the influence of thermal radiation between the drip shield and drift surfaces (i.e., drift wall and drift floor), using a time-dependent effective thermal conductivity. This assumption is only used for times greater than $10,000 \mathrm{yr}$ in the DDT submodels for the LTOM.

Rationale: The justification for this assumption is that these data are the only available source for these parameters and were developed using technically sound measurement and analytical methods.

\subsubsection{Effective Thermal Conductivity Approximating the Influence of Thermal Radiation In the Drift for LDTH Submodels}

For the preclosure LDTH submodels, a different effective thermal conductivity than the one described in Section 3.3.3 is used for the drift cavity between the waste package and drift wall. It is assumed that the data contained in the unqualified DTN SN9907T0872799.002 (TBV 3975) adequately describe the time-dependent thermal effective conductivity $K_{t h}$,eff of the air in the emplacement drift. The data contained in that DTN were developed from unqualified sources that do not meet current data quality requirements. This assumption is only used for preclosure LDTH submodels.

Rationale: The justification for this assumption is that these data are the only available source for these parameters and were developed using technically sound measurement and analytical methods. This assumption has no effect on postclosure MSTH-model results.

\subsubsection{Effective Thermal Conductivity Approximating the Influence of Buoyant-Gas- Phase Convection On Heat Transfer In the Drift for LDTH Submodels}

The effective thermal conductivity for approximating the influence of buoyant-gas-phase convection on heat transfer in the drift cavity between the drip shield and drift wall is obtained from Francis (2001 [DIRS 155321]). It is assumed that the data contained in DTN SN9908T0872799.004 adequately approximates the influence of buoyant-gas-phase convection on heat transfer between the drip shield and drift surfaces (i.e., drift wall and drift floor), using a time-dependent effective thermal conductivity. This assumption is used for all postclosure LDTH submodels.

Rationale: The justification for this assumption is that these data are the only available source for these parameters and were developed using technically sound measurement and analytical methods.

\subsection{Geometric description of the EBS and material properties}

Repository layout parameters, which include the footprint, waste package dimensions and drift geometry outlined in Sections 3.4.1 through 3.4.8 below are from CRWMS M\&O 2000g [DIRS 
151467] and 1999e [DIRS 124630]. The assumed parameters represent the repository design in effect at the time this document was developed and are the best available information for the purpose of TH modeling.

\subsubsection{Repository Footprint}

The coordinates of the drift endpoints are given in the file dft1.dat. The HTOM layout is given in the design analysis Site Recommendation Subsurface Layout, ANL-SFS-MG-000001 Rev00 ICN 01 (BSC 2001h [DIRS 154477]). The LTOM layout is given in the design analysis LowerTemperature Subsurface Layout and Ventilation Concepts, ANL-WER-MD-000002 Rev00 (BSC 2001g [DIRS 154554]). These coordinates were used to develop the repository footprint representation used in the SMT submodel (Section 2.2.1.1), and to generate the 33 LDTHsubmodel locations.

Rationale: This is a representative layout of the repository, the actual footprint may vary within the confines of this overall layout. This assumption does not require confirmation.

\subsubsection{Waste Package Lengths and Sequencing}

The waste package (WP) lengths and WP sequencing is taken from the Design Input for the Engineered Barrier System Environment and Barriers (BSC, 2001c [DIRS 154461]). Figure 25 shows the WP lengths, and WP sequencing (including WP-to-WP spacing) that is used in this AMR.

Rationale: This is a representative layout of the waste package lengths and spacing. This assumption does not require confirmation.

\subsubsection{Drip Shield Radius}

The portion of the drip shield above the centerline of the WP has an inside radius of $1.256 \mathrm{~m}$ This information is found in the Subsurface Facility System Description Document (CRWMS M\&O 2000g, Section 1.2.1.5. This information is used in the LDTH submodels (Section 2.2.2) and in the DDT submodels (Section 2.2.4).

Rationale: This assumption is a representation of the drip shield, the final version may vary within the confines of these general parameters. This assumption does not require confirmation.

\subsubsection{Location of Waste Package}

The WP centerline is $1.883 \mathrm{~m}$ above the bottom of the drift and $0.867 \mathrm{~m}$ below the springline. This information is found in the Subsurface Facility System Description Document (CRWMS M\&O 2000g, Section 1.2.1.5).. This information is used in the LDTH (Section 2.2.2) and DDT submodels (Section 2.2.4).

Rationale: This assumption depicts the general location of the waste package within the confines of the emplacement drift and may vary in the final configuration. This assumption does not require confirmation. 


\subsubsection{Waste-Package Diameter}

The WP outer diameter is $1.67 \mathrm{~m}$ and is taken as the average diameter for the inventory from the Enhanced Design Alternative (EDA) II Repository Estimated Waste Package Types and Quantities. (CRWMS M\&O 1999b [DIRS 128028] Item 1 pp. 25-26) (TBV-4902). This information is used in the DDT submodels (Section 2.2.4).

Rationale: This assumption only influences two aspects of the MSTH model: (1) the temperature difference between the waste package and drip shield and (2) the waste-package-to-wastepackage variation of this temperature difference. Note that this temperature difference depends on the waste-package heat output. The 21-PWR and 44-BWR CSNF waste packages, comprising nearly all waste packages with an appreciable heat output (CRWMS M\&O 1999b [DIRS 128028], Worksheet $3 \mathrm{p.18}$ ), have diameters of $1.564 \mathrm{~m}$ and $1.594 \mathrm{~m}$, respectively (CRWMS M\&O 1999b [DIRS 128028], Table 8 p. 25), which are very close to the value of $1.67 \mathrm{~m}$ in the DDT submodels; these waste packages also comprise a large portion (71.3 percent) of the overall waste-package inventory (CRWMS M\&O 1999b [DIRS 128028],Worksheet 3 p. 18). Waste packages that deviate more from this average diameter, such as the 24-BWR 1.238-m-diameter CSNF waste packages and the 5-DHLW/DOE 2.03-m-diameter co-disposal waste packages, generate much less heat and also comprise a relatively small portion of the overall waste-package inventory. Therefore, the assumed diameter of $1.67 \mathrm{~m}$ is very close to the actual diameter for the majority of waste packages in the overall inventory and is also very close to the diameter of the waste packages generating an appreciable temperature difference between the waste package and drip shield. This assumption does not require confirmation.

\subsubsection{Gap Between Waste Package and Drip Shield}

The gap between the top half of the WP and the drip shield is $0.405 \mathrm{~m}$. This information can be determined from the Subsurface Facility System Description Document (CRWMS M\&O 2000g, Section 1.2.1.5). This information is used in the DDT submodels (Section 2.2.4).

Rationale: This assumption depicts the average case for spacing, actual spacing may vary within the overall confines of the assumption. This assumption needs to be consistent with the wastepackage-diameter assumption (Section 3.4.5). Because thermal radiation is the dominant mode of heat transfer between the waste package and the drip shield, the gap between the waste package and drip shield has little influence on predicted behavior in the MSTH model. This assumption does not require confirmation.

\subsubsection{Gap Between Waste Package and Invert}

The gap between the bottom of the WP and the invert is $0.242 \mathrm{~m}$. This information can be determined from the Subsurface Facility System Description Document (CRWMS M\&O 2000g, Section 1.2.1.5). This information is used in the DDT submodels (Section 2.2.4).

Rationale: This is an overall representation of this dimension. This assumption does not require confirmation. 


\subsubsection{Invert Height}

The top of the invert is $0.806 \mathrm{~m}$ above the bottom of the drift. This information is found in the Subsurface Facility System Description Document (CRWMS M\&O 2000g, Section 1.2.1.5). This information is used in the LDTH (Section 2.2.2) and DDT submodels (Section 2.2.4).

Rationale: This is an overall representation of the invert. This assumption does not require confirmation.

\section{USE OF SOFTWARE}

A complete list of the software and the associated software tracking number (STN) is listed in Table 3. Each is designated by type of software: software, routine or macro.

\subsection{Description of Software}

All unqualified software codes used in the preparation of this document are under software configuration management in accordance with AP-SI.1Q, Software Management [DIRS 154418] and have associated software tracking numbers. The names and software tracking numbers for the unqualified codes used in this document are NUFT v3.0s (NUFT, STN: 10088-3.0s-00), RADPRO v3.22 (RADPRO, STN: 10204-3.22-00), XTOOL v10.1 (XTOOL, STN: 10208-10.100), and MSTHAC v6.4.2 (MSTHAC, STN: 10419-6.4.2-00). The software described in this section is used in the following data-flow diagrams (Figure 3 through Figure 20). A more detailed description of these diagrams is given in following sections of this AMR. The computer software used was run on computers located in Lawrence Livermore National Laboratory.

\subsubsection{NUFT v3.0s}

NUFT v3.0s is classified as an unqualified software program (per AP-SI.1Q, Software Management [DIRS 146376]), and was obtained from configuration management. NUFT was run on a Sun Ultra 10 workstation with a SunOS 5.6 operating system. NUFT is used to predict the conditions in the EBS and natural barrier system. NUFT is appropriate for this task.

\subsubsection{RADPRO v3.22}

RADPRO v3.22 is classified as an unqualified software program (per AP-SI.1Q, Software Management [DIRS 146376]), and was obtained from configuration management. RADPRO was run on a Sun Ultra 10 workstation with a SunOS 5.6 operating system. RADPRO is used to predict the radiative heat transfer in the drift. RADPRO is appropriate software for this task.

\subsubsection{XTOOL v10.1}

XTOOL v10.1 is classified as an unqualified software program (per AP-SI.1Q, Software Management [DIRS 146376]), and was obtained from configuration management. The output from XTOOL is graphical (no actual data is produced with XTOOL). XTOOL is tracked in accordance with AP-SI.1Q because it is not commercial software. XTOOL is used to develop graphical representations of the results in the NUFT output files (XVIII-files: *.out). XTOOL is 
appropriate software for this task. Software programs used to produce figures that are not used for model validation are exempt from AP-SI.1Q requirements. XTOOL was run on a Sun Ultra 10 workstation with a SunOS 5.6 operating system.

\subsubsection{MSTHAC v6.4.3}

MSTHAC v6.4.3 is classified as an interim unqualified software program (per AP-SI.1Q, Software Management [DIRS 146376]) and has the following associated STN: 10419-6.4.3-00. MSTHAC 6.4.3 was obtained from configuration management. MSTHAC v6.4.3 was run on a Sun Ultra 10 workstation with a SunOS 5.6 operating system. MSTHAC v6.4.3 is used to predict the multiscale TH conditions based on the various NUFT submodel runs. MSTHAC v6.4.3 is appropriate software for this task. It now incorporates the former SplitEXT v1.0 routine.

\subsection{Description of Routines and Macros}

All routines and macros used in the preparation of this document are qualified in this document or have associated software tracking numbers. The routines qualified in this document are given in Table 7 and are discussed below.

\subsubsection{CONVERTCOORDS V1.1}

CONVERTCOORDS $v 1.1$ is classified as routine per AP-SI.1Q [DIRS 146376], has the following associated STN: 10209-1.1-00, and was obtained from configuration management. CONVERTCOORDS is used to convert from Universal Transverse Mercator coordinates to Nevada State Plane coordinates, as well as to reformat the data (CRWMS M\&O 2000b [DIRS 149862], files: *.inf). The desired format is columns of data, with the input files in a matrix format. CONVERTCOORDS is appropriate software for this task. CONVERTCOORDS was run on a Sun Ultra 2 workstation with a SunOS 5.6 operating system.

\subsubsection{YMESH v1.53}

YMESH v1.53 is classified as a routine per AP-SI.1Q [DIRS 146376], has the following associated STN: 10172-1.53-00, and was obtained from configuration management. YMESH is used in this model to interpolate the thickness of the stratigraphic units at given locations. The input file for YMESH is LBL99-YMESH (CRWMS M\&O 2000b [DIRS 149862]). The output from this software is column.dat (CRWMS M\&O 2000b [DIRS 149862]). YMESH is appropriate software for this task. YMESH was run on a Sun Ultra 2 workstation with a SunOS 5.6 operating system.

\subsection{3 makeColumns v1.1}

The routine makeColumns is classified as a routine per AP-SI.1Q [DIRS 146376] and is currently under qualification review. It creates a family of YMESH input files to generate a family of chimney meshes at a set of specified locations. The input files to makeColumns are column_template and column.data (CRWMS M\&O 2000b [DIRS 149862]). This routine is qualified and is appropriate for its purpose. Routine makeColumns was run on a Sun Ultra 2 
workstation with a SunOS 5.6 operating system. Routine makeColumns_2001 was developed using a $\mathrm{C}++$ compiler.

\subsection{4 define_EBS_fineGrid v1.4}

The routine define_EBS_fineGrid v1.4 is classified as a routine per AP-SI.1Q [DIRS 146376] and is currently under qualification review. Its purpose is to convert NUFT genmsh files, created with YMESH, to separate sets of "matrix" and "fracture" mesh definition instructions, and to add a mapping of materials for the EBS to the mesh. Input files for the routine are *.nft (CRWMS M\&O 2000b [DIRS 149862]). Output files are *.nft.msh.dkm.m, *.nft.msh.dkm.f, *.nft.msh.dkm0, *.nft.msh.dkm0.m, and *.nft.msh.dkm0.f (CRWMS M\&O 2000b [DIRS 149862]). Define_EBS_fineGrid is qualified and is appropriate for its purpose. Routine define_EBS_fineGrid was run on a Sun Ultra 2 workstation with a SunOS 5.6 operating system. Routine define_EBS_fineGrid was developed using a C++ compiler.

\subsection{5 readsUnits v1.0}

Routine readsUnits $\mathrm{v} 1.0$ is classified as a routine per AP-SI.1Q [DIRS 146376] having STN 10602-1.0-00. It converts YMESH column type output to NUFT comments indicating for each rock layer how thick the layer is for a given column. It adds together the widths of adjoining zones of the same material, if any. The input to readsUnits is a file results and the output is called results.units (CRWMS M\&O 2000b [DIRS 149862]). Routine readsUnits is qualified and is appropriate for its purpose. Routine readsUnits was run on a Sun Ultra 2 workstation with a SunOS 5.6 operating system. Routine readsUnits was developed using a $\mathrm{C}++$ compiler.

\subsection{6 addLayers $\mathrm{v} 1.0$}

The routine addLayers v1.0 is classified as a routine per AP-SI.1Q [DIRS 146376] having STN 10603-1.0-00. Routine addLayers modifies NUFT mesh input data by adding an atmosphere or "atm" layer to the top and a water table or "wt" layer to the bottom of the zones for a chimney. The atm layer has depth zero, and the wt layer falls below the lowest rock layer. The routine is qualified and is appropriate for its purpose. Routine addLayers was run on a Sun Ultra 2 workstation with a SunOS 5.6 operating system. Routine addLayers was developed using a C++ compiler.

\subsection{7 addlay $\mathbf{1} 1.0$}

Routine addlay v1.0 is classified as a routine per AP-SI.1Q [DIRS 146376] having SMN Number 10604-SUN-1.0-00 and SMR 10604-SMR-1.0-00. Routine addlay v1.0, like addLayers, reads a NUFT genmsh file generated by YMESH and inserts an atmospheric and a water table layer at the top and bottom of the mesh. In addition, it removes the five lines of NUFT instructions preceding the vertical zone thickness definitions and the final closing parenthesis. Routine addlay demonstrated correct behavior relative to its objective. Routine addlay was run on a Sun Ultra 2 workstation with SunOS 5.6 operating system and was developed using a $\mathrm{C}++$ compiler. 


\subsection{8 columninfiltration v1.2}

Routine columnInfiltration v1.2 is classified as a routine per AP-SI.1Q [DIRS 146376] and is currently under qualification review. The purpose of this routine is to interpolate the infiltration at a given $(x, y)$ location using a Gaussian weighting function (Section 3.1.3). This routine is qualified and is appropriate for its purpose. This routine was run on a Sun Ultra 2 workstation with a SunOS 5.6 operating system. Routine columnInfiltration was developed using a C++ compiler.

\subsection{9 chim_surf_wt_TP2 v 1.0}

Routine chim_surf_wp_TP2 is classified as a routine per AP-SI.1Q [DIRS 146376] and is currently under qualification review. This routine is a combination of previous software chim_surf_TP (STN: 10613-1.0-00), chim_wt_TP (STN: 10614-1.0-00) and xairtab v1.8 (STN: 10607-1.8-00). The purpose of chim_surf_wp_TP2 is to interpolate the temperature and pressure at the ground surface and at the water table for a given location using the inverse distance cubed method. Routine chim_surf_wt_TP2 also reads the boundary condition file chimney_surface_TP (CRWMS M\&O 2000b [DIRS 149862]) and computes air mass fraction and liquid enthalpy at the surface for each chimney. Routine chim_surf_wp _TP2 was run on a Sun Ultra 2 workstation with a SunOS 5.6 operating system and was developed using a Fortran compiler.

\subsubsection{0 infiltab v1.0}

Macro infiltab v1.0 is classified as a macro per AP-SI.1Q [DIRS 146376] having STN 106081.0-00. It extracts infiltration flux data from the input file infiltration.tex (CRWMS M\&O 2000b) and writes the following 9 output files (CRWMS M\&O 2000b [DIRS 149862]) in matrix format: Median_g.dat, Median_m.dat, Median_p.dat, low_g.dat, low_m.dat, low_p.dat, upper_g.dat, upper_m.dat, and upper_p.dat. Infiltrab.m is qualified and is appropriate for its purpose. Macro infiltab was run on a Sun Ultra 2 workstation with a SunOS 5.6 operating system. Macro infiltab was developed using Matlab.

\subsubsection{1 rock_LDTH v1.0}

Macro rock_LDTH v1.0 is classified as a macro per AP-SI.1Q [DIRS 146376] and is currently under qualification review. Macro rock_LDTH v1.0 is similar to macro rock_SUN v.10 which is classified as a macro per AP-SI.1Q having STN 10327-1.0-00. Macro rock_LDTH includes lithophysal porosity which rock_SUN does not. The purpose is to extract material property data from the 1d_driftscale*.pm files (CRWMS M\&O 2000b [DIRS 149862]) and rewrite it formatted as input for NUFT. Routine rock_LDTH is for a DKM continuum. Macro rock_LDTH was run on a Sun Ultra 2 workstation with a SunOS 5.6 operating system and was developed using Matlab. 


\subsubsection{2 rock_SDT v1.0}

Macro rock_SDT v1.0 is classified as a macro per AP-SI.1Q [DIRS 146376] and is currently under qualification review. Macro rock_SDT v1.0 is similar to macro rock_SUN v.10 which is classified as a macro per AP-SI.1Q having STN 10327-1.0-00. Macro rock_SDT includes lithophysal porosity which rock_SUN does not and rock_SDT is a single continuum model whereas rock_SUN is DKM. The purpose is to extract material property data from the 1d_driftscale*.prn files (CRWMS M\&O 2000b [DIRS 149862]) and rewrite it formatted as input for NUFT. Macro rock_SDT was run on a Sun Ultra 2 workstation with a SunOS 5.6 operating system and was developed using Matlab.

\subsubsection{SMT_surf_bc3 v.1.0}

SMT_surf_bc3 is classified as a routine per AP-SI.1Q [DIRS 146376] and is currently under qualification review. The purpose of SMT_surf_bc3 is to interpolate the temperature at the ground surface for a given location using the inverse distance squared method. It was demonstrated to perform in the expected manner. SMT_surf_bc3 was run on a Sun Ultra 2 workstation with a SunOS 5.5.1 operating system. SMT_surf_bc3 was developed using a Fortran compiler.

\subsubsection{SMT_bot_bc3 v.1.0}

SMT_bot_bc3 is classified as a routine per AP-SI.1Q [DIRS 146376] and is currently under qualification review. The purpose of SMT_bot_bc3 is to interpolate the temperature at the bottom of the model for a given $(x, y)$ location using the inverse distance squared method. It was demonstrated to perform in the expected manner. SMT_bot_bc3 was run on a Sun Ultra 2 workstation with a SunOS 5.6 operating system. SMT_bot_bc3 was developed using a Fortran compiler.

\subsubsection{5 bound $v 1.0$}

Macro bound v1.0 is classified as a macro per AP-SI.1Q [DIRS 146376] having STN 10611-1.000. Bound computes temperatures at $1000 \mathrm{~m}$ below the water table. Macro bound takes as input a thermal conductivity file, tcond_wet.dat, the *.units file generated by readsUnits from YMESH column output (containing material thickness), the temperature_ $S$ surface temperature from LDTH boundary conditions, and the temperature_W water table temperature also from LDTH boundary conditions. It writes the file T_bottom.dat. Macro bound was demonstrated to behave according to expectations. Macro bound was run on a Sun Ultra 2 workstation with a SunOS 5.6 operating system and was developed using Matlab.

\subsubsection{6 heatgenAge $v 1.2$}

Routine heatgenAge v1.2 is classified as a routine per AP-SI.1Q [DIRS 146376] having STN 10612-1.2-00. Routine heatgenAge reads a heat-generation-rate file and performs two transformations on it: (1) aging the fuel by a number of years, skipping ahead a number of years 
in the data set and subtracting that number of years from the times of the remaining entries, and (2) ventilating the system for a number of years, removing a specified fraction of the heat during the aging period. Routine heatgenAge performed as expected. Routine heatgenAge was run on a Sun Ultra 2 workstation with a SunOS 5.6 operating system and was developed using a C++ compiler.

\subsubsection{7 rme6 v1.1}

Routine rme6 v1.1 is classified as a routine per AP-SI.1Q [DIRS 146376] having STN 106171.0-00. It converts world view data files to a format readable by YMESH. The input files to rme6 are tspa99_primary_mesh and UZ99_3.grd (CRWMS M\&O 2000b [DIRS 149862]). The output file is LBL99-YMESH (CRWMS M\&O 2000b [DIRS 149862]). Routine rme6 is qualified and is appropriate for its purpose. Routine rme6 was run on a Sun Ultra 2 workstation with a SunOS 5.6 operating system and was developed using a $\mathrm{C}++$ compiler.

\subsubsection{StudyDomain v1.0}

Routine StudyDomain v1.0 is classified as a routine per AP-SI.1Q [DIRS 146376] and is currently under qualification review. StudyDomain is used to create the study domain of the repository with the 33 chimney locations. Study domain takes information from BSC $2001 \mathrm{~h}$ [DIRS 154477] for HTOM and BSC 2001g [DIRS 154554] and outputs to footprint.dat. Routine StudyDomain is appropriate for its purpose and was run on a Sun Ultra 10 workstation with a SunOS 5.6 operating system and was developed using Matlab.

\subsubsection{Input Assembly scripts (LDTH*, SDT* routines)}

The following twelve input assembly scripts, all v1.0, are classified as routines per AP-SI.1Q [DIRS 146376]: LDTH-01-li-i (STN: 10619-1.0-00), LDTH-01-mi-i (STN: 10620-1.0-00), LDTH-01-ui-i (STN: 10621-1.0-00), LDTH-01-li (STN: 10622-1.0-00), LDTH-01-mi (STN: 10623-1.0-00), LDTH-01-ui (STN: 10624-1.0-00), LDTH-03-li (STN: 10622-1.0-00), LDTH03-mi (STN: 10623-1.0-00), LDTH-03-ui (STN: 10624-1.0-00), LDTH-01-li-v (STN: 106251.0-00), LDTH-01-mi-v (STN: 10626-1.0-00), LDTH-01-ui-v (STN: 10627-1.0-00), LDTH-03li-v (STN: 10625-1.0-00), LDTH-03-mi-v (STN: 10626-1.0-00), LDTH-03-ui-v (STN: 106271.0-00), SDT-01i (STN: 10628-1.0-00), SDT-01 (STN: 10629-1.0-00), and SDT-01v (STN: 10630-1.0-00). These scripts build NUFT input files from component subfiles of various types. The scripts were shown to perform in the expected manner. The scripts were run on a Sun Ultra 2 workstation with a SunOS 5.6 operating system. Input assembly scripts were developed using the c-shell V5.1.1.

\section{CALCULATION}

\subsection{OVERVIEW OF DATA FLOW}

The overall data flow in the MSTH model is shown in the flow chart of Figure 2. The flow charts are compartmentalized by NUFT submodel preparation (Section 5.3), submodel execution 
(Section 5.4), and the execution of MSTHAC for each location in the repository (Section 5.5). The last component (shown in yellow) is data binning and graphical-output preparation.

Table 2 lists the specific calculation inputs and sources and their locations on the flowcharts of Section 2. Table 2 through Table 10 allow for more efficient use of the Sub-flowcharts of Figure 5 through Figure 20. There are three kinds of alpha-numeric codes used in the data flowchart figures.

1. Those starting with an $\mathrm{N}$ represent an operation explained in a footnote that results in the creation of one or more files. These operations have output files, but no input files. Table 4 explains the operations involved.

2. Alpha-numeric codes starting with an $\mathrm{E}$ represent editing type operations. These operations have input and output files. Table 5 explains the operations involved.

3. All other codes represent groups of files that flow from one page to the next. Table 6 lists these files as well as the figures of origin and destination.

4. Table 7 through Table 10 give input/output information for the four NUFT submodels.

5. In all flowcharts and references to files, certain naming conventions are chosen for simplification: ' $0 \mathrm{X}$ ' refers to files labeled ' 01 ' for LTOM and '03' for HTOM; '<column>' refers to a column location (.e.g, '15c3'); '<model>' refers to a NUFT submodel (e.g., 'LDTH'); '<infil>' refers to the infiltration case (e.g., 'u' for upper rate); and '<aml>' indicates files labeled with the appropriate AML. For example, the file 'modprop100_0X_<aml>' refers to the usage of HTOM files modprop100_01_14, modprop100_01_27, modprop100_01_55, and modprop100_01_66 and to the LTOM files modprop100_01_11, modprop100_01_23, modprop100_01_46, and modprop100_01_55. A second example is that '<column>.nft.msh.dkm.f' refers to files at all 33 columns, e.g., $15 c 3 . n f t . m s h . d k m . f$ and $14 c 2 . n f t . m s h . d k m . f$.

\subsection{DATA AND PARAMETERS}

Inputs are outlined in the following sections.

\subsubsection{Geometric Description of the EBS and Material Properties}

The geometric description of the EBS consists of both longitudinal dimensions (along the longitudinal axis of the emplacement drifts) and the vertical and lateral dimensions in a vertical cross-sectional plane that is orthogonal to the longitudinal axis of the drifts.

\subsubsection{Drift Spacing}

Emplacement drifts have an 81-m centerline to centerline spacing which is presented in the Subsurface Facility System Description Document (CRWMS M\&O 2000g [DIRS 151467], Section 1.2.1.5). This information is used in the DDT submodels (Section 2.2.4). 


\subsubsection{Waste-Package Spacing}

There is a 10.6-cm gap between Waste packages for HTOM (Figure 26). Gap sizes are $10.6 \mathrm{~cm}$ for LTOM except around BWR packages (see Figure 27). This information is taken from Design Input for the Engineered Barrier System Environment and Barriers (BSC 2001c [154461]). This information is used in the DDT submodels (Section 2.2.4). This input is appropriately used.

\subsubsection{Drift Diameter}

The diameter of the emplacement drifts is $5.5 \mathrm{~m}$ and is presented in the Subsurface Facility System Description Document (CRWMS M\&O 2000g [DIRS 151467], Section 1.2.1.3).

\subsubsection{Properties of Air at Model Boundaries}

The thermal conductivity of air at $16^{\circ} \mathrm{C}$ (ground surface) is $0.0254 \mathrm{~W} / \mathrm{m}-\mathrm{K}$ (Bolz and Tuve 1973 [DIRS 148520], p. 11, interpolated). The solid density of air at $24.8^{\circ} \mathrm{C}$ (intake to emplacement drifts) is $1.185 \mathrm{~kg} / \mathrm{m}^{3}$ (Bolz and Tuve 1973, p. 11, interpolated). The specific heat of air at $25^{\circ} \mathrm{C}$ (intake to emplacement drifts) is 1006 joules $/ \mathrm{kg}-\mathrm{K}$ (Bolz and Tuve 1973, p. 11). This information is used in the SMT, DDT, and SDT submodels through incorporation in the file $S D T$-1Dds. (Accepted data/established fact, handbook value.) This input is appropriately used.

\subsubsection{Properties of EBS Materials}

Table 16 provides invert properties.

Crushed tuff invert hydrologic material properties for the EBS originate in source DTN:MO0009SEPTIHMP.000.

The WP thermal properties are taken from the calculation Tabulated In-drift Geometric and Thermal Properties Used in Drift-Scale Models for TSPA_SR (CRWMS M\&O 1999e [DIRS 124630], p. 11). The emissivity of the waste package is 0.8 (Avallone and Baumeister, 1987 [DIRS 103508], p. 4-68) and is established fact. The value for carbon steel is used as an approximation. This input is appropriately used.

The drip-shield properties comprised of titanium are discussed in ANSYS Calculations in Support of Enhanced Design Alternatives (CRWMS M\&O 1999a [DIRS 102843], p. 21). The drip shield thermal conductivity is $20.55 \mathrm{~W} /(\mathrm{m} \mathrm{K})$ (ASME 1995 [DIRS 108417], p. 611). The density is $4512 \mathrm{~kg} / \mathrm{m}^{3}$ (ASME 1995 [DIRS 108417], p. 620). The drip shield thermal diffusivity is $8.310 *$ $10^{-6} \mathrm{~m}^{2} / \mathrm{sec}$ (ASME 1995 [DIRS 108417], p. 620). The specific heat capacity of the drip shield corresponding to these properties is $550 \mathrm{~J} / \mathrm{Kg}-\mathrm{K}$.

\subsubsection{Hydrologic and Thermal Properties of Stratigraphic Units}

Each stratigraphic unit has two sets of properties, one for its matrix and the other for its fractures. The matrix properties are permeability, porosity, Van Genuchten $\alpha$ parameter, Van Genuchten $\beta$ parameter, residual saturation, and satiated saturation. The fracture parameters include the six categories used for the matrix of the rock (although the values for the fractures are different) and three additional parameters: active fracture parameter, fracture frequency, and fracture to matrix 
area. The thermal properties include grain density, grain specific heat, wet thermal conductivity, dry thermal conductivity, and tortuosity. There are three infiltration cases (each corresponding to an expected climate) over which the repository is being modeled. There is a set of hydrologic properties for each of these infiltration cases.

The matrix properties, fracture properties, and thermal properties corresponding to the base case infiltration flux are given in the file 1d-driftscale_basecase.xls (LB990861233129.001). Properties corresponding to the upper infiltration flux are given in the file $1 d$ driftscale_upperinf.xls (LB990861233129.002). Properties corresponding to the lower bound infiltration flux are given in the file Id-driftscale_lowinf.xls (LB990861233129.003). The fracture properties were updated in several of the units as given in the file LBO011DSTRAC1.001.doc.

The base case matrix properties are reproduced in Table 17. The base case fracture properties are reproduced in Table 18. Thermal properties are reproduced in Table 19. The tortuosity factor is 0.7. This input is appropriately used.

\subsubsection{Thermal Properties of Air Inside the Drifts}

The thermal conductivity of in-drift air in the DDT submodels is from Fundamentals of heat and mass transfer (Incropera and DeWitt 1996 [DIRS 108184], pg. 839: lines 4-12 of the table). The input data is given as a function of temperature in Fahrenheit that is converted to Celsius and used in the file DDT-EBS-Rev100. This input is appropriately used.

\subsubsection{Thermal Properties of Stratigraphic Units for SMT Submodels}

The uncalibrated thermal properties of the stratigraphic units are given in Table 19. The source of this data is thermal_UZ.xls (LB991091233129.006). The $K_{\text {th }}$ and mass density data was updated in the Tptpul and Tptpll units (the tsw33 and tsw35 model units) on the basis of data (BSC 2001 [DIRS 155195]). This data is edited to create the file SMT-1Dds-mi-flt as described in the editing function E:T1 in Table 5 (see Sub-flowchart 16, Figure 20). This input is appropriately used.

\subsubsection{Effective Thermal Conductivity of Cavities Inside Drifts}

Thermal radiative heat transfer inside cavities within the emplacement drifts can be represented with the use of an effective thermal conductivity, which is given as a function of time. Effective thermal conductivity versus time relationships have been developed and are discussed in Assumption Section 3.2.6. This input is appropriately used.

\subsubsection{Stratigraphy}

The primary input for stratigraphy to map rock materials onto the computational mesh is from two files (CRWMS M\&O 1999c [DIRS 110177], Item 1). The first is tspa99_primary_mesh [renamed, original filename is UZ99_3_3D.mesh (LB99EBS1233129.001)], which is the numerical mesh for the site-scale UZ flow model. The second is UZ99_3.grd (LB99EBS1233129.001), which is the vertices file for that mesh. These two files, which define 
the numerical mesh for the 3-D site-scale UZ-flow model, are used to generate the numerical meshes for each submodel (Sections 2.2.1.2, 2.2.2.2, 2.2.3.1 and 2.2.4.2). This input is appropriately used.

\subsubsection{Infiltration Flux}

Infiltration flux is given for three climate periods: [present climate $(0<\mathrm{t}<600 \mathrm{yr})$, monsoonal climate $(600<\mathrm{t}<2000 \mathrm{yr})$, and glacial climate $(2000<\mathrm{t}<1000000 \mathrm{yr})]$ in the nine files Monsoon*.dat, Glacial*.dat, and Modern*.dat (*represents 1, $\mathrm{m}$ and $\mathrm{u}$ ) (source of these nine files: GS000308311221.005). This input is appropriately used.

\subsubsection{Heat-Generation Rate}

Heat-generation decay curves for each of the MSTH model submodel types are based on information in the file DDT-heats-2.xls (BSC, 2001c, DIRS 154461) which contains the following.

- decay curves for CSNF,

- decay curves for HLW,

- WP sequence.

This information is used by all submodels (Section 2.2) through inclusion in the aforementioned files. This input is appropriately used.

\subsubsection{Boundary Conditions}

Temperature and pressure for the 3-D site-scale UZ-flow model are from CRWMS M\&O 1999c [DIRS 110177] (Item 3 and 4, TBV-3942) and can be found in the file bcs99.dat (LB99EBS1233129.003). This information is used to generate boundary conditions for all submodels (Section 2.2). This input is appropriately used.

\subsubsection{Enthalpy}

Liquid enthalpy at 16 and $17^{\circ} \mathrm{C}$ is $67.19 \mathrm{~J} /$ gram and $71.38 \mathrm{~J} /$ gram, respectively (Keenan et al. 1969 [DIRS 134666], p. 2). Accepted Data is established fact, i.e., is handbook value. This input is appropriately used.

\subsection{SUBMODEL PREPARATION}

Generation of the full MSTH model calculation is dependent upon the four major submodel types (LDTH, SDT, DDT, and SMT) as described in Section 2.2. The quality of the final output is dependent on both the quality of the input data and the self-consistency of the multitude of NUFT simulation runs needed for a single MSTH model abstraction. These demands are met 
through the combination of automated tools for generating NUFT input files, along with a modular input file format making extensive use of the include function of NUFT.

The map of input data described in the previous section through the various stages of model generation is shown in the Sub-flowcharts depicted in Figure 5 through Figure 20. The data manipulation required to prepare the raw input data for use by the NUFT-based submodels includes reformatting, change of units, averaging, and interpolation (various schemes). These operations are applied to the data in a well-defined sequence of steps to assure data consistency between all simulation runs.

\subsubsection{Mesh Generation}

There are two major categories of numerical meshes, corresponding to the mountain- and drift-scale submodels. For the mountain-scale (SMT) submodels, the numerical mesh is generated entirely by YMESH, prior to NUFT run time; using YMESH, the user builds the mountain-scale mesh such that the approximated shape of the heated repository footprint can be accurately represented, with sufficiently fine grid-block definition close to the edges of the repository. For the drift-scale (SDT, LDTH, and DDT) submodels, YMESH is used to generate the information that assigns the vertical distribution of the stratigraphic units as well as the gridblock resolution in the vertical direction. A combination of software scripts and hand editing is required to generate the grid-block spacing and material-type specification for the grid blocks that represents the geometry of the EBS inside the emplacement drifts. For the drift-scale submodels, the genmsh feature of NUFT is utilized, whereby the numerical mesh is built at run time.

Mesh generation covers two main categories: the creation of a numerical grid for the computational algorithm, and the assignment of the proper material type, from natural stratigraphy or engineering design, to each grid block. The majority of the actual mapping operations are performed by YMESH, but additional steps are necessary both prior and subsequent to the use of YMESH. For all practical purposes, these pre- and post-fix operations serve as interface modules to the data pipeline.

The processing steps for generating all the numerical meshes required for the MSTH model are depicted in Figure 5 through Figure 8 and described in the following sections.

\subsubsection{YMESH Input}

YMESH can be executed either in a 3-D sense or in a 1-D sense. For the mountain-scale (SMT) submodel, YMESH produces the 3-D numerical mesh file that is utilized directly by NUFT. For the 1-D and 2-D drift-scale submodels, YMESH produces information describing the 1-D vertical distribution of the grid blocks and material types of each of those grid-block layers; this information is placed in the genmsh block of the NUFT input file. For the 2-D and 3-D drift-scale submodels, the grid-block definition in the second and third dimension is assigned by scripts that the modeler develops the template for.

YMESH requires two inputs in order to generate a NUFT-compatible mesh: 
- User input. This file includes specification of (1) the model domain (i.e., model boundaries), (2) the orientation and depth of the repository horizon, (3) the vertical dimensions (and/or the maximum allowable vertical dimensions) of the grid blocks as a function of distance from the repository horizon. For generating an SMT submodel, the areal location of the repository footprint is accomplished by specifying a prefix to the name of the grid blocks in the repository footprint; different prefixes can be used to specify subareas of the repository.

- World grid. This is the stratigraphy primary input for YMESH to map rock materials onto the computational mesh (called the "user's mesh"). The world grid is a combination of items tspa_primary_mesh, which is the numerical mesh for the site-scale UZ flow model, and UZ99_3.grd, which is the vertices file for that mesh. The files UZ99_3.grd and tspa_primary_mesh are processed by rme6 (Section 4.2.17) to create the file LBL99. $Y M E S H$, which is subsequently used in all YMESH operations for both the mountainand drift-scale submodels.

The data in BSC (2001g) [DIRS 154554] for LTOM and BSC (2001h) [DIRS 154477] for HTOM are used to guide the modeler in approximating the repository footprint (Figure 21) for use in the mountain-scale (SMT) submodel (tspa01.grid01-03 and tspa01.grid03-03). Having chosen a reasonable representation of the repository footprint, the next step is choosing the 33 locations for use in the drift scale submodels (column.data). In this model-building process, the modeler's judgment in producing the input files to YMESH is as important as the quality of the input data in its affect on the quality of the final model result.

Several different output types are available from YMESH. For the purposes of the work at hand, the main YMESH output type is NUFT input, and the secondary output type is rock column description, which is placed into NUFT input files as comment lines. This secondary output is also used in generating Table 20.

\subsubsection{Drift-Scale-Submodel Meshes}

The generation of the drift-scale meshes begins with the creation of the column_template files along with the column.data file (Figure 5 through Figure 8). The column.data information records the locations of the individual 33 locations for the LDTH and SDT submodels (see Figure 22). There are two column_template files, one for LDTH submodels, and one for SDT submodels. In each of these cases they serve the same purpose-to describe the vertical mesh resolution for the submodel type.

The routine makeColumns (Attachment I) reads in the column.data file along with a column_template file to produce one individual YMESH input files for each of the 33 drift-scale submodel locations (<column>.dat). Each of these files is run through YMESH to generate a NUFT genmsh file (<column>.nft) and a rock column file $(<$ column>.col) for its respective submodel type and location. These drift scale mesh files then contain information of both mesh geometry, as well as one-dimensional mapping of the materials in the natural system. (The approach of mapping material in one single dimension for the drift scale model is deemed appropriate due to the comparatively small horizontal extent of these submodels.) 
In both SDT and LDTH submodels, the rock column files are used for informational purposes. The interesting information in extracted by readUnits (Section 4.2.5) and output as NUFT comment lines (<column>.col.units). These files will be included in the same NUFT input files which contain the contents of the corresponding <column>.nft information. As a final step in the production of SDT meshes, the routine addlayers (Section 4.2.6) is run on each of the $<$ column>.nft files to add atmosphere and water table material layers at the top and bottom of the mesh, respectively.

The LDTH <column>.nft files require a more complex set of modifications, because of two additional requirements on these submodels-that they include both the engineered system and hydrologic behavior. The hydrologic modeling of the LDTH submodels utilizes the dualpermeability (DKM) capability of NUFT. The DKM requires mapping of materials for both fracture and matrix continua. To include the engineered system, some of the material mappings of the natural system stratigraphy must be overwritten with mapping to the engineered system materials. Both these tasks are achieved with define_EBS_fineGrid (Section 4.2.4). The files <column>.nft.msh.dkm0.f and <column>.nft.msh.dkm0.m contain DKM format, but no engineered system, and are for use in the initialization runs of the LDTH submodel. The files $<$ column>.nft.msh.dkm0.f and <column>.nft.msh.dkm0.m are for use in the remaining LDTH modeling stages.

The mesh for the DDT submodel is derived from an early stage in the development of the LDTH meshes. The YMESH input file at a single submodel location (15c3) is the starting point for development of the DDT meshes. To develop the pre-closure period file $15 \mathrm{c} 3.03 \mathrm{v}$.nft for the DDT model, the grid divisions in the $\mathrm{z}$ dimension are redefined to accommodate the eventual addition of radiation connections. For the post-closure DDT file ( $l 5 c 3 . n f t)$ the mesh is further refined in order to describe the drip shield. The resulting NUFT input files are then run through addLayers (Section 4.2.6), which, similar to addlay, adds atmosphere and water table material layers.

\subsubsection{Mountain-Scale Submodel Mesh}

The mountain-scale mesh is the entire set of mesh information delivered, with the extrapolation of edges constrained to the needs of the abstraction modeling technique. The various modifications of the tspa01.meshoX-03 files were for the purpose of marking regions of interest in grid block names. These tags had no impact on the data values transferred to the next stage in the pipeline.

The mountain-scale numerical mesh is based on the file tspa01.grid03, which derives itself from the motivating concerns outlined in Sections 2.2.1.1 and 2.2.1.2 (the mesh processing steps for the SMT mesh are shown in Figure 8.). This file is processed by YMESH to create both tspa01-mesh01-03 and tspa01-mesh03-03. The differences in these two outputs are only in the names given to some of the grid blocks. In the latter file, the blocks at the repository horizon were given special names to aid in the later steps of the abstraction algorithm. 


\subsubsection{Material Properties}

The primary function of the processing performed on the material property data inputs is to reformat them in a form compatible for NUFT (see Figure 15 and Figure 16). This task involved both modifying the syntax and format of the file, and providing proper units, as well as proper assignments within the separate matrix and fracture continua simulated by NUFT in DKM simulations (using the LDTH submodels). The material properties of the stratigraphic units and those of the EBS are kept in separate input files to maintain modularity.

The input files for the material properties of the stratigraphic units were processed by the routine rock_LDTH.m (Section 4.2.11), to prepare them for the LDTH submodels. Each set of rock properties (from LB990861233129.001, LB990861233129.002, and LB990861233129.003), corresponding to three infiltration-flux cases (mean, high, and low flux) is processed in this manner. The rock property set used in the thermal conduction submodels is constructed from the mean infiltration file produced for the LDTH submodels by keeping only the wet thermal conduction data (BSC 2001 [DIRS 155195]).

The materials incorporated in the EBS are required only in the LDTH and DDT submodels. The files $d k m$-afc-EBS_Rev100-01 and dkm-afc-EBS_Rev100-03 contain the EBS material properties for the HTOM and LTOM LDTH submodels, respectively. The EBS material properties in the DDT submodels are found in the file DDT-EBS_Rev100. The effective thermal conductivity of air, which is used to represent thermal radiation (Section 3.3.2), is found in the files modprop100_OX_<aml> and modprop_dr-OXv.

\subsubsection{Heat-Generation Rate}

Heat-generation-rate versus time information was extracted into individual heat-generation files in NUFT syntax, as shown in Figure 9 through Figure 11. Incorporating the effects of ventilation and aging of Waste packages into the heat-generation-rate versus time tables is accomplished via the routine HeatgenAge (Section 4.2.16).

\subsubsection{Infiltration Flux}

To prepare the infiltration flux source data for use in the NUFT input file requires several steps. First, the routine CONVERTCOORDS is executed on all source data to transform the coordinate representations from UTM NAD 27 coordinates to Nevada State Plane coordinates. Next, the local infiltration flux is interpolated at each of the drift-scale-submodel locations using a Gaussian weighting function implemented in the routine columnInfiltration (Section 4.2.8). This interpolation is done for each of the three climate states (present-day, monsoonal, and glacial), and for each of the three infiltration-flux cases (mean, high, and low); for each drift-scale submodel location (Figure 22), this results in nine values of infiltration flux. The resulting file, infitration.tex, then contains each of the nine infiltration-flux values for each of the drift-scale submodel locations. These values are then extracted from this file by the routine Infiltab (Section 4.2.10) and written into individual files. The contents of these files are included in the LDTH input file creation scripts (Section 4.2.10). These steps are depicted in Figure 12 and Figure 14.

At the time of LDTH input file creation (i.e. execution of the LDTH input file creation scripts), the three different tables of infiltration flux are used to create a time dependent infiltration flux 
definition. This results in an infiltration flux versus time table with a constant present-day-flux value from 0 to $600 \mathrm{yr}$, an abrupt transition at $600 \mathrm{yr}$ to a constant monsoonal-flux value that lasts from 600 to $2000 \mathrm{yr}$, and an abrupt transition at $2000 \mathrm{yr}$ to a constant glacial-flux value that lasts from 2000 to $1,000,000 \mathrm{yr}$.

\subsubsection{Boundary Conditions}

With respect to the process of building boundary conditions, the four submodel types share certain aspects, as well as having certain distinctions (Figure 13). All submodels have lateral boundaries that are no-heat-flow (or adiabatic) boundaries; the TH (LDTH) submodel also has no-mass-flow lateral boundaries. Thus, the only boundary conditions that are specified in the four submodel types are at the upper and lower boundaries.

The conduction-only submodels, which include the SMT submodel (Section 2.2.1), SDT submodel (Section 2.2.3), and the DDT submodel (Section 2.2.4), only require that temperatures be specified at the boundaries. The TH (LDTH) submodel (Section 2.2.2) requires temperature, pressure, and gas-phase air-mass fraction be specified at the boundaries. All four submodel types have the ground surface as the upper boundary; thus, at a given coordinate location, all four submodels share the same upper-boundary temperature. The three drift-scale submodels (SDT, LDTH, and DDT) have the water table as the lower boundary; thus the three drift-scale submodels share the same lower-boundary temperature. The SMT submodel has a lower boundary that is $1000 \mathrm{~m}$ below the water table.

The drift-scale submodels boundary conditions for temperature and pressure are interpolated using inverse distance cubed weighting (Section 3.1.4), implemented in the routine chim_surf_wt_TP2 (see Sub-flowchart 9, Figure 13). The input files are tspa_primary_mesh and bcs_99.dat, and the output files are wt_PT.out, surface_TP-RH.out and surf_TP.out (CRWMS M\&O 2000b). The additional parameters needed for the LDTH model (air-mass fraction and liquid enthalpy) are computed with chim_surf_wt_TP2 (Section 4.2.9).

For the SMT submodel, the routine bound (Section 4.2.15, Sub-flowchart 9, Figure 13) computes the expected temperatures at $1000 \mathrm{~m}$ below the water table. This procedure is based upon assuring the same heat flux (driven by the geothermal gradient) above and below the water table. The vertical temperature profiles for ambient conditions, as predicted by the SDT submodels at each of the $33 \mathrm{drift}$-scale model locations, are used to extrapolate the temperature to a depth of $1000 \mathrm{~m}$ below the water table. These calculated temperatures are interpolated on the SMT grid using inverse distance squared weighting implemented in routines SMT_surf_bc3 and SMT_bot_bc3 (Sections 4.2.13 and 4.2.14) resulting in the lower boundary conditions for the SMT submodel.

\subsection{SUBMODEL EXECUTION}

Several types of NUFT simulation runs are required to produce a single abstracted output dataset. The NUFT submodel-execution procedure is depicted in Figure 17 through Figure 20. Each submodel type fills a particular set of requirements, as described in Section 2.2.1. These varying needs determine the volume of NUFT submodels to be run for each type of simulation. The most demanding submodels in terms of number of simulation runs required are the LDTH 
and SDT submodels. For this reason, these two submodel types have received the greatest attention in automation tools. In general, these submodel types are first created at a single driftscale-submodel location, the submodel is run and refined until the analyst has deemed it ready for production, then the automation components are refined as necessary and the submodel set is generated.

\subsubsection{LDTH Execution}

Sub-flowchart 13 (Figure 17) illustrates the execution of the LDTH submodel. The LDTH submodels are run at each of the 33 drift-scale-submodel locations for four AMLs (Section 2.2.2). In order to be assured of consistency between the data used in these simulations, a set of scripts were developed to generate the required NUFT input files. When one of these scripts is run, it generates a family of 132 NUFT input files, one for each of the four AMLs at each one of 33 locations. A total of nine such scripts were necessary for the LDTH submodelseach of the three infiltration-flux cases requires one script for each of the three stages of LDTHsubmodel execution: (1) initialization run (to obtain initial ambient conditions), (2) the preclosure simulation, and (3) the post-closure simulation.

The data developed for boundary conditions and infiltration are embedded directly in the generating scripts as parameter arrays. These arrays contain one value for each drift-scale submodel location, which are transcribed into these scripts from their respective source files (Figure 17). The concept of operation of these scripts is that for each output file (i.e. generated NUFT input file), various placeholders in a template file are replaced with data relevant to the given scenario. A single value from the data arrays described above, along with filenames containing the other important parameters for the submodel, constitute the information provided to the template by the script.

The LDTH-submodel template files serve the purpose of providing a skeleton NUFT input file for each of the three stages (initialization, pre- and post-closure) of LDTH-submodel execution and for each of the four AMLs, resulting in a total of 12 LDTH template files. These files contain the description of the components of the numerical mesh in the $\mathrm{x}$ and $\mathrm{y}$ dimensions. Since the LDTH model is $2-D$, the $y$-dimensions is only a single layer. On the other hand, the $x$-dimension is the item in the submodel that determines the drift spacing and thereby determines the effective AML being represented in the submodel.

The NUFT input files generated in this procedure reference mesh files, heat-generation-rate files, material property files, run control parameter files, and output time files via the NUFT include directive. The run control parameters are chosen to meet efficiency needs in the execution of the NUFT simulations, and the output times are chosen to give the required temporal resolution.

Execution of a single LDTH submodel takes place in three stages, each of them feeding into the next one. These stages are initialization, pre-closure, and post-closure. The initialization stage is a numerical simulation of the unperturbed natural system, which is run out until the numerical simulation of the natural system achieves equilibrium. This provides the starting point for the pre-closure simulation, which simulates a ventilated system. 
The NUFT output data files from the pre- and post-closure LDTH simulations are later used as input to MSTHAC (Section 2.3).

\subsubsection{SDT Execution}

Sub-flowchart 14 (Figure 18) illustrates the execution of the LDTH submodel. The MSTHAC methodology (Section 2.3) requires that he SDT-submodel simulations are conducted at all locations and AMLs as the LDTH submodels. The production of the NUFT input files for the SDT submodels is automated in a similar method as for the LDTH submodels, but the task is simpler, largely due to the lack of hydrologic data in the SDT submodels. Three SDT generating scripts are required, one for each stage (initialization, pre-closure, and post-closure) of the submodel execution; and only two template files are required, one for the initialization runs, and one covering both the pre- and post-closure simulations. The script files contain the data concerning boundary conditions, as well as the mesh data for the $\mathrm{x}$ dimension (which determines the AML).

The initialization run is used to provide the starting vertical temperature profile, not only for the pre-closure and post-closure simulation periods in the SDT submodel, but also for the DDT submodels. The pre- and post-closure simulations are almost identical, except that the heat-generation curves for the pre-closure period is reduced to simulate ventilation.

\subsubsection{DDT Execution}

Sub-flowchart 15 (Figure 19) illustrates the execution of the LDTH submodel. The DDT submodel need only be run at a single drift-scale submodel location, as discussed in Section 2.2.4. The 15c3 location (Figure 22) was chosen because it is at the geographic center of the repository area and because the host-rock unit at this location is the tsw35, which is the predominant host-rock unit in the repository; 28 of the 33 drift-scale-submodel locations have tsw35 as the host-rock unit (Table 12). As discussed in Section 2.2.5, it would have been acceptable to have chosen any of the other 33 drift-scale-submodel locations. The MSTH model methodology also only requires DDT-submodel results for a single (i.e. the nominal) AML of the repository. To meet the needs for DDT-submodel results, only two sets of NUFT outputs are necessary: one for the pre-closure period, and one for the post-closure period. This greatly simplifies the task of preparing the input files, so that it is feasible to create them without automation tools (Figure 19).

The DDT submodel represents a much greater level of detail within the drift than the other submodels. This is the only component of the MSTH model strategy that simulates heat transfer by thermal radiation explicitly. The thermal radiative components are prepared for NUFT with the use of RADPRO, which requires both a numerical mesh and additional setup information (provided in the file $D D T<a m l>-O X v$.radin). The mesh is read directly from the respective NUFT input file. The additional file contains information of which combination of surfaces may radiate (to each other), and the emissivity values of the respective surfaces. 


\subsubsection{SMT Execution}

Sub-flowchart 16 (Figure 20) illustrates the execution of the LDTH submodel. The overall mountain scale effects of heat flow are simulated with the SMT submodel. This submodel is prepared without the assistance of automation tools, since only two simulations are required (one pre- and one post-closure simulation), which in turn requires only three NUFT input files: initialization, pre-closure, and post-closure (Figure 20). The initialization file is used for the generation of initial conditions for use in each of the other simulations, and the pre- and postclosure simulations are run independently of each other, with the only difference being the heatgeneration curves, as in the case of the SDT submodels.

The SMT submodel uses the same material properties as the other thermal conduction submodels, except that some additional materials appear in this submodel (in files SMT-1Dds-flt and SMT-1Dds-sz), which are not required for the SDT or DDT submodels. The file SMT-IDds-flt contains the thermal properties for the fault zones. The fault-zone materials are additional material type appearing in the site-scale UZ flow model with property assignments. None of the drift-scale-submodel locations intersected any of the fault zone; therefore the thermal properties for the fault zones were not required in the SDT and DDT submodels. The file SMT-1Dds-sz contains the thermal properties for the saturated zone (SZ). Because the SDT and DDT submodels have a lower boundary at the water table, they do not include the SZ in their respective model domains; therefore, these submodels do not require the $\mathrm{SZ}$ thermal properties. The thermal properties of the pp1 unit are assumed for the entire SZ in the SMT (Section 3.2.5).

\subsection{MSTHAC Submodel Assembly}

MSTHAC assembles all of the output of the NUFT submodels using the procedure outlined below. The files l5c3-DDT<aml>-OXv.EBS.ext, SMT<aml>-0Xv.rep.ext, <column>SDT<aml>-OXv.ext, <column>-LDTH-1Dds_mc<infil>-OXV.f.EBS.ext and <column>-LDTHIDds_mc<infil $>-O X v . m . E B S . e x t$ are the submodel output connections from pre-closure simulations, while $15 c 3-D D T<a m l>-0 X . E B S . e x t, \quad S M T<a m l>-0 X . r e p . e x t, \quad<$ column>$S D T<a m l>-O X . e x t,<$ column>-LDTH-1Dds_mc<infil>-OX.f.EBS.ext and <column>-LDTH$I D d s \_m c<$ infil $>-O X . m . E B S . e x t$ are submodel output from post-closure simulations. Note, here $<$ column $>$ is the column location (e.g., $l 5 c 3$ ), $X$ is the case number ( $I$ for HTOM, 3 for LTOM), <infil> is the infiltration case $(l i, m i, u i)$, and $\langle a m l>$ is the AML loading in MTU's (e.g., 60). The output from assembly are the following files containing the data listed in Table 14 and have the filename pattern of caseX_<infil $\left.\rangle_{-}<v a r\right\rangle_{\text {. ext }}$ where $<$ var $>$ refers to the variable listed in Table 14 (e.g., $T \_d w, R H \_d w, Q e v a p \_d s$, etc).

There are six stages for submodel assembly:

\subsubsection{Stage1: Extract TH data from the all pre-closure submodels.}

On the basis of the requested TH data and the MSTHAC methodology (Section 2.3), specific variables are extracted from specific locations in each of the pre-closure submodels. 
5.5.2 Stage 2: Abstract with MSTHAC TH data from the all pre-closure submodels. Using the TH data that was extracted from the pre-closure submodels (Phase 1), the MSTHAC methodology (Section 2.3) is applied to abstract the requested TH data for the pre-closure period. This results in $600 \mathrm{yr}$ of MSTH model results that corresponds to a scenario with $600 \mathrm{yr}$ of drift ventilation, where the nominal heat-generation rate is reduced by $70 \%$ for HTOM and $80 \%$ for LTOM.

\subsubsection{Stage 3: Extract TH data from the all post-closure submodels.}

On the basis of the requested TH data and the MSTHAC methodology (Section 2.3), specific variables are extracted from specific locations in each of the post-closure submodels.

\subsubsection{Stage 4: Abstract with MSTHAC TH data from the all post-closure submodels.}

Using the TH data that was extracted from the post-closure submodels (Phase 3), the MSTHAC methodology (Section 2.3) is applied to abstract the requested TH data for the post-closure period. This results in MSTH model results from $50 \mathrm{yr}$ (for HTOM) or $300 \mathrm{yr}$ (for LTOM) to $1,000,000 \mathrm{yr}$ that corresponds to a scenario with $50 \mathrm{yr}$ (for HTOM) or $300 \mathrm{yr}$ (for LTOM) of drift ventilation, where the nominal heat-generation rate is reduced by $70 \%$ (for HTOM) $80 \%$ (for LTOM), followed by a $50 \mathrm{yr}$ (for HTOM) or $300 \mathrm{yr}$ (for LTOM) to 1,000,000 yr period where the full nominal heat-generation rate is used.

\subsubsection{Stage 5: Combine the pre-closure and post-closure abstracted TH data.}

The abstracted TH results for the pre-closure period (Phase 3) and the post-closure period (Phase 4) are spliced together, resulting in the $38 \mathrm{TH}$ variables at each of the 610 (HTOM) or 762 (LTOM) repository subdomains for each of the $10 \mathrm{WP}$ locations (Figure 26 for HTOM and Figure 27 for LTOM) for for 99 timesteps, resulting in 18,358,560 (HTOM) or 22,933,152 (LTOM) data points for a given infiltration-flux case.

\subsubsection{Stage 6: Bin the abstracted TH data.}

At each of the 610 (HTOM) or 762 (LTOM) repository subdomains, a binning procedure is used (Section 2.4.5) to obtain the average CSNF WP and the average HLW WP. This Phase results in the $38 \mathrm{TH}$ variables at each of the 610 (HTOM) or 762 (LTOM) repository subdomains for $2 \mathrm{WP}$ groups (CSNF and HLW) for 99 timesteps, resulting in 4,589,640 (HTOM) or 5,733,288 (LTOM) data points for a given infiltration-flux case.

\section{RESULTS}

The results of this calculation report fall under two categories: a sensitivity study and the parameters provided to the TSPA. 


\subsection{Sensitivity Calculations in Support of SSPA, Vol.1}

The sensitivity study covers the eight areas of uncertainty listed in Section 1.1. For each area of uncertainty, Table 20 summarizes the areas of uncertainty by tabulating for each area of uncertainty the sensitive parameter investigated, the range of parameter uncertainty, the base case parameter value, the performance measure, and the effect of parameter uncertainty. The details of each of the eight uncertainty studies are discussed below.

\subsubsection{Sensitivity of TH results to neglecting lithophysal porosity (LDTH)}

Neglecting vapor storage within lithophysal porosity is not expected to have a significant effect on the MSTH model results because of the magnitude of expansion of water as it goes from liquid to vapor phase. One pore-volume of water will result in 1000 pore-volumes of vapor (Ellenwood and Mackey 1962 [DIRS 154867], Plate 4C). The matrix and lithophysal pore volumes are similar, so the combined porosity is roughly double the pore-volume of the liquid that would be vaporized. Therefore, the error introduced by neglecting the vapor storage capacity of lithophysal porosity within the computational elements is the difference of accounting for one- instead of two pore-volumes of vapor (out of the 1,000 pore-volumes of vapor generated) that are not displaced to the next computational element. This results in a 0.1 percent over calculation of the amount of vapor displaced to the next zone in the numerical model.

The uncertainty introduced by neglecting this 0.1 percent of the vapor displaced is less than would result from not accounting for the porosity heterogeneity among zones of the numerical model. The range of porosity for the repository units is from 10 to 60 percent (Mongano et al. 1999 [DIRS 149850], p. 17), a six-fold range of porosity. Thus, porosity averaged over zones in the numerical model would have a greater impact on storage than would neglecting lithophysal porosity (one-fold versus six-fold effects).

\subsubsection{Impact of mountain-scale buoyant gas-phase convection on temperature (LDTH)}

Sensitivity analyses were performed (Buscheck 2001a [DIRS 155012]) to consider a range of bulk permeability $\left(\mathrm{k}_{\mathrm{b}}\right)$ values that incorporated plus or minus one $\left(\right.$ low- $\mathrm{k}_{\mathrm{b}}$ and high- $\mathrm{k}_{\mathrm{b}}$ ) and two (very low- $k_{b}$ and very high- $k_{b}$ ) standard deviations around the mean. For these analyses, the permeability distribution was isotropic. Since vertical anisotropy restricts buoyancy, the use of an isotropic permeability distribution provides an upper bound of the possible effects of buoyancy for any given value of permeability. Analyses by Phillips (1991 [DIRS 140641], p. 145) indicate that the critical Raleigh number is smaller when the ratio of permeability in the horizontal direction to the vertical direction is low, which may occur in some regions of the welded fractured units. The influence of vertical anisotropy is not directly addressed by the sensitivity analyses discussed in this section.

Figure 29a shows liquid flux calculations for the HTOM at $5 \mathrm{~m}$ above the drift for a central drift location. This location was chosen to be consistent with locations where seepage had been evaluated in past analyses (CRWMS M\&O 2000d [DIRS 153363], Section 3.2.3.1.5). In the low- $k_{b}$ case, these flux values represent the case in which buoyant gas-phase convection has a negligible influence on thermal-hydrologic behavior. The high- $\mathrm{k}_{\mathrm{b}}$ case is more conducive for buoyant gas-phase convection to be significant. The mean $k_{b}$ case is one in which the influence 
of buoyant gas-phase convection is modest, but not negligible. After about 20,000 years, there is no significant difference between the flux for the three cases. This indicates that the model results are not sensitive to considerations of buoyant gas-phase convection at late times when temperatures approach ambient conditions.

Figure 30 shows the results for the LTOM analyses under the same conditions used in the HTOM analyses. The stairstep responses indicate the impacts of different climates from 0 to 600 years and from 600 to 2,000 years. Results for the LTOM do not show the significant differences between the low, mean, and high- $k_{b}$ cases in contrast to the HTOM analyses from 1,000 to 2,000 years. This is mainly because, without boiling, there is much less water mobilized by vaporization and condensation. These analyses indicate the same long-term flux responses as the HTOM calculations: about $22 \mathrm{~mm} / \mathrm{yr}$, with a difference of less than $1 \mathrm{~mm} / \mathrm{yr}$ among the low, mean, and high- $\mathrm{k}_{\mathrm{b}}$ cases.

Heat mobilized liquid flux $5 \mathrm{~m}$ above the drift is more sensitive to buoyant gas-phase convection than it is at $1 \mathrm{~m}$ above the drift (compare Figure 29a and b). Analyses discussed in Section 5.3.1.4.2 of the SSPA, Vol. 1 (BSC 2001d [DIRS 155950]) indicate that seepage does not occur into drifts during the active boiling period. This is consistent with Figure $29 \mathrm{~b}$ which shows that there is no liquid flux $1 \mathrm{~m}$ above the drift in the HTOM case until after 700 years for the very-high and mean- $\mathrm{k}_{\mathrm{b}}$ cases. At that point, the sensitivity to buoyant gas-phase convection is again apparent. Around 1,000 years, there is greater flux in the very high- $\mathrm{k}_{\mathrm{b}}$ case $(52 \mathrm{~mm} / \mathrm{yr})$ than in the mean- $\mathrm{k}_{\mathrm{b}}$ case $(32 \mathrm{~mm} / \mathrm{yr})$, indicating some sensitivity of liquid flux to buoyant gas-phase convection. However, this amount of flux is about the same magnitude as the ambient flux range and less than the long-term flux from the wetter climates (up to $100 \mathrm{~mm} / \mathrm{yr}$ ). There is no significant difference in liquid-phase flux at 5-m and $1-\mathrm{m}$ above the drift in the LTOM (Figure 30a and $\mathrm{b}$ ), reflecting the minimal thermal mobilization of water.

\subsubsection{Sensitivity to host rock bulk permeability (LDTH)}

Sensitivity analyses were performed to consider the impacts of uncertainties in the values of fracture permeability used in the MSTH model. In the MSTH model, fracture and matrix are handled as the separate but overlapping continua (dual-permeability model) in which the permeability of the matrix and the fractures are expressed as different bulk permeabilities applied uniformly to the rock mass. Since the actual fracture permeability is heterogeneous, applying bulk permeability to the heterogeneous system could introduce uncertainties in the results. Because the fracture permeability rather than the matrix permeability (which is significantly smaller) dominates the TH processes, the practice has been to refer to the bulk permeability $\left(\mathrm{k}_{\mathrm{b}}\right)$ without distinguishing between the matrix and fracture permeability. Thus, the term "bulk permeability" actually refers to the bulk fracture permeability. This practice will be followed throughout this section.

Sensitivity analyses (Buscheck 2001a [DIRS 155012]) were designed to address the potential for impacts by comparing the results over a wide range of fracture properties. The first analysis considered the impacts that a range of $k_{b}$ representing approximately one standard deviation above and below the mean values (identified as high, mean, and low $k_{b}$ ) had on both temperatures and relative humidity on the drift wall and drip shield in the center of the repository (CRWMS M\&O 2000b [DIRS 149862)], Figure 5-2, location 15c3) for the HTOM with the mean 
infiltration flux. Analyses were also conducted for two standard deviations above and below the mean values (identified as very high, mean, and very low $k_{b}$ ). Although the analyses were for the $15 \mathrm{c} 3$ location, they are applicable to the portion of the repository in which the host rock is the Tptpll unit, which comprises more than three-quarters of the repository area. The actual values for $\mathrm{k}_{\mathrm{b}}$ used in determining the very high, high, mean, low, and very low $\mathrm{k}_{\mathrm{b}}$ values in the models are shown in Table 21. As can be observed in Figure 31, the temperatures beyond 1,000 years on the drift wall and the drip shield were insensitive to the $k_{b}$ used in the models for the HTOM. A few decades after closure there was some sensitivity of drift wall and drip shield temperature to bulk permeability. The drift wall temperatures were consistent for the mean and low $\mathrm{k}_{\mathrm{b}}$ cases, but slightly higher than the temperature for the high $k_{b}$ case. The drip shield temperatures were not sensitive to differences between mean and low $k_{b}$, while the high $k_{b}$ resulted in a slightly lower temperature. For the very high and high $\mathrm{k}_{\mathrm{b}}$ cases, the peak temperature is $11^{\circ} \mathrm{C}$ and a few ${ }^{\circ} \mathrm{C}$ lower, respectively than in the mean $\mathrm{k}_{\mathrm{b}}$, low $\mathrm{k}_{\mathrm{b}}$, and very low $\mathrm{k}_{\mathrm{b}}$ cases.

The relative humidity results for the drip shield (Figure 31 ) were insensitive to the $k_{b}$ and nearly insensitive for the drift wall; there was less than a 1-percent difference among the five cases. The results suggest that for the mean infiltration case, the MSTH model relative humidity results for the drift wall and drip shield are relatively insensitive to repository-scale permeability variability. This indicates that repository-scale variability of $k_{b}$ will not significantly modify MSTH model predictions of temperature and relative humidity in portions of the repository for which the Tptpll is the host rock unit. The weak dependence of temperature and relative humidity on $\mathrm{k}_{\mathrm{b}}$ in the Tptpll unit indicates that temperature and relative humidity predictions by the MSTH model would also be relatively insensitive to $\mathrm{k}_{\mathrm{b}}$ in portions of the repository where the host-rock unit is other than the Tptpll.

Sensitivity of the MSTH model liquid saturation in the invert material was also evaluated. Figure 32 shows the predicted liquid saturation in the higher-temperature operating model for the same location within the repository footprint as the temperature and relative humidity assessments discussed above. The results are for the upper invert layer directly below the drip shield in the central portion of the drift (Figure 23 shows the computational cells in the invert). The index $i$ in Figure 32 is 4 for the outermost invert cell and 1 for the innermost cell. These analyses were performed for the mean infiltration flux case.

Figure 32 shows that the liquid saturation in the upper invert is zero until about 2,000 years after emplacement for the mean and low $k_{b}$ cases and about 3,000 years for the high $k_{b}$ case. The very high $k_{b}$ case has an onset of rewetting at about 4,000 years. The general trend from these five permeability cases is that the onset of rewetting increases with $k_{b}$ and that the final "steady-state" value of liquid saturation increases with decreasing permeability. As the permeability decreases, liquid saturation in the fractures must increase to accommodate a given percolation flux; the increased liquid saturation results in lower capillary tension in the fractures and adjoining rock matrix. The lower capillary tension in the host rock promotes more wicking of moisture into the crushed-tuff invert. Because the capillary properties of the fractures were not varied along with the permeability and because a single continuum was used to represent the crushed-tuff invert, the trend between these permeability cases should be viewed qualitatively rather than quantitatively. 
A similar set of analyses was performed for the LTOM. These analyses considered the same range of permeability and the same location within the potential repository footprint as can be observed in Figure 33; the temperatures on the drift wall and the drip shield are insensitive to the $\mathrm{k}_{\mathrm{b}}$ bulk permeability used in the models. The relative humidity on the drip shield and drift wall is also insensitive to the permeability. This indicates that repository-scale variability of permeability will not significantly modify MSTH model predictions of temperature and relative humidity in portions of the repository for which the Tptpll is the host rock unit. The weak dependence of temperature and relative humidity on permeability also indicates that temperature and relative humidity predictions by the MSTH model would also be relatively insensitive to permeability in portions of the repository where the host-rock unit is other than the Tptpll.

The relative humidity of the drift wall for the LTOM is always nearly 100 percent (which is the relative humidity for ambient conditions), whereas for the HTOM, relative humidity drops to 20 percent about 10 years after closure and then gradually increases with time until it reaches nearly 100 percent around 1,000 years after emplacement; thus, relative humidity at the drift requires about 1,000 years to return to ambient conditions. For this reason, the results of the MSTH model are not only insensitive to variations in $k_{b}$ of two standard deviations about the mean, but are also conservatively bounded in the maximum relative humidity for this lower temperature operating mode. If there are any effects on the results from permeability much different from the mean permeability value (e.g., due to capillary effects), the results could only reduce the relative humidity. Because relative humidity effects performance at higher values, the results of the MSTH model provide a conservative bound.

The sensitivity of the MSTH model liquid saturation in the invert material was also evaluated for the LTOM. Figure 34shows the predicted liquid saturation at the same location in the repository footprint as the temperature and relative humidity assessments discussed above. The results are for the upper invert layer directly below the drip shield, in the central portion of the drift (Figure 23). These analyses were performed for the mean infiltration flux case. The onset of rewetting occurs earlier in the invert for the LTOM than for the HTOM (compare Figure 34and Figure 32). The same trends between the permeability cases are observed in the LTOM as in the HTOM. The general trend is that the onset of rewetting increases with permeability and that the final "steady-state" value of liquid saturation increases with decreasing permeability. Because the capillary properties of the fractures were not varied along with the permeability of the fractures and because a single continuum was used to represent the crushed-tuff invert, the trend between these $k_{b}$ cases should be viewed qualitatively rather than quantitatively.

\subsubsection{Sensitivity of MSTH results to host rock thermal conductivity (LDTH)}

Sensitivity analyses were performed to consider the uncertainties in drift-wall and drip-shield temperature, and relative humidity, and invert liquid saturation that result from uncertainties in the values of host rock thermal conductivity used in the MSTH model. The MSTH model uses values of bulk host rock thermal conductivity as input. In field and laboratory measurements of host-rock thermal conductivity used in these thermal-hydrologic calculations, there is not discrimination between the relative contributions of the fractures and matrix to the bulk thermal conductivity. However, it is recognized that fractures and void spaces can have a significant impact on the thermal conductivity. It has been also recognized (CRWMS M\&O 2000b [DIRS 149862], Sections 4.1.7 and 5.2.4) that there is a significant difference in thermal 
conductivity between wet (or saturated) rock and dry (or unsaturated) rock. The liquid saturation state of the rock mass must be determined by the MSTH model analyses for any given time. Therefore, there are uncertainties in the value of thermal conductivity to use, which could introduce uncertainties in the MSTH model results.

The thermal conductivity of the rock mass for the Tptpll unit was estimated based upon an evaluation of the lithophysal porosity using two different methods. The first method used the information from mapping the ECRB drift (Mongano et al. 1999 [DIRS 149850]). The second method used information from core data and bulk density from geophysical measurements for borehole USW SD-7 at Yucca Mountain (BSC 2001j [DIRS 155008]). The porosity estimates from descriptive statistics of the ECRB mapping and the calculation of the lithophysal porosity from borehole USW SD-7 are comparable, and show that the mean values for lithophysal porosity are 0.125 and 0.120 , respectively. The second method showed a low degree of correlation among the parameters of matrix saturation, matrix porosity, and air-filled lithophysal porosity.

The Monte Carlo simulation method (Hahn and Shapiro 1967 [DIRS 146529], pp. 237 to 241) was used to calculate expectation and variance of thermal conductivity for the Kunii and Smith (1960 [DIRS 153166], p. 75) predictive relation, the parallel predictive relation, and a composite predictive relation. The composite predictive relation uses the Kunii and Smith predictive relation for matrix properties and the parallel predictive relation (Hadley 1986 [DIRS 153165], p. 914) for the air-filled lithophysal porosity. The results of these calculations for the Tptpll unit (BSC 2001 [DIRS 155008], Section 6) are shown as the high and low cases in Table 22.

Using the values of thermal conductivity determined in the analyses described above (BSC 2001j [DIRS 155008]), the MSTH model sensitivity analyses (Buscheck 2001a [DIRS 155012]) using the LDTH submodel were designed to address the potential for impacts of uncertainty in thermal conductivity by comparing the results of analyses over the range of thermal conductivity values determined. Table 22 reports the values of thermal conductivity used in the models. The high and low values represent those determined by the Monte Carlo simulations and the mean determined from the ECRB measurements, geophysical measurements, and analyses of Sass et al. (1988 [DIRS 100644]).

The first set of sensitivity analyses considered the HTOM impacts. These analyses evaluated that the range of conductivity (the high, mean, and low conductivities shown in Table 22) had on temperature and relative humidity on the drift wall and the drip shield for a location in the center of the repository (CRWMS M\&O 2000b [DIRS 149862], Figure 5-2, location 15c3) for the HTOM case with the mean infiltration flux. Although the analyses were for the $15 \mathrm{c} 3$ location, they are applicable to the portion of the repository in which the host rock is the Tptpll unit, which comprises more than three-quarters of the repository area.

Temperatures on the drift wall and drip shield are sensitive to the thermal conductivity ( $\mathrm{K}_{\mathrm{th}}$ ) used in the models for as long as 100,000 years (Figure 35a and Figure 35b). The MSTH model temperature results are more sensitive to variability in $K_{\text {th }}$ values represented by values lower than the mean than they were to values above the mean. The drift wall peak temperatures for the low $\mathrm{K}_{\text {th }}$ values are nearly $220^{\circ} \mathrm{C}$, compared to almost $150^{\circ} \mathrm{C}$ for the mean. The difference was around $70^{\circ} \mathrm{C}$, which was almost 50 percent higher than the mean value temperatures. The 
difference between the mean and high $\mathrm{K}_{\mathrm{th}}$ temperatures was much less (about $12^{\circ} \mathrm{C}$ ): $136^{\circ} \mathrm{C}$ for the high $\mathrm{K}_{\text {th }}$ compared to $148^{\circ} \mathrm{C}$ for the mean value, which is only 5 percent lower than the temperatures for the mean value. At 1,000 years after emplacement, the difference between the mean and high $\mathrm{K}_{\mathrm{th}}$ results is negligible $\left(2^{\circ}\right.$ to $3^{\circ} \mathrm{C}$ ), with the mean $\mathrm{K}_{\mathrm{th}}$ temperatures around 96 to $97^{\circ} \mathrm{C}$, the boiling temperature of water. This is significant, since at 1,000 years the low $\mathrm{K}_{\mathrm{th}}$ temperatures are $112^{\circ} \mathrm{C}$ and rock at the drift wall would remain above the boiling point for approximately another 1,000 years.

Similar sensitivity to the $K_{\text {th }}$ values used in the MSTH model analyses was observed in temperatures on the drip shield. The peak temperature for the low $\mathrm{K}_{\mathrm{th}}$ was $234^{\circ} \mathrm{C}$, compared to $160^{\circ} \mathrm{C}$ for the mean and $150^{\circ} \mathrm{C}$ for the high $K_{t h}$ values. The differences between the mean calculation results and the low and high $K_{t h}$ calculation results are 46 percent and 6 percent, respectively. Again, the differences persist for up to 100,000 years, while the difference between the mean and high $K_{t h}$ results essentially become insignificant within about 1,000 years. The temperatures drop to the boiling point at about the same time as the drift wall temperatures (approximately 1,500 years and 2,000 years for the mean/high and low $K_{\text {th }}$ values, respectively).

There are significant differences in calculated relative humidity on the drift wall for the low and mean $K_{\text {th }}$ cases (Figure $35 \mathrm{c}$ ). The low $K_{t h}$ results in very low relative humidity in the early postclosure times: it starts at 3 to 5 percent from 50 to 60 years and gradually increases, up to 60 percent at 1,000 years. Relative humidity calculated for the mean value of $K_{t h}$ is 20 percent in the 50- to 60-year time frame, increasing to 60 percent by 400 years. The comparable, time-wise, values for the high $K_{t h}$ are 28 percent and 70 percent, as shown on Table 23.

However, the impact on performance due to the differences in relative humidity is more significant during later times, when humidity exceeds 60 percent. As noted in Table 23 the humidity reaches 60 percent for the mean $\mathrm{K}_{\mathrm{th}}$ within 400 years after emplacement and increases to 95 percent at 1,000 years after emplacement. The relative humidity calculated for the high $\mathrm{K}_{\mathrm{th}}$ case is 70 percent at 400 years (17 percent higher than the mean value results) and nearly 100 percent at 1,000 years. For the high $\mathrm{K}_{\mathrm{th}}$ case, those same relative humidity levels of the mean $\mathrm{K}_{\mathrm{th}}$ case would occur at 280 and 700 years, respectively. The time required for the drift wall to attain ambient (near 100 percent) relative humidity is $900,1,000$, and 2,000 years for the high, mean, and low $K_{t h}$ cases, respectively. If the $K_{\text {th }}$ values were actually lower than the mean used in the performance assessment, the results would be bounding; the relative humidity would be significantly lower for longer periods of time.

The relative humidity results for the drip shield (Figure $35 d$ ) are sensitive to $K_{t h}$ for values of $K_{t h}$ smaller than the mean; however, they are relatively insensitive for values of $K_{\text {th }}$ larger than the mean value used in the MSTH model analyses. The most divergent case, low $\mathrm{K}_{\mathrm{th}}$, has a much lower relative humidity than either the mean or high $\mathrm{K}_{\mathrm{th}}$ case for the first 2,000 years. Beyond 2,000 years, there are minor differences between these cases.

Sensitivity of the MSTH model liquid saturation in the invert material was also evaluated. Figure 36 shows the predicted liquid saturation for the same locations as the temperature and relative humidity assessments discussed above. The results are for the upper invert layer directly below the drip shield in the central portion of the drift. These analyses were performed for the mean infiltration flux case. The liquid saturation in the invert is zero until about 2,100 years 
after emplacement for the mean and high $K_{\text {th }}$ cases and about 3,200 years for the low $K_{t h}$ case. From the onset of rewetting (i.e., onset of nonzero liquid saturation values) until about 13,000 years after emplacement, the liquid saturation within the invert is somewhat sensitive to the $K_{t h}$ used in the model. The difference in liquid saturation at 4,000 years is only 2.6 percent between the high $K_{\text {th }}$ case ( 2.8 percent saturation) and the low $K_{\text {th }}$ case $(0.2$ percent saturation). There is approximately a 2,000-year difference in time for liquid saturation to reach 2 percent, which is nearly 3,000 years for the high $K_{t h}$ case and 5,000 years for the low $K_{t h}$ case. The high and mean $\mathrm{K}_{\text {th }}$ cases are quite similar. This leads to the conclusion that it is likely that only much lower $K_{\text {th }}$ values would significantly change the liquid saturation conditions in the invert for $\mathrm{K}_{\mathrm{th}}$ variations greater than those considered. In the long-term period of the HTOM, there is essentially no difference due to variation in $\mathrm{K}_{\text {th }}$.

A set of analyses similar to those of the HTOM were performed for the LTOM. These analyses using the LDTH submodel considered the same range of thermal conductivity and the same location in the repository footprint as the HTOM analyses.

As can be observed in Figure 37a and Figure 37b, temperatures on the drift wall and the drip shield were sensitive to the $K_{t h}$ used in the models for as long as 100,000 years. The MSTH model temperature results were more sensitive to variability in $K_{t h}$ values (represented by values lower than the mean) than they were to values above the mean. The drift wall peak temperatures for the low $\mathrm{K}_{\mathrm{th}}$ values were approximately $88^{\circ} \mathrm{C}$, compared to $73^{\circ} \mathrm{C}$ for the mean. The difference was $15^{\circ} \mathrm{C}$, which was about 21 percent higher than the mean value temperatures. The difference between the mean and high $\mathrm{K}_{\mathrm{th}}$ temperatures was much less, around $3^{\circ} \mathrm{C}, 70^{\circ} \mathrm{C}$ for the high $\mathrm{K}_{\mathrm{th}}$ compared to $73^{\circ} \mathrm{C}$ for the mean value, which is only 4 percent lower than the temperatures for the mean value. At 10,000 years after emplacement, the difference between the mean and high $\mathrm{K}_{\mathrm{th}}$ results is negligible, while there is a difference of $5^{\circ} \mathrm{C}$ between the low and mean $\mathrm{K}_{\mathrm{th}}$ cases. For the high and mean value cases, the drift wall temperatures drop below $70^{\circ} \mathrm{C}$ at 1,400 years after emplacement, whereas the low $\mathrm{K}_{\mathrm{th}}$ temperatures do not drop below $70^{\circ} \mathrm{C}$ until 3,800 years after emplacement.

A similar sensitivity trend to the $K_{\text {th }}$ values used in the MSTH model analyses was observed in the temperatures on the drip shield. The peak temperature for the low $\mathrm{K}_{\text {th }}$ was $93^{\circ} \mathrm{C}$, compared to $77^{\circ} \mathrm{C}$ for the mean and $75^{\circ} \mathrm{C}$ for the high $\mathrm{K}_{\mathrm{th}}$ values. The differences between the mean calculation results and the low and high $\mathrm{K}_{\mathrm{th}}$ calculation results are 21 percent and 3 percent, respectively. Again, the differences persist for up to 100,000 years, while the difference between the mean and high $K_{t h}$ results essentially become insignificant within about 10,000 years. The temperatures drop to below $70^{\circ} \mathrm{C}$ at approximately 2,000 years and 4,000 years for the mean/high and low $\mathbf{K}_{\text {th }}$ values, respectively.

There are no differences in calculated relative humidity on the drift wall for the low, high, and mean $\mathrm{K}_{\mathrm{th}}$ cases (Figure $37 \mathrm{c}$ ). All cases result in nearly 100 percent relative humidity for all times. The lower temperatures do not result in rock dryout, and the MSTH model analyses do not account for moisture removal by ventilation. Therefore, the relative humidity remains close to 100 percent (ambient conditions) for all times. The relative humidity results for the drip shield (Figure 37d) are very insensitive to the $K_{t h}$ used in the MSTH model analyses. 
Figure 38 shows the predicted liquid saturation for the same locations as the temperature and relative humidity assessments discussed above. The results are for the upper invert layer directly below the drip shield in the central portion of the drift. These analyses were performed for the mean infiltration flux case. The liquid saturation in the invert is zero for all thermal conductivity cases until about 1,100 to 1,200 years after emplacement for the mean and high $\mathrm{K}_{\text {th }}$ cases and about 2,000 years for the low $K_{t h}$ case. From the onset of rewetting until 20,000 years after emplacement, there is a degree of sensitivity of liquid saturation within the invert to the $K_{t h}$ used in the model. The difference in liquid saturation at 7,000 years is 1.8 to 1.9 percent liquid saturation between the high $\mathrm{K}_{\mathrm{th}}$ case (5.1 percent saturation) and the low $\mathrm{K}_{\mathrm{th}}$ case (3.2 to 3.3 percent saturation). There is a 2,000-year difference in time when the invert liquid saturation reaches 3 percent between the high and low $K_{\text {th }}$ cases, which reach 3 percent liquid saturation at 3,000 and 5,000 years respectively. The time difference increases up to 3,000 years for 3.2 percent between the high and low $K_{\text {th }}$ cases.

Comparison of Results of Higher- and Lower-Temperature Operating Modes-Peak temperatures on the drift wall in the central portion of the repository for the HTOM and the LTOM are about 148 and $73^{\circ} \mathrm{C}$, respectively, under mean infiltration and permeability conditions. The temperatures decrease to ambient (i.e., approximately $22.5^{\circ} \mathrm{C}$ ) around 100,000 years. The relative humidity on the drift wall for the HTOM drops to about 20 percent about 60 years after emplacement, then increases to 95 percent at about 1,000 years. The temperatures at the time that the drift wall relative humidity approaches 100 percent are about $90^{\circ} \mathrm{C}$, and decrease to $70^{\circ} \mathrm{C}$ around 4,000 years. Thus, there is a period of about 3,000 years when the relative humidity is nearly 100 percent and temperatures are higher than $70^{\circ} \mathrm{C}$. For the LTOM, the relative humidity is always nearly 100 percent. The drift wall temperatures for the LTOM are between 70 and $74^{\circ} \mathrm{C}$ from about 400 to 1,500 years. Thus, there is a period of about 900 years when the temperatures are slightly above $70^{\circ} \mathrm{C}$ and the relative humidity is nearly 100 percent. For the LTOM, the drip-shield relative humidity is very insensitive to $\mathrm{K}_{\text {th }}$. For the HTOM, drip-shield relative humidity is sensitive to $K_{\text {th }}$ for values less than the mean $K_{\text {th }}$; this sensitivity lasts for about 2,000 years. For values of $\mathrm{K}_{\mathrm{th}}$ greater than the mean value, relative humidity is much less sensitive, with relative humidity being somewhat higher for the high $K_{t h}$ case during the first 1,100 years.

\subsubsection{Sensitivity of MSTH Model Results to Lithophysal Porosity (LDTH)}

Sensitivity analyses of the impact of $\mathrm{K}_{\mathrm{th}}$ uncertainties, largely due to lithophysal porosity impacts on thermal conductivity, were discussed in Section 6.1.4. In addition to the impacts on the thermal conductivity, the lithophysal porosity can also impact the heat capacity. Heat capacity is a function of rock mass density, and therefore subsequently a function of porosity. The heat capacity, thus, will decrease with increased porosity. Mapping data along the ECRB drift walls (Mongano et al. 1999 [DIRS 149850]) indicate that the mean lithophysal porosity of the lower lithophysal unit is 0.125 (see Section 4.3.5.3.2). The matrix porosity of the lower lithophysal unit (tsw35) is 0.115 (DTN: MO9901RIB00044.000 [DIRS 109966]). Because the lithophysal porosity is roughly equal to the matrix porosity, uncertainty in the lithophysal porosity can significantly impact the porosity and the heat capacity used in the MSTH model analyses. 
Sensitivity studies were performed using the LDTH submodel of the Multiscale Thermohydrologic Model (CRWMS M\&O 2000b [DIRS 149862]) to consider the effects of uncertainties in the lithophysal porosity on both thermal conductivity and heat capacity (BSC 2001 [DIRS 155008]), which in turn affect temperature, relative humidity, and liquid saturation at the drift wall and drip shield (Buscheck 2001a [DIRS 155012]). These sensitivity studies were performed for the same repository location as sensitivity studies in Section 6.1.4, which considered the lithophysal porosity on thermal conductivity alone. By comparing the results of the mean, high, and low lithophysal porosity results with the $K_{t h}$ analyses (Section 6.1.4), an assessment can be made of the sensitivity of the results to the lithophysal porosity, or more specifically to the influence of lithophysal porosity on heat capacity. Figure 39 shows the first analysis, which considered the effects that the range of lithophysal porosity (Table 24) had on the temperature and relative humidity on the drift wall and drip shield for a location in the center of the repository (location $15 \mathrm{c} 3$ reference) for the HTOM with the mean infiltration flux. Although the analyses were for the $15 \mathrm{c} 3$ location, they are applicable to the portion of the repository in which the host rock is the Tptpll unit, which comprises more than three-quarters of the repository area.

As can be observed by comparing the results shown on Figure 35a with the results shown on Figure 39a, the peak temperatures on the drift wall were 2 to $3^{\circ} \mathrm{C}$ cooler for the low lithophysal porosity case that included effects of the porosity on the heat capacity than that which did not. Likewise, peak temperatures were $16^{\circ} \mathrm{C}$ higher for the high lithophysal porosity than for the comparable case from Section 6.1.4 that did not include these effects on the heat capacity. Similar, but of smaller magnitude, differences were noted for the temperatures on the drip shield (Figure 35b and Figure 39b). The differences for the drift wall and drip shield disappeared within 1,000 years of emplacement. Relative humidity differences were noted for the drift wall only, and only for the low lithophysal porosity case where there was an approximately 3 percent relative humidity difference when the porosity impacts on the heat capacity were considered (Figure 39c and Figure 35c). There was no impact on the drip shield relative humidity (Figure $39 \mathrm{~d}$ and Figure 35d). Invert saturation (Figure 40) was not affected by neglecting the influence of lithophysal porosity on heat capacity. These observations indicate that neglecting the influence of lithophysal porosity on heat capacity will not add to uncertainty in the results from the MSTH model.

Comparisons of results for the LTOM with and without consideration of the influence of lithophysal porosity on heat capacity reveal no difference. This is seen by comparing results of the analyses of temperatures on the drift wall and drip shield that included consideration of the effects of lithophysal porosity on heat capacity (Figure 41) with the results of the analyses that did not include the influence of lithophysal porosity on heat capacity (Figure 37). There is no difference in any of the curves. This result is consistent with the conclusions for the HTOM.

Figure 42 shows the predicted liquid saturation for the same location as the temperature and relative humidity assessments discussed above. The results are for the upper invert layer that is directly below the drip shield in the central portion of the drift. These analyses were performed for the mean infiltration-flux case. Comparison of Figure 42, which shows the results of analyses that considered the influence of lithophysal porosity on heat capacity, with Figure 38, which does not include the consideration of the influence of lithophysal porosity on heat capacity, indicates 
that there is no impact on the MSTH model invert saturation results as a result of neglecting the influence of lithophysal porosity on heat capacity.

\subsubsection{Sensitivity of TH to design and operational parameters (MSTH)}

The analyses presented in this report focus on three goals: incorporating new science, quantifying uncertainties, and evaluating the performance and uncertainty associated with HTOM and LTOM. This section discusses the sensitivity of TH parameters to design and operating parameters that could be used to achieve a LTOM goal.

Figure 43 depicts the HTOM and LTOM in terms of values of peak waste package temperature resulting from different choices of design and operating parameters. The figure shows that the HTOM has waste package peak temperatures of about $175^{\circ} \mathrm{C}$, lower than the peak values presented in the Viability Assessment of a Repository at Yucca Mountain, Total System Performance Assessment (DOE 1998 [DIRS 100550], Volume 3, Figure 3-22), which shows a peak temperature of about $200^{\circ} \mathrm{C}$ using bin-averaged, rather than hottest waste package, temperatures. Two LTOM options are shown, both resulting in peak waste package temperatures of about $85^{\circ} \mathrm{C}$ based on two-dimensional calculations.

Three design parameters- - drift diameter, drift spacing, and waste package capacity-are shown in the inner portion of Figure 43. These parameters were held constant for the purposes of this report, which focuses on determining performance and associated uncertainty for a range of thermal operating environments. If the site is recommended, design parameters can be varied during the preparation of a license application to optimize a combination of criteria, including worker safety, cost, and reduction in performance uncertainty.

The outer portion of Figure 43 shows operating parameters, including waste package spacing, forced and natural ventilation rates, and ventilation duration. These parameters can be changed even after a repository has been constructed. The two LTOM options use different values of operational parameters to approach the same TH conditions. The option analyzed in this document uses the first 64 drifts in the footprint (a contiguous, planar area), variable gaps between waste packages $\left(1.1-\mathrm{m}\right.$ average), and 300 years of forced ventilation at a rate of $15 \mathrm{~m}^{3} / \mathrm{s}$. The resulting linear heat loading at emplacement of this option is $1.13 \mathrm{~kW} / \mathrm{m}$ (Buscheck $2001 \mathrm{c}$ [DIRS 155449]).

Another lower-temperature option uses a larger footprint, has wider gaps between waste packages (2-m average), and shifts from forced to natural ventilation after 50 years (BSC 2001f [DIRS 155010]; BSC 20011 [DIRS 155011]). The natural ventilation rate used in the second reference is an average of $3 \mathrm{~m}^{3} / \mathrm{s}$ for the 50 - to 100 -year period and $1.5 \mathrm{~m}^{3} / \mathrm{s}$ for the 100 - to 300 -year period. The resulting linear heat loading at emplacement of this option is $1.0 \mathrm{~kW} / \mathrm{m}$. The two options trade forced ventilation duration and waste package spacing (and repository footprint).

Natural ventilation and other methods to achieve lower repository temperatures have been the subject of study for several years (BSC 2001k [DIRS 154855]; CRWMS M\&O 2000c [DIRS 152269]; CRWMS M\&O 2000e [DIRS 152146]). The lower-temperature option shown in (Figure 43) is based on design and operational parameters developed from sensitivity 
calculations in these three references. Additional documentation is in BSC (2001f [DIRS 155010]) and BSC (20011 [DIRS 155011]).

The in-drift TH parameter histories, including variability across the repository footprint, are shown in Section 5.4.2 for the LTOM. The MSTH model calculations (Buscheck 2001c [DIRS 155449]) were repeated for the same AML and linear heat loading of $1.13 \mathrm{~kW} / \mathrm{m}$ as the LTOM base case. The new (Buscheck 2001c [DIRS 155449]) used a line loading arrangement with $10-\mathrm{cm}$ gaps between all waste packages, rather than more widely spaced waste packages. The linear heat loading was maintained at the same level, since the waste packages were moved together by de-rating the hotter waste packages. The de-rating was done simplistically, by removing spent nuclear fuel assemblies from the hotter PWR waste packages until the target heat loading was achieved (at the 16 fuel assembly level). If a lower-capacity waste package option is developed during a potential license application, it could achieve goals of lower peak power by using smaller PWR waste packages or by blending BWR and PWR assemblies in the same waste package. Flexibility could be reserved for the blending of the two types of assemblies by developing an adapter-insert that would fit into the PWR basket slot and contain an opening the size of the smaller BWR assemblies.

Figure 44 compares the postclosure distribution of peak waste package temperature and relative humidity across the repository footprint for the LTOM option described in this report and the de-rated waste package capacity option for the medium infiltration history case, although the MSTH model results also include cases for lower and higher infiltration levels. The distributions are displayed as cumulative complementary distribution functions; the values on the $y$-axis are the fraction of waste packages hotter than the indicated value on the $\mathrm{x}$-axis. The base case resulted in 98.3 percent of the waste packages not exceeding the goal of $85^{\circ} \mathrm{C}$, and a peak of $86.0^{\circ} \mathrm{C}$ for the hottest waste package in the 7620 calculated waste package temperature-time histories. The abstracted temperature histories used in TSPA are somewhat lower because histories are grouped into bins, within which the average is used. The de-rated waste package case resulted in 100 percent of the waste packages not exceeding the goal of $85^{\circ} \mathrm{C}$, and a peak of $82.6^{\circ} \mathrm{C}$ for the hottest waste package. The de-rated waste package option had slightly cooler results for the same repository footprint. The cooler temperatures are due to the smaller range of thermal powers among the waste packages and the more effective radiation heat transfer between closely spaced waste packages.

For waste packages that exceed the $85^{\circ} \mathrm{C}$ goal, a low humidity can prevent the formation of aqueous films that are required for corrosion initiation. Figure 44 also shows the postclosure relative humidity distribution (among the 1.7 percent of the ensemble of waste packages that exceed $85^{\circ} \mathrm{C}$ for some period of time in the LTOM base case) when the temperature of each waste package falls below $85^{\circ} \mathrm{C}$. The initiation threshold for crevice corrosion of $85^{\circ} \mathrm{C}$ is based on more aggressive water chemistry than is expected on waste package surfaces.

To compare other options, a simplified version of the MSTH model was used (Buscheck 2001c [DIRS 155449]). In the simpler model, the same four submodels were used, but only a single location (15c3) was used for the smeared-heat-source drift-scale temperature (SDT) and line-source drift-scale thermal-hydrologic submodels. The $15 \mathrm{c} 3$ location is near the repository center and is within the lower-lithophysal stratigraphic unit. 
Figure 45 shows postclosure temperature and relative humidity histories (Buscheck 2001c [DIRS 155449]) for the hottest waste package surface for three LTOM implementations that share the same areal mass loading of 45.7 MTU/acre: the LTOM base case, the de-rated waste package case, and a wider drift spacing case that uses the HTOM waste package arrangement, but with a drift spacing of $97 \mathrm{~m}$ instead of $81 \mathrm{~m}$. As summarized in Table 25, the peak temperatures are $84.3^{\circ} \mathrm{C}$ for the base case, $81.7^{\circ} \mathrm{C}$ for the de-rated waste package case, and $88.8^{\circ} \mathrm{C}$ for the wider drift spacing case. These results indicate that all three methods achieve similar temperatures for LTOM designs. Line loading with a limited range of waste package thermal powers (i.e., using de-rated waste packages) is most effective at limiting peak temperature, and wider spacing of waste packages within the drifts is more effective than wider spacing of drifts. The relative effectiveness of these design and operating parameters (waste package capacity, drift spacing, and waste package spacing) is consistent with the conceptual understanding of three-dimensional radiation heat transfer among the waste packages.

A comparison of Figure 44 and Figure 45 shows that the simplified MSTH model calculates peak temperatures about 1 to $2^{\circ} \mathrm{C}$ less than the full MSTH model. It was expected that the peak temperatures would be somewhat lower because it is not likely that the location chosen for the simplified MSTH model would be the location of peak power. The location was chosen, however, to be in a central region with low infiltration flux so that it would be near the overall peak temperature. The advantage of using the simplified MSTH model is that additional design variables can be investigated with a smaller suite of calculations, yet with results (at the chosen location) that are the same as the full MSTH model results.

The postclosure relative humidity histories at the hottest waste package surface are shown in Figure 45 for the three LTOM cases. Depending on the composition of the dust on the waste package surface, the threshold relative humidity for water film formation (deliquescence) varies. Figure 45 can be used to determine the time at which the threshold humidity is reached. This value can then be used with the temperature panel of the same figure to estimate the likelihood of crevice corrosion during the thermal pulse for the hottest waste packages in the repository. The figures indicate that only the case with wider drift spacing is above the $85^{\circ} \mathrm{C}$ temperature threshold., During the time that the surfaces of some waste packages are above $85^{\circ} \mathrm{C}$, the humidity ranges between 48 and 69 percent for the wider drift spacing case. The maximum duration during which temperatures are above $85^{\circ} \mathrm{C}$ is about 500 years for that case. Figure $44 \mathrm{~b}$ shows that for the 1.7 percent of the waste packages that exceed $85^{\circ} \mathrm{C}$ for the LTOM-PA case, the relative humidity ranges between 48 and 62 percent during the time that the waste-package surface temperature exceeds $85^{\circ} \mathrm{C}$. Figure $44 \mathrm{~b}$ also shows that none of the waste packages exceeds $85^{\circ} \mathrm{C}$ for the de-rated waste-package case.[

\subsubsection{Sensitivity of Pre-closure Dryout (LDTH)}

Note that for the thermal-hydrologic model calculations conducted for this section, the ambient liquid saturation is 0.94 in the matrix continuum). In this section, we investigate whether explicitly accounting for rock dryout during the preclosure period significantly influences predicted thermal-hydrologic conditions during the postclosure period. The LDTH submodel at the $15 \mathrm{c} 3$ location was used to investigate this sensitivity. The location and infiltration boundary conditions for the two-dimensional submodel were the same as those described in Section 5.3.2.4.2. Four different cases (called Cases 1, 2, 3, and 4) are considered for both the 
HTOM and LTOM, with each case having different assumptions about thermal-hydrologic conditions within the emplacement drifts during the preclosure ventilation period. Note that for the thermal-hydrologic model calculations conducted for this section, the ambient liquid saturation is 0.94 in the matrix continuum).

For each of the four cases and for both of the operating modes, the model was implemented in three steps. The first step was to run the model during the preclosure period to obtain the drift-wall temperature history, using the base-case ventilation efficiencies: 70 percent for 50 years for the higher-temperature operating mode and 80 percent for 300 years for the lower-temperature operating mode. The second step was to repeat the preclosure calculation for the rock portion of the model, using the Step-One drift-wall temperature history and an assigned gas boundary condition at the drift wall.

In Step Two, the gas boundary condition was assigned to have a gas-phase pressure of 99 percent of ambient pressure and a relative humidity of 30 percent during the preclosure ventilation period for Case 1. A relative humidity of 30 percent is close to the mean annual relative humidity at the ground surface at Yucca Mountain. The psychrometric properties of the intake air for the ventilation of the emplacement drifts are summarized in (BSC 2001a [DIRS 155246], Table XXVII-1). The emplacement drifts are ventilated by pulling (rather than pushing) air, which causes the gas-phase pressure in the ventilated drifts to be reduced by a maximum of about 1 percent relative to ambient conditions (CRWMS M\&O 2000h [DIRS 154176], p. 22). Cases 2 and 3 assign different values of either gas-phase pressure or relative humidity, while Case 4 uses different ventilation efficiencies than in Cases 1, 2, and 3. Thus, the ventilated emplacement drift functions as a sink for the humid gas in the host rock due to the lower gasphase pressure and lower water vapor content of the in-drift air compared to the air in the near-field rock. Note that under ambient conditions, relative humidity in the host rock is greater than 99 percent.

The final preclosure distributions of temperature, liquid saturation, gas-phase pressure, and relative humidity in the host rock from Step Two become the initial rock conditions for the StepThree postclosure calculation. Thus, Step Three captures the influence of preclosure dryout of the host rock. Step Three is similar to Step One in that the line-averaged heat-generation rate of the waste packages is represented in the LDTH submodel, rather than representing the influence of this heat-generation-rate with a specified boundary temperature as was done in Step Two. During the postclosure period, 100 percent of the heat generated by waste packages is represented in the LDTH model. Figure 46 and Figure 47 show the heat-generation histories for the higher- and lower-operating modes, respectively.

This three-step process was repeated for Cases 2, 3, and 4 for both the higher- and lower-temperature modes. Case 2 is the same as Case 1 except relative humidity in the drift is assigned to be 5 percent (instead of 30 percent) during the preclosure period. The value of 5 percent corresponds to the minimum relative humidity of the ventilation air ( 4.77 percent), which is calculated to occur at 10 years (BSC 2001a [DIRS 155246]). A relative humidity of 5 percent represents an extreme lower bound for the in-drift relative humidity conditions during the ventilation period. Case 3 is the same as Case 1 except gas-phase pressure in the drift is assigned to be 95 percent of ambient during the preclosure period. Case 4 is the same as Case 1 except the ventilation efficiencies are assigned to be 60 percent for 50 years for the HTOM and 
70 percent for 300 years for the LTOM (10 percent less than the other cases for both operating modes).

Figure 48 shows the lateral extent of host-rock dryout at the end of the preclosure ventilation period for Case 1. At the time of closure, liquid saturation in the matrix is reduced to about 0.5 at distances of $3 \mathrm{~m}$ and $1 \mathrm{~m}$ laterally away from the drift wall, for the higher- and lower-temperature operating modes, respectively.

For the higher-temperature operating mode, Figure 49 compares the results of Case 1 to those of the base-case 15c3 LDTH submodel (the base case neglects the influence of rock dryout during the preclosure period). The results of the base-case 15c3 LDTH submodel, together with those of the LDTH submodels at the 32 other geographic locations in the repository, are included in the generation of the MSTH model results described in Section 6.2.1. Figure 49 shows that Case 1 and the base case result in almost identical postclosure temperature at the drift wall and drip shield, relative humidity at the drip shield, and liquid saturation in the invert and at the drift wall. The preclosure dryout predicted for Case 1 has a negligible effect on postclosure thermalhydrologic conditions around the drift. The rapid increase in temperatures (above the boiling point of water) following the cessation of ventilation generates a much larger dryout zone than that generated during the preclosure ventilation period, thereby overwhelming the influence of the preclosure dryout calculated in Step Two of the three-step calculation.

For the lower-temperature-operating mode, Figure 50 compares the results of Case 1 to those of the base-case 15c3 LDTH submodel, (the base case neglects the influence of rock dryout during the preclosure period). The results of the base-case 15c3 LDTH submodel, together with those of the LDTH submodels at the 32 other geographic locations in the repository, are included in the generation of the MSTH model results described in Section 6.2.2. Figure 50 shows that Case 1 and the base case result in almost identical postclosure temperature at the drift wall and drip shield, relative humidity at the drip shield, and liquid saturation in the invert and at the drift wall. The preclosure dryout predicted for Case 1 has a negligible effect on postclosure thermalhydrologic conditions around the drift. Rewetting of the host rock (primarily by matrix imbibition) requires about one hundred years after closure. However, peak temperatures at the drift wall and drip shield occur even later, two to three hundred years after closure for the lower-temperature operating mode, and are not affected by the inclusion of preclosure dryout in the LDTH-submodel calculation. Because the dryout zone (which corresponds to the region of reduced thermal conductivity in the host rock) has collapsed prior to the occurrence of peak temperatures for the LTOM, rock dryout has a negligible influence on peak temperatures, as well as on other thermal-hydrologic conditions in the emplacement drifts.

Figure 51 compares the lateral extent of host-rock dryout at the end of the preclosure ventilation period for the HTOM and LTOM for Cases 1, 2, 3, and 4. The lower input value of relative humidity in the drift for Case 2 ( 5 percent) compared to Case 1 ( 30 percent) causes the lateral extent of dryout to extend further into the rock. The lower input value of gas-phase pressure in the drift for Case 3 ( 95 percent of ambient) compared to Case 1 ( 99 percent of ambient) causes the lateral extent of dryout to extend further into the rock. Case 4 , which uses a lower ventilation efficiency than Case 1, results in higher temperature at the drift wall during the preclosure ventilation period and causes the lateral extent of dryout to extend further into the rock. For all three cases (2-4), the influence of this effect is greater for the higher-temperature-operating mode 
than for the lower-temperature operating mode. Summarizing, the lateral extent of host-rock dryout increases with decreasing relative humidity and gas-phase pressure in the drift and it increases with increasing drift-wall temperature. Figure 52 compares Cases 1, 2, 3, and 4 for the HTOM. Although Cases 1, 2, and 3 produced slightly different lateral extents of preclosure dryout, they result in almost identical postclosure temperature at the drift wall and drip shield, relative humidity at the drip shield, and liquid saturation in the invert and at the drift wall. Recall that Case 1 has a virtually identical temperature, liquid saturation, and relative humidity conditions as in the base-case LDTH submodel that ignored the influence of preclosure dryout. Therefore, Cases 1, 2, and 3 result in the same temperature, liquid saturation, and relative humidity conditions as in the base-case LDTH submodel that neglects preclosure dryout. Case 4 results in slightly higher temperatures, and in slightly lower liquid saturation and relative humidity, than in Cases 1, 2, and 3. The primary cause of the higher temperature and lower liquid saturation and relative humidity in Case 4 is that it utilized a lower heat removal efficiency during the preclosure ventilation period, resulting in higher temperatures at the beginning of the postclosure period. The secondary cause of the higher temperatures is the large dryout zone that results in a larger region of reduced thermal conductivity, causing postclosure temperatures to be somewhat higher.

Figure 53 compares Cases 1, 2, 3, and 4 for the lower-temperature-operating mode. Cases 1, 2, and 3 produce essentially identical postclosure temperature at the drift wall and drip shield, relative humidity at the drip shield, and liquid saturation in the invert and at the drift wall. Recall that Case 1 has similar temperature, liquid saturation, and relative humidity conditions as in the base-case LDTH submodel that ignored the influence of preclosure dryout. Therefore, Cases 1 , 2 , and 3 result in the same temperature, liquid saturation, and relative humidity conditions as in the base-case LDTH submodel that neglects preclosure dryout. Case 4 also results in similar liquid saturation and relative humidity conditions; however, Case 4 results in slightly higher temperatures than in the other cases. The cause of the higher temperature in Case 4 is that it utilized a lower heat removal efficiency during the preclosure ventilation period, resulting in higher temperatures at the beginning of the postclosure period.

\subsubsection{Sensitivity of 3D In-Drift Effects (DDT)}

The variations in geometry and thermal output of waste package will result in variation of temperature within a cross-section of an emplacement drift and along the drift axis. Because the in-drift air will be circulated due to natural convection, resulting in a spatially uniform composition, water will preferentially evaporate from warmer surfaces and condense on cooler surfaces due to the small difference in equilibrium of vapor pressure at the temperatures of the surfaces. Surface roughness and the presence of dust will influence the vapor pressure at the surfaces.

The MSTH model (CRWMS M\&O 2000b [DIRS 149862]) includes evaporation and condensation on in-drift surfaces, based on thermodynamic properties of the gas and liquid phases. The water then moves along the drift surface under the influence of gravity and capillary forces in the highly permeable porous medium used to simulate the air. This treatment captures much of the two-phase process; however, it does not calculate the details of the geometry of condensation (film thickness or growth of individual drops) on surfaces which can be affected by roughness and on dust which can dissolve into the condensing water. 
The TSPA-SR (CRWMS M\&O 2000j [DIRS 153246]) model includes humid-air corrosion of the waste packages and drip shields when in-drift air humidity is high enough to deliquesce onto salts in the dust. Therefore, condensation on the waste packages and drip shields is assumed in the current implementation of corrosion in TSPA. In TSPA-SR (CRWMS M\&O 2000j [DIRS 153246]), radionuclide transport in the engineered barrier system was assumed to be diffusive until breach of the drip shields by corrosion or disruptive events; the basis of this assumption was that condensate film or drops could be too thin (or small) to flow, flow very slowly, or be hindered by corrosion products, detrital material, or mineral precipitates.

For one SSPA Volume 2 (McNeish 2001 [DIRS 155023]) unquantified uncertainty sensitivity study, the condensation model was improved. Condensation on the underside of the drip shield can occur in the improved model if the vapor pressure at the invert is higher than the saturation pressure at the drip shield. This situation is implemented by assuming there is condensation when the drip shield temperature is less than the invert temperature; the implementation ignores chemical and capillary effects on the vapor pressure in the invert. Condensation is assumed to drip from the drip shield onto the waste package, analogous to the dripping of seepage from a drip shield breach. The volume of condensation is sampled from zero to 100 percent of the evaporation rate of water from the invert (an output of the MSTH model). This sampling qualitatively accounts for the competition for evaporated water by other cool surfaces such as the drift wall and for run-off of some of the condensate along the side of the drip shield.

Improvements in the three-dimensional DDT submodel of the MSTH model have increased the accuracy of in-drift temperature history calculations that support the improved condensation model described in Section 8.3.2 of SSPA Volume 2 (McNeish 2001 [DIRS 155023]). Results of the base case calculations (Buscheck 2001b [DIRS 155243]) for the HTOM and LTOM are summarized in Table 26 and 6.1.8-2, respectively. Those results are the focus of the discussion following the tables.

In addition to being an input to the improved, but simple, condensation model in Section 8.3 .2 of SSPA Volume 2 (McNeish 2001 [DIRS 155023]), the in-drift temperature histories could be used in detailed condensate geometry calculations in support of a potential license application. Section 10.3 includes a discussion of models to determine condensation film thickness as a function of substrate material, temperature, and relative humidity, to estimate the area for diffusion of radionuclides within the film. That analysis could be useful in developing a model to determine if condensation could form a source for slow flow that could support advection. Test results from the quarter-scale canister tests (Howard et al. 2001 [DIRS 153282]) and from the scope of work that is identified in the technical work plan for the sealed section of the ECRB cross-drift (BSC 2001i [DIRS 155051], Section 1, Items 27 to 33) are other inputs that can be used to support condensate formation and flow modeling.

Table 26 illustrates the temperatures in three cross-sections through the ten waste package computational cell for the HTOM. The cell includes ten full waste packages and two half waste packages, with the distribution of waste package types and thermal powers being representative of the full inventory of the repository. The three cross-sections are through the mid-lengths of the hottest waste package (pressurized water reactor, PWR), an average power waste package (boiling water reactor, BWR), and a cool waste package (defense high level waste DHLW). The times selected include the time of peak preclosure temperature, just after closure, peak 
postclosure temperature, three times during the slow cooling of the repository (as the waste decays), and a final time at which near-ambient temperatures have been reached. The DDT submodel represents radiation under and above the drip shield explicitly, but uses a perimeter-averaged correlation to represent natural convection. More accurate temperatures along the drift wall perimeter will be available when Navier-Stokes computational fluid dynamics models are applied to this system.

During the preclosure period, the low relative humidity of the ventilation air will limit the water content of the in-drift air enough that water films will be negligible. At the peak of the postclosure temperature for the HTOM, all temperatures are sufficiently high that water films would not form. However, under some conditions, highly deliquescent salts in dust on some components may cause condensate formation at low humidity or elevated temperature; these conditions are being studied in support of a potential license application.

Two thousand years after the waste emplacement, before temperatures have cooled to below-boiling values, the range of temperature differences on all surfaces within the three-dimensional cell is less than $4^{\circ} \mathrm{C}$. Within a cross section, the range is as little as several tenths of a degree at the cool waste package to as much as almost $3^{\circ} \mathrm{C}$ at the design basis (hottest) waste package. Along the drift axis, the range is less than $1{ }^{\circ} \mathrm{C}$ at the drift wall, and as much as about $3^{\circ} \mathrm{C}$ at the waste package. Thus, the axial range is similar to the range within a cross section.

As temperatures decrease through the boiling point, the coolest surfaces in the drift are at the drift wall. The invert zone under the drip shield is slightly warmer than the drip shield itself. Since the invert temperature is an average through its half-depth, its surface is hotter than either its average or the drip shield surface. Thus, for the natural convection (perimeter-averaged) correlation and the low value of invert thermal conductivity used in the calculation, condensation would be favored on the drift wall near the coolest waste package. However, condensation depends on surface conditions as well as temperature; thus the temperature relationships are not enough to draw conclusions about condensate geometry on rock, titanium, and gravel.

Airflow patterns of natural convection are expected to shift the temperature of the drip shield (upper region) upward and the temperature of the invert surface under the drip shield downward. Therefore, natural convection could result in a cooler invert than the drip shield, reducing potential condensation on the underside of the drip shield. As corroboration of this logic, observations of the quarter-scale canister test were that the invert was cooler than the drip shield, with no dripping or rivulets observed under the drip shield (Howard et al. 2001 [DIRS 153282]).

Table 27 illustrates the temperatures in five cross-sections through the ten waste package computational cell for the LTOM. The cell includes the same sequence of waste packages as for the HTOM. However, there are variable length gaps between the waste packages with the goal of smoothing the local lineal heat loading. This geometric smoothing is an alternative to the axial radiation heat transfer between waste package ends that smoothes the local lineal heat loading in the HTOM. The five cross-sections are through the mid-lengths of the hottest waste package (PWR), an average power waste package (BWR), a cool waste package (DHLW), a warm gap, and a cool gap between waste packages. The times selected include the time of peak preclosure temperature, just after closure, three times representing the broad period of near-peak 
postclosure temperature, during the slow cooling of the repository (as the waste decays), and a final time at which near-ambient temperatures have been reached.

During the preclosure period, the low relative humidity of the ventilation air will limit the water content of the in-drift air enough that water films will be negligible. Under some conditions, highly deliquescent salts in dust on some components may be able to cause condensation at low humidity; these conditions are being studied in support of a potential license application. As soon as ventilation ceases for the LTOM, all temperatures are sufficiently low that condensate could form.

At the time of peak waste package temperature, the range of temperature differences on all surfaces within the three-dimensional cell is about $10^{\circ} \mathrm{C}$. Within a cross section, the range is as little as $1^{\circ} \mathrm{C}$ at the cool waste package to as much as $8^{\circ} \mathrm{C}$ at the design basis (hottest) waste package. Along the drift axis, the range is about $2^{\circ} \mathrm{C}$ at the drift wall, and about $9^{\circ} \mathrm{C}$ at the waste package. Thus, the axial range is similar to the range within a cross section.

As temperatures cool, the coolest surfaces in the drift are at the drift wall. The invert zone under the drip shield is slightly warmer than the drip shield itself. Because the invert temperature is an average through its half-depth, the surface is hotter than either the average or the drip shield surface. As for the HTOM, shifts in temperature patterns around the perimeter of air flow loops could reverse the temperature relationship between the drip shield and invert surface, resulting in little condensation on the drip shield underside.

\subsection{MSTH Calculations in Support of SSPA, Vol.2 (TSPA Parameters)}

\subsubsection{HTOM Results}

This section describes the MSTH model base-case calculations (Buscheck 2001b [DIRS 155243]) for the HTOM, which includes the mean, upper, and lower infiltration-flux scenarios. Figure 54 is a plan view of the MSTH model representation of the potential HTOM repository; also shown are the geographic locations for which $\mathrm{TH}$ conditions are shown in Figure 55 and Figure 56. These locations were chosen to illustrate the manner in which TH behavior is influenced by proximity to the edges of the repository. Three of these locations are close to the center of the repository; two are located in the extreme northeast and southwest corners of the repository area, and two are at the extreme eastern and western edges of the repository, approximately midway between the northern and southern boundaries of the repository. The temperature and relative-humidity histories fall into three distinct clusters: one for the repository center, one for the repository corners, and one for the repository edge locations (Figure 55). In addition, the relative humidity reduction can be classified into three sequential periods (Figure 55).

During an early period which lasts from 200 to 1,000 yr (Figure 55c and Figure 56a), the relative humidity reduction on waste packages depends on the magnitude and duration of rock dryout. The duration of rock-dryout increases with distance from the repository edges. The farther a given waste package is located from the repository edge, the longer the duration of boiling in the local host rock and the longer the duration of rock dryout (and relative humidity reduction). The surface of the waste packages always have a lower relative humidity than the adjacent host rock; 
consequently, during the early period, the reduction in relative humidity on waste packages increases with distance from the repository edges.

The depth of dryout varies with location within the footprint and with infiltration flux. The deepest horizontal extent of boiling temperatures was 13.1, 10.6, and $10.1 \mathrm{~m}$ from the drift center, for the lower, mean, and upper infiltration flux scenarios, respectively.

An intermediate period, which lasts from $1,000 \mathrm{yr}$ to about $20,000 \mathrm{yr}$, during which time heat-transfer in the drift controls the magnitude of relative humidity reduction on waste packages. During this period, the reduction in relative humidity on waste packages decreases with distance from the repository edges, which is the reverse of the trend during the early period. During the intermediate period, the reduction in relative humidity is greater at the repository edges because the efficiency of thermal radiative heat transfer decreases with temperature. Consequently, for a given local heat generation rate there is a larger temperature difference $\Delta T_{\text {wp-dw }}$ between the waste package and drift wall at locations with lower temperature (such as occurs close to the repository edges). Moreover, the reduction in relative humidity, which depends on the ratio of $P_{\mathrm{sat}}\left(T_{\mathrm{dw}}\right) / P_{\mathrm{sat}}\left(T_{\mathrm{wp}}\right)$, results in a larger relative humidity reduction for a given $\Delta T_{\mathrm{wp}-\mathrm{dw}}$, where $P_{\text {sat }}$ is the saturation vapor pressure, $T_{\mathrm{dw}}$ is the drift wall temperature, and $T_{\text {wp }}$ is the waste package temperature.

A late period, beginning around $20,000 \mathrm{yr}$, during which time the reduction in relative humidity on waste packages becomes decreasingly sensitive to proximity to the repository edges. For a given waste package type, the reduction in relative humidity becomes increasingly similar across the repository area.

Figure 57 shows the influence of the edge-cooling effect. Locations close to the repository edges cooling more quickly than those at the center. Figure 58 shows the development of the reduction in relative humidity on waste packages. At early times, the reduction in relative humidity increases with distance away from the repository edges, while at intermediate to later times, the reduction in relative humidity decreases with distance from the repository edges.

Figure 56b and Figure 56c show the relationship between liquid saturation in the invert and evaporation rate. During early times, while the invert is dry, the evaporation rate is zero. The onset of rewetting in the invert corresponds to the end of the boiling/rock-dryout period. Consequently, the time of the onset of rewetting increases with distance from the repository edges. The two geographic locations that experience a larger eventual increase in liquid saturation in the invert correspond to the two locations wherein the local host rock is either the middle or the lower nonlithophysal Topopah Spring welded tuff unit (Tptpmn or Tptpln). Wherever the local host-rock unit is comprised of the lower lithophysal unit (Tptpll), the maximum liquid saturation in the invert is nearly the same (and lower than in locations where the local host-rock unit is either Tptpmn or Tptpln). Apparently, there is a difference in rewetting behavior in the invert that depends on the local host-rock unit. The second spike in the invert evaporation rate occurs shortly after 600 years when a substantial increase in infiltration flux occurs. The increase in infiltration rate is accompanied by an increase in liquid-phase flux into the invert, which makes more water available for evaporation in the invert. 
Figure 59 shows the complementary cumulative distribution functions for temperature and relative humidity on the drift wall and on the waste package for the mean, lower, and upper infiltration-flux scenarios. For the mean-infiltration-flux scenario, the peak waste package temperature ranges from $126.1^{\circ}$ to $184.9^{\circ} \mathrm{C}$. The peak waste package temperature is similar for the mean and lower infiltration-flux scenarios. The upper infiltration-flux scenarios result in lower peak waste package temperatures, particularly for the coolest waste packages; the range for this scenario is $105.4^{\circ}$ to $183.1^{\circ} \mathrm{C}$. The time required for the drift-wall to cool down to $96^{\circ} \mathrm{C}$ (which is the boiling point of water at the repository horizon) is more sensitive to infiltration flux than peak temperature (Figure $59 \mathrm{~b}$ ). The time required for the waste package to attain relative humidity equal to 80 percent is similar for the mean and upper-infiltration flux scenarios (Figure 59c). The lower-infiltration-flux scenario results in much more persistent relative humidity reduction on waste packages, particularly for the driest waste packages. The waste package temperature at the time when relative humidity equals 80 percent on the waste package is similar for the mean and upper infiltration-flux scenarios (Figure 59d); the lower infiltration-flux scenario results in lower temperatures when relative humidity equals 80 percent is attained.

Figure 60 shows the waste package-to-waste package variability of temperature and relative humidity at a location in the repository relatively close to the geographic center for the mean infiltration-flux scenario. Peak waste package temperatures range from $152.3^{\circ}$ to $180.1^{\circ} \mathrm{C}$. The use of line-load waste package spacing results in a relatively narrow range of peak temperatures $\left(27.8^{\circ} \mathrm{C}\right)$. The three coolest waste packages are all DHLW waste packages. The three hottest waste packages are all PWR waste packages. The old PWR waste package and BWR waste packages fall in the middle of the temperature range. The reduction in relative humidity on waste packages correlates directly with temperature on the waste package. Therefore the PWR waste packages always experience the greatest relative humidity reduction, while the DHLW waste packages experience the least relative humidity reduction. At late times there is a greater range of relative humidity reduction resulting from waste package-to-waste package variability than arising from the distance from the repository edges (compare Figure 60b and Figure 55d).

The HTOM thermal-hydrologic results were abstracted and provided to TSPA (Francis and Itamura 2001 [DIRS 155321]) for use in SSPA Volume 2 (McNeish 2001 [DIRS 155023]).

\subsubsection{LTOM Results}

This section describes the multiscale thermal-hydrologic (MSTH) model base-case calculations (Buscheck 2001c [DIRS 155449]) for the LTOM, which includes the mean, upper, and lower infiltration-flux scenarios. Figure 61 gives the plan view of the MSTH model representation of the potential LTOM repository; also shown are the geographic locations for which TH conditions are given in Figure 62 and Figure 63. The potential LTOM repository area is 20 percent larger than the potential HTOM repository (Source: Produced using files from Buscheck 2001 [DIRS 155449].

NOTE:Plan view of the potential Higher-Temperature Operating Mode repository showing the geographic locations considered in various analyses. $\mathrm{X}=$ east-west distances, $\mathrm{Y}=$ north-south distances. 
Figure 54). These locations were chosen to illustrate the manner in which TH behavior is influenced by proximity to the edges of the potential repository. Four of these locations are close to the center of the potential repository; two are located in the extreme northeast and southwest corners of the potential repository area, and two are at the extreme eastern and western edges of the area, approximately midway between the northern and southern boundaries. The temperature and relative-humidity histories fall into three distinct clusters (one for the potential repository-center locations, one for the potential repository corners, and one for the potential repository-edge locations), and the reduction in relative humidity can be classified into two sequential periods (Figure 62).

An early-to-intermediate period lasts for about 20,000 years, during which time heat-transfer in the drift controls the magnitude of the reduction in relative humidity on waste packages. Because the temperatures for the LTOM are always below the boiling point of water, there is no rock dryout period, and no reduction in relative humidity in the host rock (Figure 62c); therefore, all reductions in relative humidity on waste packages arise as a result of the temperature differences, $\Delta T_{\text {wp-dw }}$, between waste packages and the drift wall. The early-to-intermediate period for the LTOM case has the same trends as observed for the intermediate period for the HTOM case (Section 6.2.1). During this period, the reduction in relative humidity on waste packages decreases with distance from the repository edges.

A late period, beginning around 20,000 years, occurs during which the reduction in relative humidity on waste packages becomes decreasingly sensitive to proximity to the repository edges. For a given waste-package type, the reduction in relative humidity becomes increasingly similar across the repository area. Figure 64 shows the influence of the edge-cooling effect, with locations close to the potential repository edges cooling more quickly than those at the center. Figure 65 also shows the trend of the reduction in relative humidity on waste packages increasing with proximity to the repository edges.

Figure $63 \mathrm{a}$ and Figure $63 \mathrm{~b}$ show the relationship between liquid saturation in the invert and evaporation rate. At the end of the ventilation period, the invert is assumed to be dry in the model. Rewetting of the invert begins immediately after the end of the ventilation period, which occurs at $300 \mathrm{yr}$. Therefore, evaporation rates in the invert are always nonzero. Rewetting of the invert occurs more quickly at repository-edge locations than at repository-center locations because the edge-cooling effect reduces the amount of heating available to evaporate the incoming water. At $300 \mathrm{yr}$, a rapid increase is seen in the evaporation rate as the liquid-phase flux into the invert immediately increases following the end of the ventilation period. The two geographic locations that experience a larger eventual increase in liquid saturation in the invert correspond to the two locations wherein the local host rock is either the middle or lower non-lithophysal Topopah Spring welded tuff unit (Tptpmn or Tptpln). Wherever the local host-rock unit is comprised of the lower lithophysal unit (Tptpll), the maximum liquid saturation in the invert is nearly the same (and lower than in locations where the local host-rock unit is Tptpmn or Tptpln). Apparently, there is a difference in re-wetting behavior in the invert that depends on the local host-rock unit.

Figure 66 gives the complementary cumulative distribution functions for temperature and relative humidity on the waste package for the mean, lower, and upper infiltration-flux scenarios. For the mean-infiltration-flux scenario, the peak waste package temperatures range from $59.2^{\circ}$ to 
$86.0^{\circ} \mathrm{C}$, with only 1.7 percent of the waste packages ever exceeding $85^{\circ} \mathrm{C}$. For the low-infiltration-flux scenario, the temperatures are higher than in the mean-infiltration-flux scenario, ranging from $60.5^{\circ}$ to $87.6^{\circ} \mathrm{C}$, with only 7.2 percent of the waste packages ever exceeding $85^{\circ} \mathrm{C}$. The upper infiltration-flux scenario results in lower peak waste package temperature that ranges from $59.0^{\circ}$ to $85.3^{\circ} \mathrm{C}$, with only 0.02 percent of the waste packages ever exceeding $85^{\circ} \mathrm{C}$.

Figure 66b, which gives the complementary cumulative distribution function of the time required for waste packages to attain a relative humidity of 80 percent show a distinct change in the slope of the curves at a complementary cumulative distribution function value of 0.7 ; this sharp break in the slope of the complementary cumulative distribution function curves indicates that there are two distinctively different groups of waste packages with respect to the duration of relatively dry relative humidity on the waste-package surfaces. In the MSTH model (Buscheck 2001c [DIRS 155449]), 30 percent of the waste packages are DHLW waste packages and 70 percent are CSNF waste packages. A detailed inspection of the underlying data that is plotted in Figure $66 \mathrm{~b}$ shows that the break in slope at a complementary cumulative distribution function value of 0.7 correspond with the fact that 30 percent of the waste-package inventory that attains an relative humidity of 80 percent first (i.e., having a complementary cumulative distribution function value between 0.7 and 1.0 ) is entirely comprised of DHLW waste packages. Those waste packages that have a complementary cumulative distribution function value between 0.0 and 0.7 are nearly entirely comprised of CSNF waste packages. Thus, 99 percent of the DHLW waste packages have a relatively short duration of reduced relative humidity, requiring less than 877 years to attain a relative humidity of 80 percent; the remaining 1 percent of the DHLW waste packages require between 877 and 1020 years to attain a relative humidity of 30 percent. All CSNF (PWR and BWR) waste packages require at least 877 years to attain a relative humidity of 80 percent; the driest PWR requires 4864 years to attain a relative humidity of 80 percent.

The time required for the waste package to attain a relative humidity of 80 percent is similar for all three infiltration-flux scenarios (Figure 66b). Because rock dryout does not occur, the reduction in relative humidity depends entirely on multi-scale heat flow occurring in the rock at the mountain scale and within the drift at the waste package scale. The waste package temperature at the time when the relative humidity reaches 80 percent on the waste package is highest for the lower-infiltration-flux scenario and lowest for the upper infiltration-flux scenario (Figure 66c).

The LTOM results in a more persistent relative humidity reduction on waste packages than does the HTOM (compare Figure 66b with Figure 66c). Moreover, the LTOM results in lower waste package temperatures at 80 percent relative humidity than does the HTOM (compare Figure 66c with Figure 66d). In general, the LTOM case results in lower waste package temperatures at any given value of relative humidity on the waste package than does the HTOM case.

Figure 67 shows the waste package-to-waste package variability in temperature and relative humidity at a location in the potential repository that is relatively close to the geographic center. Peak waste package temperatures range from 73.1 to $85.4^{\circ} \mathrm{C}$. The three coolest waste packages are all DHLW waste packages. The three hottest waste packages are all PWR waste packages. The old PWR waste package and BWR waste packages fall in the middle of the temperature range. The reduction in relative humidity on waste packages correlates directly with temperature 
on the waste package. Therefore the PWR waste packages always experience the greatest reduction in relative humidity, while the DHLW waste packages experience the smallest reduction in relative humidity. At late times, there is a greater range of relative humidity reduction resulting from waste package-to-waste package variability than arising from the distance from the potential repository edges (compare Figure $67 \mathrm{~b}$ with Figure $62 \mathrm{~d}$ ).

Figure 68 compares the TH conditions on typical PWR waste package surfaces for the HTOM and LTOM cases. All geographic locations (edge, corner, and center) are included within the shaded regions for the two operating modes. The bands of the $\mathrm{TH}$ conditions represent the progression in time, ending at ambient conditions of about $25^{\circ} \mathrm{C}$ and 100 percent relative humidity. The LTOM results in lower relative humidity for any given temperature, or lower temperature for any given relative humidity for all locations except for the corner of the repository where the HTOM results in lower relative humidity or temperature than the central LTOM locations.

The LTOM thermal-hydrologic results were abstracted and provided to TSPA (Francis and Itamura 2001 [DIRS 155321]) for use in Volume 2 (McNeish 2001 [DIRS 155023]).

\section{REFERENCES}

\subsection{Documents Cited}

ASME (American Society of Mechanical Engineers) 1995. "Materials." Section II of 1995 ASME Boiler and Pressure Vessel Code. New York, New York: American Society of Mechanical Engineers. TIC: 245287.

Avallone, E.A. and Baumeister, T., III, eds. 1987. Marks' Standard Handbook for Mechanical Engineers. 9th Edition. New York, New York: McGraw-Hill. TIC: 206891.

Bolz, R.E. and Tuve, G.L. 1973. CRC Handbook of Tables for Applied Engineering Science. 2nd Edition. Boca Raton, Florida: CRC Press. TIC: 246862.

BSC (Bechtel SAIC Company) 2001a. ANSYS Calculations in Support of Natural Ventilation Parametric Study for SR. CAL-SVS-HV-000003 REV 00 ICN 01. Las Vegas, Nevada: Bechtel SAIC Company. ACC: MOL.20010613.0250, DIRS 155246.

BSC (Bechtel SAIC Company) 2001b. Bases for the Supplemental Science and Performance Analyses (SSPA) Vol I Calculations. Input Transmittal 00455.T. Las Vegas, Nevada: Bechtel SAIC Company. ACC: MOL.20010522.0196, DIRS 154864.

BSC (Bechtel SAIC Company) 2001c. Design Input for the Engineered Barrier System Environment and Barriers. Input Transmittal 00422.Ta. Las Vegas, Nevada: Bechtel SAIC Company. ACC: MOL.20010405.0196, DIRS 154461.

BSC (Bechtel SAIC Company) 2001. Draft Calculation CAL-EBS-MD-000016 REV 00B. Input Transmittal 00397.T. Las Vegas, Nevada: Bechtel SAIC Company. ACC: MOL.20010529.0189. [DIRS 155195]. 
BSC (Bechtel SAIC Company) 2001d. FY 01 Supplemental Science and Performance Analyses, Volume 1: Scientific Bases and Analyses. TDR-MGR-MD-000007 REV 00 ICN 01. Las Vegas, Nevada: Bechtel SAIC Company. ACC: MOL.20010801.0404; MOL.20010712.0062; MOL.20010815.0001, DIRS 155950.

BSC (Bechtel SAIC Company) 2001e. FY01 Supplemental Science and Performance Analyses, Volume 2: Performance Analyses. TDR-MGR-PA-000001 REV 00. Las Vegas, Nevada: Bechtel SAIC Company. ACC: MOL.20010724.0110, DIRS 154659.

BSC (Bechtel SAIC Company) 2001f. Lower-Temperature Subsurface Facility and Ventilation Concepts Analysis (Review Copy). Input Transmittal 00469.T. Las Vegas, Nevada: Bechtel SAIC Company. ACC: MOL.20010529.0186, DIRS 155010.

BSC (Bechtel SAIC Company) 2001g. Lower-Temperature Subsurface Layout and Ventilation Concepts. .ANL-WER-MD-000002 REV 00. Las Vegas, Nevada: Bechtel SAIC Company. ACC: MOL.20010718.0225, DIRS 154554.

BSC (Bechtel SAIC Company) 2001h. Site Recommendation Subsurface Layout. ANL-SFSMG-000001 REV 00 ICN 02. Las Vegas, Nevada: Bechtel SAIC Company. ACC: MOL.20010411.0131, DIRS 154477.

BSC (Bechtel SAIC Company) 2001i. Technical Work Plan for Unsaturated Zone (UZ) Flow and Transport Process Model Report. TWP-NBS-HS-000001 REV 01. Las Vegas, Nevada: Bechtel SAIC Company. ACC: MOL.20010404.0007, DIRS 155051.

BSC (Bechtel SAIC Company) 2001j. Thermal Conductivity Properties for the Tptpll and Tptpul. Input Transmittal 00472.Ta. Las Vegas, Nevada: Bechtel SAIC Company. ACC: MOL.20010716.0060, DIRS 155008.

BSC (Bechtel SAIC Company) 2001k. Thermal Hydrology EBS Design Sensitivity Analysis. CAL-EBS-HS-000003 REV 00 ICN 01. Las Vegas, Nevada: Bechtel SAIC Company. ACC: MOL.20010525.0080, DIRS 154855.

BSC (Bechtel SAIC Company) 20011. Thermal Management Analysis for Low Temperature Design (Check Copy). Input Transmittal 00470.T. Las Vegas, Nevada: Bechtel SAIC Company. ACC: MOL.20010529.0187, DIRS 155011.

Buscheck, T.A. 2001a. "Delivery of Input \& Output Files for Sensitivity Calculations in the SSPA Volume 1 Report, Chapter 5." Memorandum from T.A. Buscheck (BSC) to J.A. Blink, June 25, 2001, PROJ.06/01.065, with enclosures. ACC: MOL.20010625.0388.

Buscheck, T.A. 2001b. "Delivery of Input and Output Files MSTHAC V.6.4.2 and the DDT Models Used in the SSPA Volume 1 Report, Chapter 5." Memorandum from T.A. Buscheck (BSC) to J.A. Blink, June 27, 2001, PROJ.06/01.075, with enclosures. ACC: MOL.20010713.0050.

Buscheck, T.A. 2001c. "Delivery of Input and Output Files MSTHAC V.6.4.2 and the DDT Models Used in the SSPA Volume 1, ICN 01, Chapter 5 Report Superceding Previous 
Submission." Memorandum from T.A. Buscheck (BSC) to J.A. Blink, July 17, 2001, PROJ.07/01.007, with enclosures. ACC: MOL.20010726.0064.

CRWMS M\&O 1999a. ANSYS Calculations in Support of Enhanced Design Alternatives. B00000000-01717-0210-00074 REV 00. Las Vegas, Nevada: CRWMS M\&O. ACC: MOL.19990218.0240, DIRS 102843.

CRWMS M\&O 1999b. Enhanced Design Alternative (EDA) II Repository Estimated Waste Package Types and Quantities. Input Transmittal EBS-SR-99325.T. Las Vegas, Nevada: CRWMS M\&O. ACC: MOL.19991103.0236, DIRS 128028.

CRWMS M\&O 1999c. Natural Environment Data for Engineered Barrier System (EBS) Base Case. Input Transmittal EBS-NEP-99273.T. Las Vegas, Nevada: CRWMS M\&O. ACC: MOL.19990920.0108, DIRS 110177.

CRWMS M\&O 1999d. Request for Repository Subsurface Design Information to Support TSPA-SR. Input Transmittal PA-SSR-99218.Ta. Las Vegas, Nevada: CRWMS M\&O. ACC: MOL.19990901.0312, DIRS 105663.

CRWMS M\&O 1999e. Tabulated In-Drift Geometric and Thermal Properties Used in DriftScale Models. Development Plan TDP-EBS-HS-000002 REV 00. Las Vegas, Nevada: CRWMS M\&O. ACC: MOL.19990824.0147. DIRS 124630.

CRWMS M\&O 2000. Analysis of Infiltration Uncertainty. ANL-NBS-HS-000027 REV 00. Las Vegas, Nevada: CRWMS M\&O. ACC: MOL.20000525.0377. DIRS 143244.

CRWMS M\&O 2000a. Invert Effective Thermal Conductivity Calculation. CAL-WIS-TH000004 REV 00. Las Vegas, Nevada: CRWMS M\&O. ACC: MOL.20000317.0593, DIRS 142736.

CRWMS M\&O 2000b. Multiscale Thermohydrologic Model. ANL-EBS-MD-000049 REV 00 ICN 01. Las Vegas, Nevada: CRWMS M\&O. ACC: MOL.20001208.0062, DIRS 149862.

CRWMS M\&O 2000c. Natural Ventilation Study: Demonstration of Concept. TDR-SVS-SE000001 REV 00. Las Vegas, Nevada: CRWMS M\&O. ACC: MOL.20001201.0103, DIRS 152269.

CRWMS M\&O 2000d. Near Field Environment Process Model Report. TDR-NBS-MD000001 REV 00 ICN 03. Las Vegas, Nevada: CRWMS M\&O. ACC: MOL.20001121.0041, DIRS 153363.

CRWMS M\&O 2000e. Operating a Below-Boiling Repository: Demonstration of Concept. TDR-WIS-SE-000001 REV 00. Las Vegas, Nevada: CRWMS M\&O. ACC: MOL.20001005.0010, DIRS 152146.

CRWMS M\&O 2000f. Repository Subsurface Design Information to Support TSPA-SR. Input Transmittal PA-SSR-99218.Tc. Las Vegas, Nevada: CRWMS M\&O. ACC: MOL.20000424.0690, DIRS 149137. 
CRWMS M\&O 2000g. Subsurface Facility System Description Document. SDD-SFS-SE000001 REV 01. Las Vegas, Nevada: CRWMS M\&O. ACC: MOL.20000807.0078, DIRS 151467.

CRWMS M\&O 2000h. Subsurface Radon Calculations. CAL-SSM-NU-000003 REV 00. Las Vegas, Nevada: CRWMS M\&O. ACC: MOL.20001220.0016, DIRS 154176.

CRWMS M\&O 2000j. Total System Performance Assessment for the Site Recommendation. TDR-WIS-PA-000001 REV 00 ICN 01. Las Vegas, Nevada: CRWMS M\&O. ACC: MOL.20001220.0045, DIRS 153246.

CRWMS M\&O 2000k. Ventilation Model. ANL-EBS-MD-000030 REV 00. Las Vegas, Nevada: CRWMS M\&O. ACC: MOL.20000107.0330, DIRS 120903.

CRWMS M\&O 2001. Abstraction of NFE Drift Thermodynamic Environment and Percolation Flux. ANL-EBS-HS-000003 REV 00 ICN 02. Las Vegas, Nevada: CRWMS M\&O. ACC: MOL.20010221.0160, DIRS 154594.

DOE (U.S. Department of Energy) 1998. Total System Performance Assessment. Volume 3 of Viability Assessment of a Repository at Yucca Mountain. DOE/RW-0508. Washington, D.C.: U.S. Department of Energy, Office of Civilian Radioactive Waste Management. ACC: MOL.19981007.0030.

Ellenwood, F.O. and Mackey, C.O. 1962. Thermodynamic Charts, Steam, Water, Ammonia, "Freon-12," and Mixtures of Air and Water Vapor. 2nd Edition. New York, New York: John Wiley \& Sons. TIC: 249904.

Francis, N.D. 2001. "Documentation of Parameter Development and Sensitivity Studies for InDrift Parameters Reported in the SSPA Volume 1 Report." Memorandum from N.D. Francis (SNL) to RPC, May 22, 2001, with attachment. ACC: MOL.20010601.0217.

Francis, N.D. and Itamura, M.T. 2001. "TH Abstraction Results for the LTOM and HTOM Design Options." Correspondence from N.D. Francis (SNL) and M.T. Itamura (SNL) to RPC, May 31, 2001. ACC: MOL.20010629.0101.

Hadley, G.R. 1986. "Thermal Conductivity of Packed Metal Powders." International Journal of Heat and Mass Transfer, 29, (6), 909-920. [New York, New York]: Pergamon Journals. TIC: 249320.

Hahn, G.J. and Shapiro, S.S. 1967. Statistical Models in Engineering. New York, New York: John Wiley \& Sons. TIC: 247729.

Hardin, E.L. 1998. Near-Field/Altered-Zone Models Report. UCRL-ID-129179 DR. Livermore, California: Lawrence Livermore National Laboratory. ACC: MOL.19980504.0577.

Howard, C.L.; Finley, R.L.; Johnston, R.L.; Taylor, R.S.; George, J.T.; Lowry, W.E.; and Mason, N.G. 2001. Engineered Barrier System-Pilot Scale Test \#3, Heated Drip Shield Test 
Results. TDR-EBS-SE-000001 REV 00. Las Vegas, Nevada: Bechtel SAIC Company. ACC: MOL.20010529.0330.

Incropera, F.P. and DeWitt, D.P. 1996. Fundamentals of Heat and Mass Transfer. 4th Edition. New York, New York: John Wiley \& Sons. TIC: 243950.

Isaaks, E.H. and Srivastava, R.M. 1989. Applied Geostatistics. New York, New York: Oxford University Press. TIC: 200301.

Keenan, J.H.; Keyes, F.G.; Hill, P.G.; and Moore, J.G. 1969. Steam Tables, Thermodynamic Properties of Water Including Vapor, Liquid, and Solid Phases (English Units). New York, New York: John Wiley \& Sons. TIC: 246766.

Kitanidis, P.K. 1997. Introduction to Geostatistics: Applications to Hydrogeology. New York, New York: Cambridge University Press. TIC: 236758.

Kunii, D. and Smith, J.M. 1960. "Heat Transfer Characteristics of Porous Rocks." American Institute of Chemical Engineers Journal, 6, (1), 71-78. [New York, New York: American Institute of Chemical Engineers]. TIC: 249321.

Leem, J. 2001. "Delivery of Thermal-Hydrology Sensitivity Calculations for Pre-Closure Ventilation Efficiency and Ventilation Duration for the SSPA Volume 1 Report." Memorandum from J. Leem (BSC) to J. Blink, July 17, 2001, PROJ.07/01.029, with enclosure. ACC: MOL.20010815.0301.

McNeish, J. 2001. "Preliminary REV 00A Version of FY01 Supplemental Science and Performance Analyses, Volume 2: Performance Analyses, TDR-MGR-PA-000001." Memorandum from J. McNeish (BSC) to RPC = 4, May 29, 2001, PROJ.05/01.063, with enclosure. ACC: MOL.20010529.0377.

Mongano, G.S.; Singleton, W.L.; Moyer, T.C.; Beason, S.C.; Eatman, G.L.W.; Albin, A.L.; and Lung, R.C. 1999. Geology of the ECRB Cross Drift - Exploratory Studies Facility, Yucca Mountain Project, Yucca Mountain, Nevada. [Deliverable SPG42GM3]. Denver, Colorado: U.S. Geological Survey. ACC: MOL.20000324.0614.

Phillips, O.M. 1991. Flow and Reactions in Permeable Rocks. New York, New York: Cambridge University Press. On Order Library Tracking Number-236237.

Sass, J.H.; Lachenbruch, A.H.; Dudley, W.W., Jr.; Priest, S.S.; and Munroe, R.J. 1988. Temperature, Thermal Conductivity, and Heat Flow Near Yucca Mountain, Nevada: Some Tectonic and Hydrologic Implications. Open-File Report 87-649. [Denver, Colorado]: U.S. Geological Survey. TIC: 203195.

Wilder, D.G., ed. 1996. Volume II: Near-Field and Altered-Zone Environment Report. UCRLLR-124998. [Livermore, California]: Lawrence Livermore National Laboratory. ACC: MOL.19961212.0121; MOL.19961212.0122. 


\subsection{Procedures Cited}

AP-3.12Q, Rev. 0, ICN 4. Calculations. Washington, D.C.: U.S. Department of Energy, Office of Civilian Radioactive Waste Management. ACC: MOL.20010404.0008.

AP-SI.1Q, Rev. 2, ICN 4. Software Management. Washington, D.C.: U.S. Department of Energy, Office of Civilian Radioactive Waste Management. ACC: MOL.20000223.0508.

\subsection{Source Data}

LB990701233129.001. 3-D UZ Model Grids for Calculation of Flow Fields for PA for AMR U0000, "Development of Numerical Grids for UZ Flow and Transport Modeling". Submittal date: $09 / 24 / 1999$.

LB990801233129.001. TSPA Grid Flow Simulations for AMR U0050, "UZ Flow Models and Submodels" (Flow Field \#1). Submittal date: 11/29/1999.

LB990801233129.003. TSPA Grid Flow Simulations for AMR U0050, "UZ Flow Models and Submodels" (Flow Field \#3). Submittal date: 11/29/1999.

LB990801233129.005. TSPA Grid Flow Simulations for AMR U0050, "UZ Flow Models and Submodels" (Flow Field \#5). Submittal date: 11/29/1999.

LB990801233129.007. TSPA Grid Flow Simulations for AMR U0050, "UZ Flow Models and Submodels" (Flow Field \#7). Submittal date: 11/29/1999

LB990801233129.009. TSPA Grid Flow Simulations for AMR U0050, "UZ Flow Models and Submodels" (Flow Field \#9). Submittal date: 11/29/1999

LB990801233129.011. TSPA Grid Flow Simulations for AMR U0050, "UZ Flow Models and Submodels" (Flow Field \#11). Submittal date: 11/29/1999.

LB990801233129.013. TSPA Grid Flow Simulations for AMR U0050, "UZ Flow Models and Submodels" (Flow Field \#13). Submittal date: 11/29/1999.

LB990801233129.015. TSPA Grid Flow Simulations for AMR U0050, "UZ Flow Models and Submodels" (Flow Field \#15). Submittal date: 11/29/1999.

LB990801233129.017. TSPA Grid Flow Simulations for AMR U0050, "UZ Flow Models and Submodels" (Flow Field \#17). Submittal date: 11/29/1999.

LB990861233129.001. Drift Scale Calibrated 1-D Property Set, FY99. Submittal date: 08/06/1999.

LB990861233129.002. Drift Scale Calibrated 1-D Property Set, FY99. Submittal date: 08/06/1999.

LB990861233129.003. Drift Scale Calibrated 1-D Property Set, FY99. Submittal date: 08/06/1999. 
LB991091233129.006. Thermal Properties and Tortuosity Factor for the UZ Model Layers for AMR U0090, "Analysis of Hydrologic Properties Data". Submittal date: 10/15/1999.

LB991201233129.001. The Mountain-Scale Thermal-Hydrologic Model Simulations for AMR U0105, "Mountain-Scale Coupled Processes (TH) Models". Submittal date: 03/11/2000.

MO9901RIB00044.000. Reference Information Base Data Item - Hydrologic Characteristics: Unsaturated Zone Flow Characteristics. Submittal date: 01/06/1999.

SN9908T0872799.004. Tabulated In-Drift Geometric and Thermal Properties Used in DriftScale Models for TSPA-SR (Total System Performance Assessment-Site Recommendation). Submittal date: 08/30/1999.

\subsection{Software Sources}

Lawrence Livermore National Laboratory 2001. chim_surf_wt_TP2. 1.0. (TBV)

Lawrence Livermore National Laboratory 2001. columnInfiltration. 1.2. (TBV)

Lawrence Livermore National Laboratory 2001. LDTH-01-li-i. 1.0. Sun Ultra 10. (TBV)

Lawrence Livermore National Laboratory 2001. LDTH-01-li-v. 1.0. Sun Ultra 10. (TBV)

Lawrence Livermore National Laboratory 2001. LDTH-01-li. 1.0. Sun Ultra 10. (TBV)

Lawrence Livermore National Laboratory 2001. LDTH-01-mi-i. 1.0. Sun Ultra 10. (TBV)

Lawrence Livermore National Laboratory 2001. LDTH-01-mi-v. 1.0. Sun Ultra 10. (TBV)

Lawrence Livermore National Laboratory 2001. LDTH-01-mi. 1.0. Sun Ultra 10. (TBV)

Lawrence Livermore National Laboratory 2001. LDTH-01-ui-i. 1.0. Sun Ultra 10. (TBV)

Lawrence Livermore National Laboratory 2001. LDTH-01-ui-v. 1.0. Sun Ultra 10. (TBV)

Lawrence Livermore National Laboratory 2001. LDTH-01-ui. 1.0. Sun Ultra 10. (TBV)

Lawrence Livermore National Laboratory 2001. LDTH-03-li-v. 1.0. Sun Ultra 10. (TBV)

Lawrence Livermore National Laboratory 2001. LDTH-03-li. 1.0. Sun Ultra 10. (TBV)

Lawrence Livermore National Laboratory 2001. LDTH-03-mi-v. 1.0. Sun Ultra 10. (TBV)

Lawrence Livermore National Laboratory 2001. LDTH-03-mi. 1.0. Sun Ultra 10. (TBV)

Lawrence Livermore National Laboratory 2001. LDTH-03-ui-v. 1.0. Sun Ultra 10. (TBV)

Lawrence Livermore National Laboratory 2001. LDTH-03-ui. 1.0. Sun Ultra 10. (TBV) 
Lawrence Livermore National Laboratory 2001. MSTHAC. 6.4.3. Sun Ultra 10. (TBV)

Lawrence Livermore National Laboratory 2001. rock_LDTH. 1.0. Sun Ultra 10. (TBV)

Lawrence Livermore National Laboratory 2001. rock_SDT. 1.0. Sun Ultra 10. (TBV)

Lawrence Livermore National Laboratory 2001. SDT-03. 1.0. Sun Ultra 10. (TBV)

Lawrence Livermore National Laboratory 2001. SDT-03v. 1.0. Sun Ultra 10. (TBV)

Lawrence Livermore National Laboratory 2001. SMT_bot_bc3. 1.0. Sun Ultra 10. (TBV)

Lawrence Livermore National Laboratory 2001. SMT_surf_bc3. 1.0. Sun Ultra 10. (TBV)

Lawrence Livermore National Laboratory 2001. StudyDomain. 1.0. Sun Ultra 10. (TBV)

Lawrence Livermore National Laboratory 2000. Software reference: infiltab.m V1.0. V1.0.

Sun Ultra10. ANL-EBS-MD-000049.

Lawrence Livermore National Laboratory 2000. Software routine: SDT-01 V1.0. V1.0. Sun Ultra10. ANL-EBS-MD-000049.

Lawrence Livermore National Laboratory 2000. Software routine: SDT-01i V1.0. V1.0. Sun Ultra10. ANL-EBS-MD-000049.

Lawrence Livermore National Laboratory 2000. Software routine: SDT-01v V1.0. V1.0. Sun Ultra10. ANL-EBS-MD-000049.

Lawrence Livermore National Laboratory 2000. Software routine: addlay V1.0. V1.0. Sun Ultra10. ANL-EBS-MD-000049.

Lawrence Livermore National Laboratory 2000. Software routine: addLayers V1.0. V1.0. Sun Ultra10. ANL-EBS-MD-000049.

Lawrence Livermore National Laboratory 2000. Software routine: bound.m V1.0. V1.0. Sun Ultra10. ANL-EBS-MD-000049.

Lawrence Livermore National Laboratory 2000. Software Routine: CONVERTCOORDS V1.1. V1.1. 10209-1.1-00.

Lawrence Livermore National Laboratory 2000. Software routine: heatgenAge V1.2. V1.2. Sun Ultra10. ANL-EBS-MD-000049.

Lawrence Livermore National Laboratory 2000. Software routine: MakeColumns V1.0. V1.0. Sun Workshop SC4.2 cc compiler. ANL-EBS-MD-000049. column_template and column.data.

Lawrence Livermore National Laboratory 2000. Software routine: RADPRO V3.22. V3.22. Sun Ultra10. 10204-3.22-00. 
Lawrence Livermore National Laboratory 2000. Software routine: readsUnits V1.0. V1.0. Sun Ultra10. ANL-EBS-MD-000049.

Lawrence Livermore National Laboratory 2000. Software routine: XTOOL V10.1. V10.1. Sun Ultra10. 10208-10.1-00.

Lawrence Livermore National Laboratory 2000. Software Routine: YMESH V1.53. V1.53.

10172-1.53-00.

LLNL (Lawrence Livermore National Laboratory) 1999. Software Code: NUFT V3.0s.. V3.0s. 10088-3.0s-00.

LLNL (Lawrence Livermore National Laboratory) 2000. Software routine: rme6 V1.1. V1.1. Sum Ultra10. ANL-EBS-MD-000032. 


\section{FIGURES}

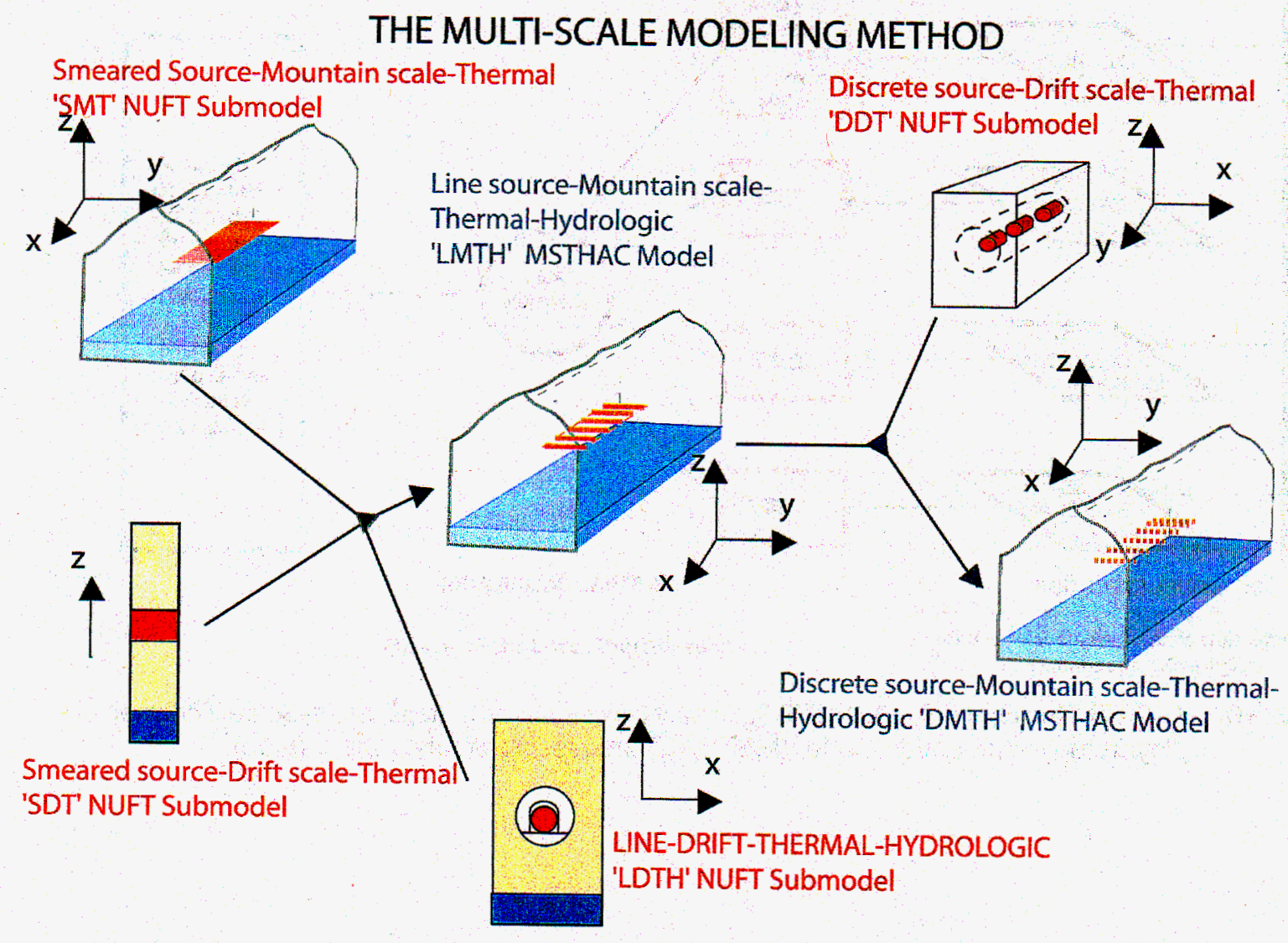

Figure 1 The Multi-Scale Thermal-Hydrologic (MSTH) modeling approach. Shown are the constructive relation between the four NUFT submodels (in red text) and the two MSTHAC submodels (in blue text). 


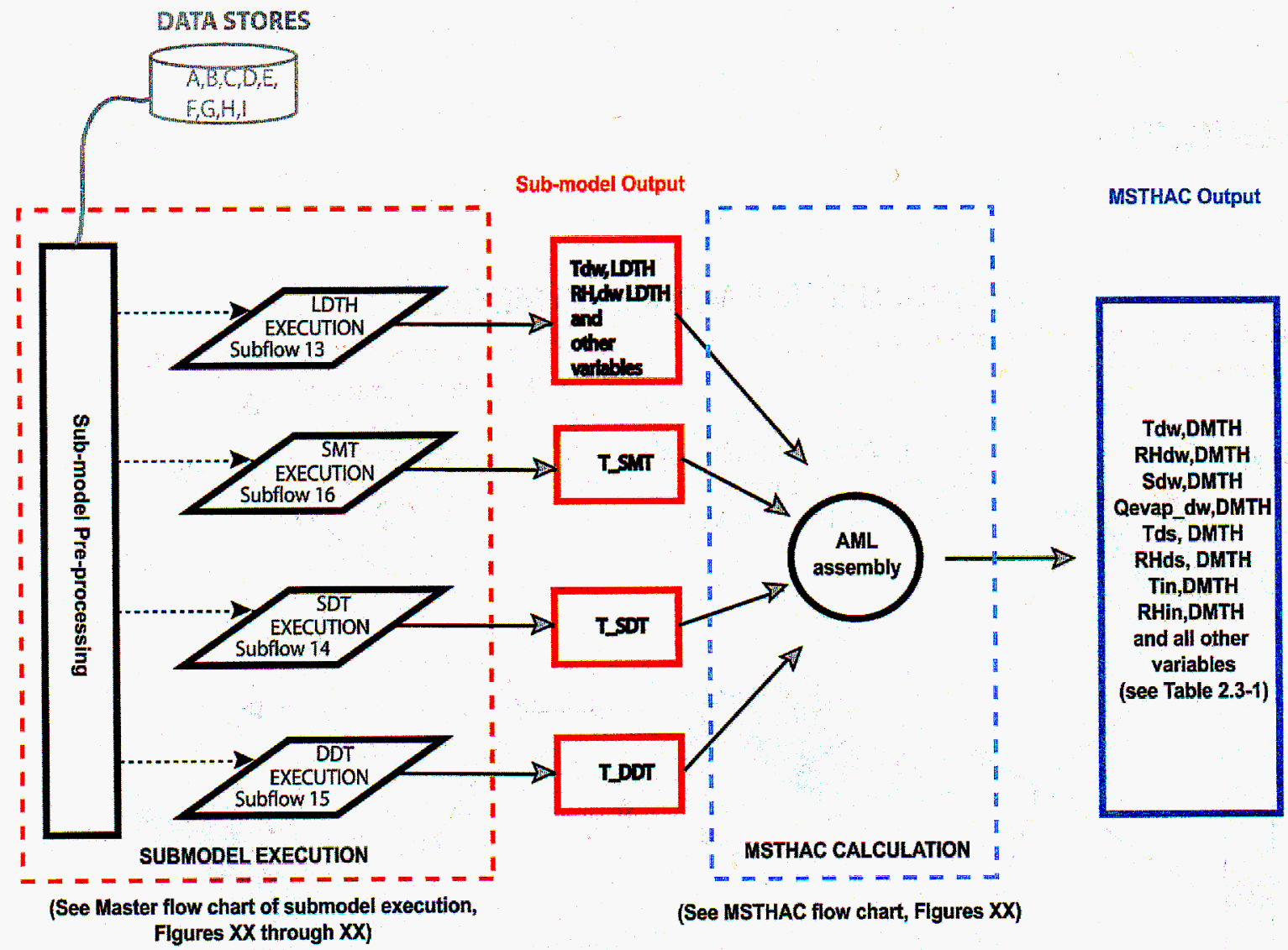

Figure 2 MSTH Model Flowchart: Step1, NUFT Submodel execution (red); Step2, Multi-Scale Thermal Hydrology Abstraction Code (MSTHAC) processing of final output (blue). 


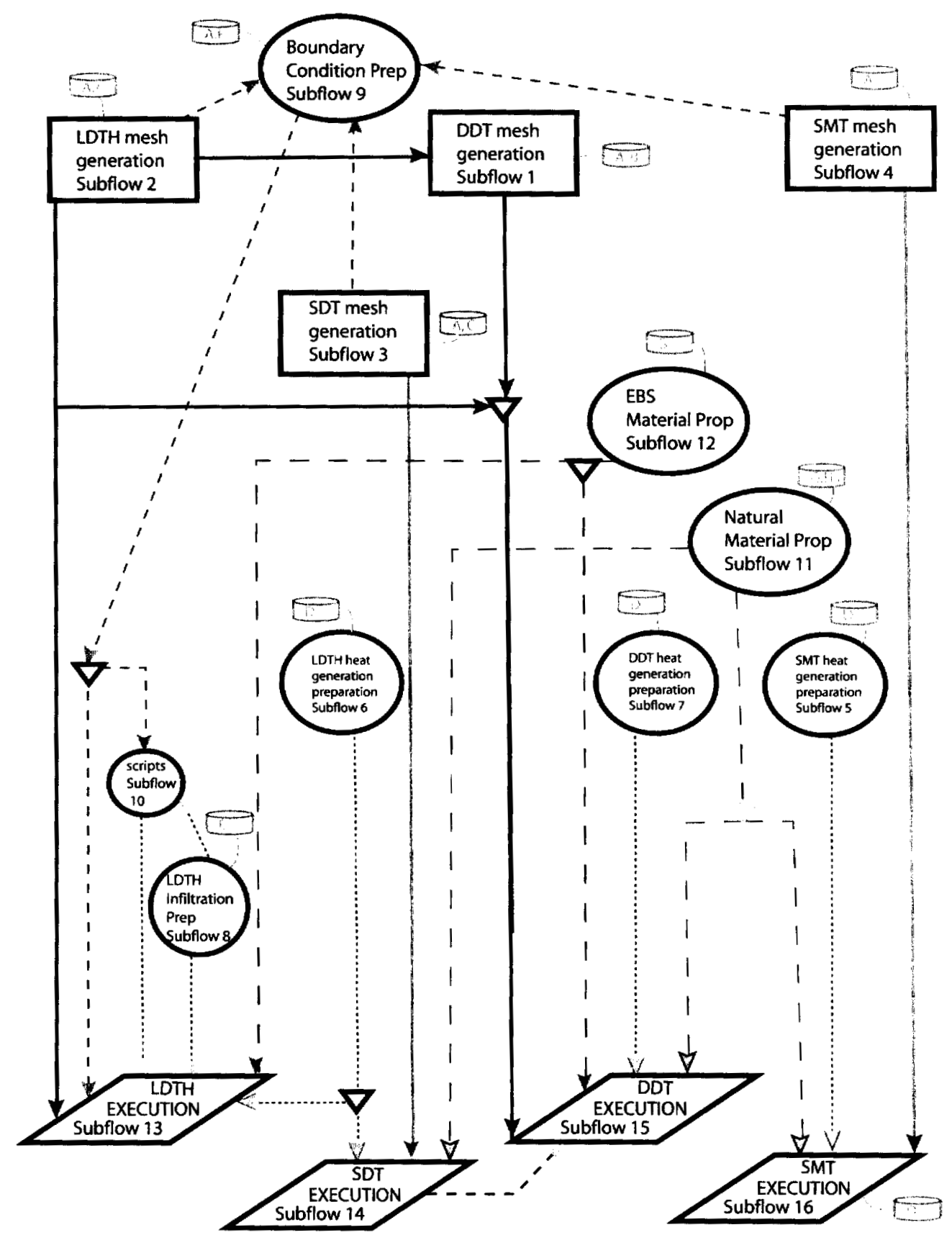

Figure 3 Master Flowchart of the NUFT submodel execution process. Each box on this chart refers to a 'Subflow' which is the Sub-flowcharts below. Note the Data Stores providing input to the flowchart (stores A, B, C , D, E, F, G, H, I Table 2. 


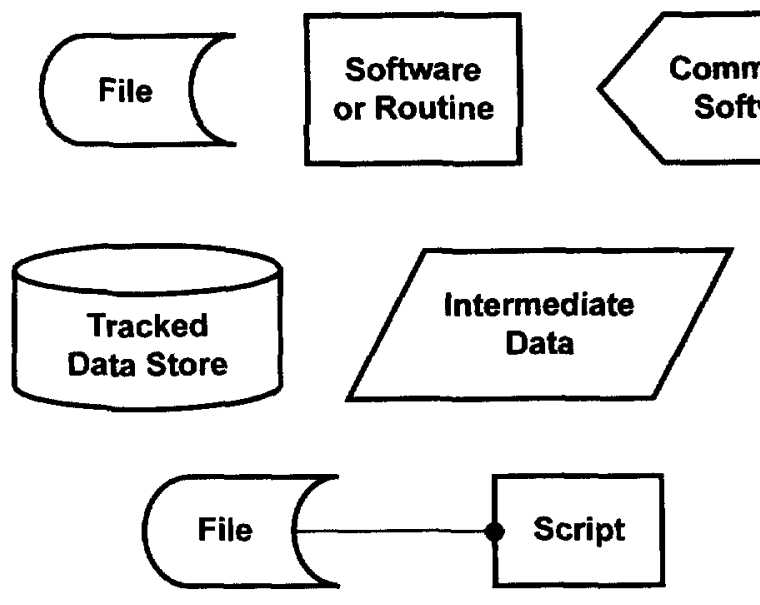

Indicates that File is executed as a Script

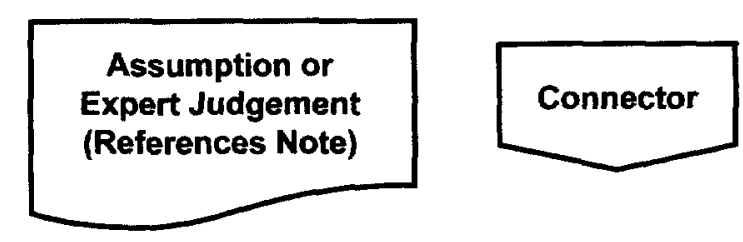

Manual Operation (References Table)

Figure 4 Legend to Sub-flowcharts 1 through 16, Figure 5 through Figure 20, respectively 


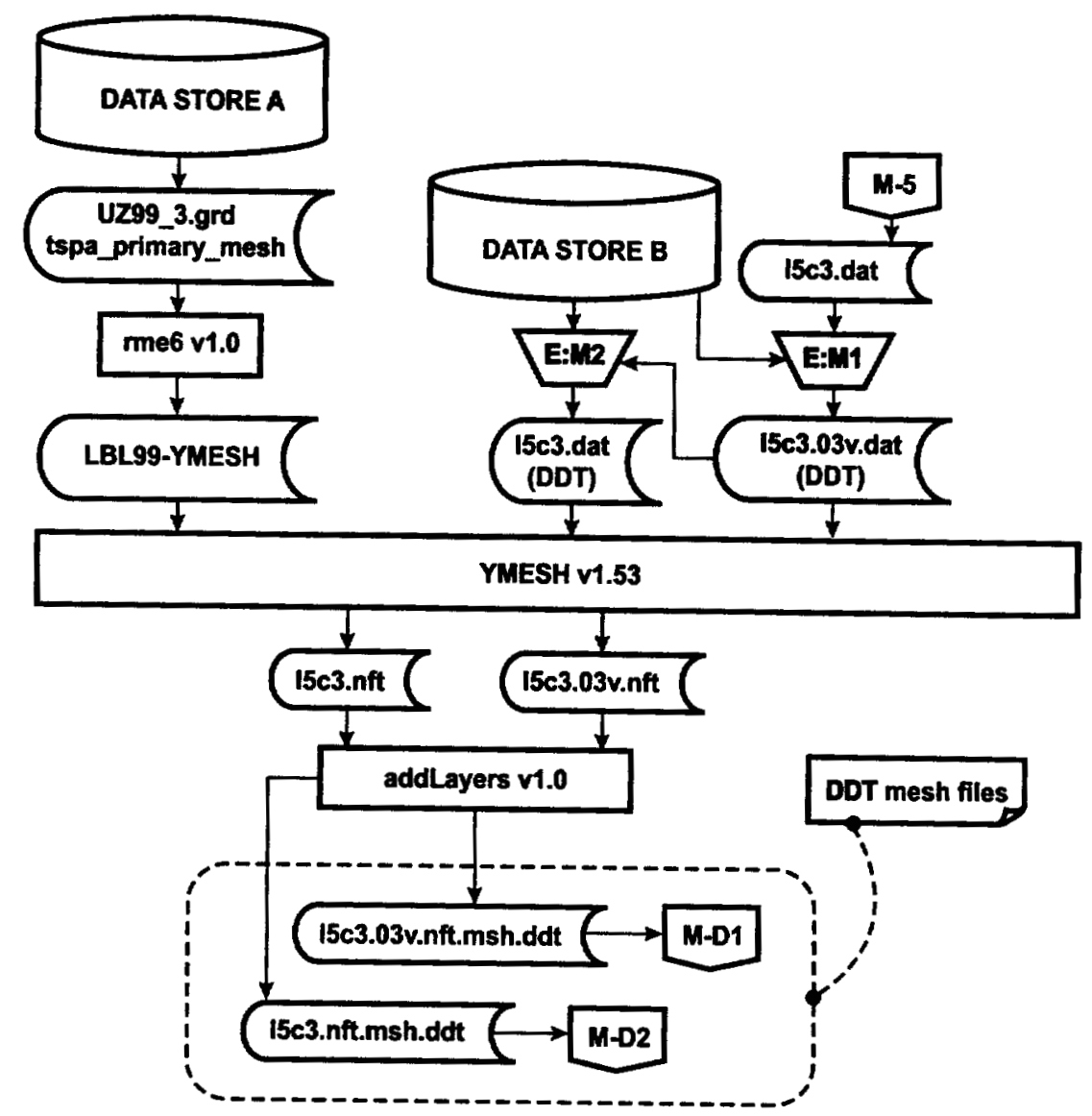

M-5 from Sub-flowchart, M-D1 to Sub-flowchart 15, M-D2 to Sub-flowchart 15

Figure 5 Sub-flowchart 1, DDT mesh generation. 


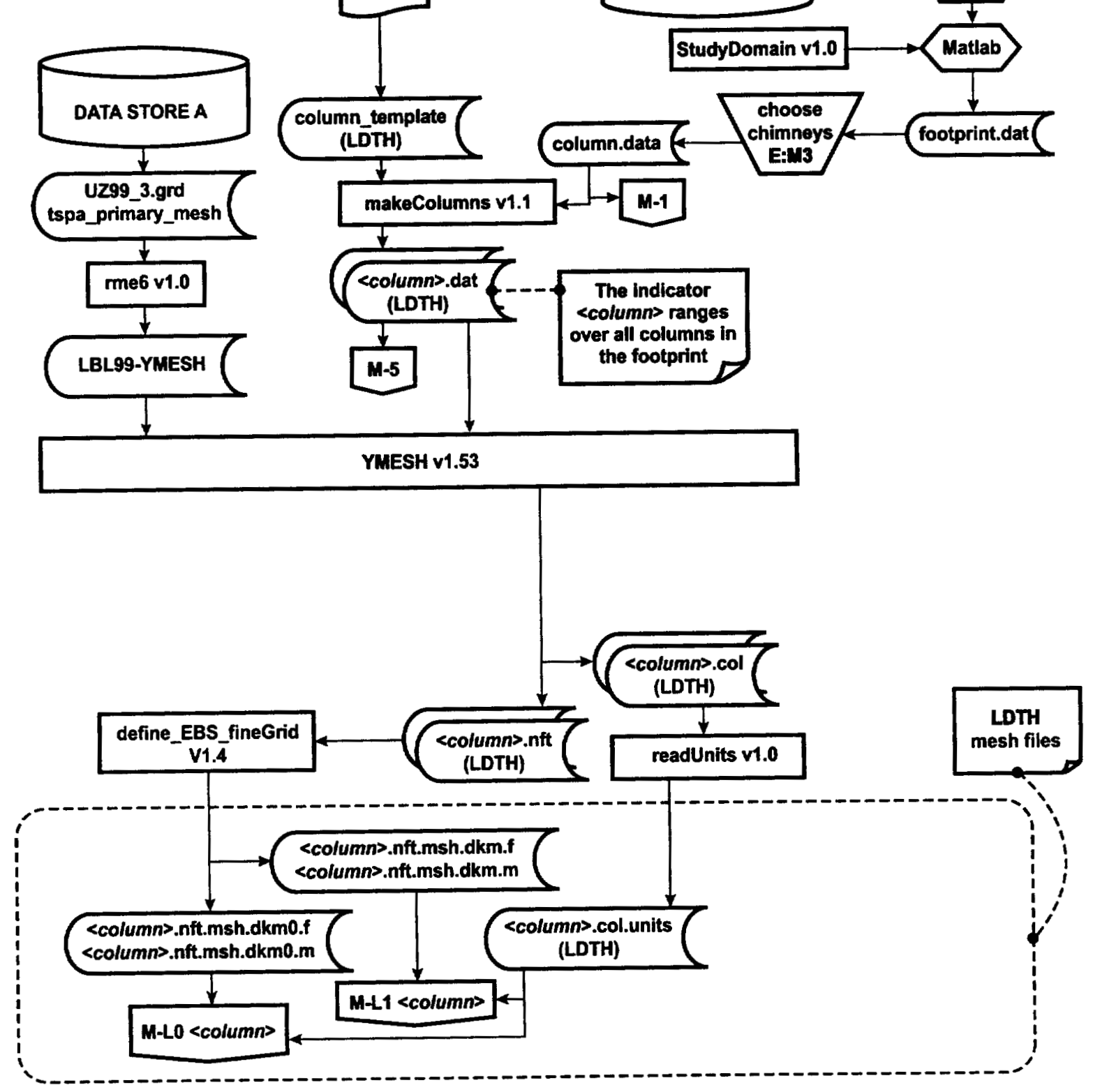

$M-1$ to Sub-flowchart 9, M-5 to Sub-flowchart $1, M-L 0$ and $M-L 1$ to Sub-flowchart 13

Figure 6 Sub-flowchart 2, LDTH mesh generation. 


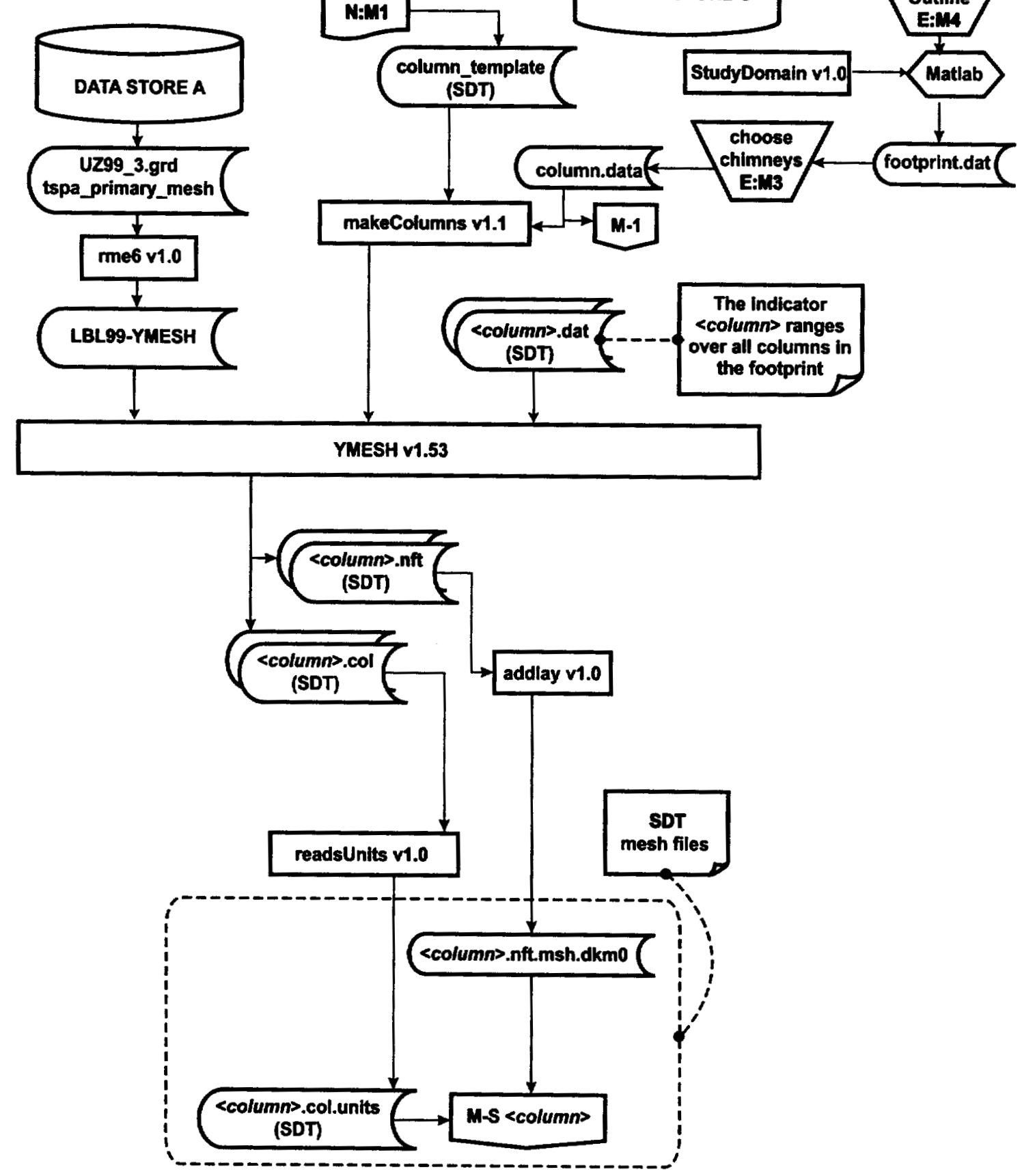

$M-1$ to Sub-flowchart 9, M-S to Sub-flowcharts 9 and 15

Figure 7 Sub-flowchart 3, SDT mesh generation. 


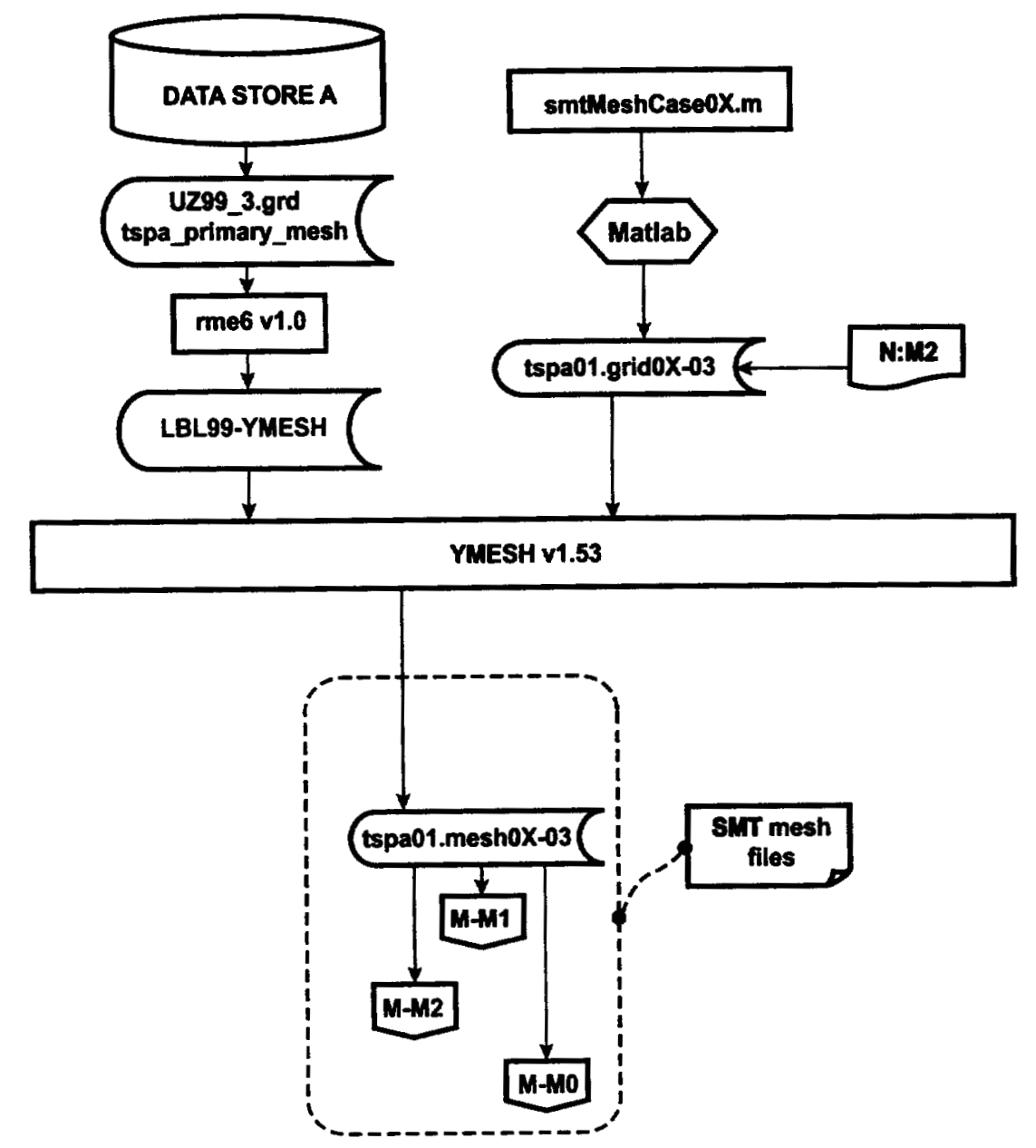

$M-M O$ to Sub-flowchart 9, M-M1 and M-M2 to Sub-flowchart 16

Figure 8 Sub-flowchart 4, SMT mesh generation. 


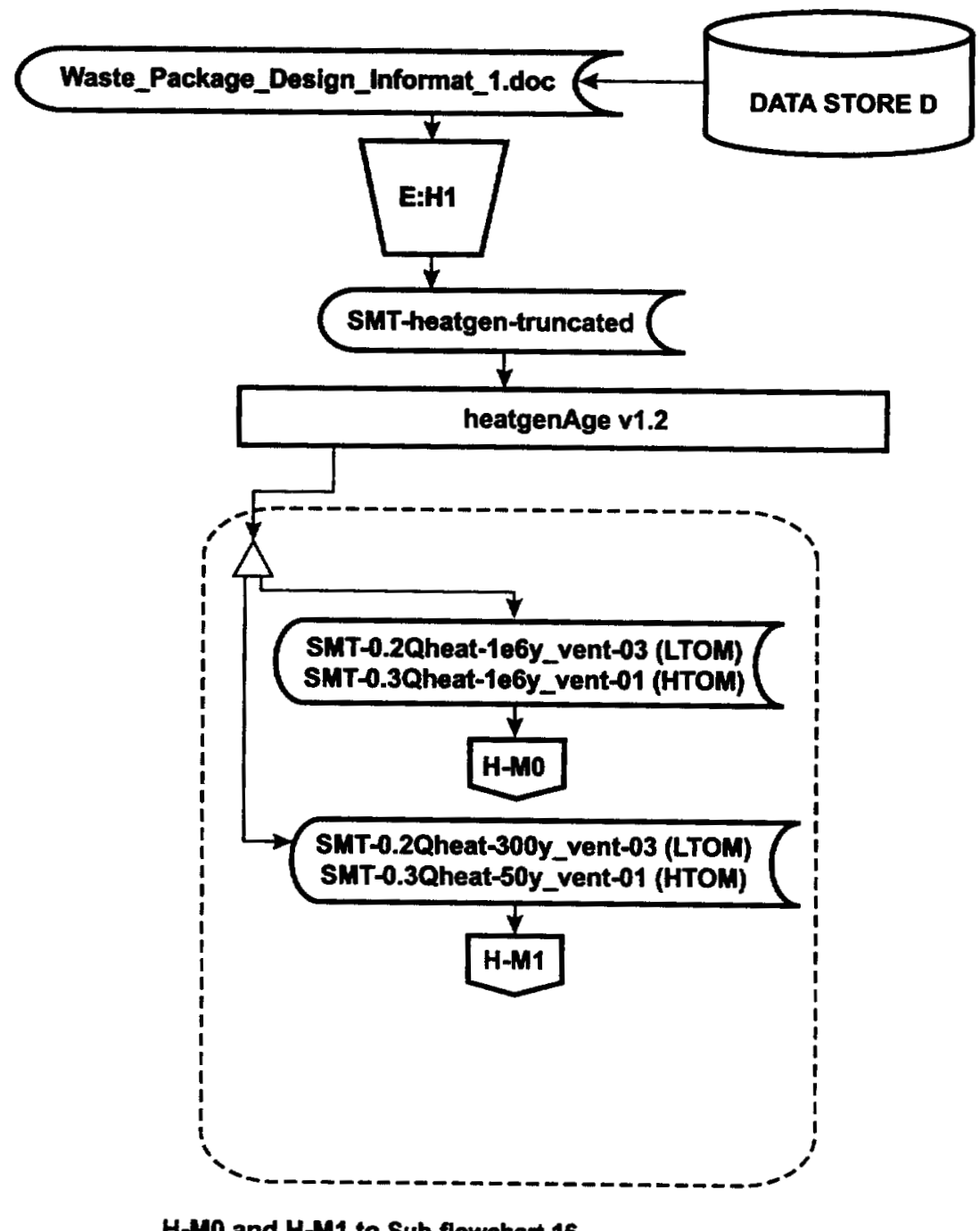

Figure 9 Sub-flowchart 5, SMT heat generation preparation. 


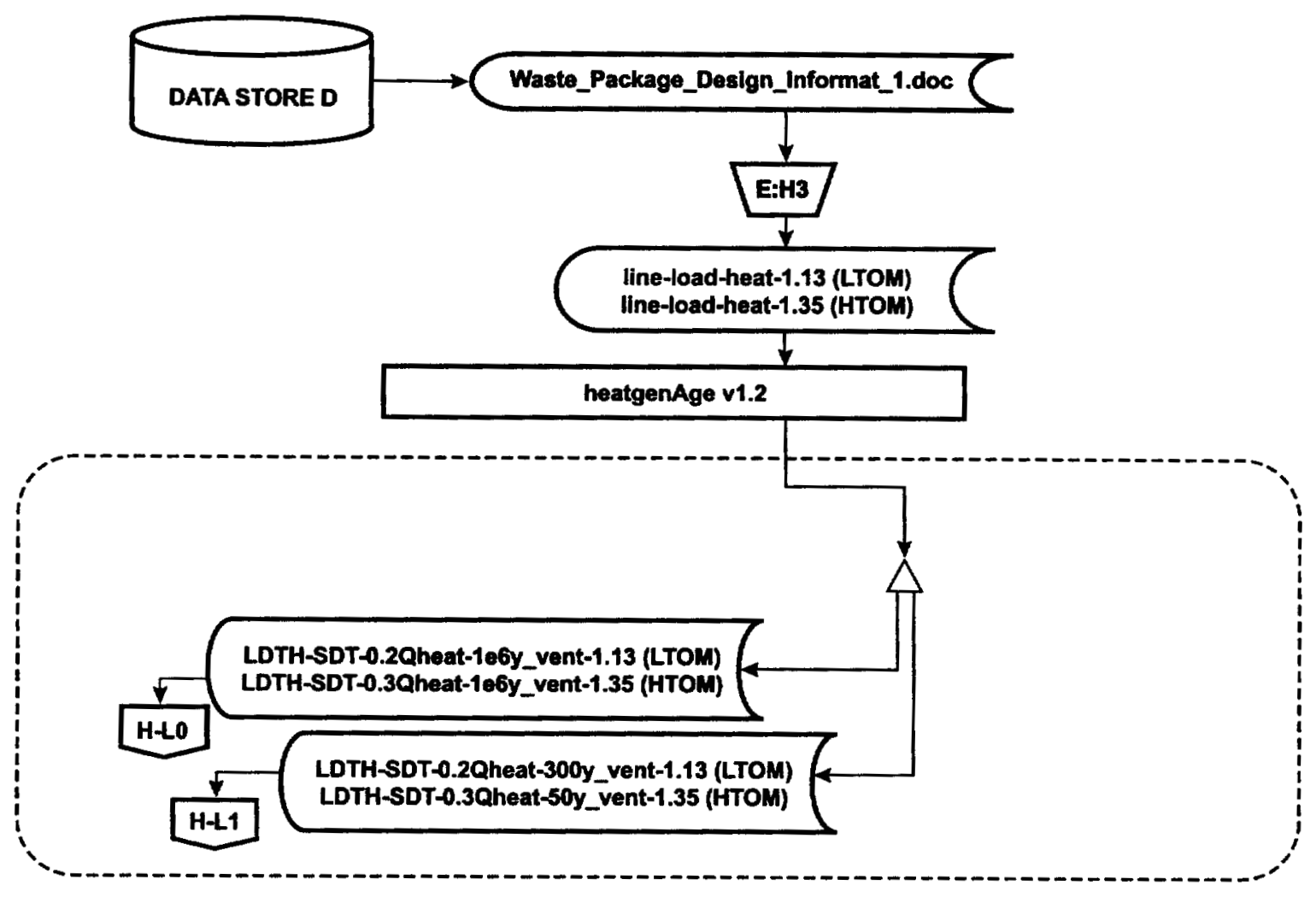

H-LO and H-L1 to Sub-flowcharts 13,14

Figure 10 Sub-flowchart 6, LDTH heat generation preparation. 


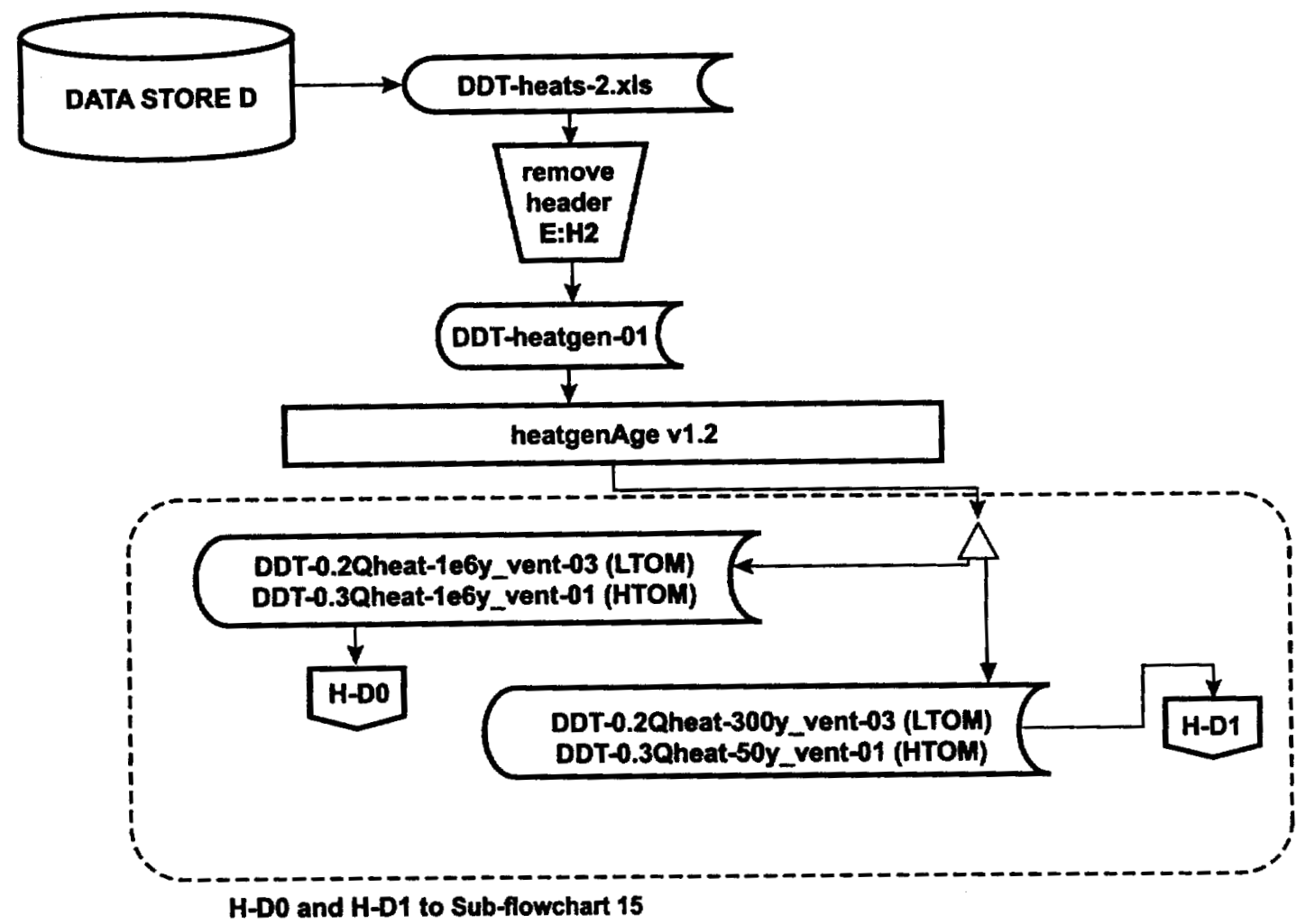

Figure 11 Sub-flowchart 7, DDT heat generation preparation. 


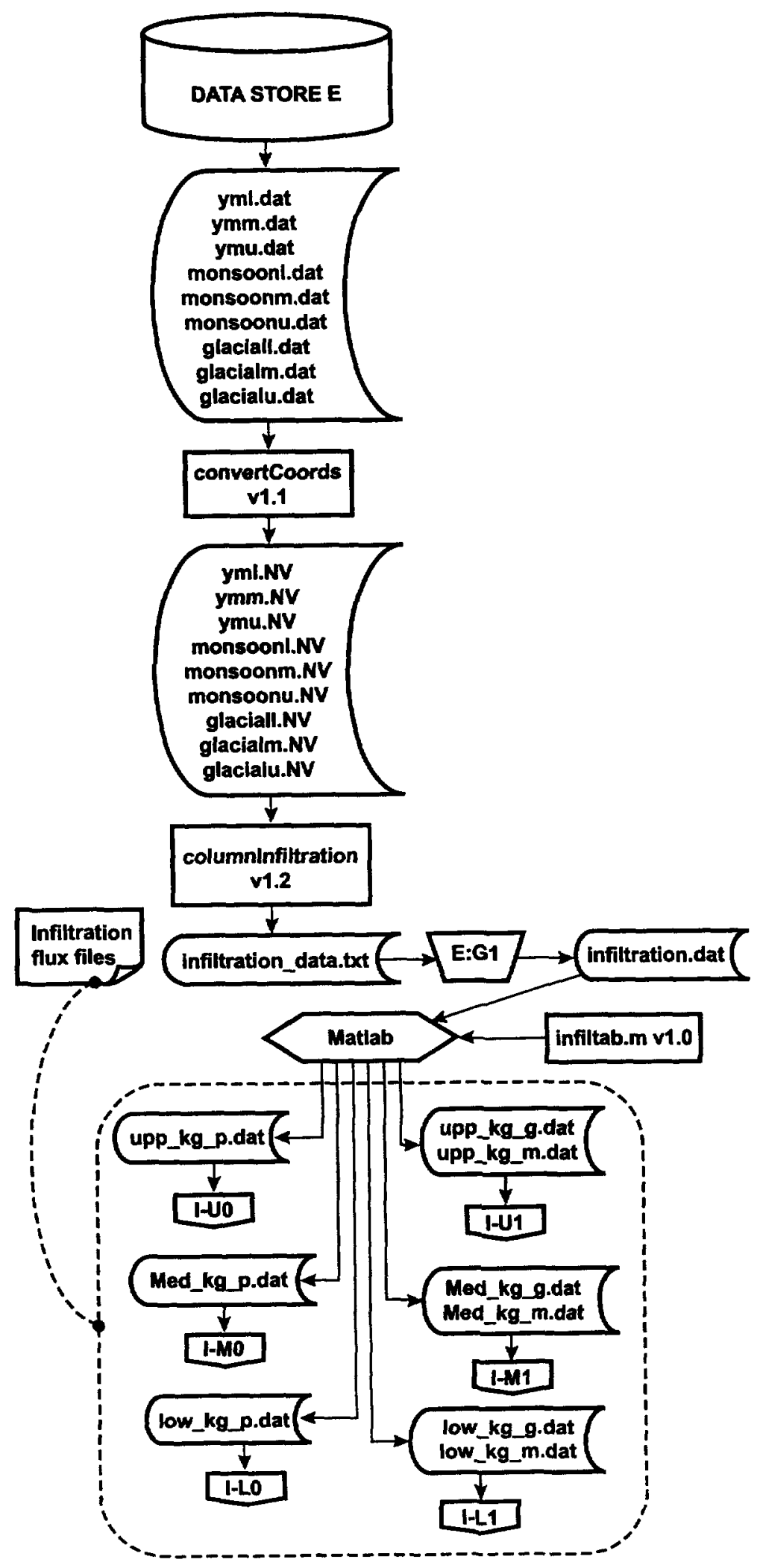

I-UO, I-U1, I-MO, I-M1, I-LO, I-L1 all to Sub-flowcharts 10 and 13

Figure 12 Sub-flowchart 8, LDTH infiltration preparation. 


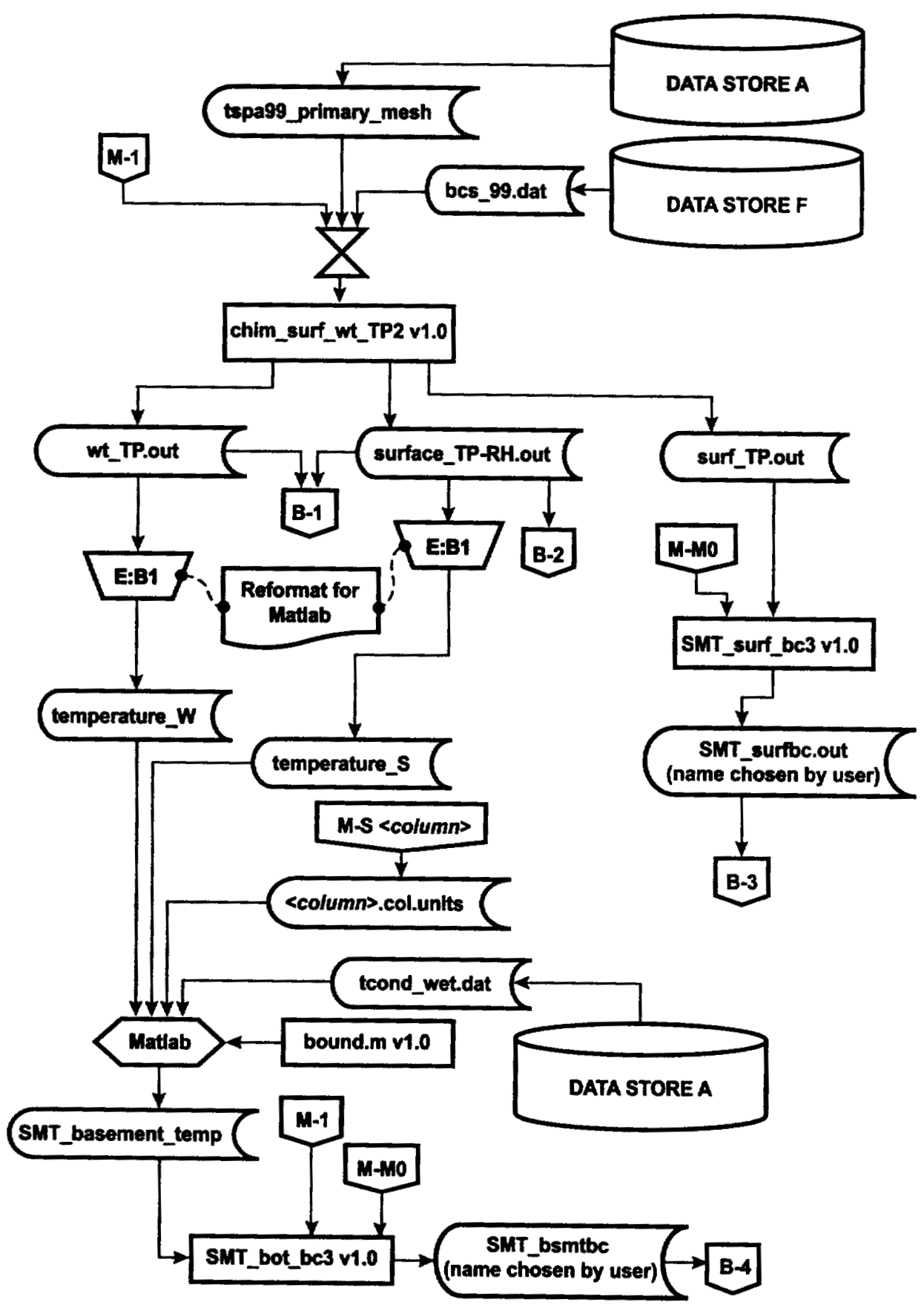

M-1 from Sub-flowchart 2 and 3; M-MO from Sub-flowchart 9; M-S from Sub-flowcharts 9 and 14

B-1 to Sub-flowcharts 10, 13, and 14; B-2 to Sub-flowehart 10; B-3 and B-4 to Sub-flowchart 16

Figure 13 Sub-flowchart 9, boundary condition preparation. 


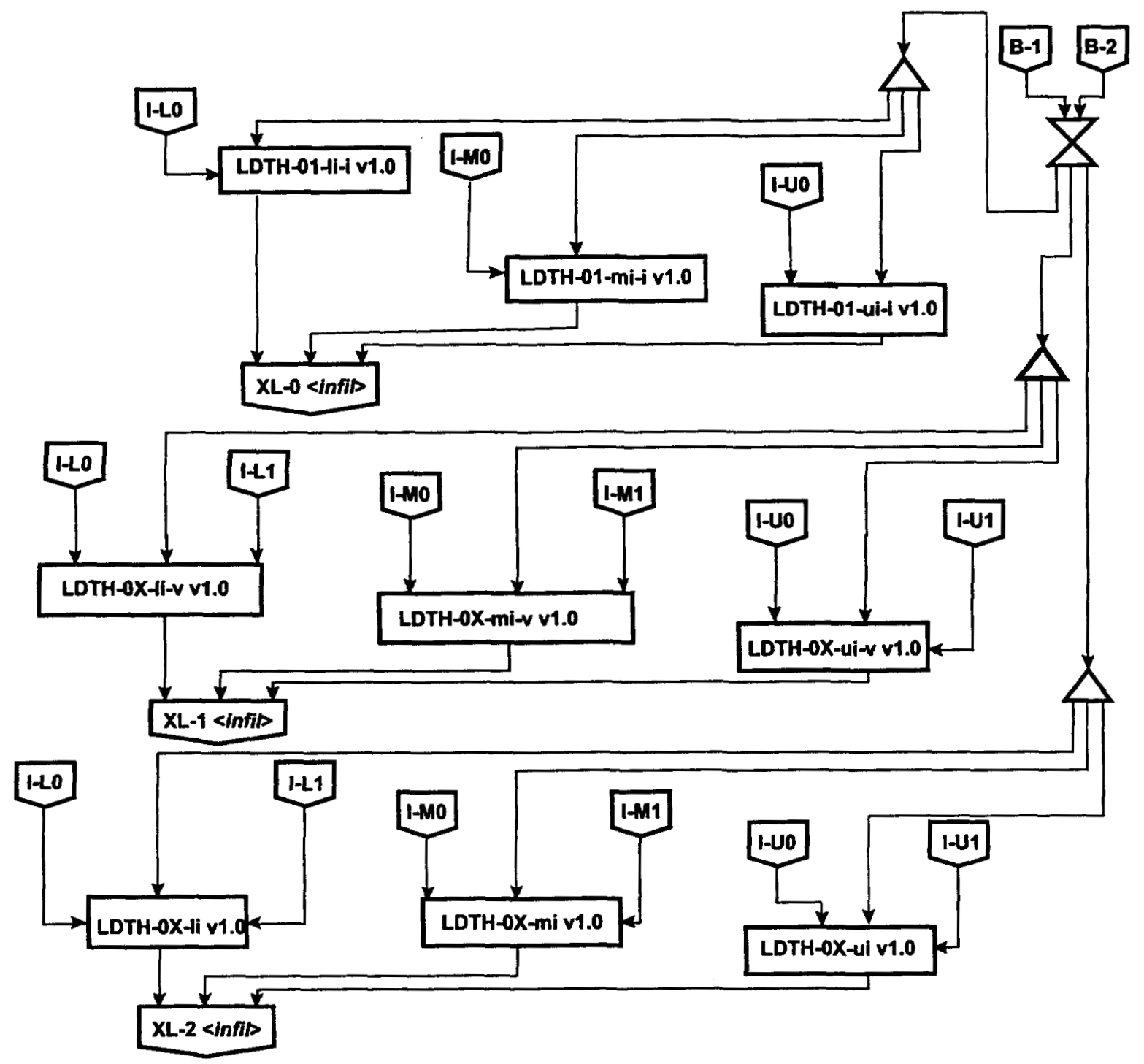

I-LO, I-L1, I-HO, I-M1, I-UO, and I-U1 from Sub-flowcharts 10 and 13; B-1 and B-2 from Sub-flowchart 9

$X L-0<$ infil>, $X L-1<i n f i l$, and $X L-2<$ infil> to Sub-flowchart 13

Figure 14 Sub-flowchart 10, LDTH scripts. 


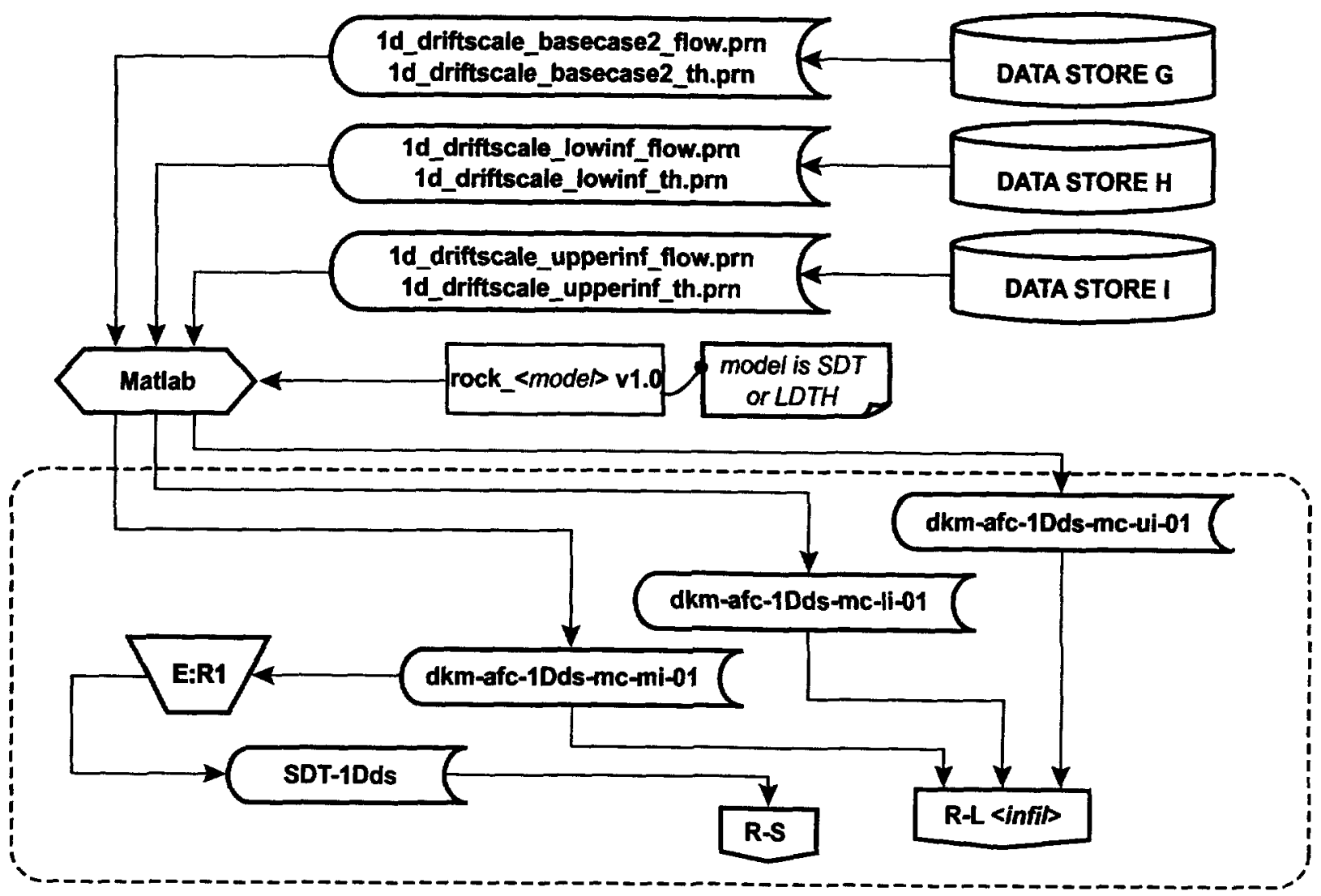

R-L<infil> to Sub-flowchart 13; R-S to Sub-flowcharts 14,15 , and 16

Figure 15 Sub-flowchart 11, natural material properties. 


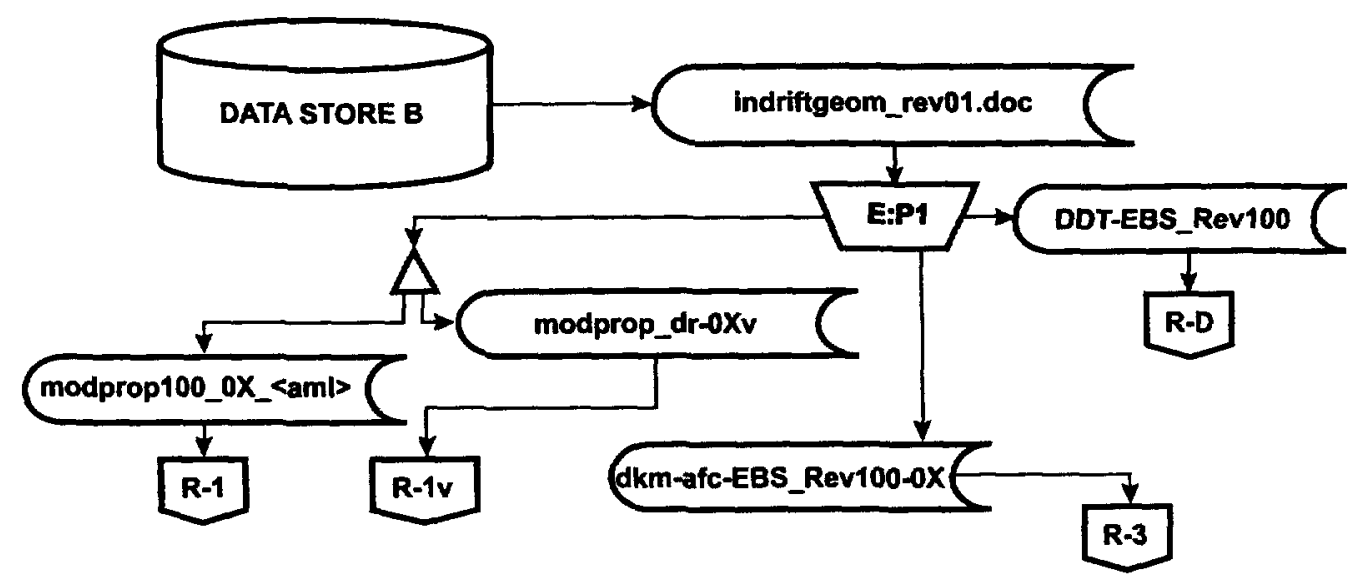

R-1 to Sub-flowcharts 13 and 15; R-1v and R-3 to Sub-flowchart 13; R-D to Sub-flowchart 15

Figure 16 Sub-flowchart 12, EBS material properties. 


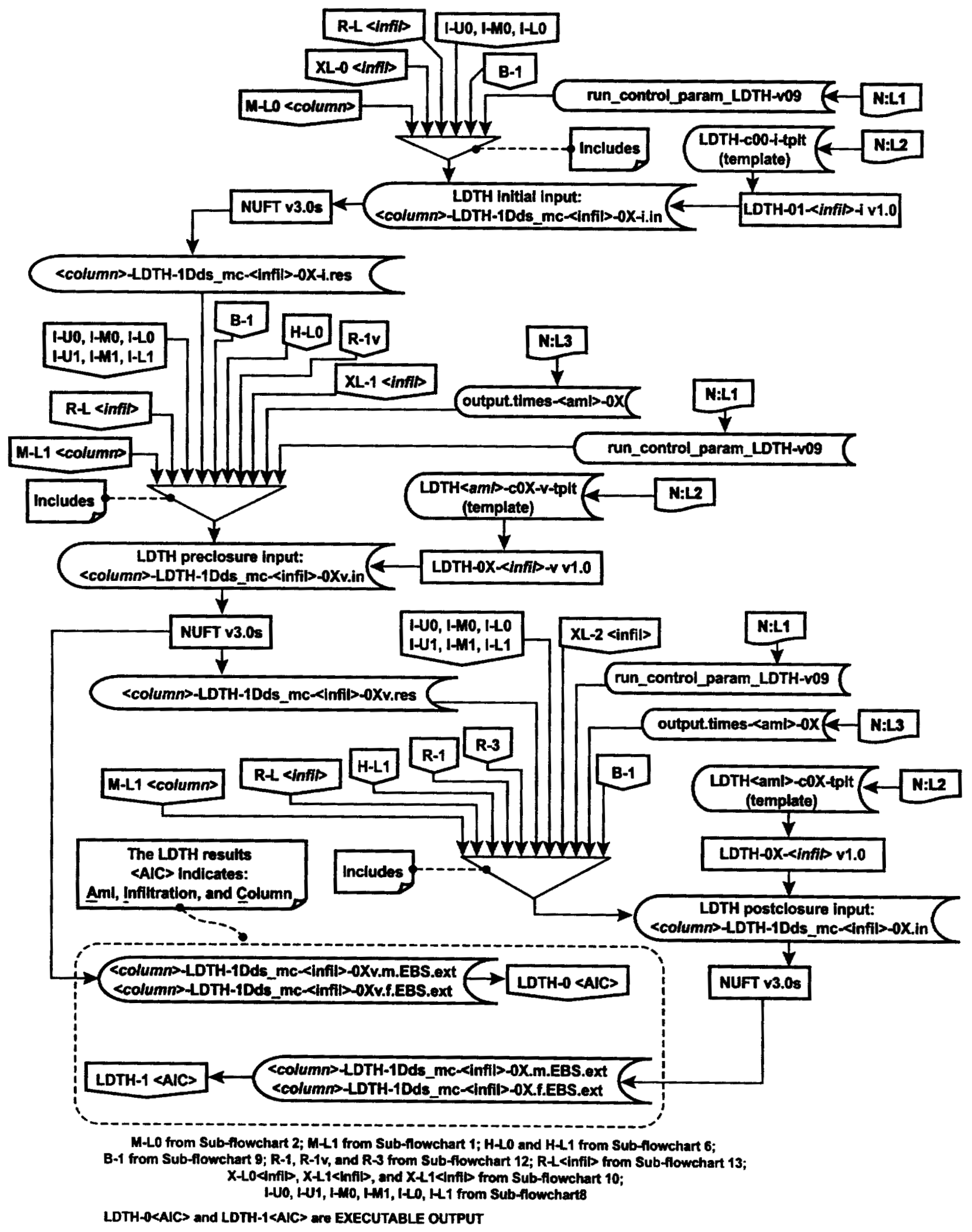

Figure 17 Sub-flowchart 13, LDTH submodel execution. 


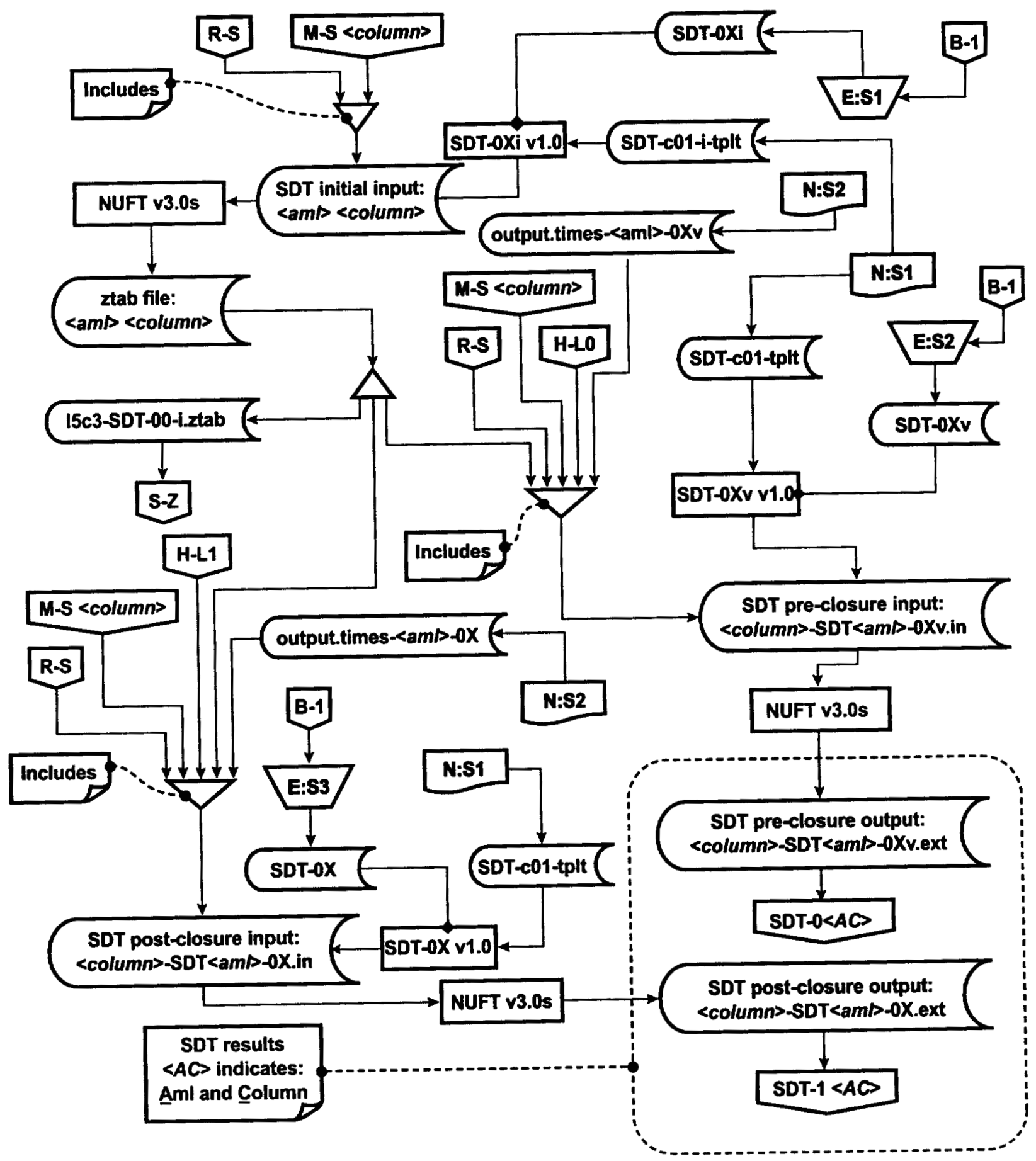

$M-S$ from Sub-flowchart 3; H-LO and H-L1 from Sub-flowchart 6;

B-1 from Sub-flowchart 9; R-S from Sub-flowchart 14, 15, and 16;

S-Z to Sub-flowchart 15

SDT-O<AC> and SDT-1<AC> are EXECUTABLE OUTPUT

Figure 18 Sub-flowchart 14, SDT submodel execution. 


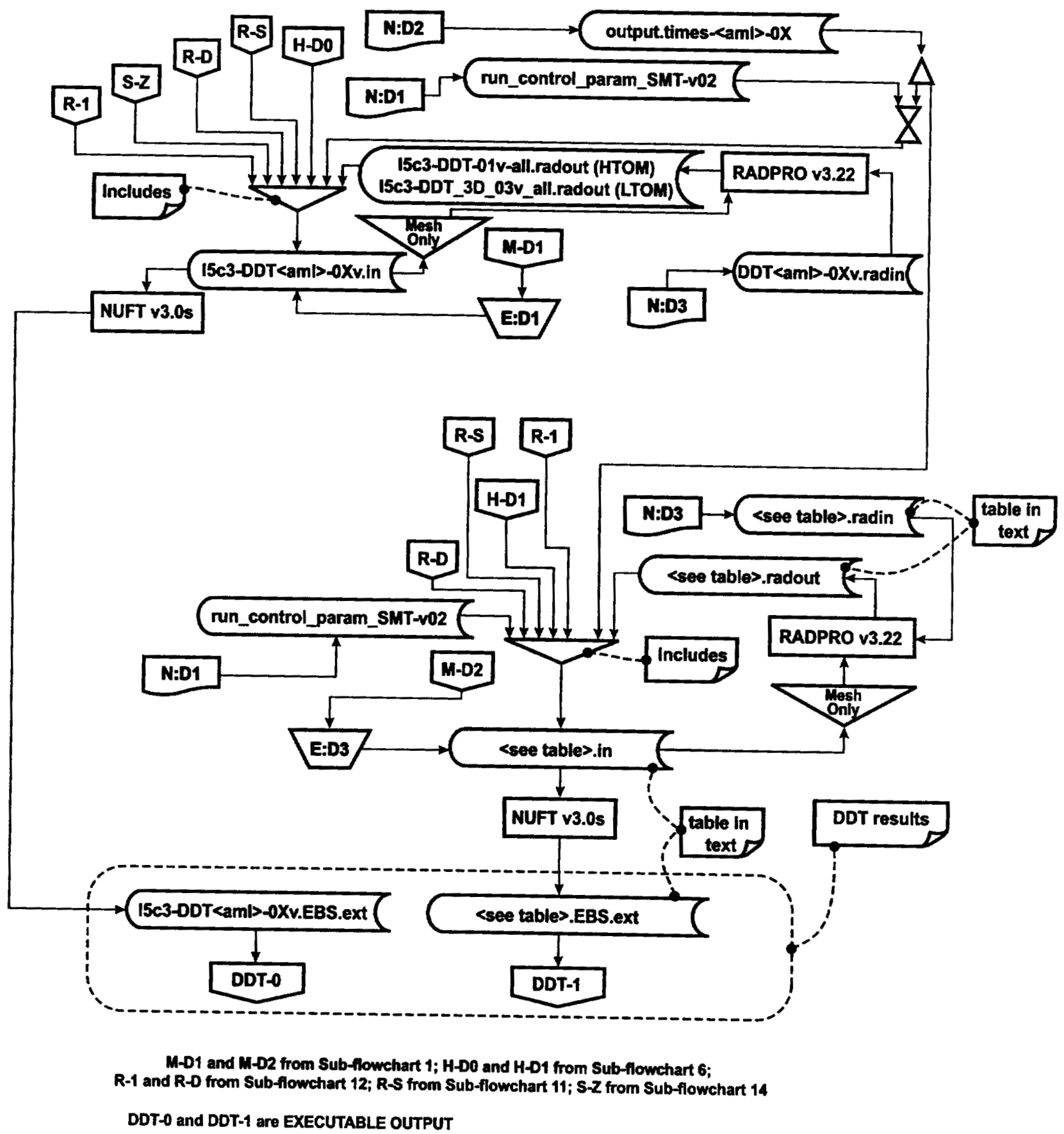

Figure 19 Sub-flowchart 15, DDT submodel execution. 


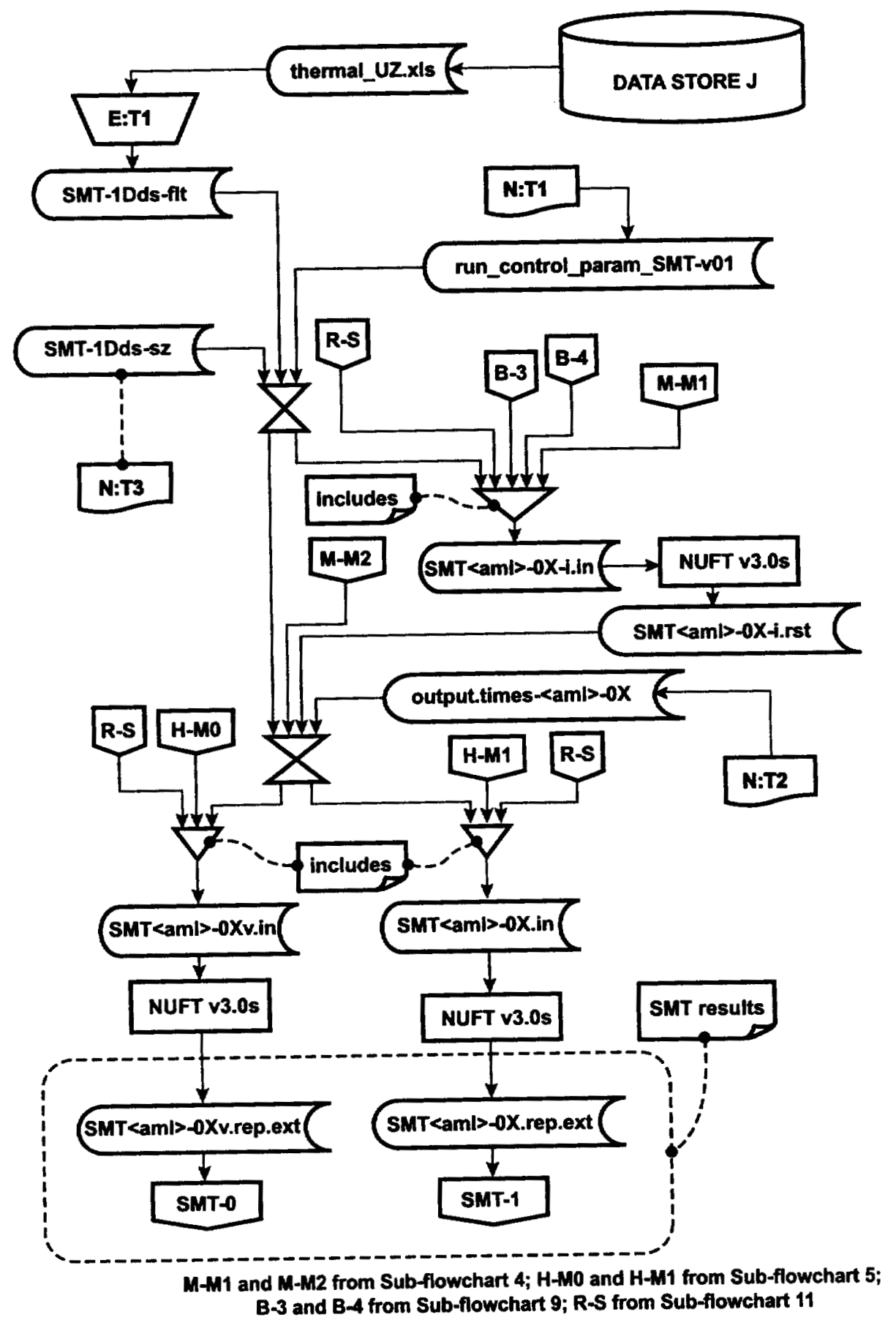

SMT-0 and SMT-1 are EXECUTABLE OUTPUT

Figure 20 Sub-flowchart 16, SMT submodel execution. 


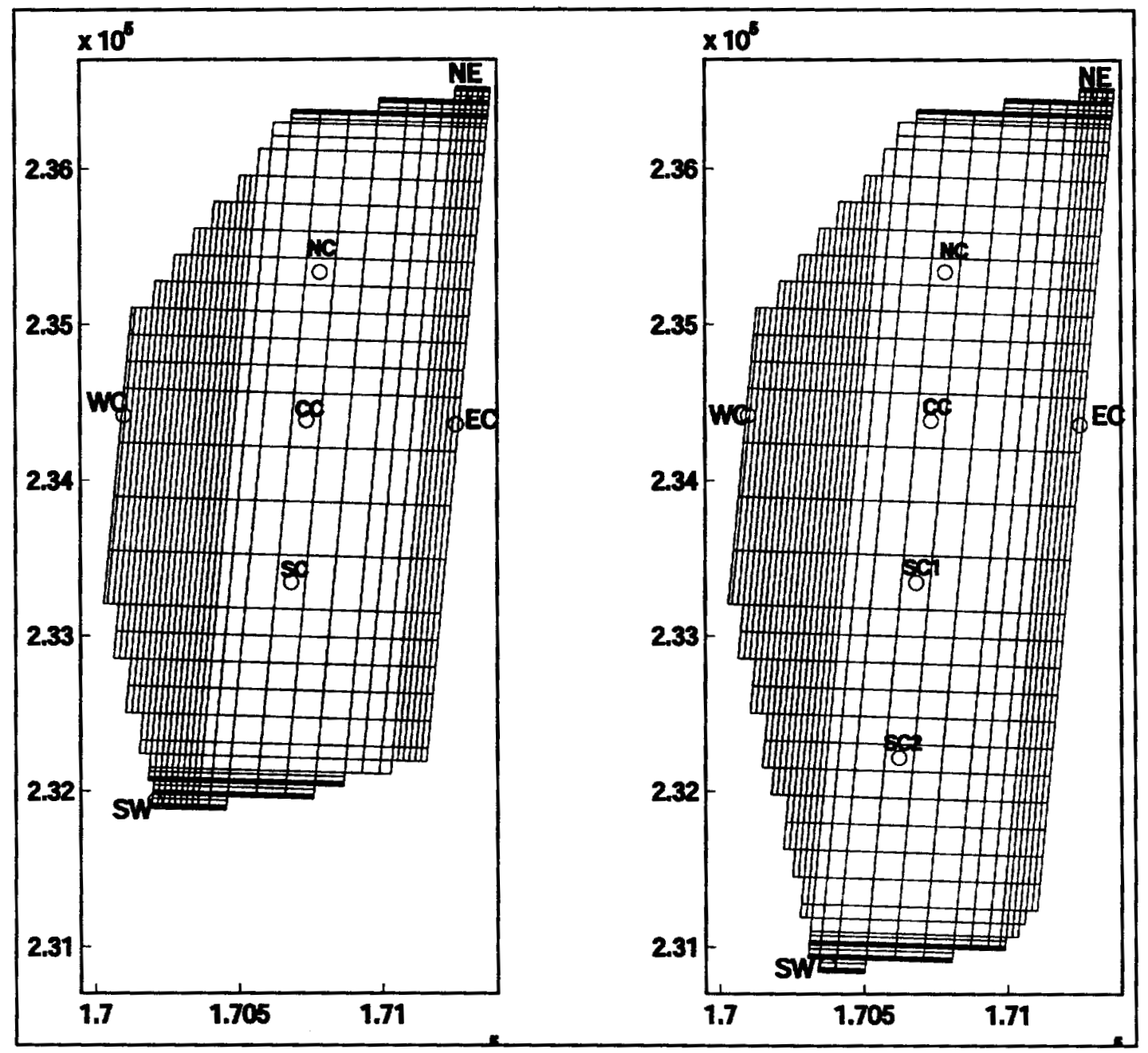

Figure 21 Grid of repository footprint for the HTOM (left) and LTOM (right) operation modes. 


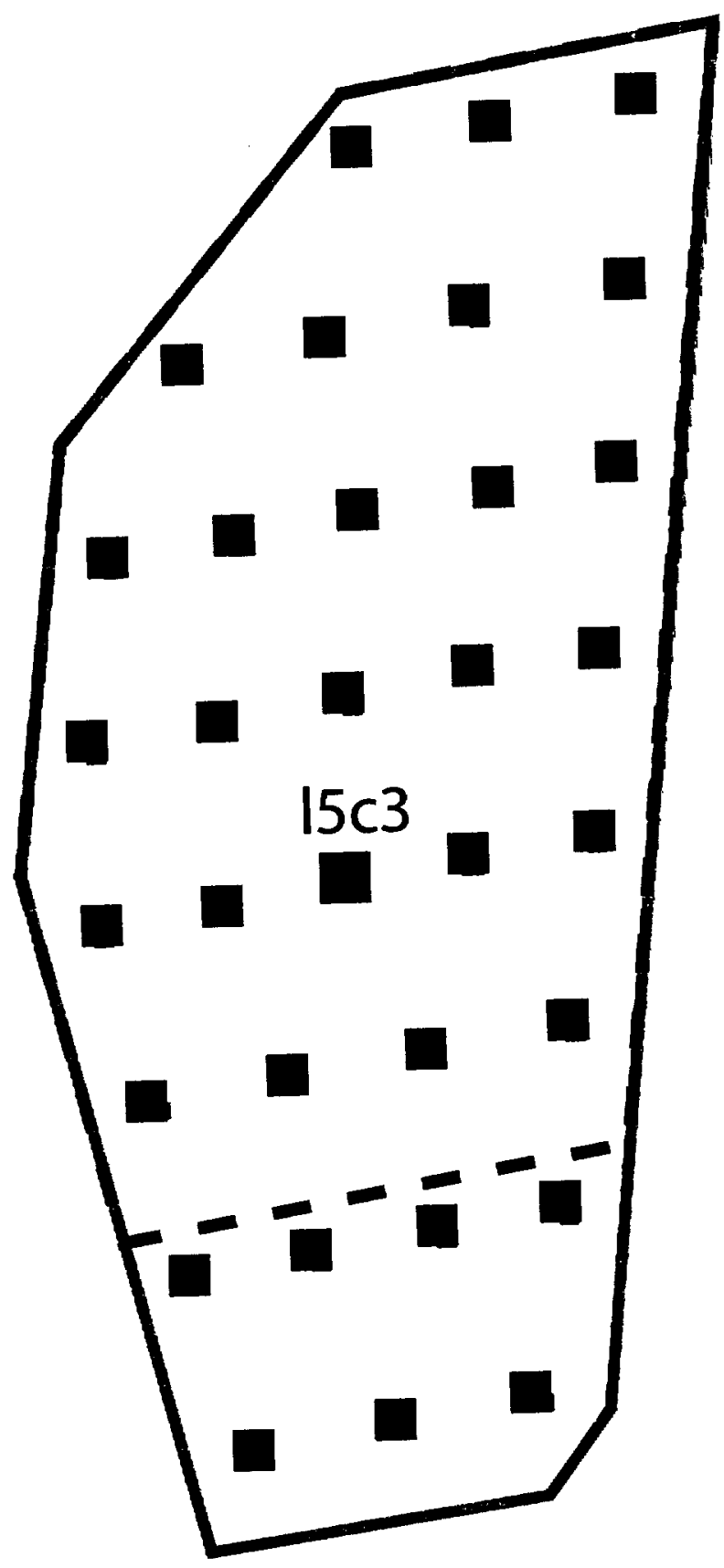

Figure 22 LDTH/SDT calculation locations (total of 33) for the HTOM (red dashed line) and the LTOM (blue solid lines) operation modes. 


\section{Pre-closure LDTH submodel}

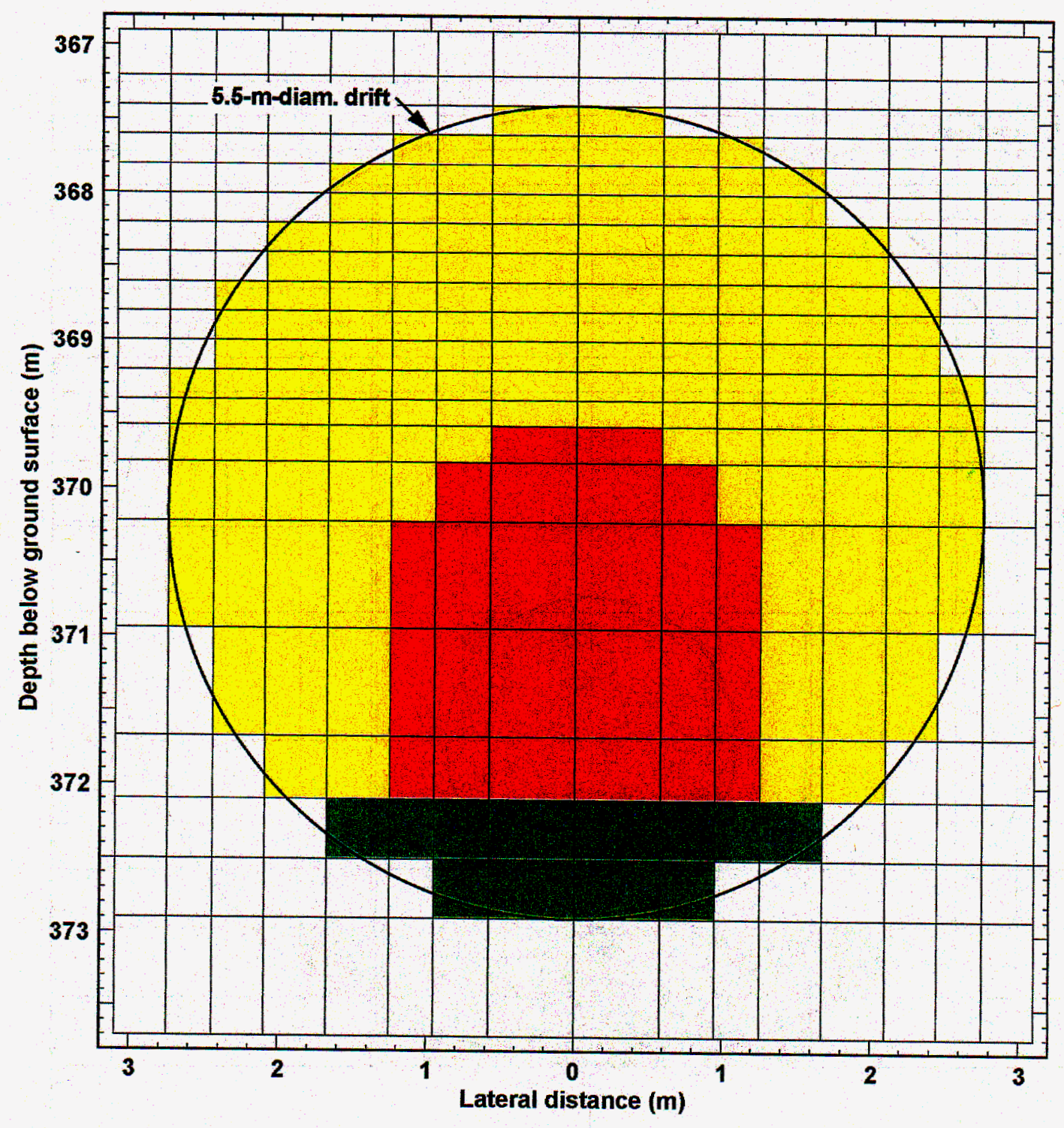

Host rock (tsw 35)

$\square$ Air gap (with effective thermal conductivity representing thermal radiation)

Lumped drip shield, air gap, and waste package

Crushed tuff invert

154 0508.ai

154_0508.ai

Source: Modified from CRWMS M\&O 2000 [DIRS 149862], Figure 6-2, using updated invert dimensions and vertical location for one location in the repository.

NOTE: The values on the vertical axis shift with location within the potential repository footprint.

Figure 23 Numerical Mesh Used in the Line-Source Drift-Scale Thermal-Hydrologic (LDTH) Submodels. 


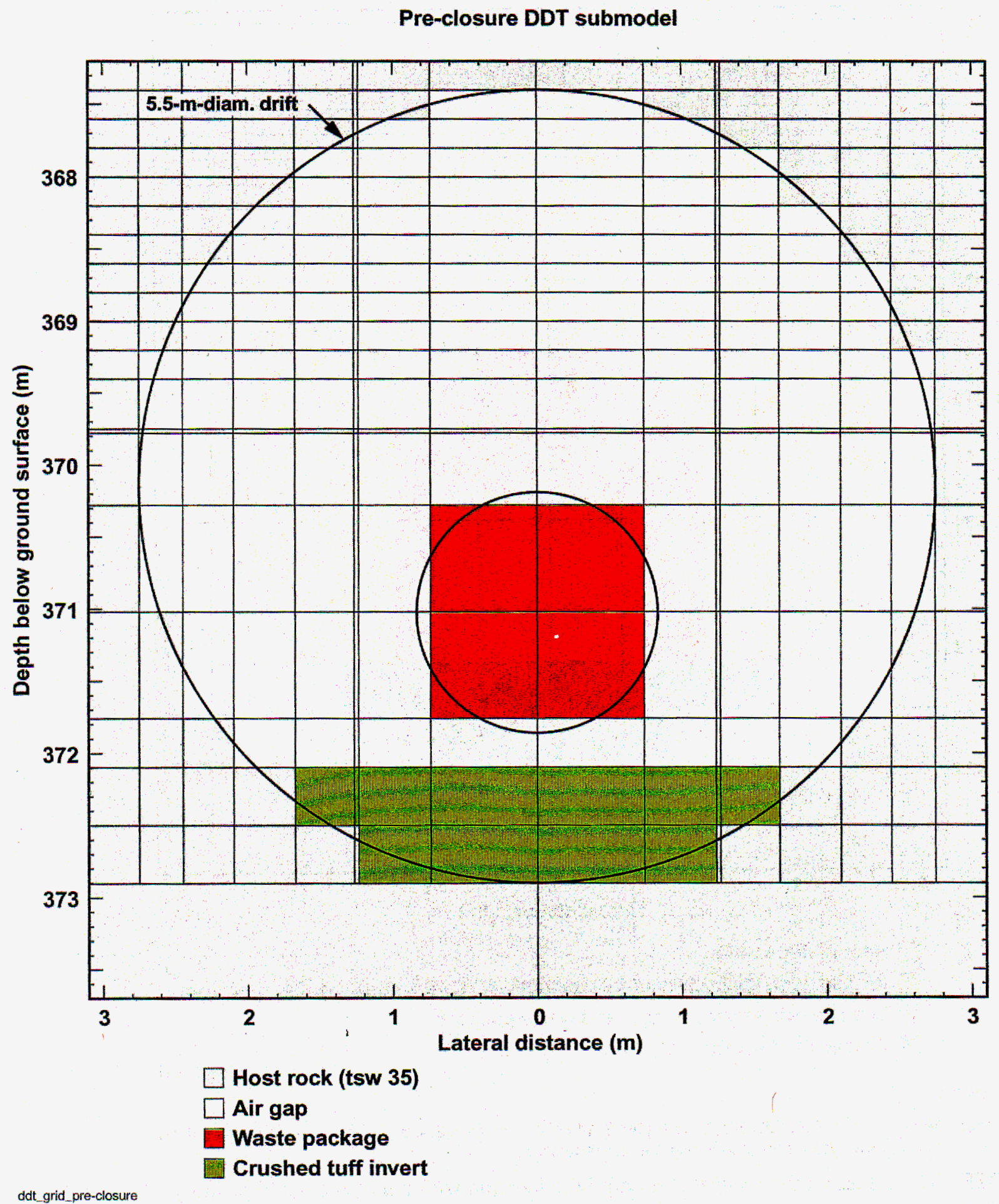

Figure 24 DDT pre-closure mesh. 


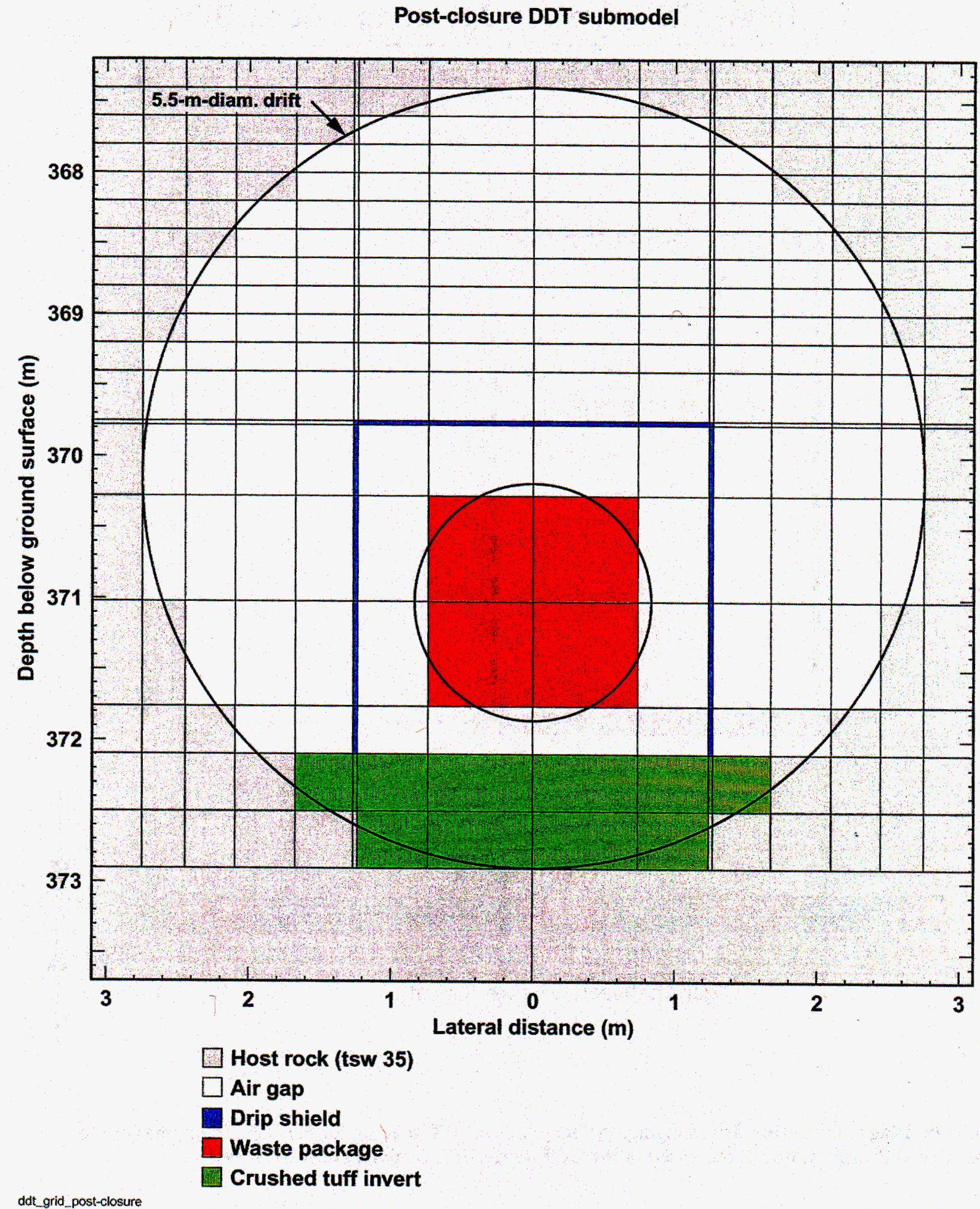

Figure 25 DDT post-closure mesh (includes the drip shield). 


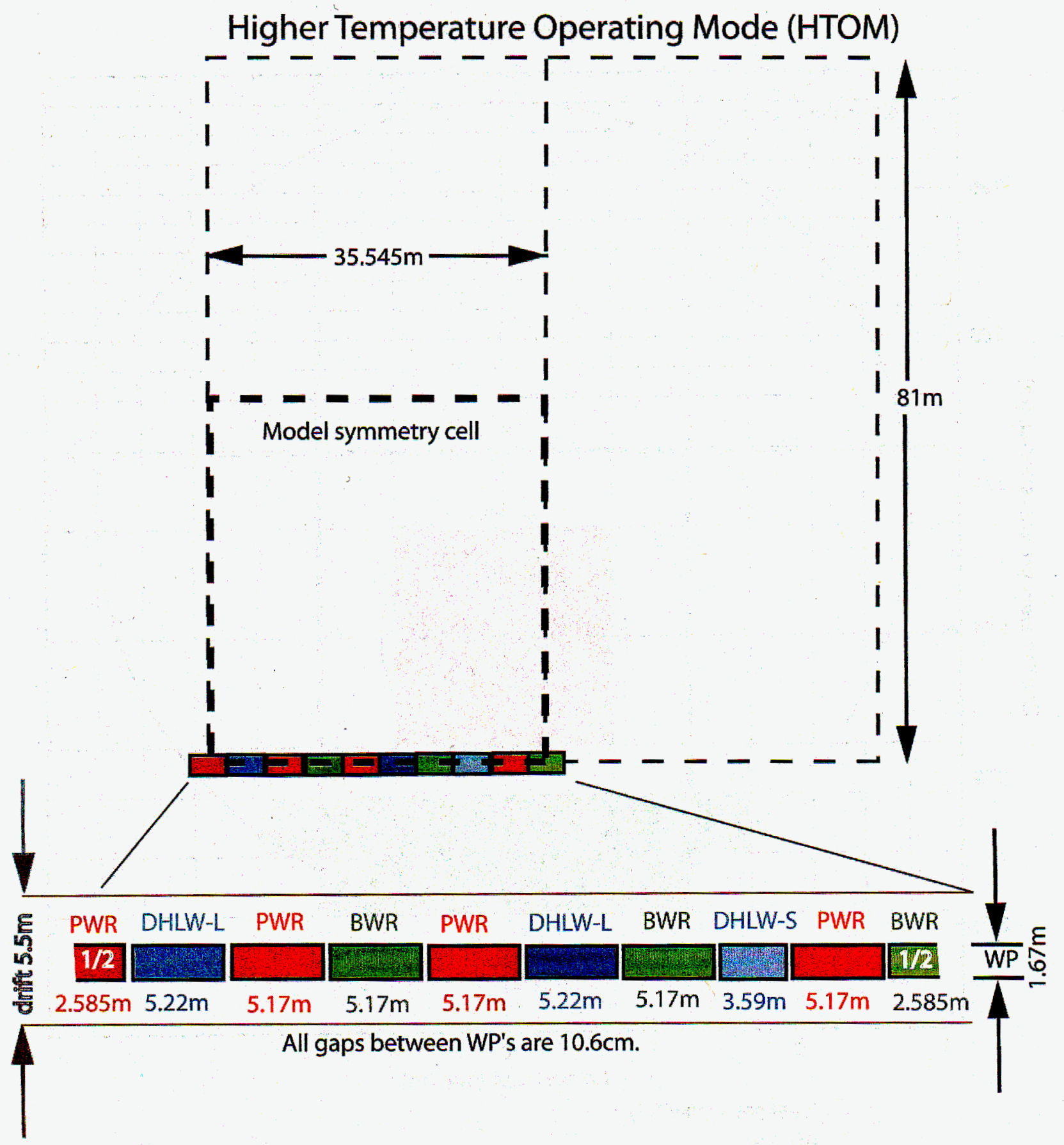

Figure 26 Diagram showing drift spacing, WP lengths, and WP spacing for HTOM. The names of the respective WPs (hlw 1, bwr 1, etc.) used in the DDT submodel are given above each WP. 


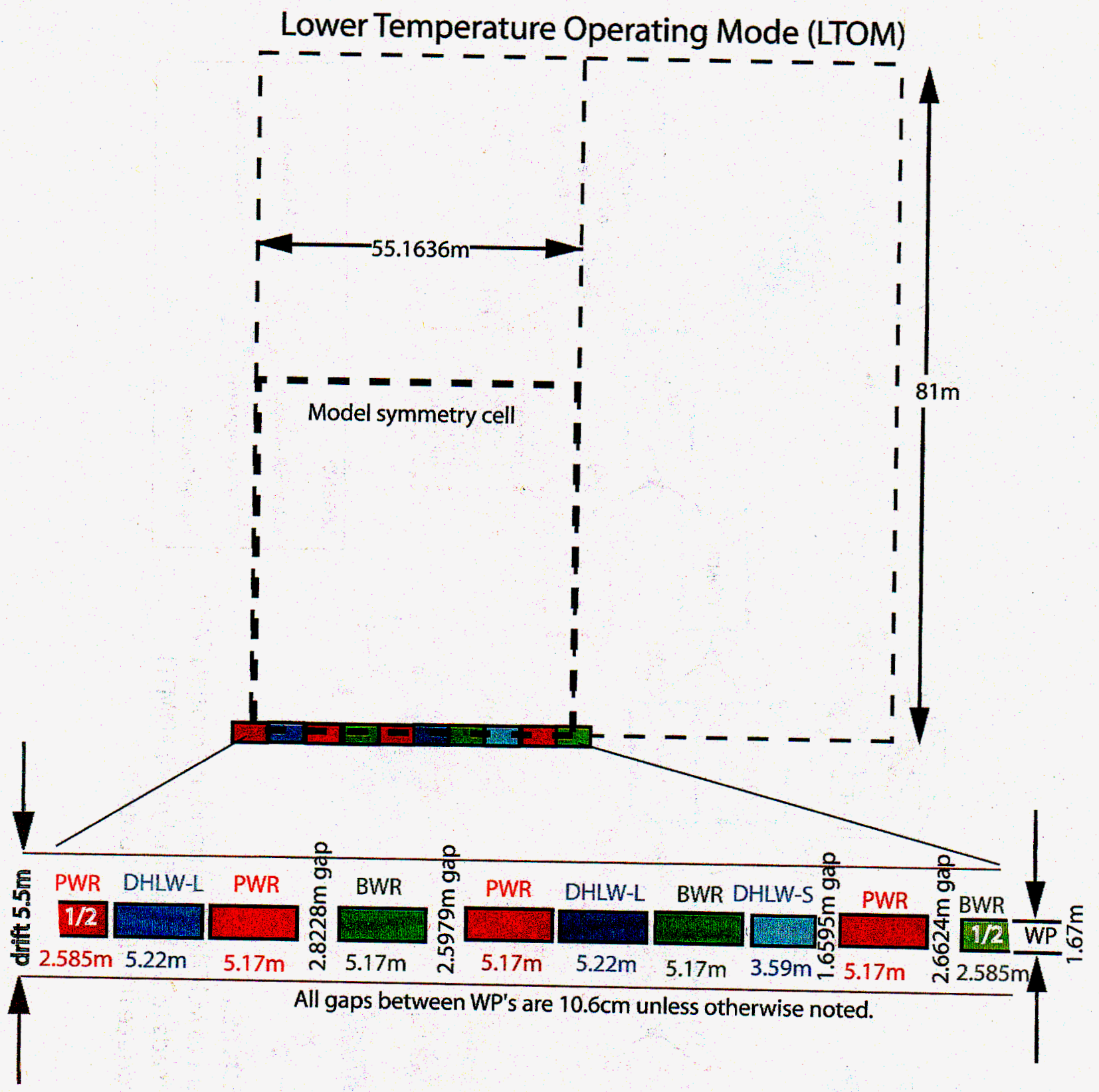

Figure 27 Diagram showing drift spacing, WP lengths, and WP spacing for LTOM. The names of the respective WPs (hlw 1, bwr 1, etc.) used in the DDT submodel are given above each WP. 

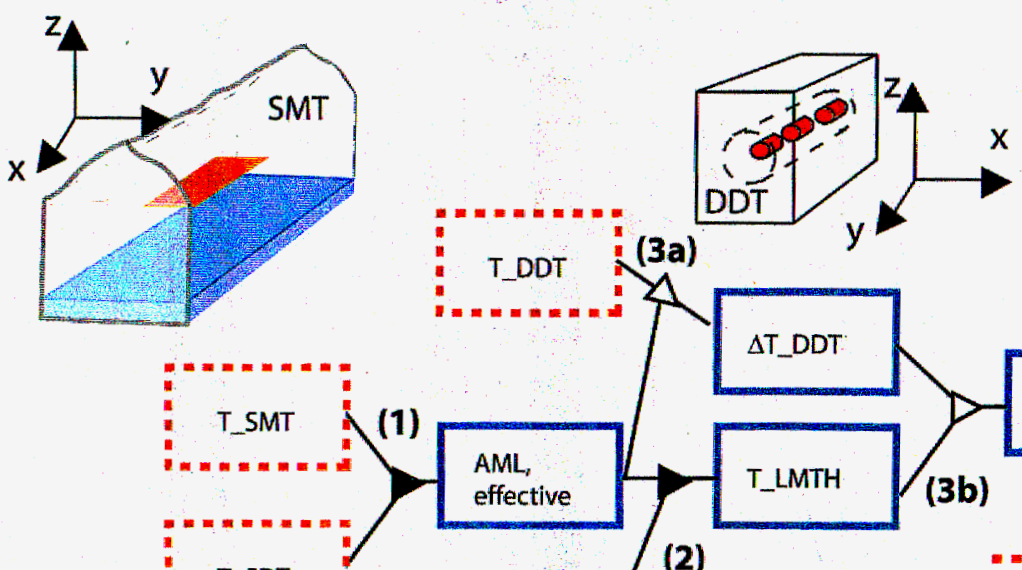

(3b)

T_DMTH
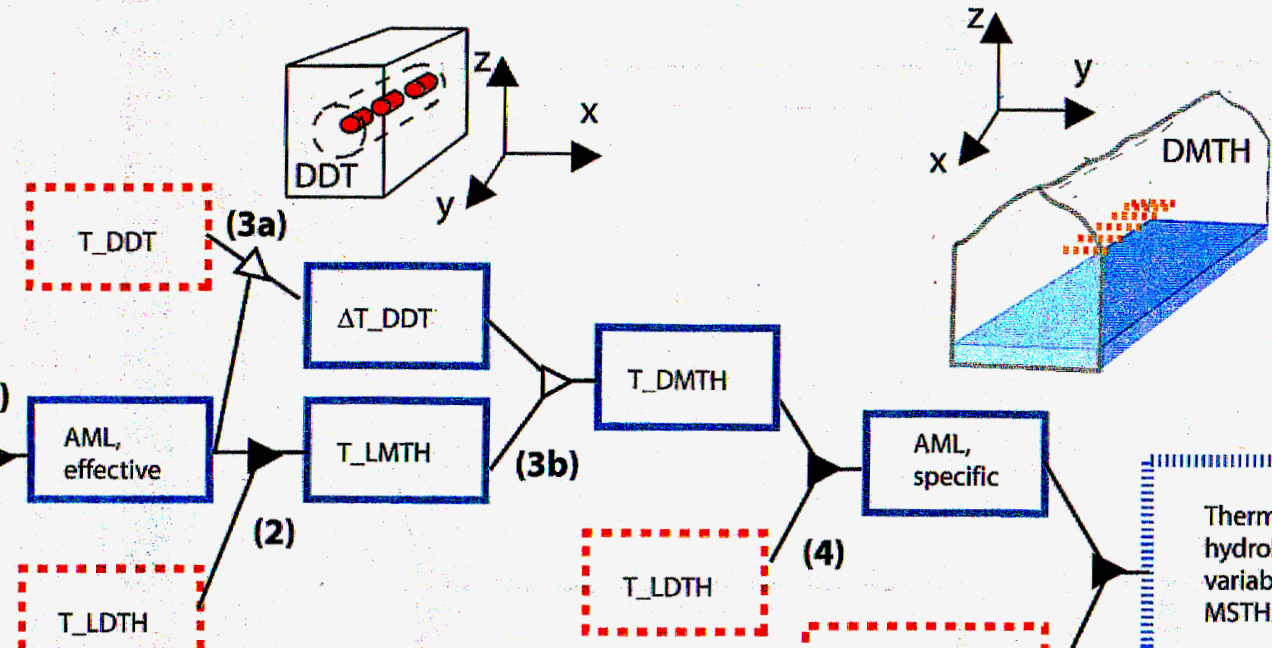

(2)
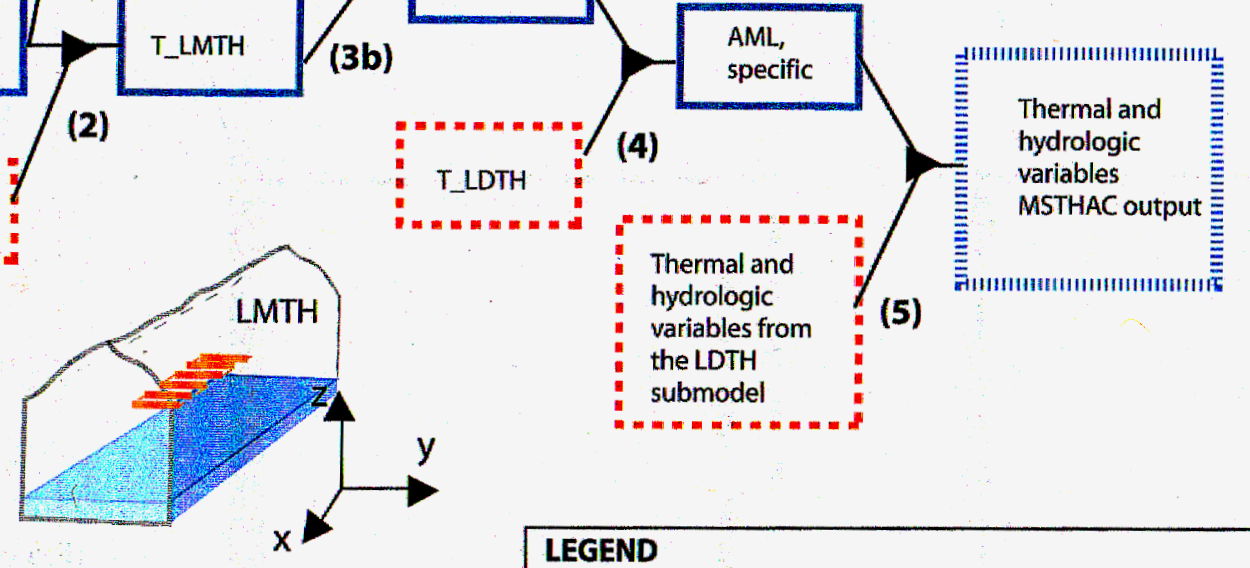

(4)

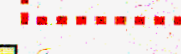

\section{FIVE STEP PROCESS}

STEPS 1 - 2 lead to MSTHAC intermediary model LMTH STEPS 3a-3b leads to MSTHAC final model DMTH STEPS $4-5$ produce the final T-H output

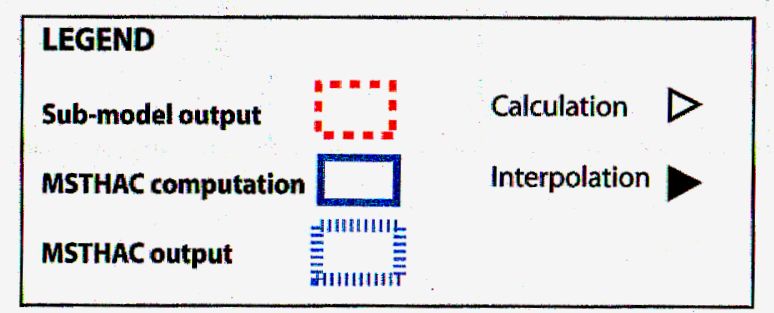


(a) liquid-phase flux $5 \mathrm{~m}$ above drift

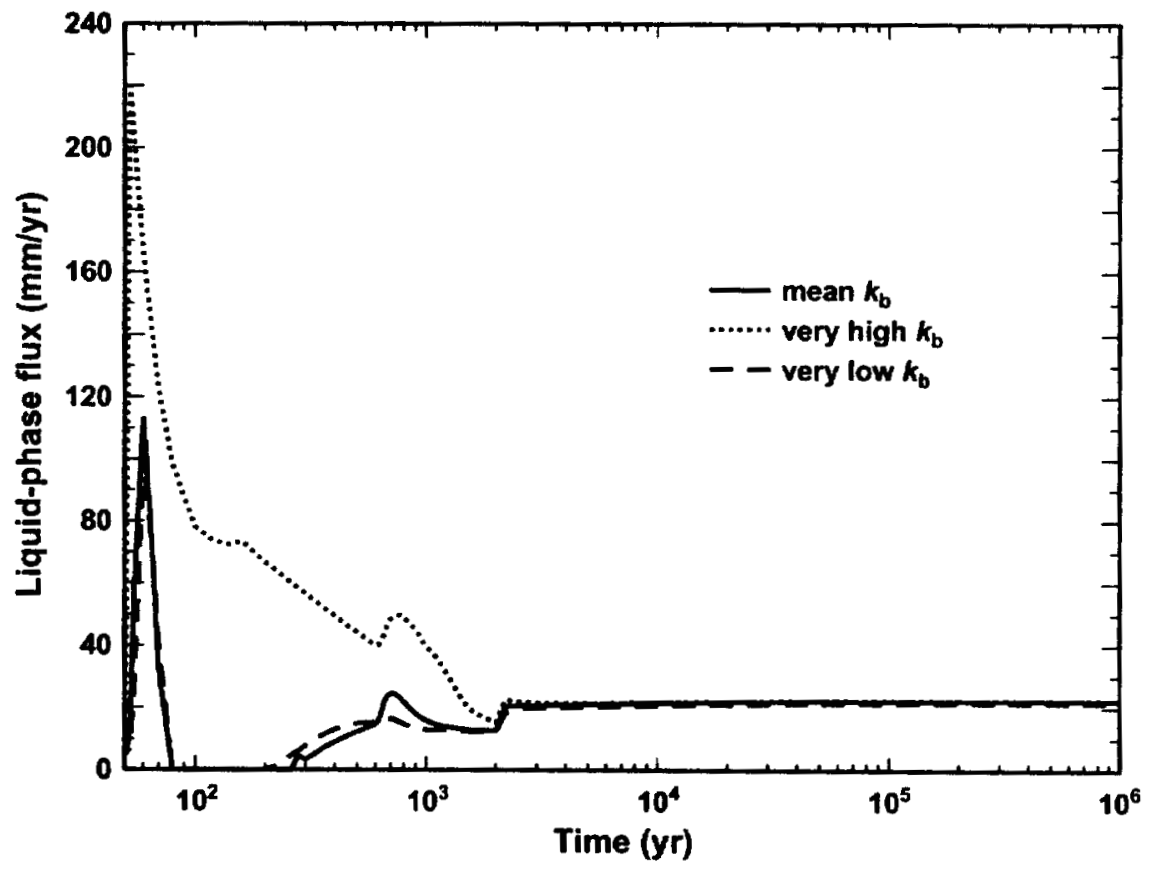

(b) liquid-phase flux $1 \mathrm{~m}$ above drift

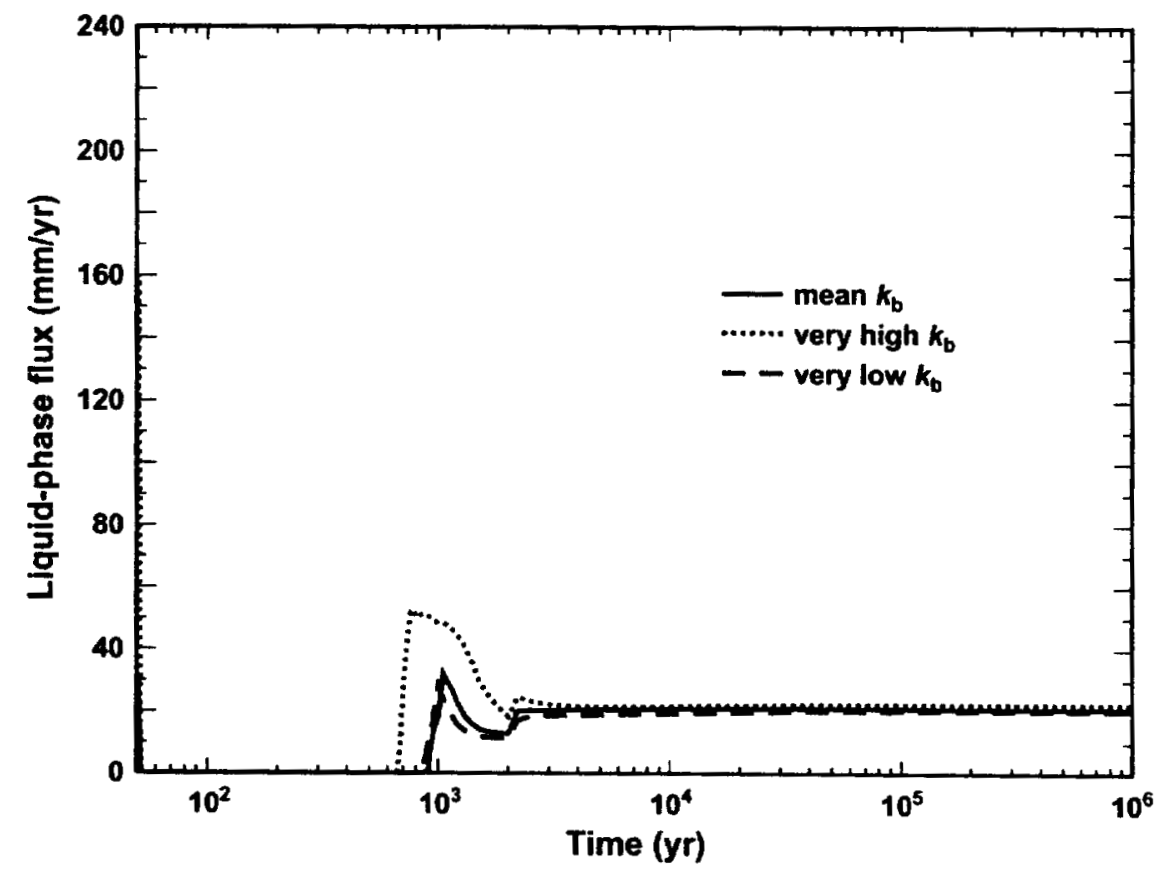

qliq_55_ALLkb

Source: Produced using files from Buscheck 2001 [DIRS 155012].

NOTE: Results for a central location in the repository. $k_{b}=$ bulk permeability.

Figure 29 (a) Liquid phase flux $5 \mathrm{~m}$ above Drift Wall as a function of permeability for HTOM. (b) Liquid phase flux $1 \mathrm{~m}$ above Drift Wall as a function of permeability for HTOM. 
(a) liquid-phase flux $5 \mathrm{~m}$ above drift

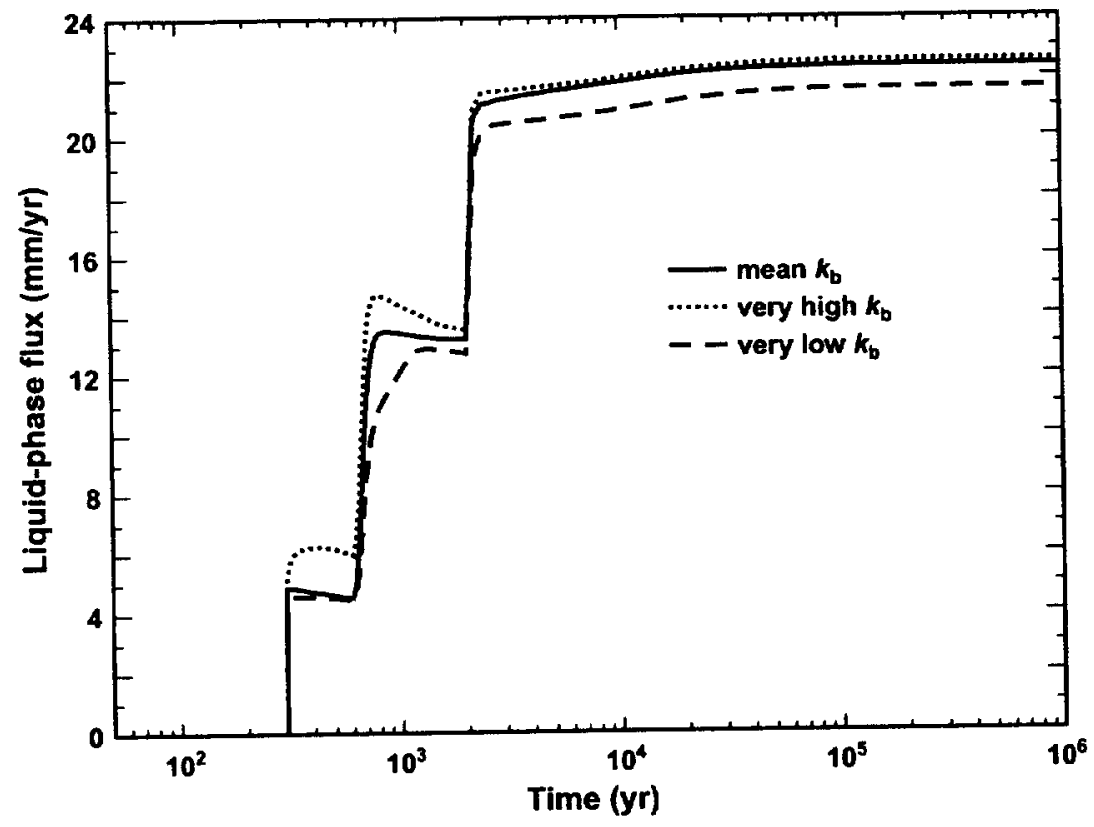

(b) liquid-phase flux $1 \mathrm{~m}$ above drift

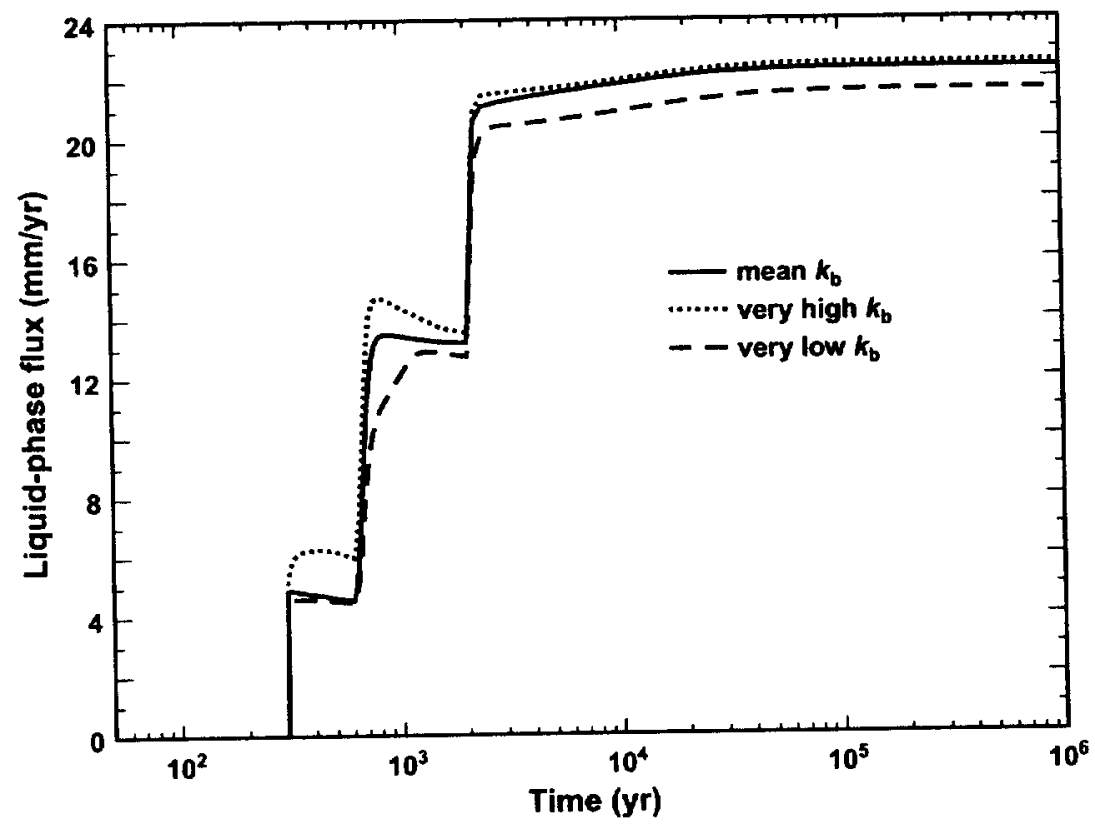

qliq_46_ALLkb

Source: Produced using files from Buscheck 2001 [DIRS 155012].

NOTE: Results for a central location in the repository. $k_{b}=$ bulk permeability.

Figure 30 (a) Liquid phase flux $5 \mathrm{~m}$ above Drift Wall as a function of permeability for LTOM. (b) Liquid phase flux $1 \mathrm{~m}$ above Drift Wall as a function of permeability for LTOM. 
(a) drift wall

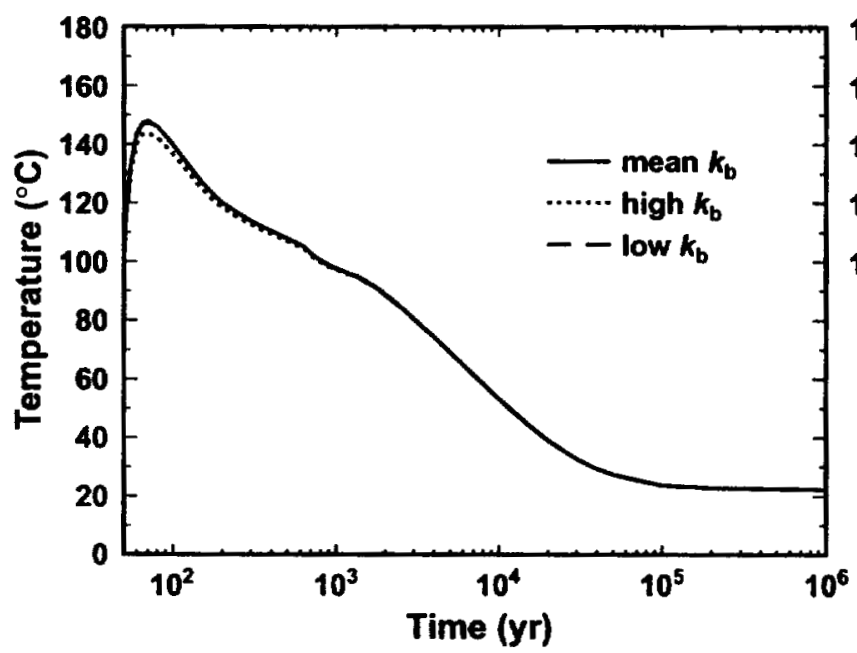

(c) drift wall

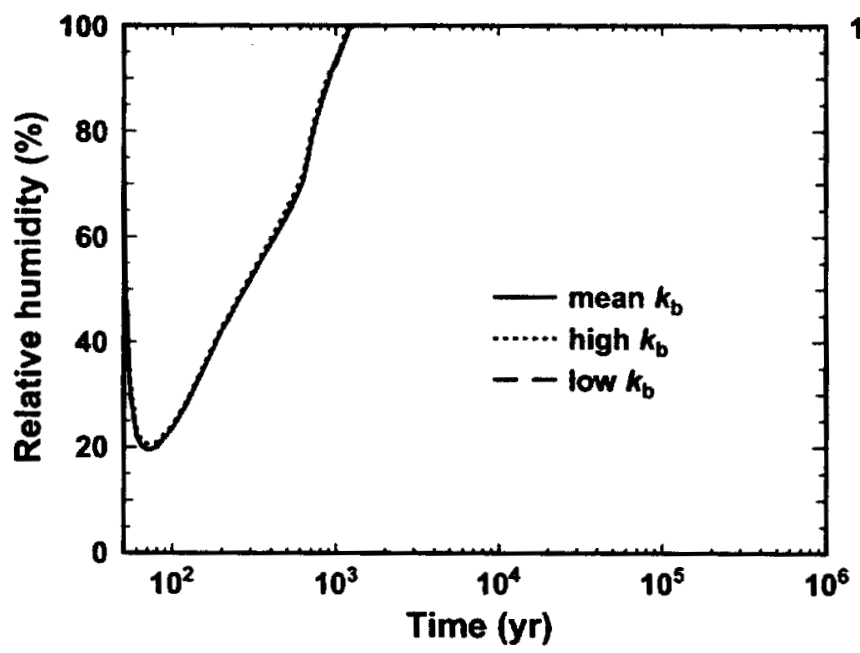

(b) drip shield

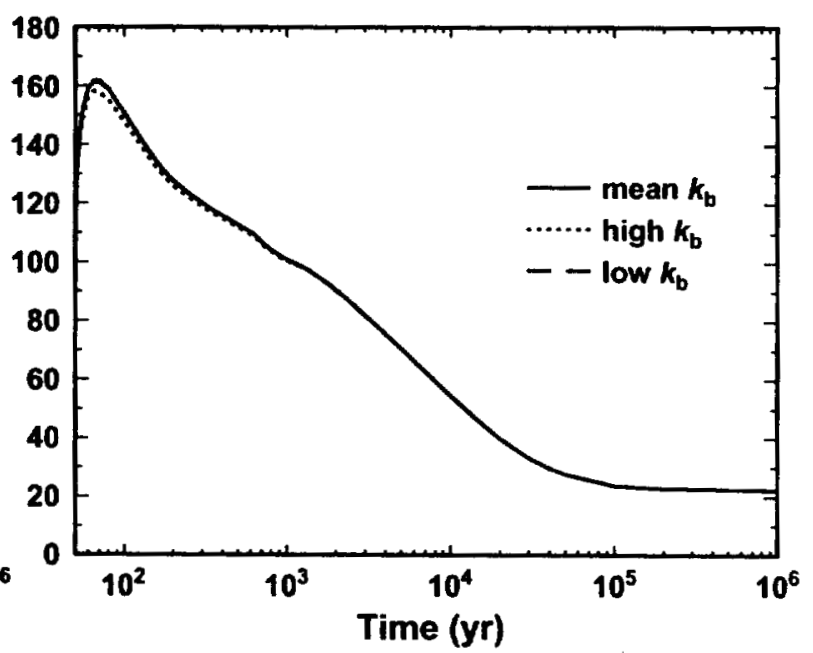

(d) drip shield

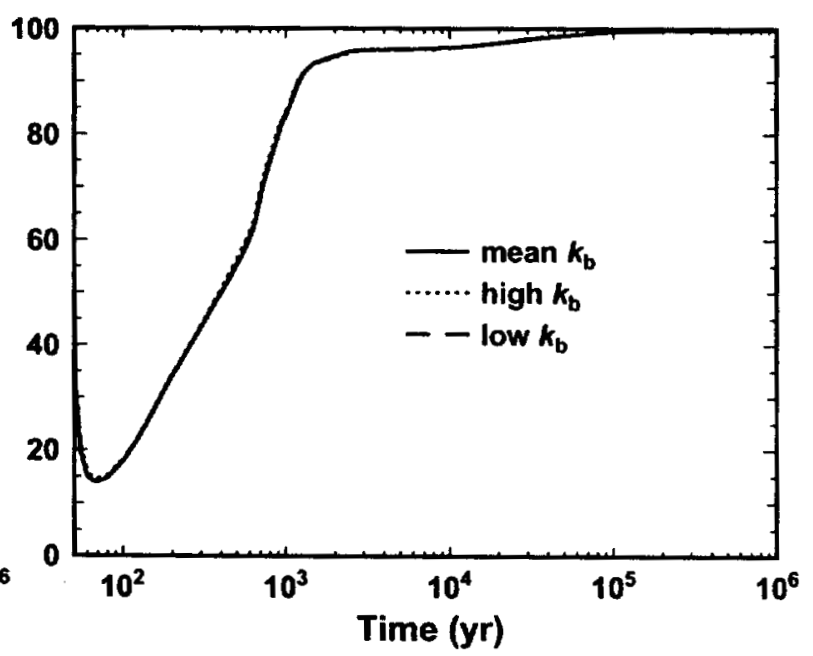

a-d_55_allkb

Source: Produced using files from Buscheck 2001 [DIRS 155012].

NOTE: Higher-temperature operating mode drift wall and drip shield temperatures and relative humidities at the central repository location (Location LSC3) as a function of bulk permeability $\left(k_{b}\right)$ for the mean infiltration-flux scenario.

Figure 31 Drift wall and drip shield temperatures and relative humidities as a function of bulk permeability for HTOM. 
(e) drift wall

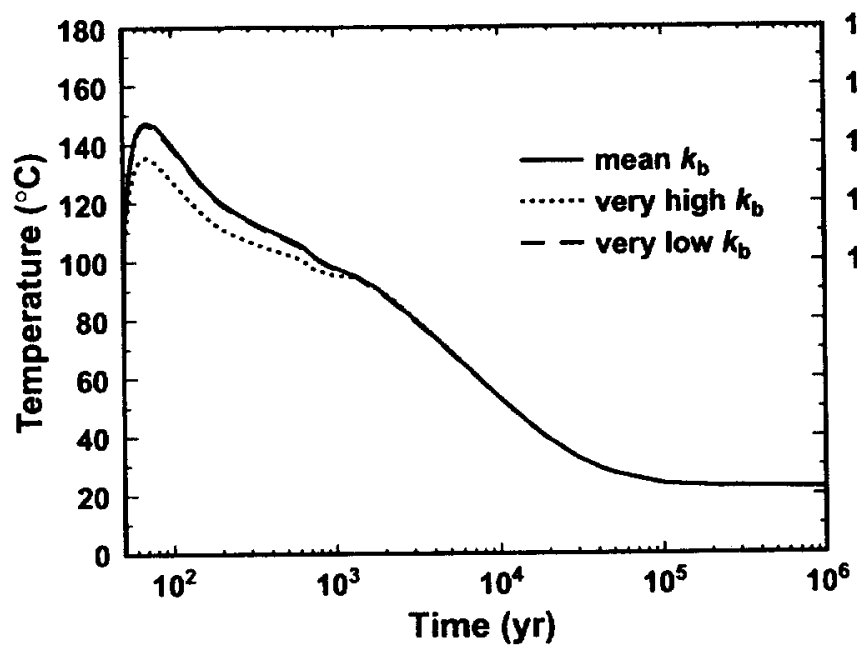

(g) drift wall

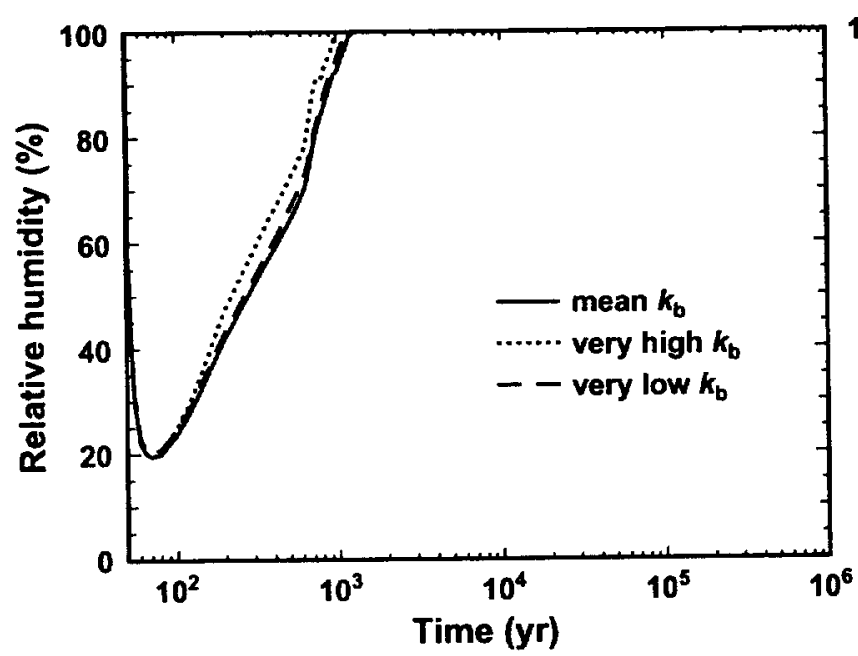

(f) drip shield

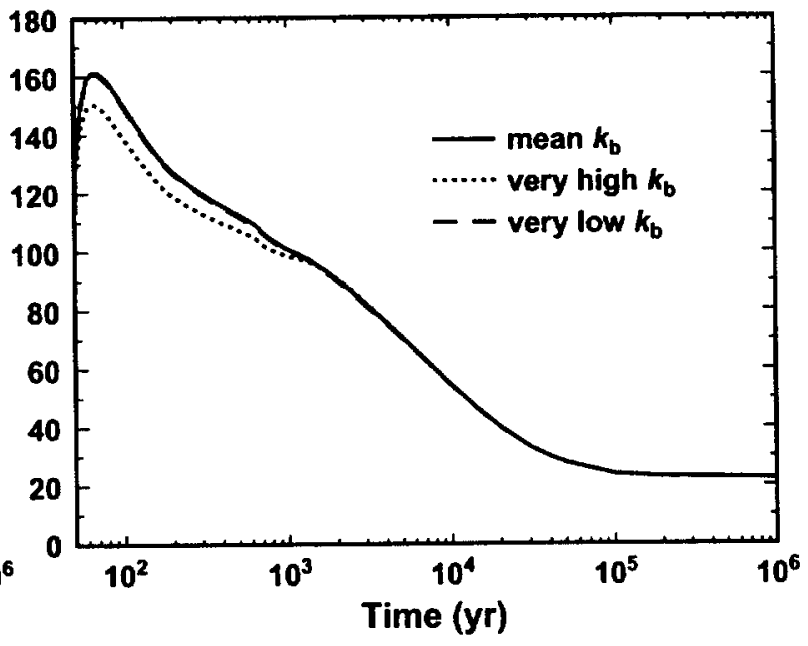

(h) drip shield

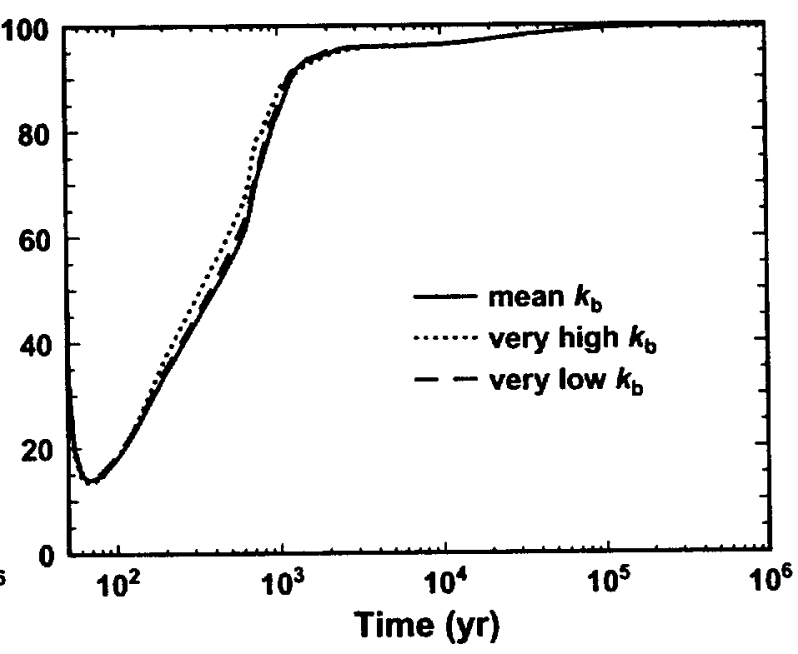

e-h_55_ALLkb

Source: Produced using files from Buscheck 2001 [DIRS 155012].

NOTE: Higher-temperature operating mode drift wall and drip shield temperatures and relative humidities at the central repository location (Location L5C3) as a function of bulk permeability $\left(\mathrm{k}_{\mathrm{b}}\right)$ for the mean infiltration-flux scenario.

Figure 31 Drift wall and drip shield temperatures and relative humidities as a function of bulk permeability for HTOM. 

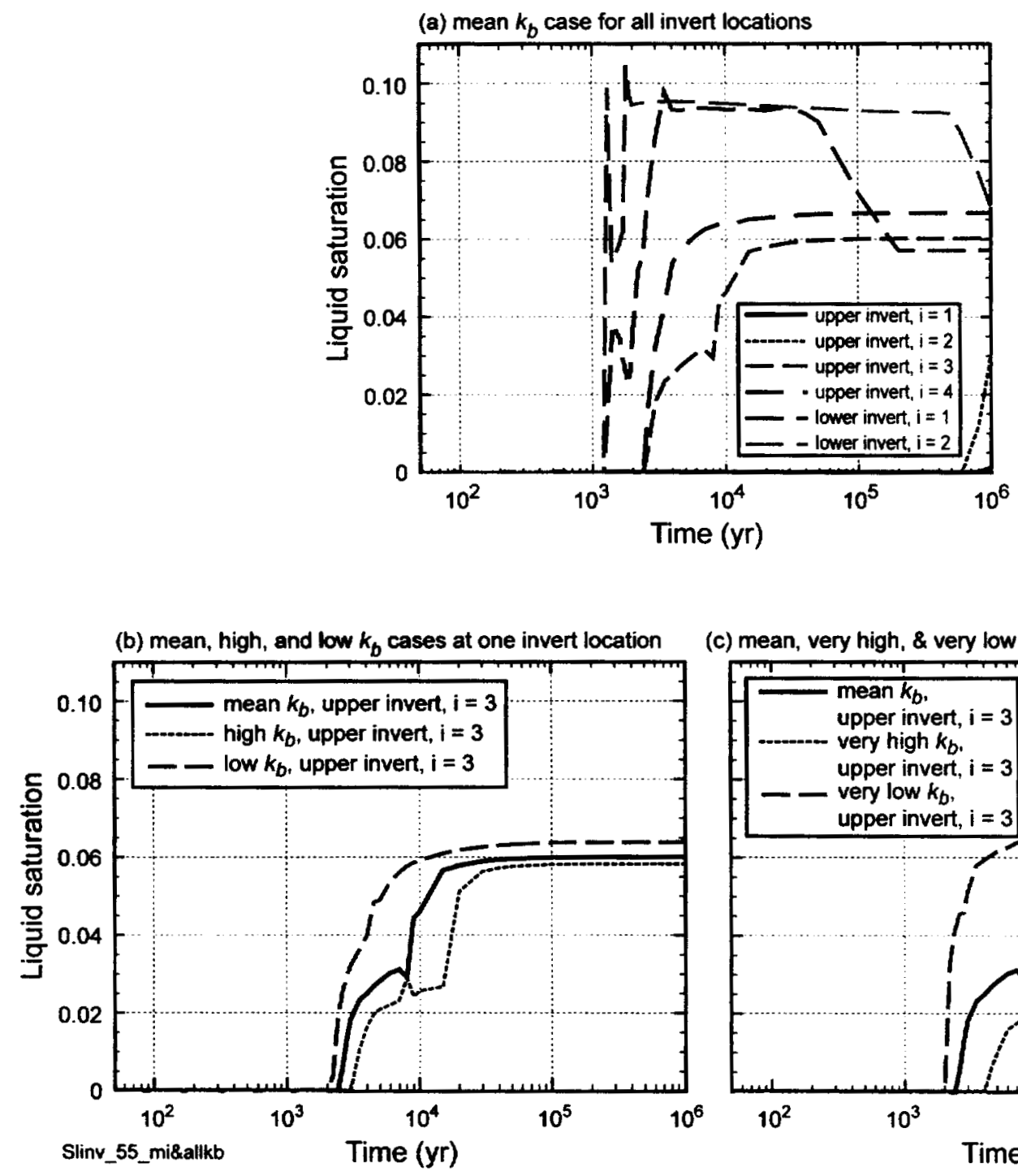

(c) mean, very high, \& very low $k_{b}$ cases at one inver

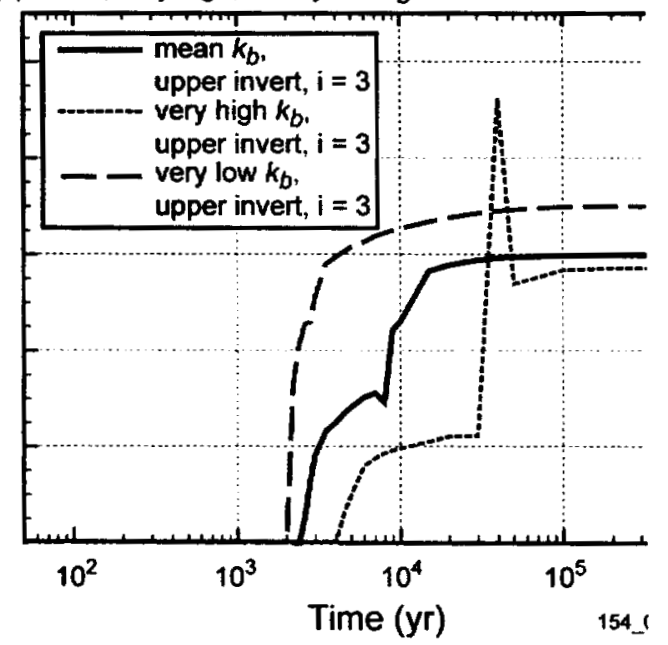

154_0370a.ai

Source: Produced using files from Buscheck 2001 [DIRS 155012].

NOTE:Higher-temperature operating mode invert liquid saturations at the central repository location (Location $\mathrm{L} 5 \mathrm{C} 3$ ) as a function of bulk permeability $\left(k_{\mathrm{b}}\right)$ for the mean infiltration-flux scenario.

Figure 32 Invert liquid saturations as a function of bulk permeability for HTOM. 
(a) drift wall

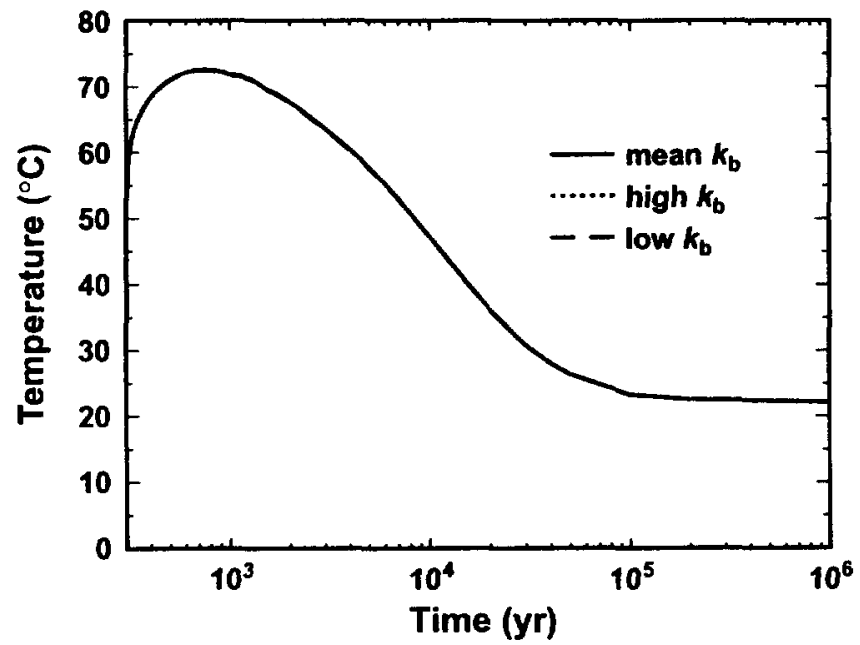

(c) drift wall

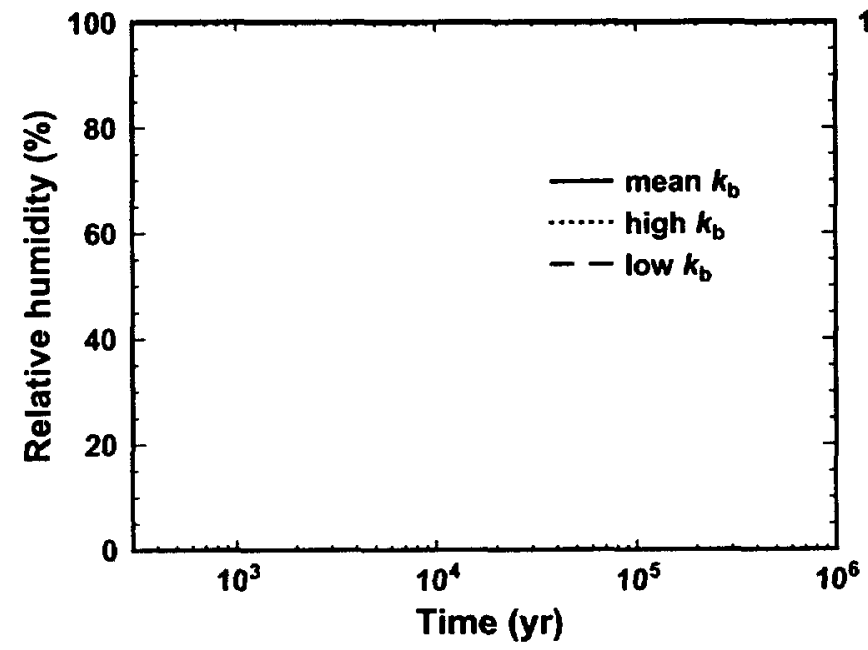

(b) drip shield

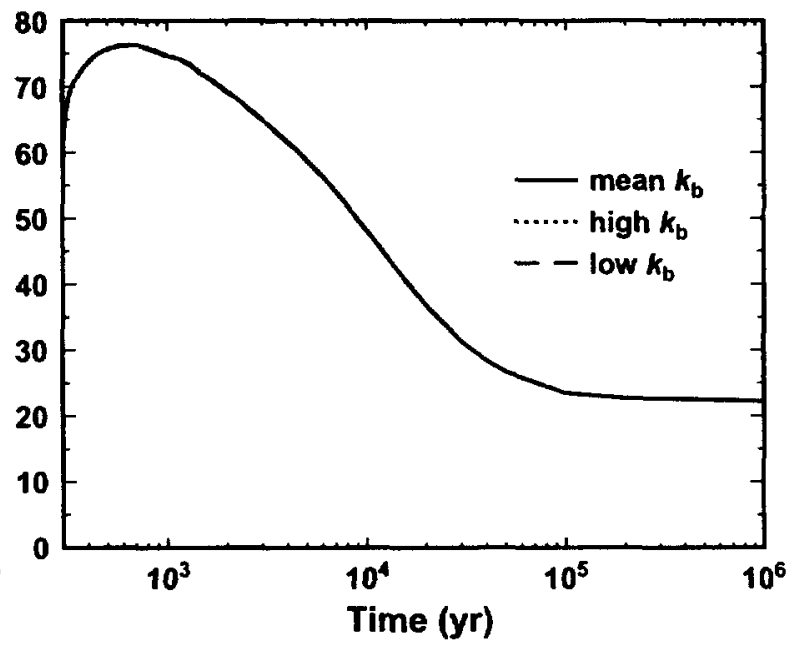

(d) drip shield

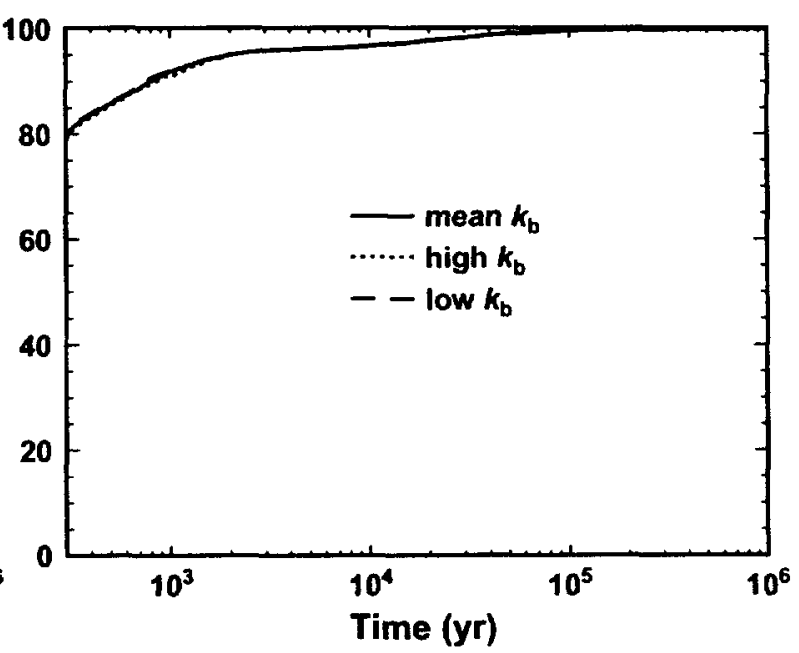

a-d_46_allkb

Source: Produced using files from Buscheck 2001 [DIRS 155012].

NOTE: Lower-temperature operating mode drift wall and drip shield temperatures and relative humidities at the central repository location (Location LSC3) as a function of bulk permeability $\left(k_{\mathrm{b}}\right)$ for the mean infiltration-flux scenario.

Figure 33 Drift wall and drip shield temperatures and relative humidities as a function of bulk permeability for LTOM. 
(e) drift wall

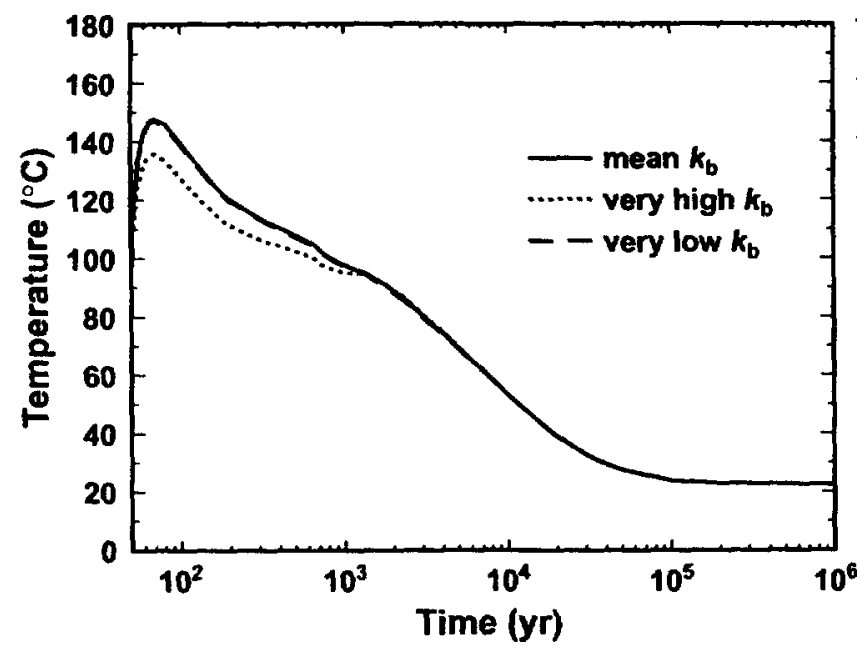

(g) drift wall

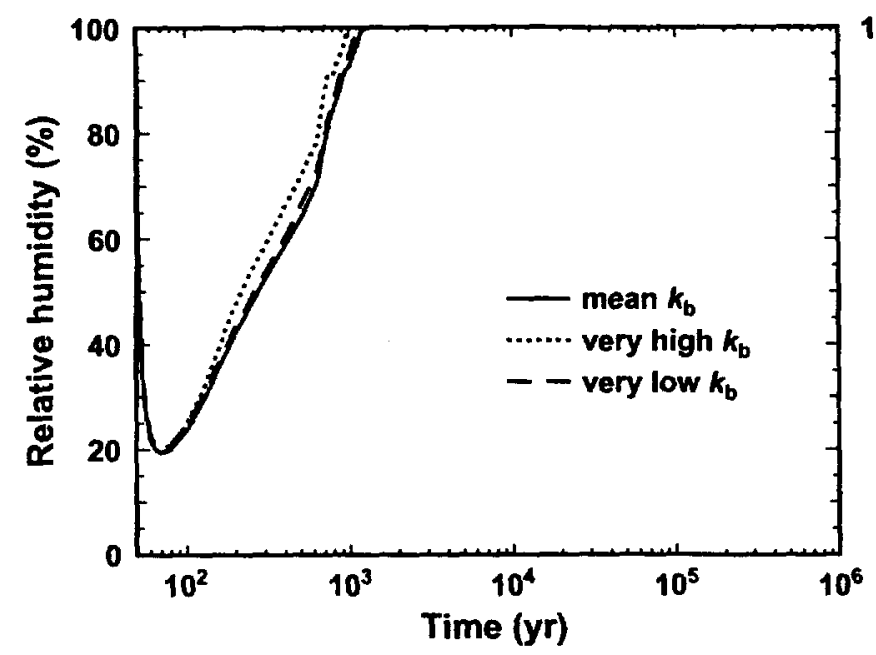

(f) drip shield

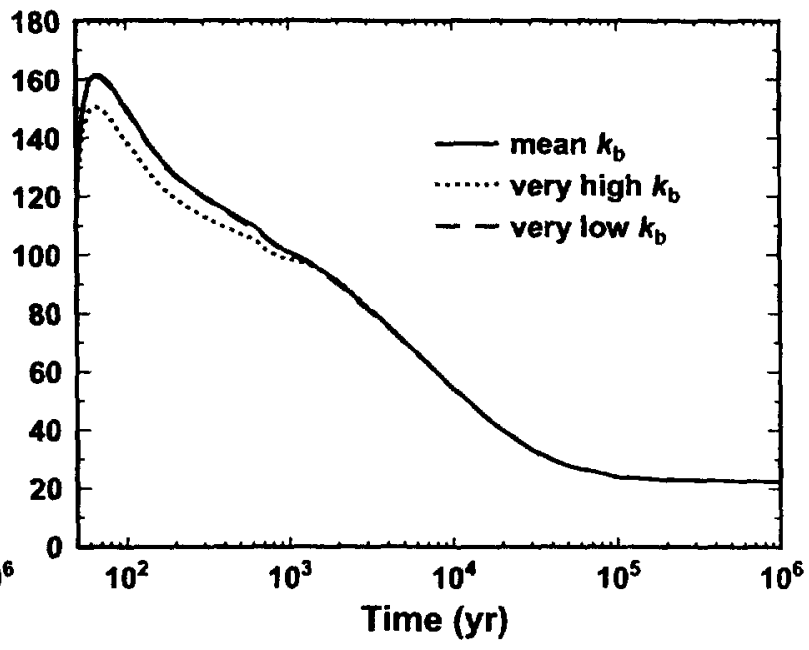

(h) drip shield

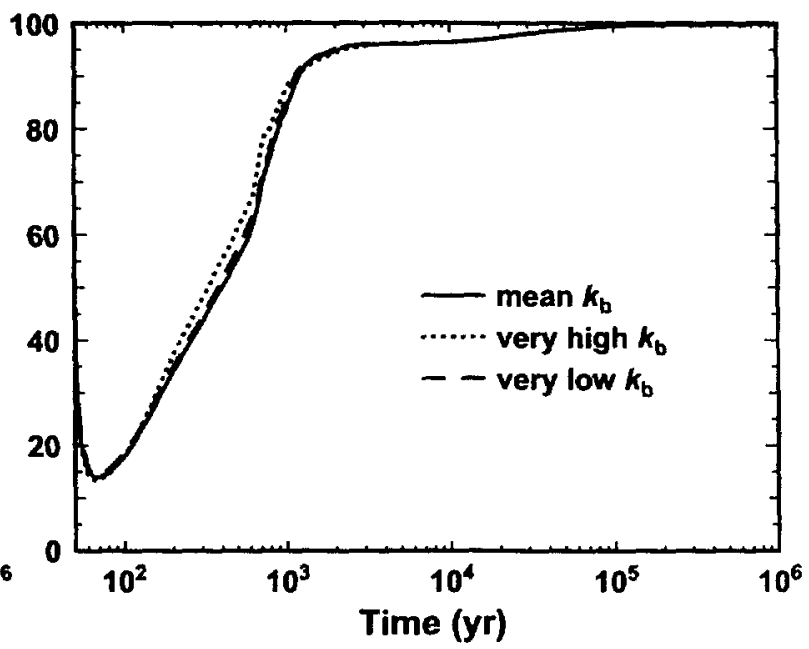

e-h_55_ALLkb

Source: Produced using files from Buscheck 2001 [DIRS 155012].

NOTE: Lower-temperature operating mode drift wall and drip shield temperatures and relative humidities at the central repository location (Location L5C3) as a function of bulk permeability $\left(k_{b}\right)$ for the mean infiltration-flux scenario.

Figure 33 Drift wall and drip shield temperatures and relative humidities as a function of bulk permeability for LTOM. 
(a) mean, high, and low $k_{b}$ cases at one invert location

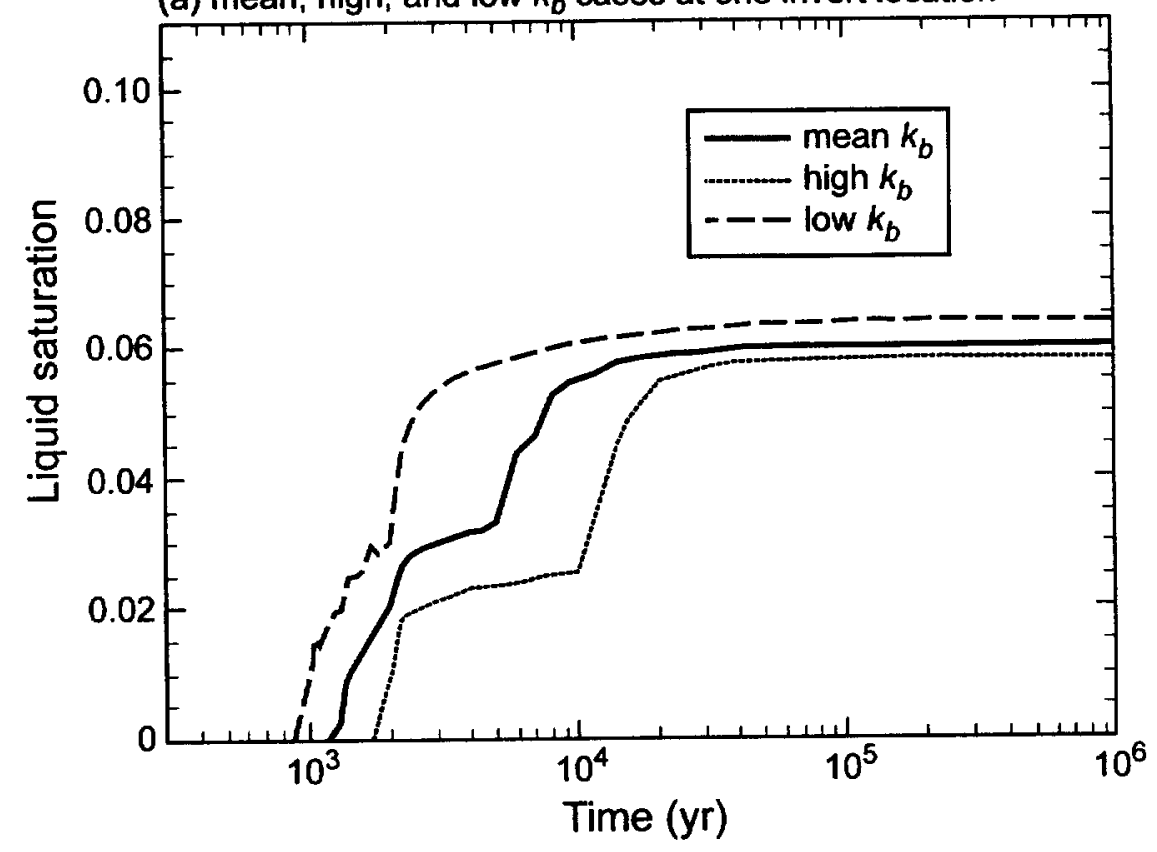

(b) mean, very high, and very low $k_{b}$ cases at one invert location

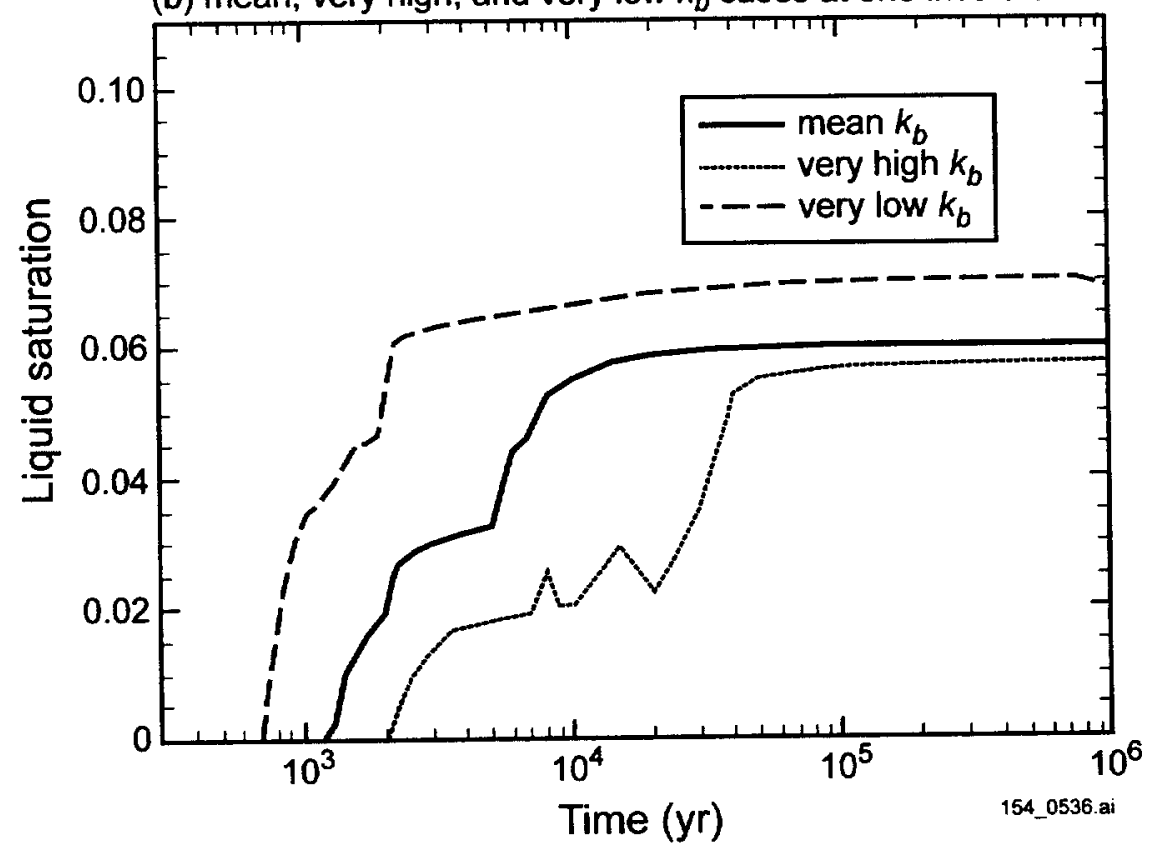

154_0536.ai

Source: Produced using files from Buscheck 2001 [DIRS 155012].

NOTE:Lower-temperature operating mode invert liquid saturations at the central repository location (Location L5C3) as a function of bulk permeability $\left(k_{\mathrm{b}}\right)$ for the mean infiltration-flux scenario.

Figure 34 Invert liquid-saturations as a function of bulk permeability for LTOM. 
(a) drift wall

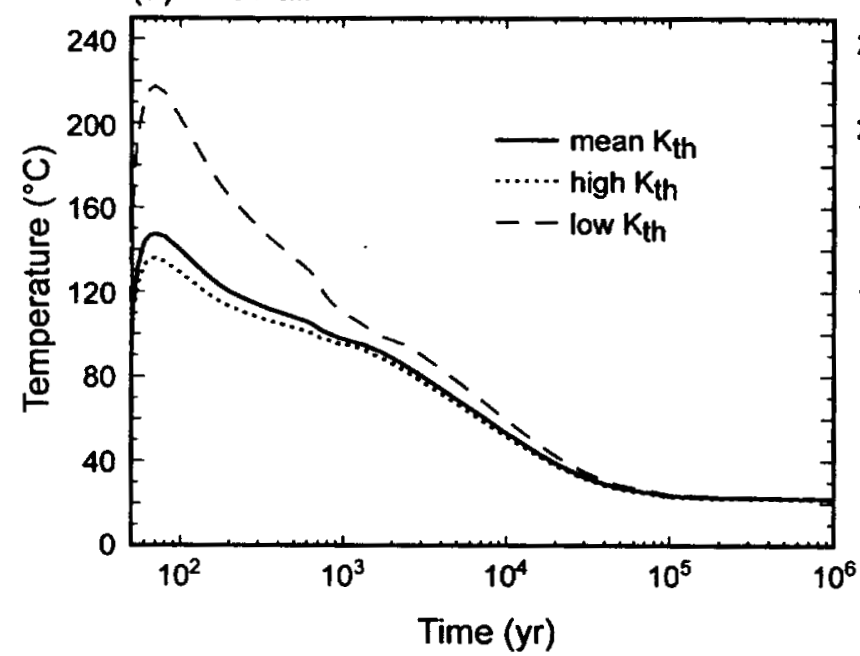

(c) drift wall

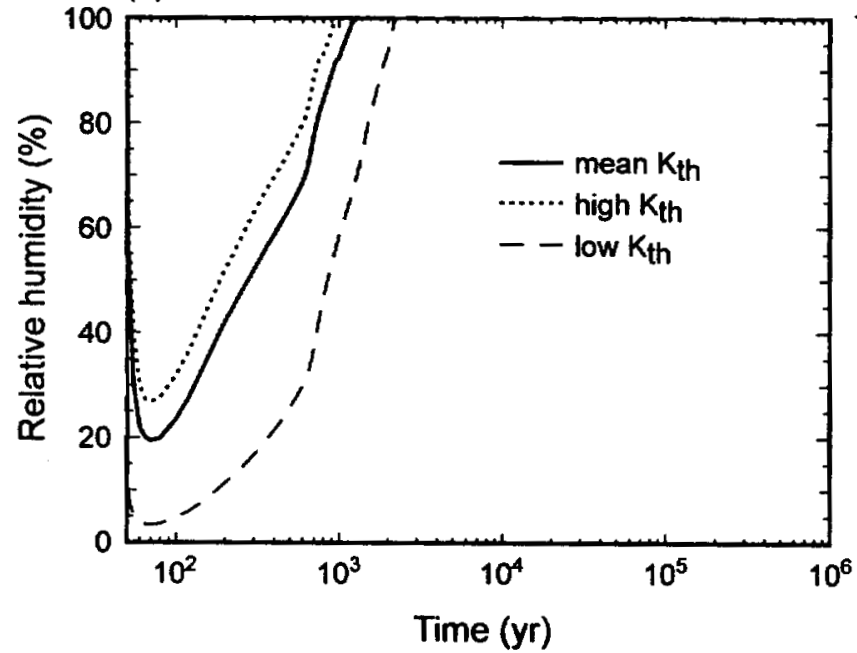

55 allkth (b) drip shield

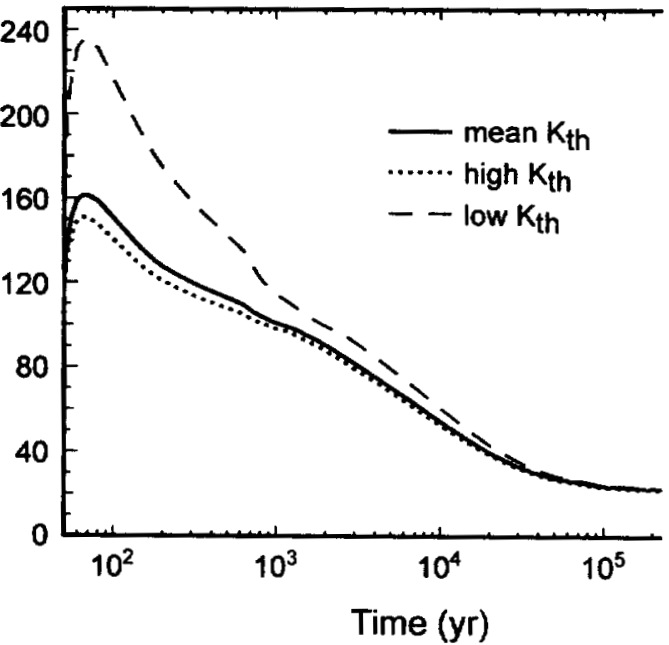

(d) drip shield

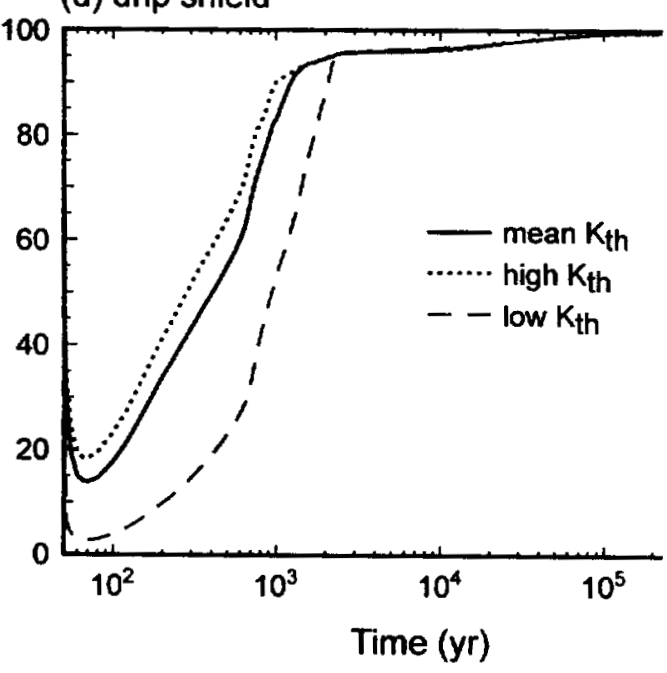

154_0500.ai

Source: Produced using files from Buscheck 2001 [DIRS 155012].

Figure 35 Temperature and relative humidity at the drift wall and drip shield as a function of host-rock thermal conductivity for the central repository (15c3, see Figure 22) location of HTOM and the mean infiltration-flux scenario. 


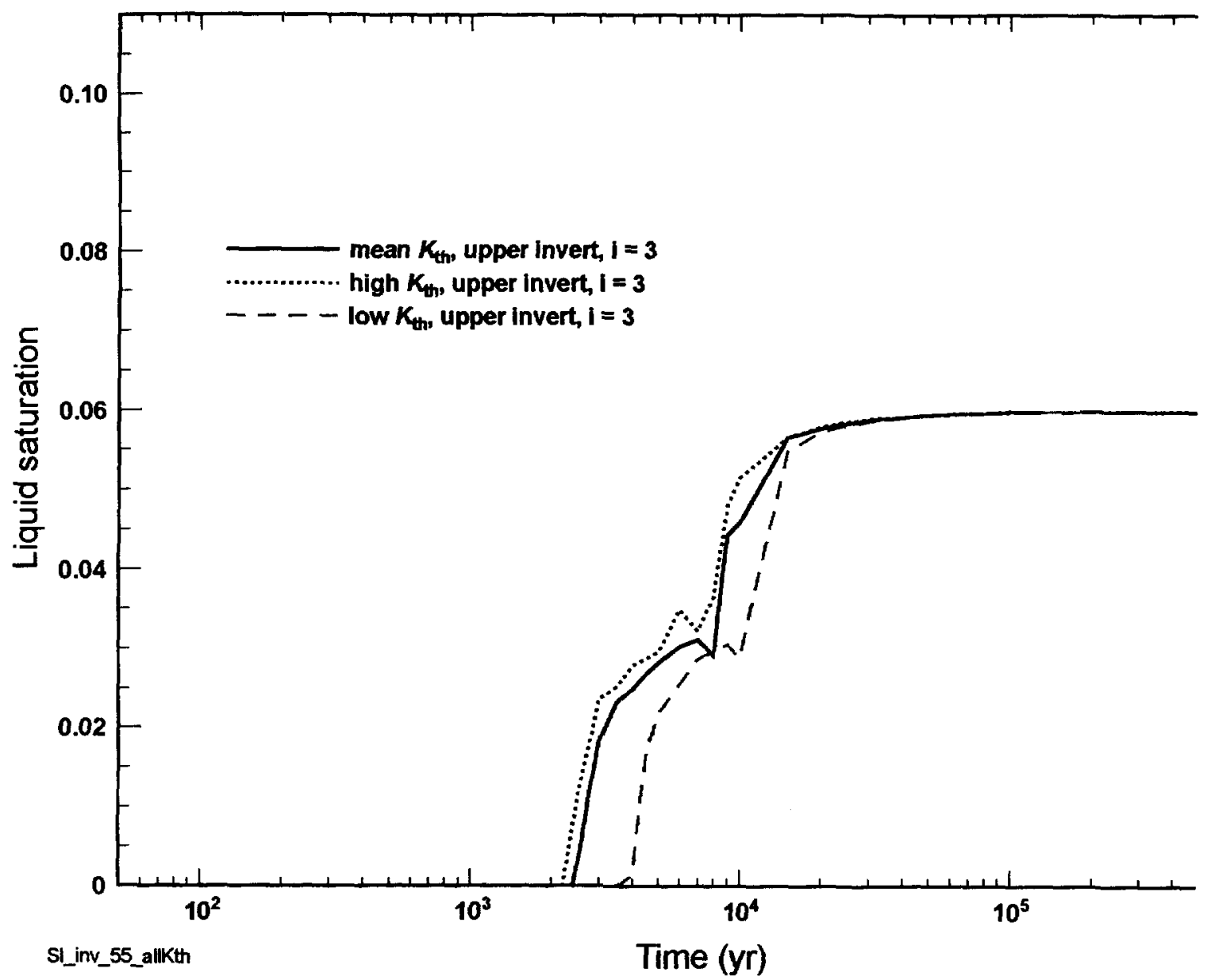

154_0366.ai

Source: Produced using files from Buscheck 2001 [DIRS 155012].

Figure 36 Liquid-Saturation in the Invert as a Function of Host-Rock Thermal Conductivity for the Central Repository (L5C3) Location of HTOM and the Mean Infiltration-Flux Scenario. 
(a) drift wall

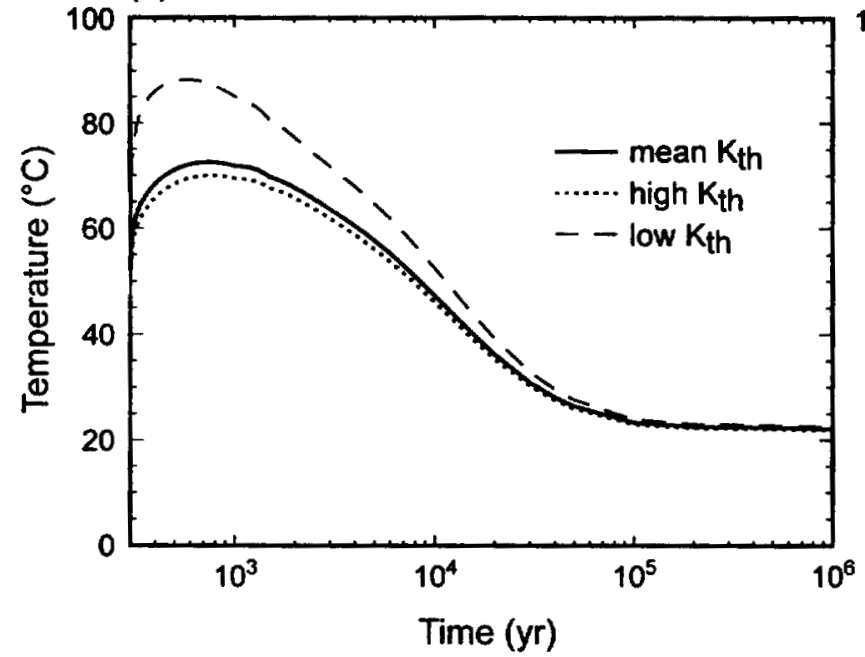

(c) drift wall

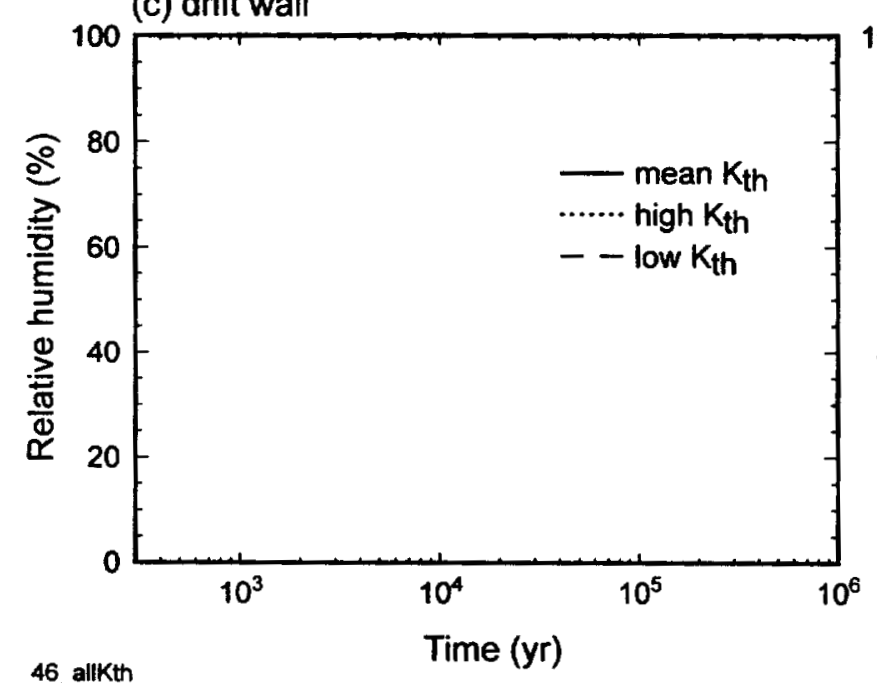

(b) drip shield

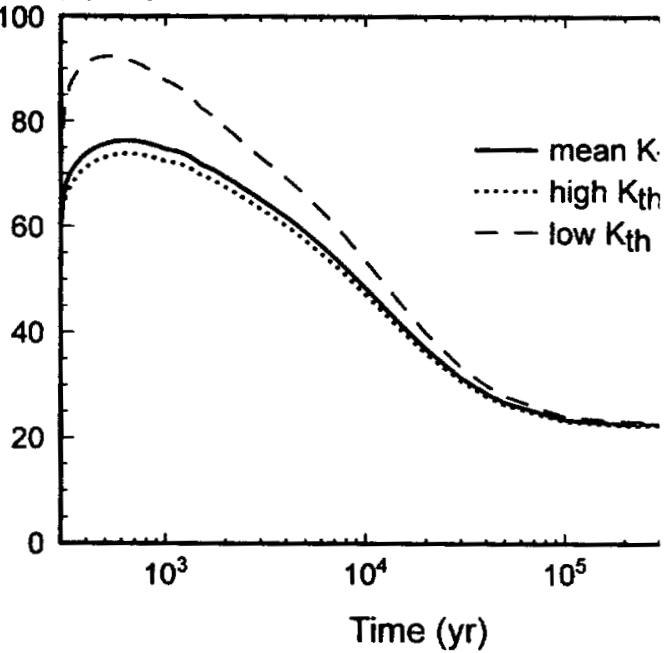

(d) drip shield

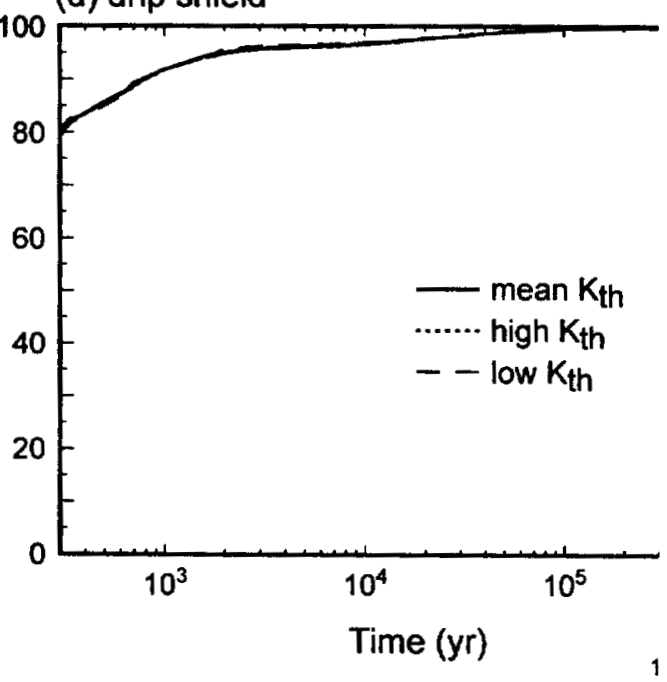

154_0501.ai

Source: Produced using files from Buscheck 2001 [DIRS 155012].

Figure 37 Temperature and relative humidity at the drift wall and drip shield as a function of host-rock thermal conductivity for the central repository $(15 \mathrm{c3}$, see Figure 22$)$ location of LTOM and the mean infiltration-flux scenario. 


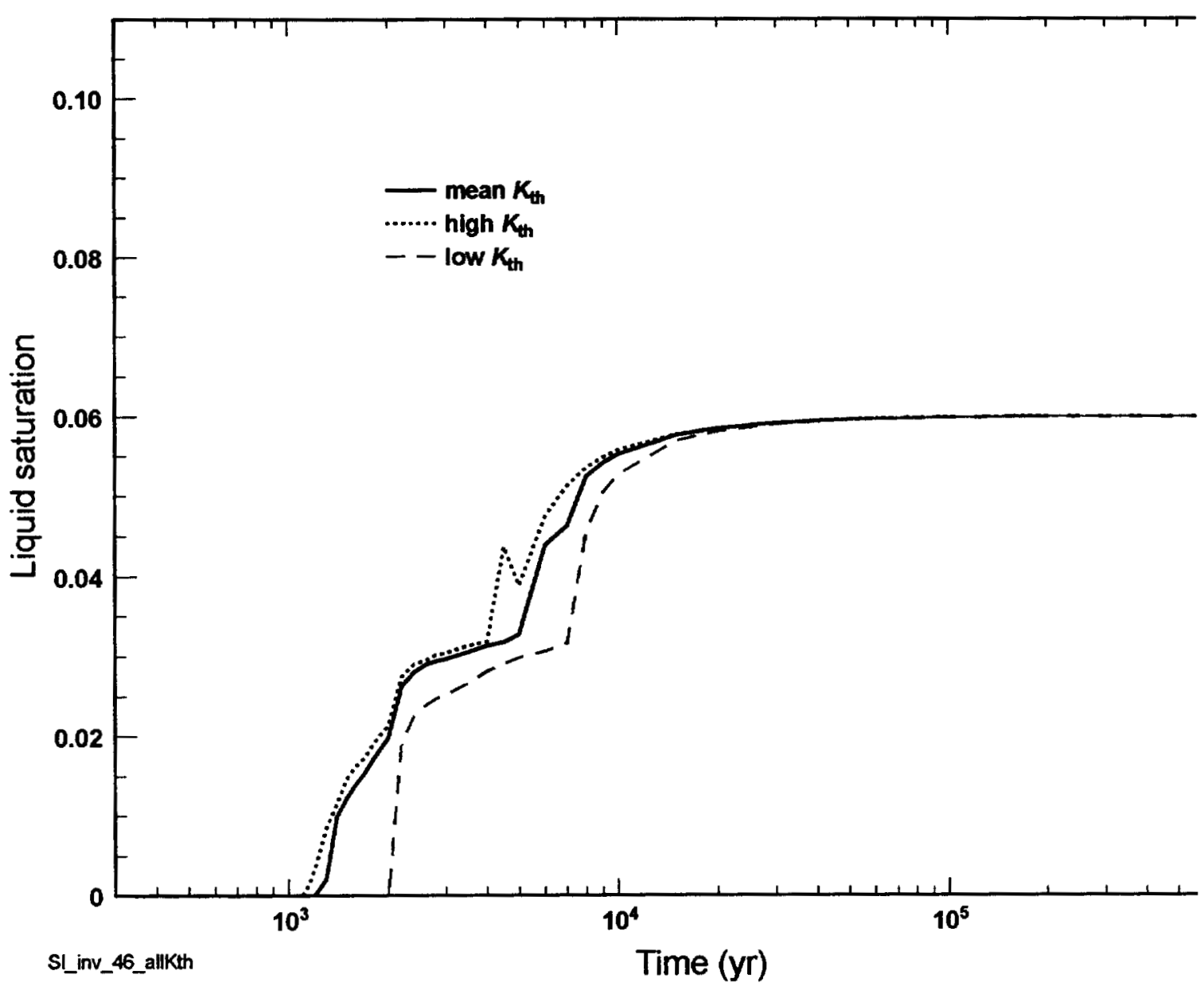

154_0368.ai

Source: Produced using files from Buscheck 2001 [DIRS 155012].

Figure 38 Liquid-saturation in the Invert as a Function of Host-Rock Thermal Conductivity for the Central Repository (L5C3) Location of LTOM and the Mean Infiltration-Flux Scenario. 
(a) drift wall

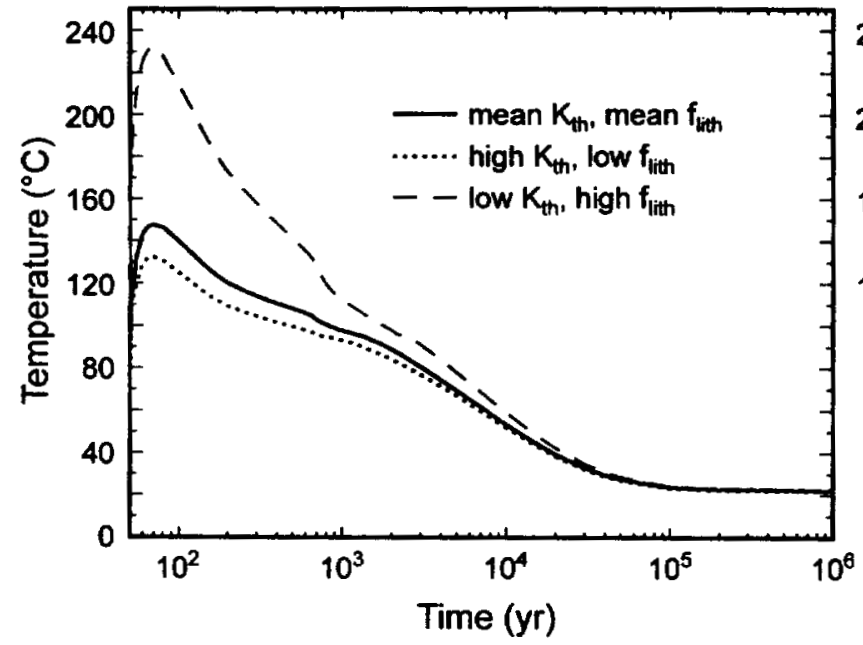

(b) drip shield

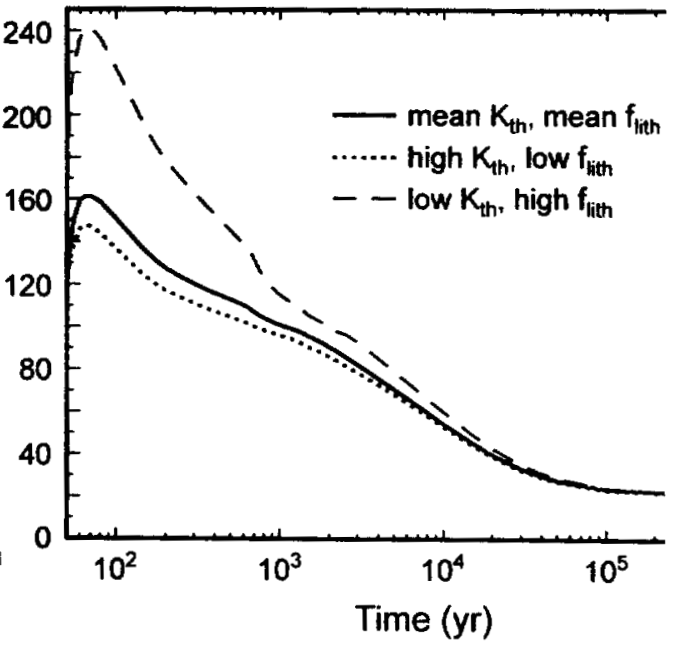

(d) drip shield

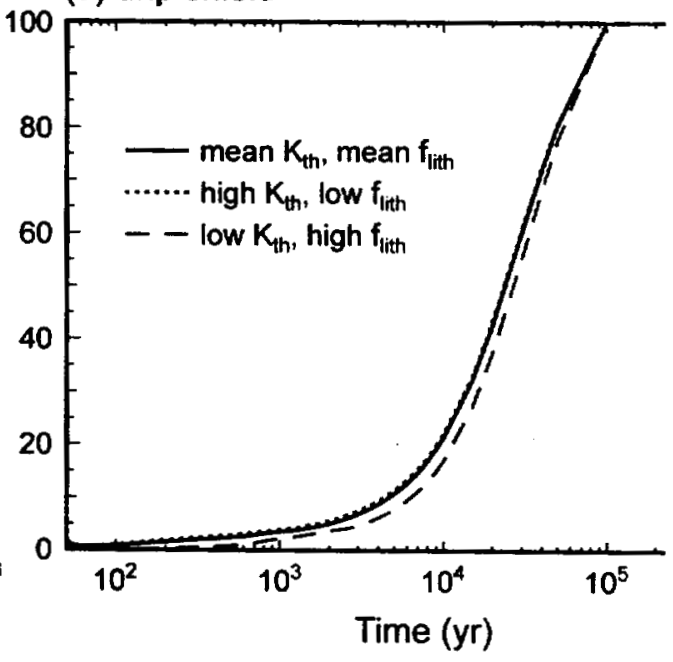

55_allKth_allpor

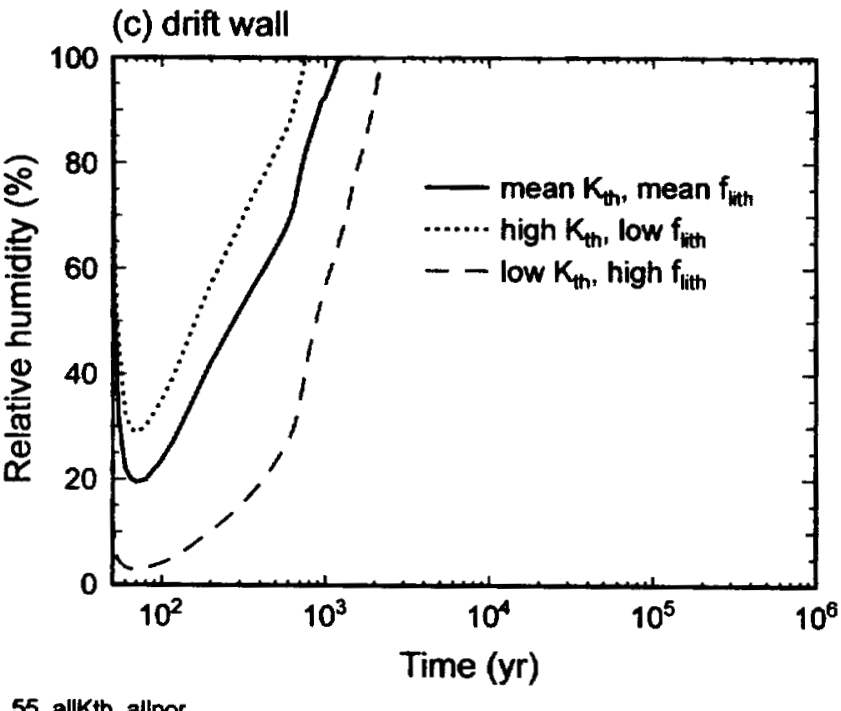

154_0364.ai

Source: Produced using files from Buscheck 2001 [DIRS 155012].

Figure 39 HTOM as a function of mean, high, and low lithophysal porosity ( $\phi_{\text {iith }}$ ) for the mean infiltrationflux scenario. 


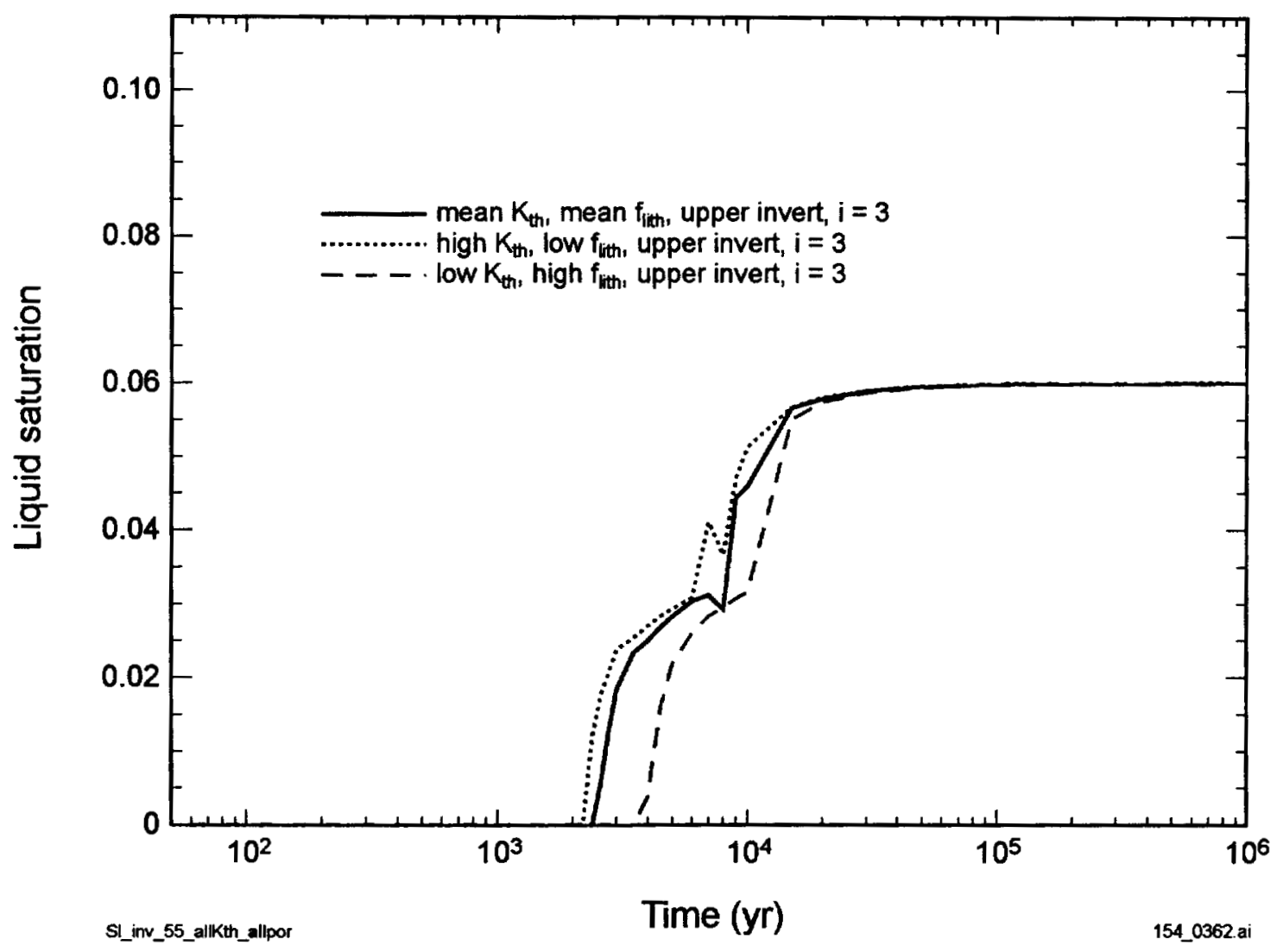

154_0362.ai

Source: Produced using files from Buscheck 2001 [DIRS 155012].

NOTE:Higher-temperature operating mode invert liquid saturations at the central repository location (Location $\mathrm{L} 5 \mathrm{C} 3$ ) as a function of mean, high, and low lithophysal porosity $\left(\mathrm{f}_{\mathrm{lith}}\right)$ associated with appropriate thermal conductivity $\left(\mathrm{K}_{\mathrm{th}}\right)$ for the mean infiltration-flux scenario.

Figure 40 HTOM invert liquid saturations as a function of mean, high, and low lithophysal porosity ( $\left.\phi_{\text {hith }}\right)$ for the mean infiltration-flux scenario. 
(a) drift wall

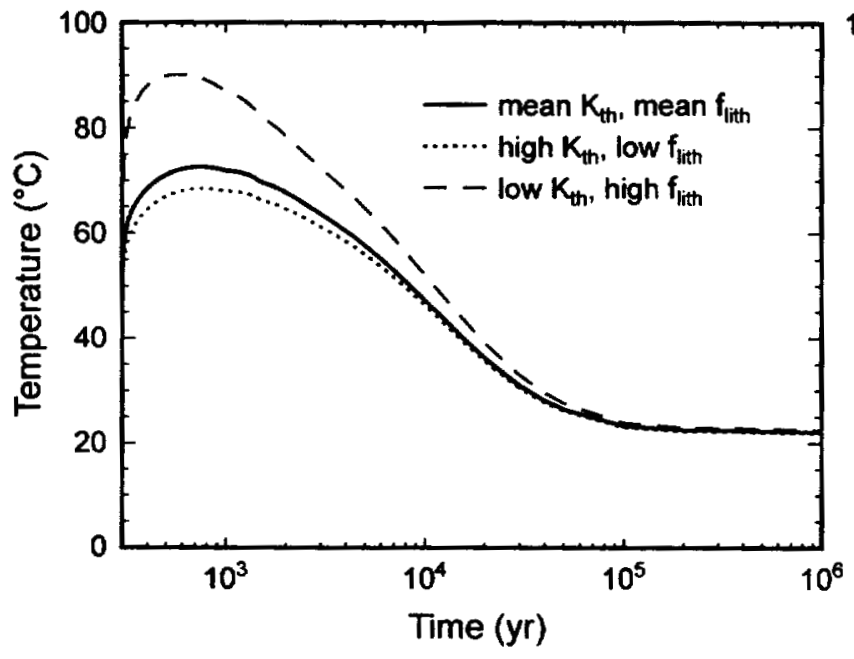

(c) drift wall

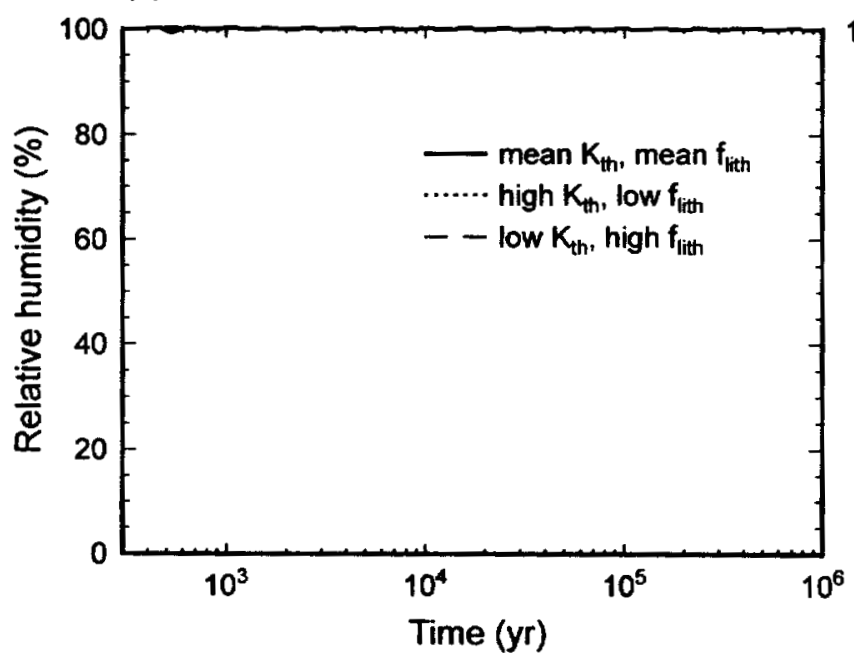

46_allKth_allpor (b) drip shield

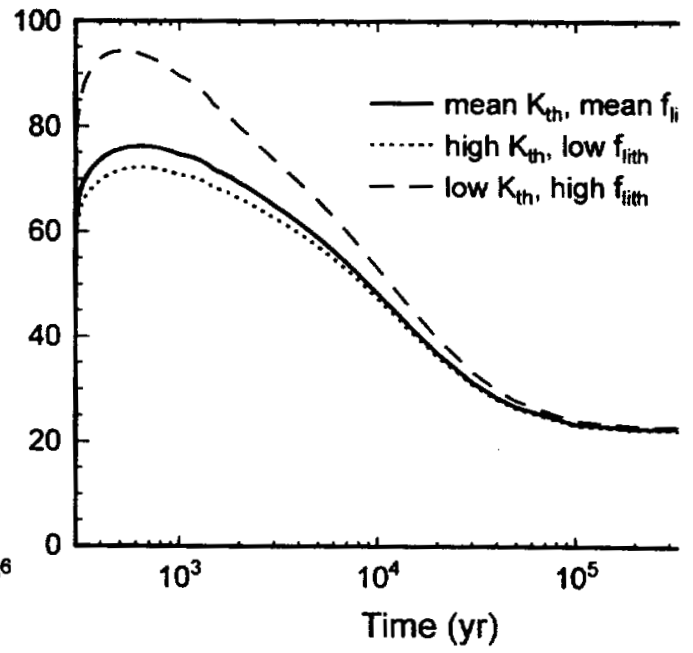

(d) drip shield

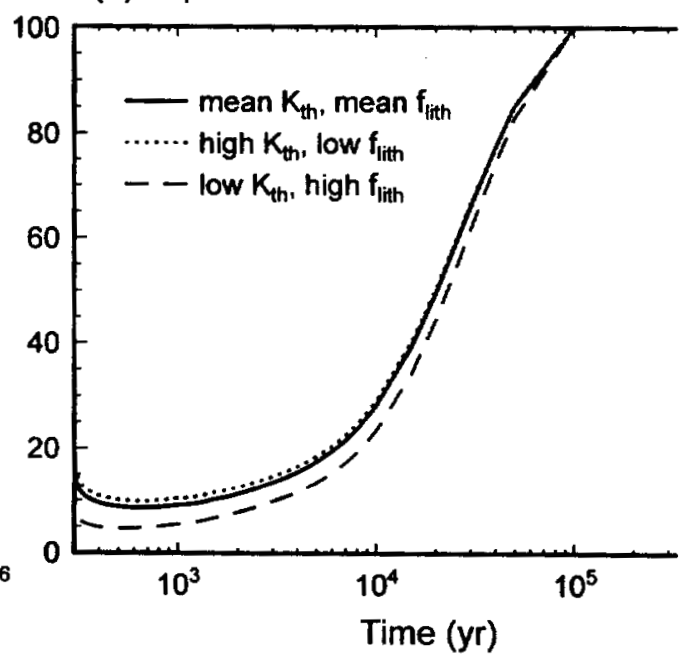

154_0363.ai

Source: Produced using files from Buscheck 2001 [DIRS 155012].

NOTE:Lower-temperature operating mode drift wall and drip shield temperatures and relative humidities at the central repository location (Location L5C3) as a function of mean, high, and low lithophysal porosity $\left(f_{\text {lith }}\right)$ associated with appropriate thermal conductivity $\left(\mathrm{K}_{\mathrm{th}}\right)$ for the mean infiltration-flux scenario.

Figure 41 LTOM as a function of mean, high, and low lithophysal porosity ( $\left.\phi_{\text {tith }}\right)$ for the mean infiltrationflux scenario. 


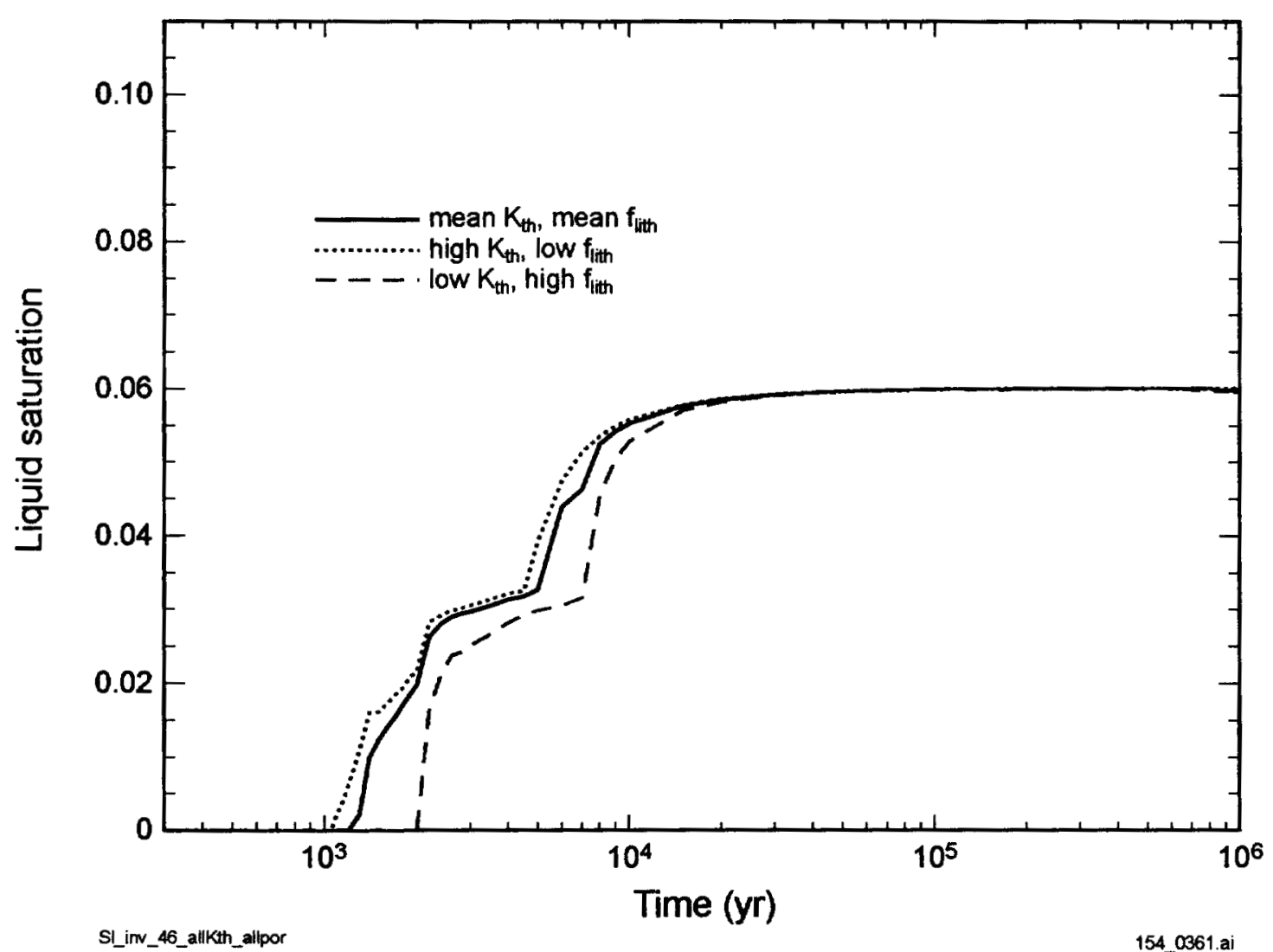

154_0361.ai

Source: Produced using files from Buscheck 2001 [DIRS 155012].

Figure 42 LTOM invert saturation for the central repository location (15c3) as a function of mean, high, and low lithophysal porosity ( $\phi_{\text {ith }}$ ) associated with appropriate thermal conductivity $\left(k_{\text {lith }}\right)$ for the mean infiltration-flux scenario. 


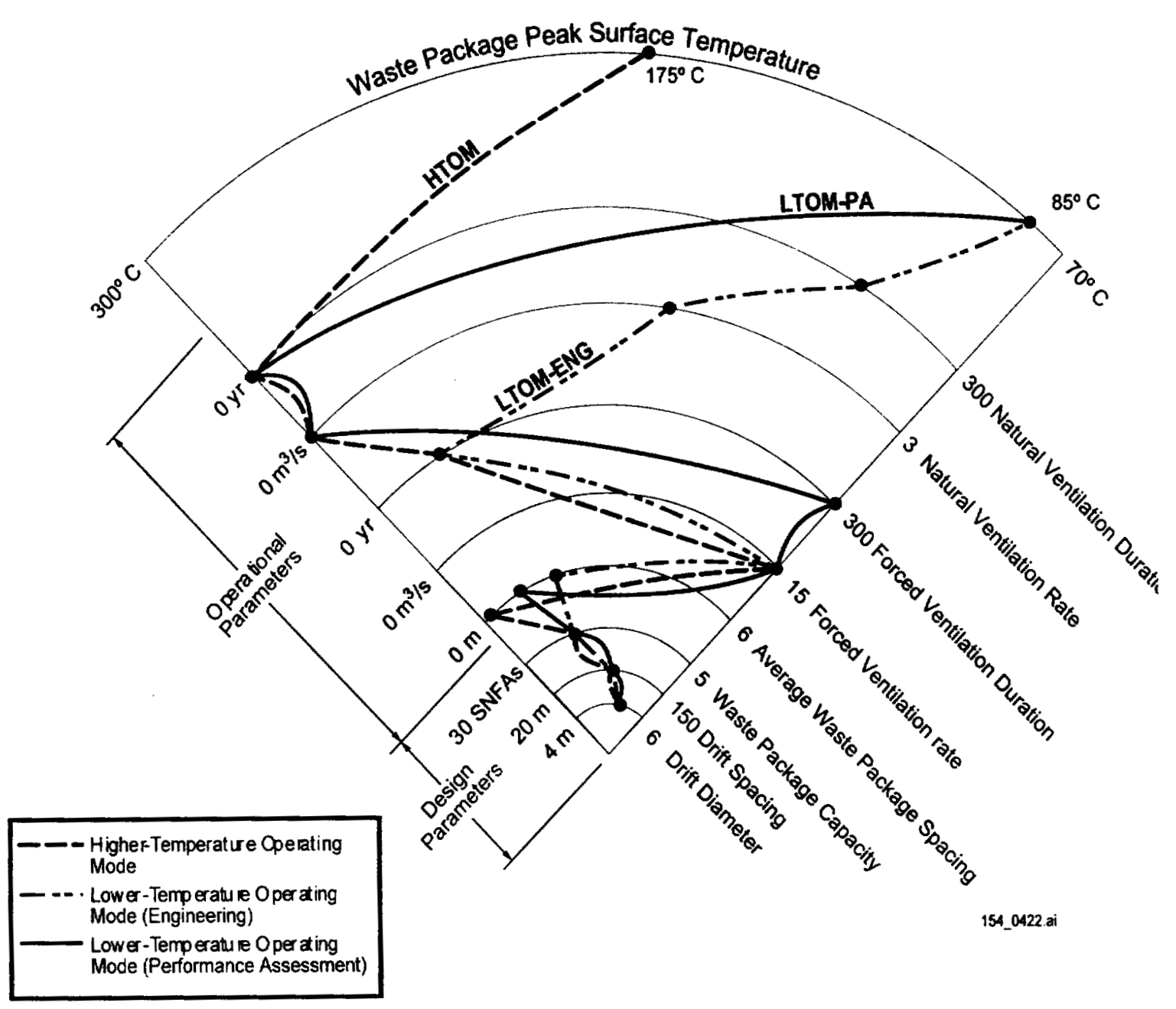

154_0422.ai

Source: Produced from information in BSC 2001 [DIRS 154864] and BSC 2001 [DIRS 155010].

NOTE:Two sets of parameters are shown that can meet the lower-temperature operating mode goal. Black dots represent values of design and operating parameters which, when combined, result in the peak waste package temperatures shown. Lines through the black dots represent three combinations of parameters being evaluated during the SR time period. HTOM = higher-temperature operating mode; $P A=$ performance assessment base case; $\mathrm{ENG}=$ engineering evaluations.

Figure 43 Design and Operating Mode Parameters Used to Meet Peak Waste Package Temperature Goals. 
(a) Peak Waste-Package Temperature

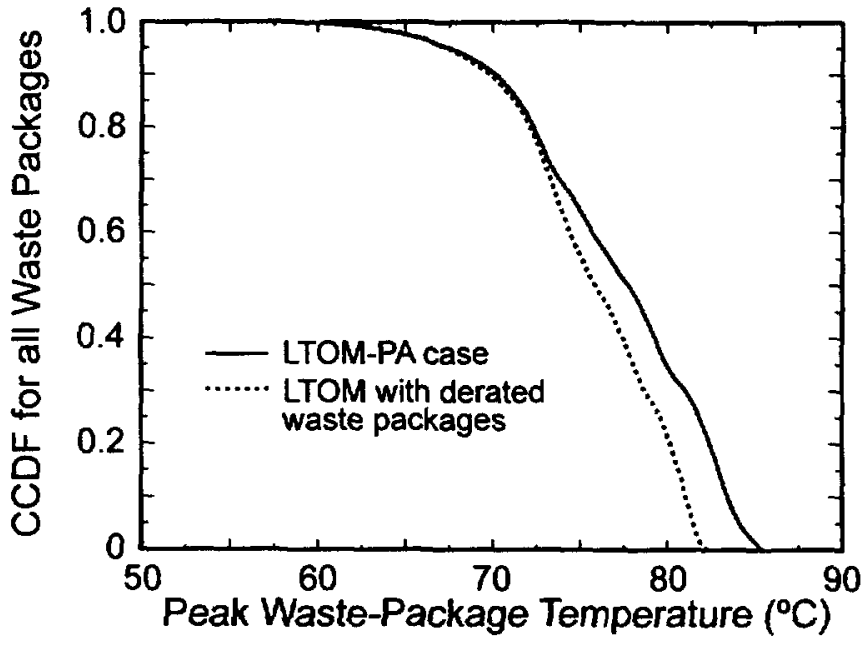

(b) Waste-Package Relative Humidity when $T_{\text {wp }}=85^{\circ} \mathrm{C}$

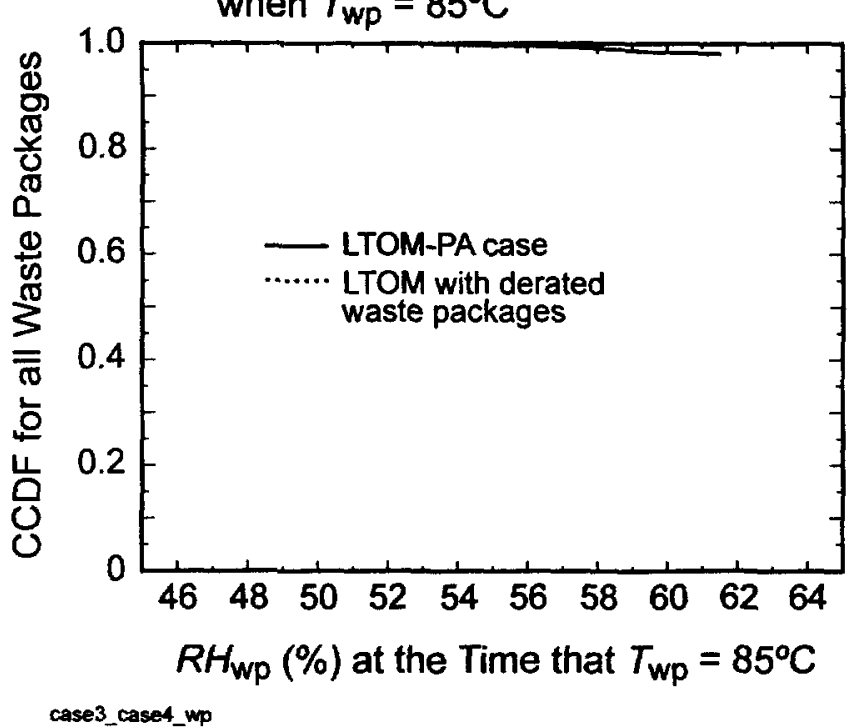

154_0423a.ai

154_0423a.ai

Source: Produced using files from Buscheck 2001 [DIRS 155449].

NOTE:Temperature and relative humidity $(R H)$ when the waste package temperature $\left(T_{w p}\right)$ is $85^{\circ} \mathrm{C}$ considering repository footprint, infiltration flux map, and variability among waste packages. LTOM-PA = lower-temperature operating mode used in the performance assessment base case. $\mathrm{CCDF}=$ complementary cumulative distribution function.

Figure 44 Waste package temperature and relative humidity for two methods of achieving LTOM conditions. 


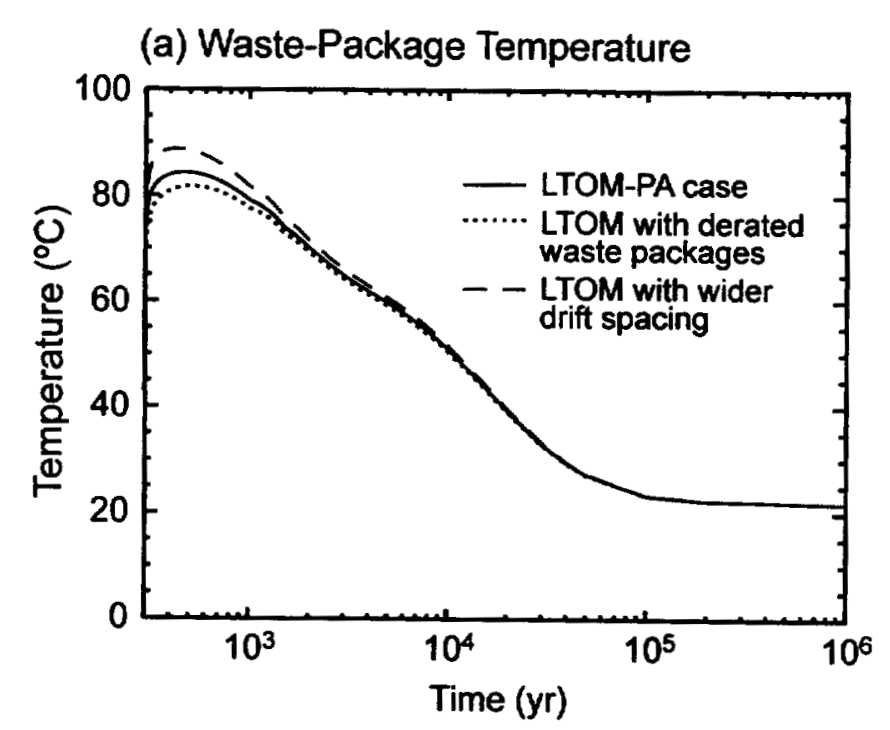

(b) Waste-Package Relative Humidity

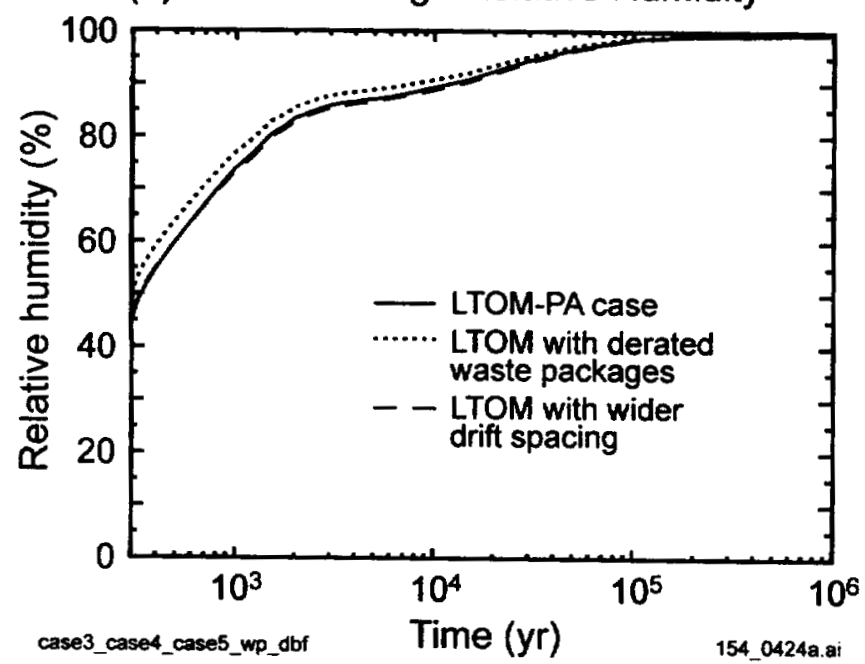

154_0424a.ai

Source: Produced using files from Buscheck 2001 [DIRS 155449].

NOTE:Figure depicts three lower-temperature operating mode cases using the multiscale thermal-hydrologic model at the L5C3 location in the footprint of the potential repository. LTOM = lower-temperature operating mode.

Figure 45 Temperature and relative humidity histories at the waste package surface for three LTOM designs. 


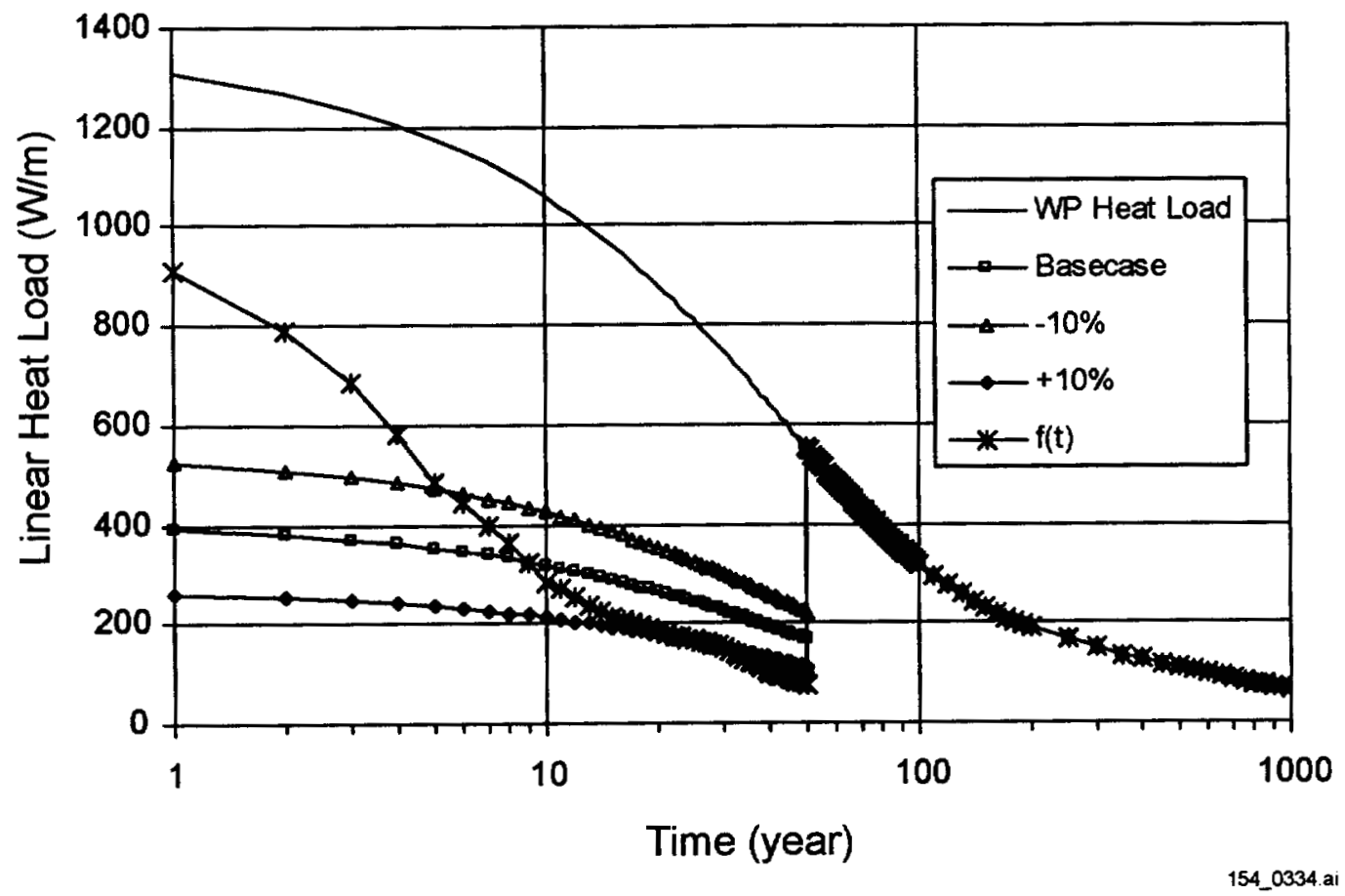

154 0334.ai

Source: Produced using files from Leem 2001 [DIRS 155457].

NOTE: $W P=$ waste package.

Figure 46 Time History of Heat Available to Enter the Near-Field Rock for the HTOM Ventilation Cases. 


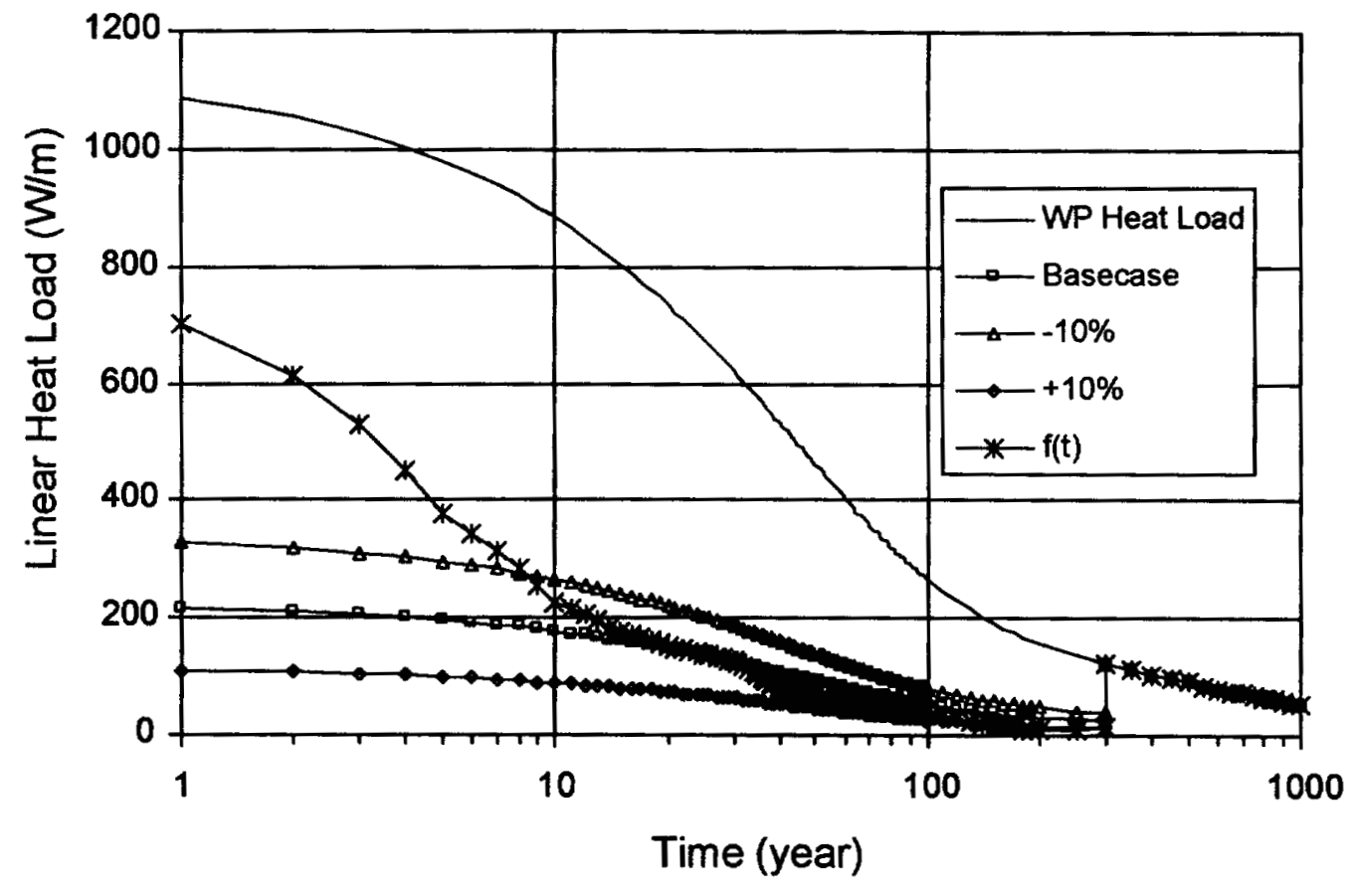

154_0340.ai

Source: Produced using files from Leem 2001 [DIRS 155457].

Figure 47 Time History of Heat Available to Enter the Near-Field Rock for the LTOM Ventilation Cases. 
(a) HTOM

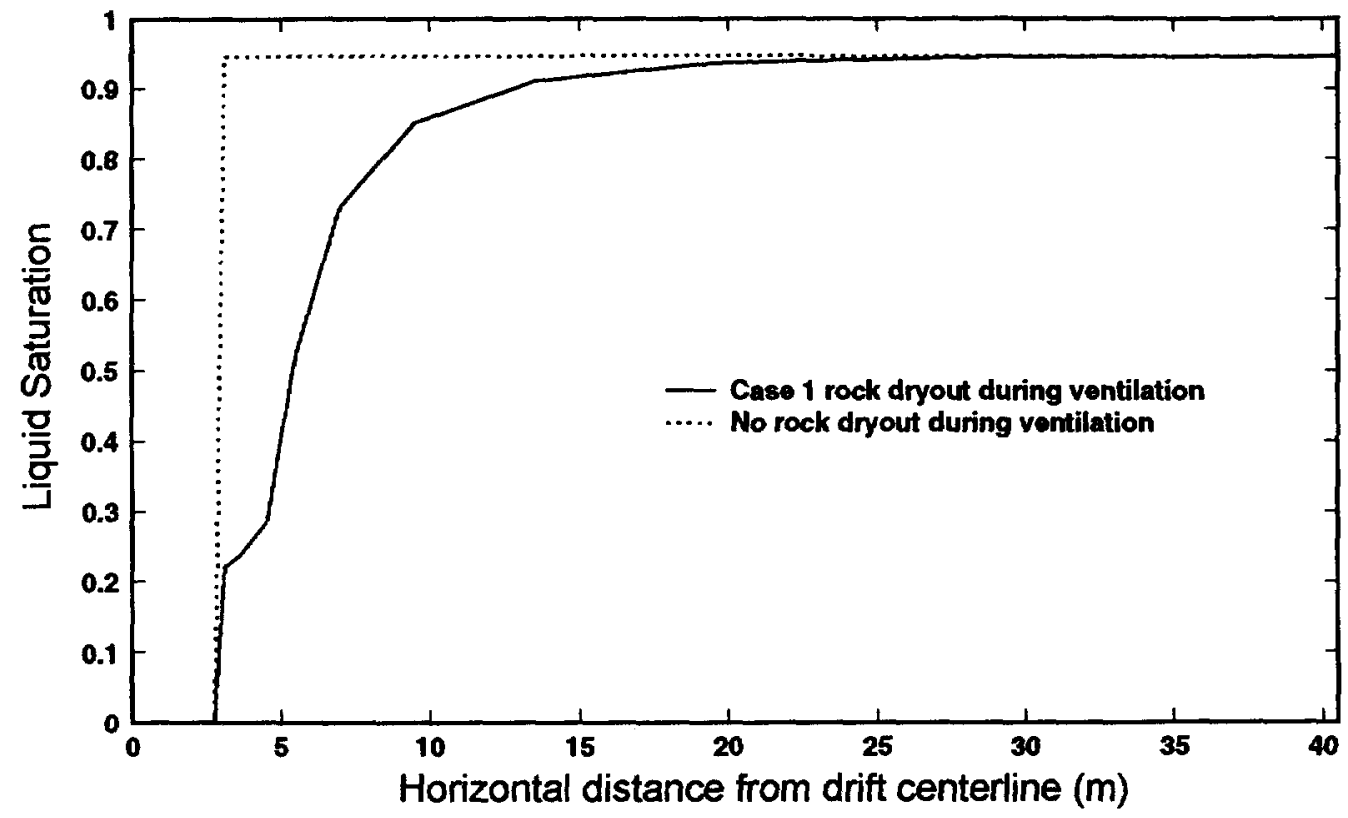

(b) LTOM

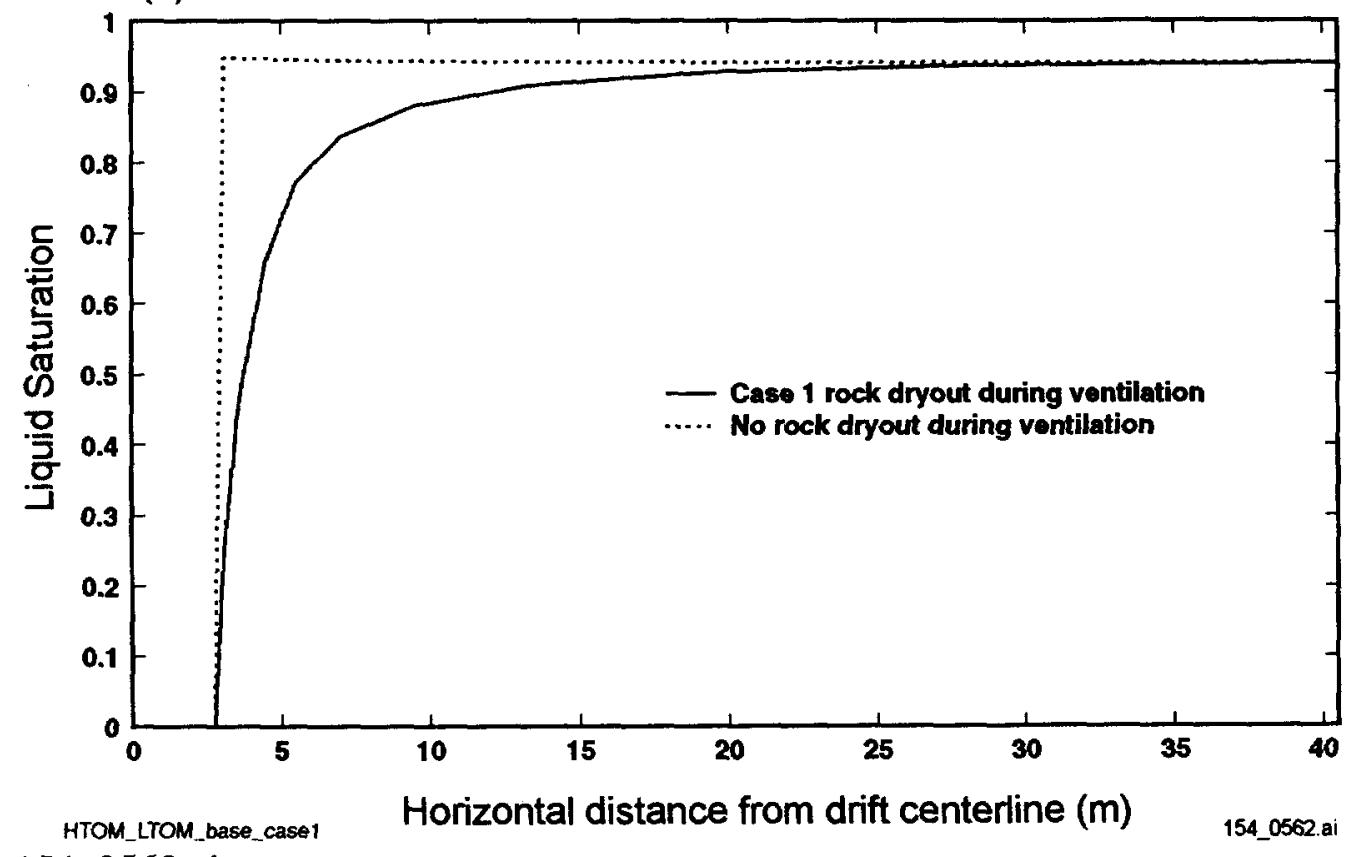

154_0562.ai

Source: Buscheck 2001 [DIRS 155012].

NOTE: The liquid saturations pertain to the matrix continuum.

Figure 48 Lateral Extent of Host-Rock Dryout Due to Ventilation for Case 1 for the HTOM and LTOM. 
(a) Drift Wall

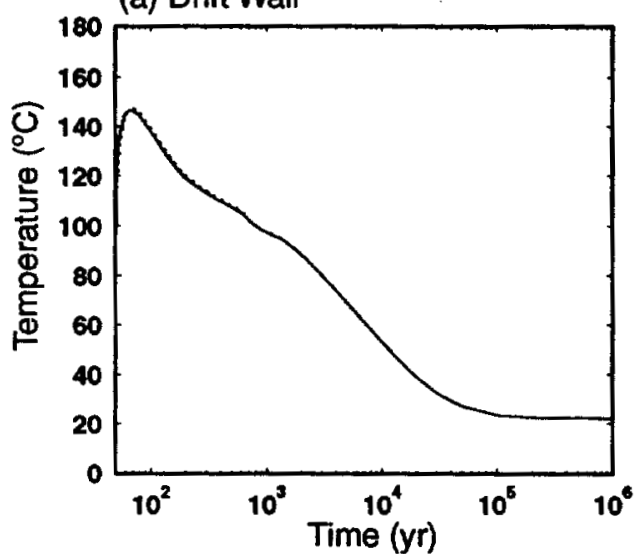

(c) Drift Wall

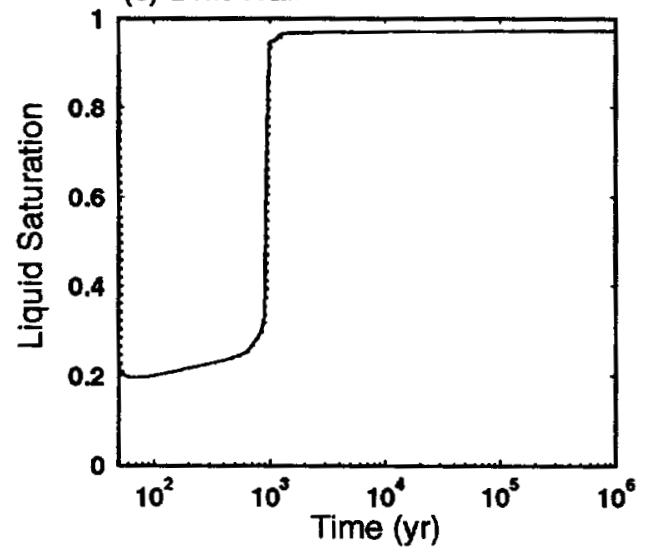

(e) Invert

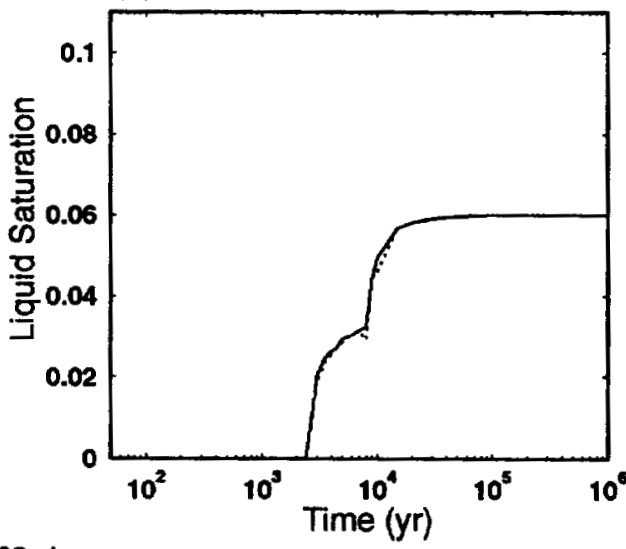

(b) Drip Shield

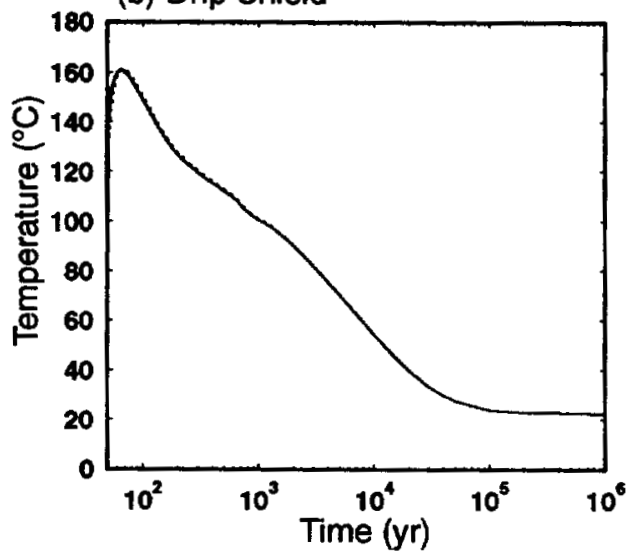

(d) Drip Shield

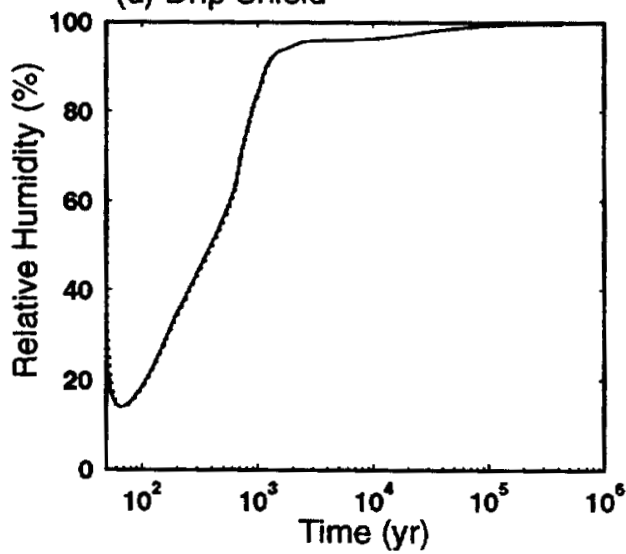

LTOM Legend

- Case 1 rock dryout during ventllation No rock dryout during ventilation

HTOM_base_case 1

154_0563.ai

154_0563.ai

Source: Buscheck 2001 [DIRS 155012].

NOTE:The results for the base-case LDTH submodel that neglects the influence of preclosure dryout are also given. The drift wall liquid saturation pertains to the matrix continuum.

Figure 49 Temperature of the drift wall and the drip shield, relative humidity on the drip shield, and liquid saturation at the drift wall and in the invert for Case 1 of the HTOM. 
(a) Drift Wall

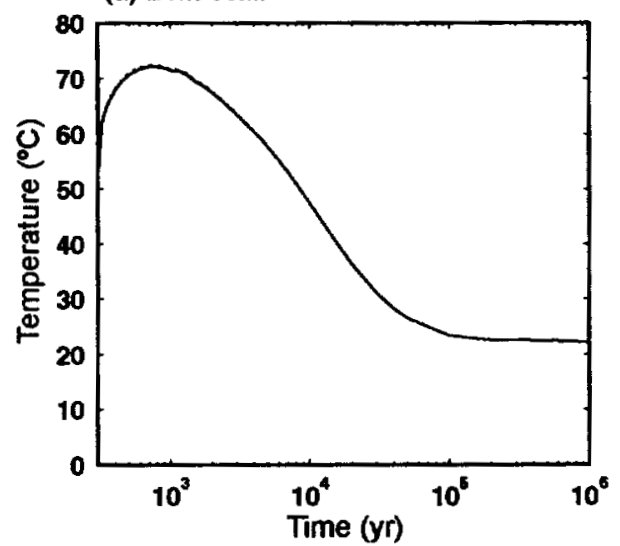

(c) Drift Wall

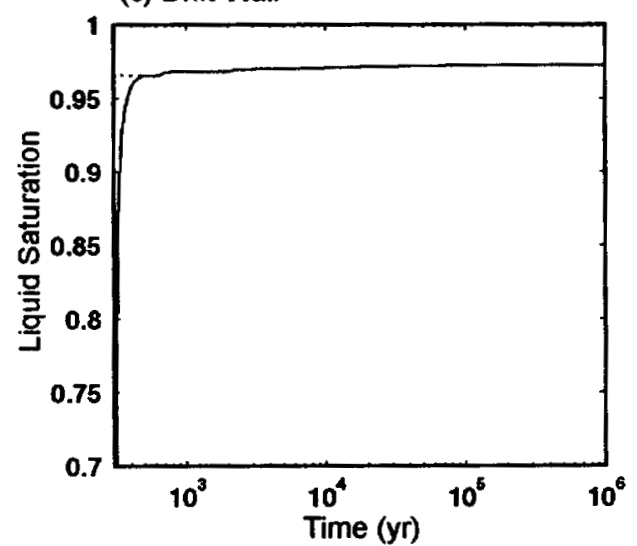

(e) Invert

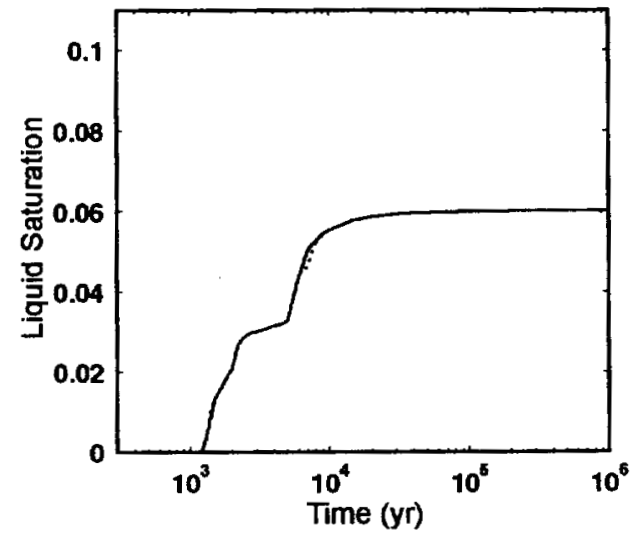

(b) Drip Shield

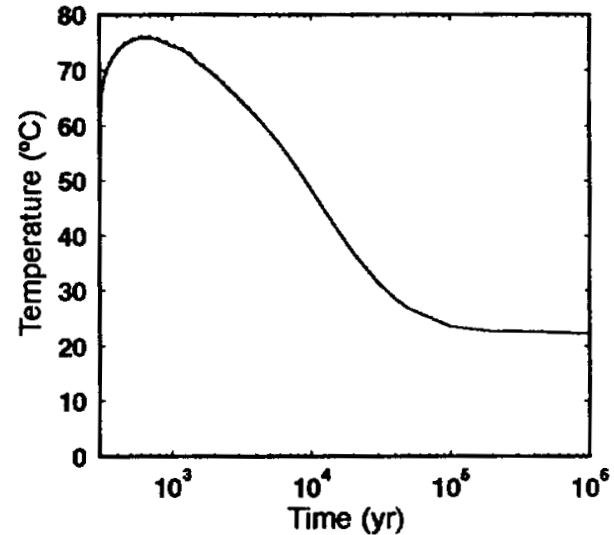

(d) Drip Shield

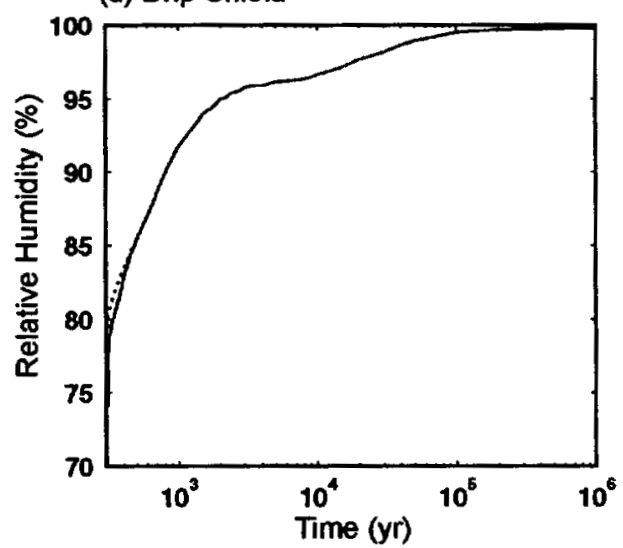

LTOM Legend

- Case 1 rock dryout during ventilation ..... No rock dryout during ventilation

tTOM_base_caso1

154. 0564.8i

154_0564.ai

Source: Buscheck 2001 [DIRS 155012].

NOTE:The results for the base-case LDTH submodel that neglects the influence of preclosure dryout are also given. The drift wall liquid saturation pertains to the matrix continuum.

Figure 50 Temperature of the Drift Wall and the Drip Shield, Relative Humidity on the Drip Shield, and Liquid Saturation at the Drift Wall and in the Invert for Case 1 of the LTOM. 


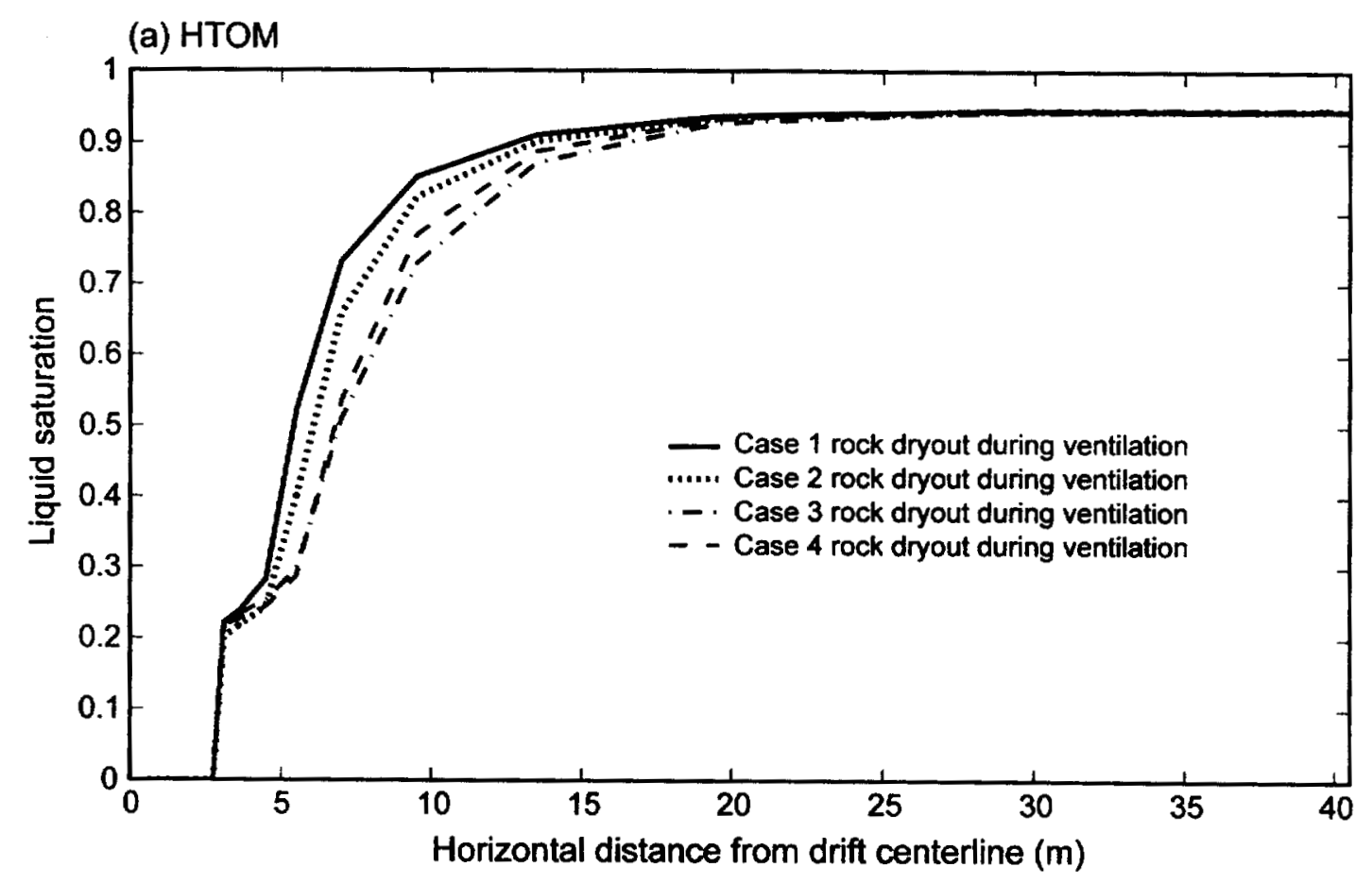

(b) LTOM

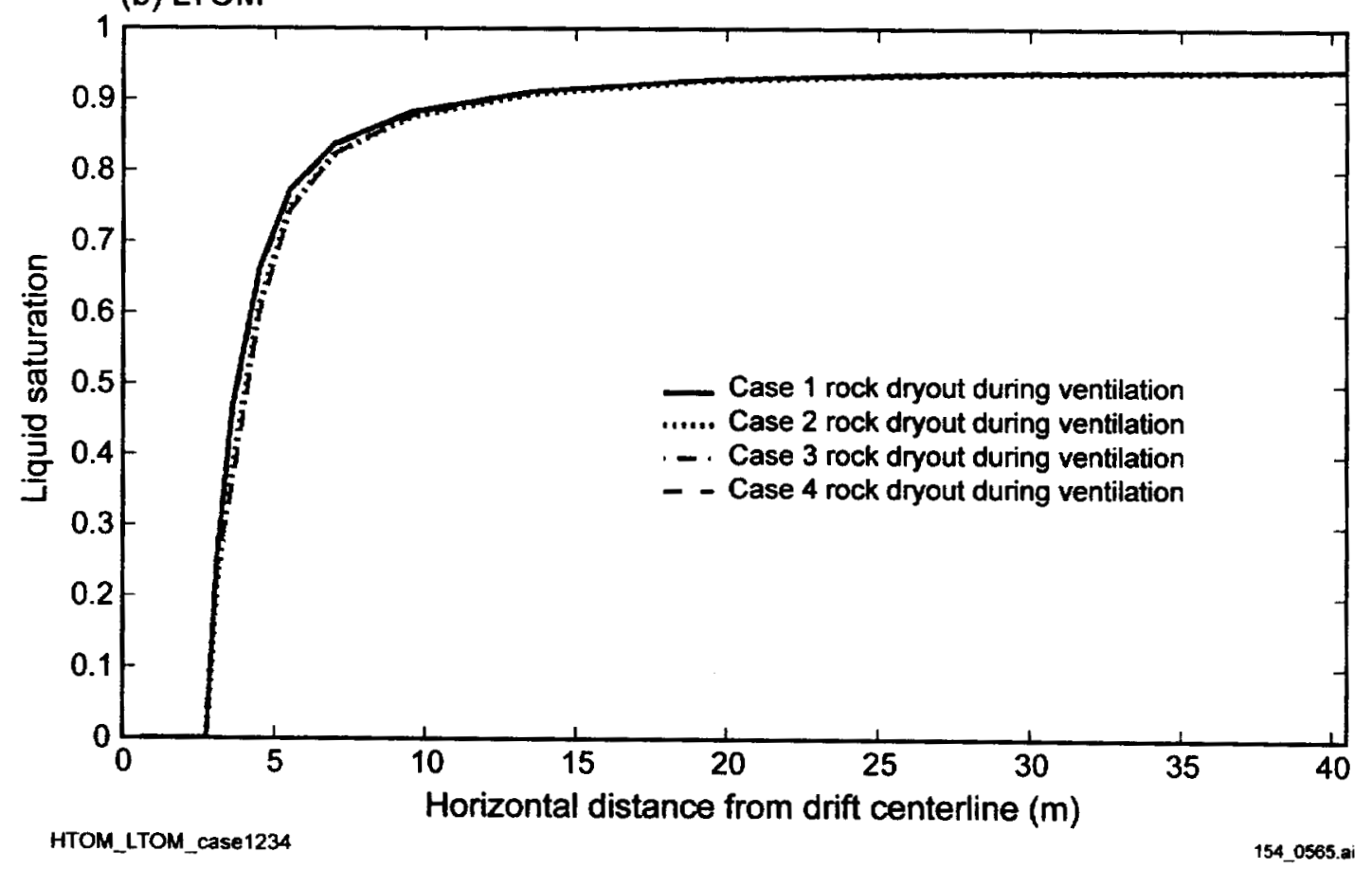

154_0565.ai

Source: Buscheck 2001 [DiRS 155012].

NOTE: The liquid saturations pertain to the matrix continuum.

Figure 51 Lateral Extent of Host-rock Dryout Due to Ventilation for Cases 1, 2, 3, and for HTOM and LTOM. 
(a) Drift Wall

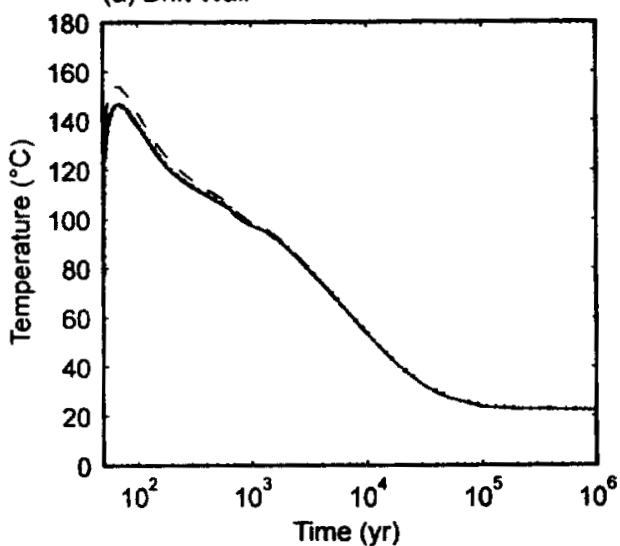

(c) Drift Wall

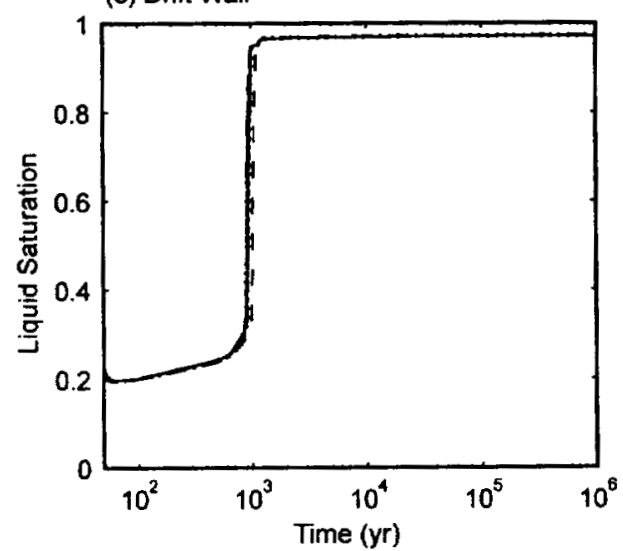

(e) Invert

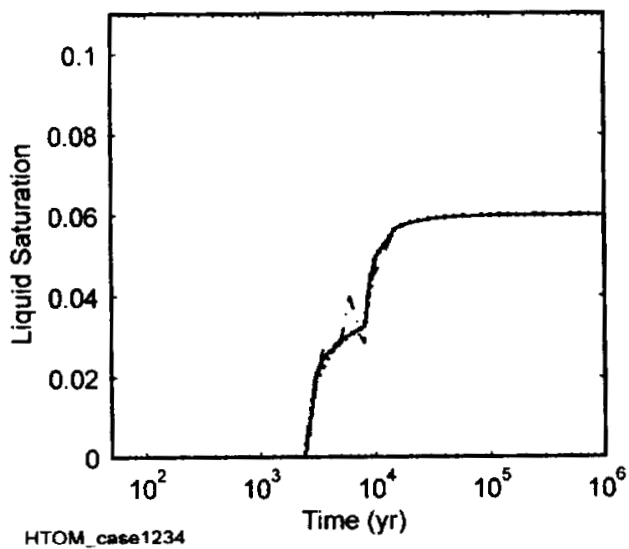

(b) Drip Shield

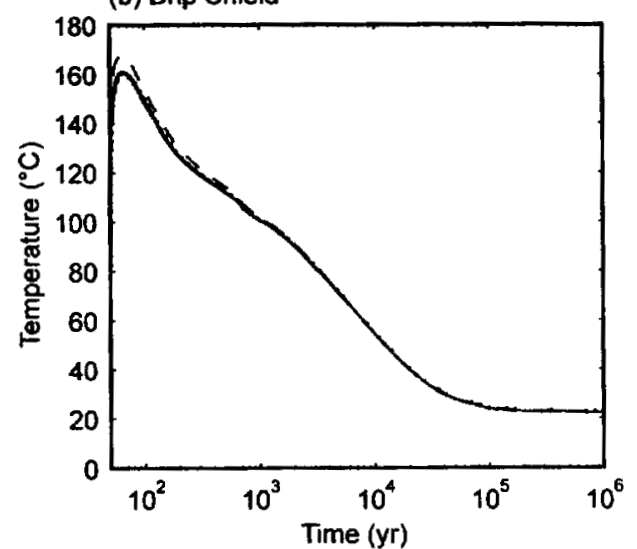

(d) Drip Shield

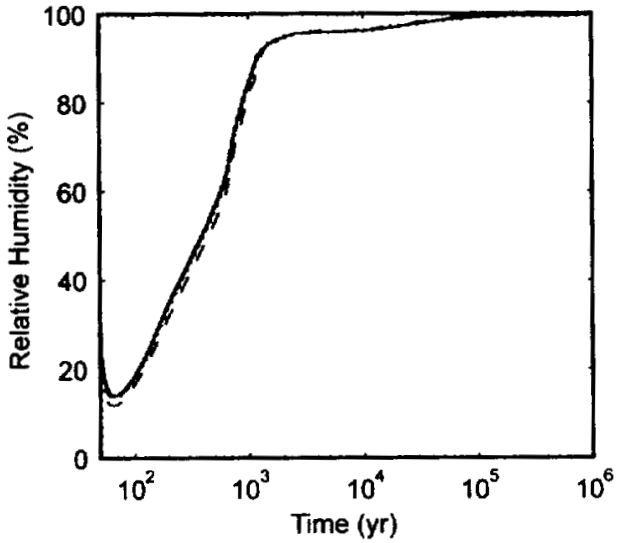

HTOM Legend

- Case $\uparrow$ rock dryout during ventilation

..... Case 2 rock dryout during ventilation

- Case 3 rock dryout during ventilation

- Case 4 rock dryout during ventilation

154 0566.ai

Source: Buscheck 2001 [DIRS 155012].

NOTE: The drift wall liquid saturation pertains to the matrix continuum.

Figure 52 Temperature of the Drift Wall and the Drip Shield, Relative Humidity on the Drip shield, and Liquid Saturation at the Drift Wall and in the Invert for Cases 1, 2, 3, and 4 of HTOM. 
(a) Drift Wall

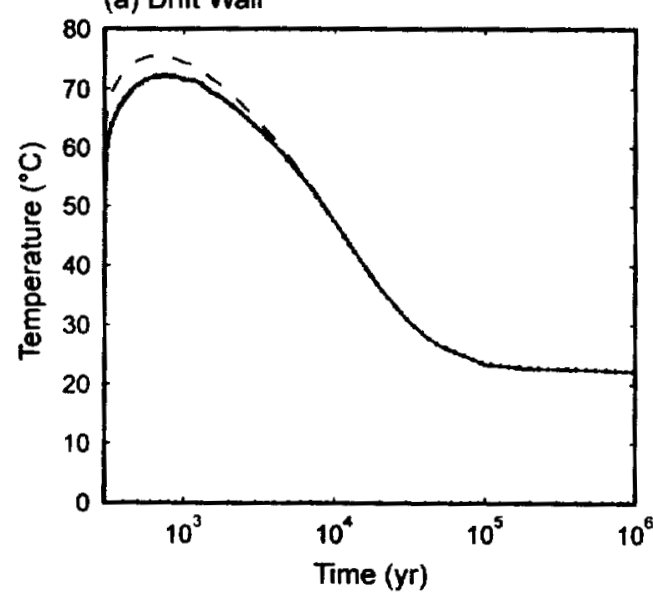

(c) Drift Wall

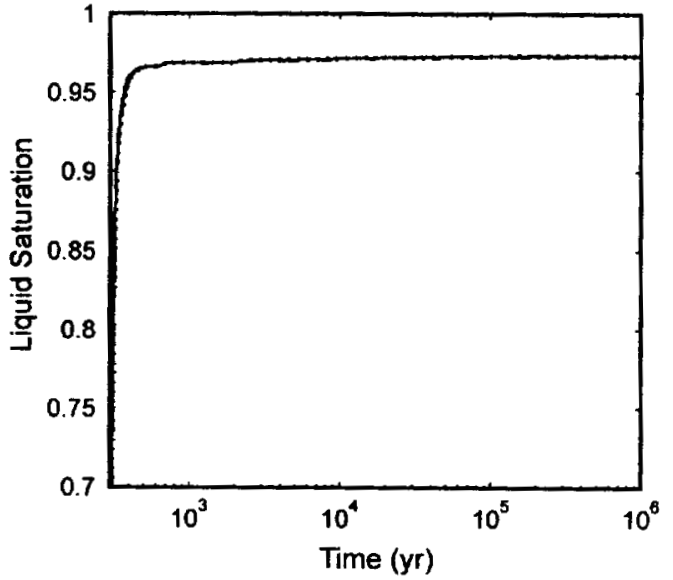

(e) Invert

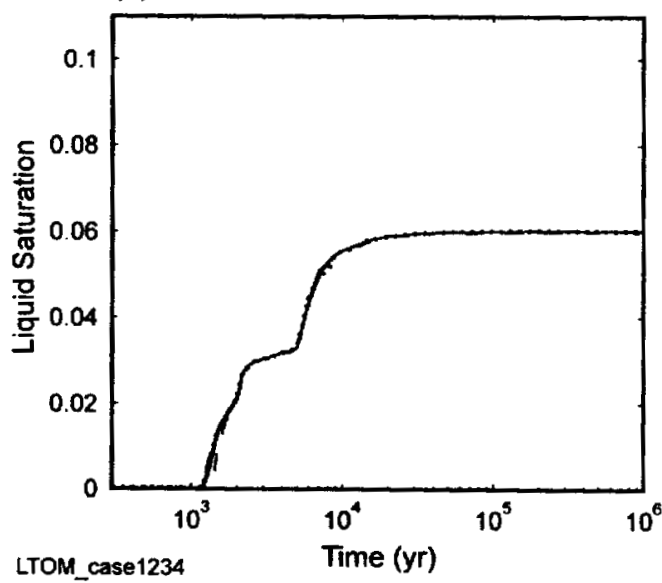

(b) Drip Shield

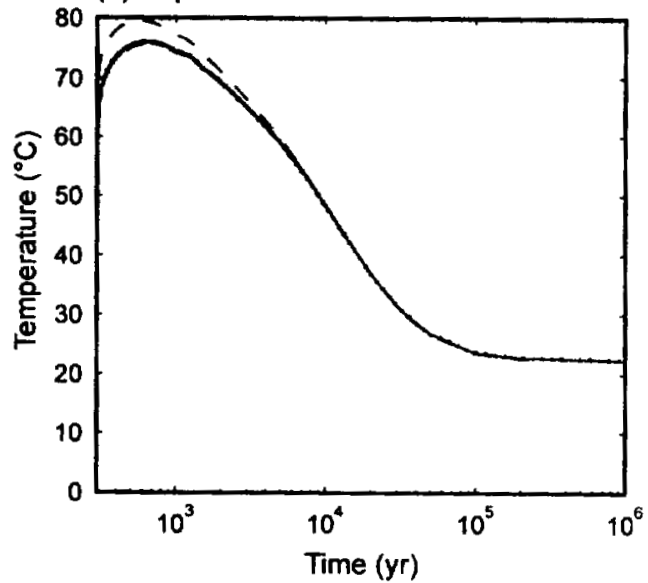

(d) Drip Shield

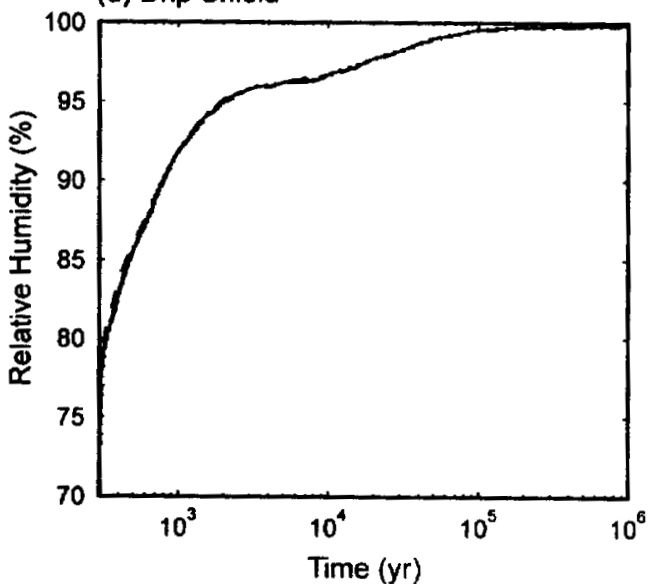

LTOM Legend

- Case 1 rock dryout during ventilation

...... Case 2 rock dryout during ventilation

- Case 3 rock dryout during ventilation

- Case 4 rock dryout during ventilation

154 0567.ai

Source: Buscheck 2001 [DIRS 155012].

NOTE: The drift wall liquid saturation pertains to the matrix continuum.

Figure 53 Temperature of the Drift Wall and the Drip Shield, Relative Humidity on the Drip Shield, and Liquid Saturation at the Drift Wall and in the Invert for Cases 1, 2, 3, and 4 of LTOM. 


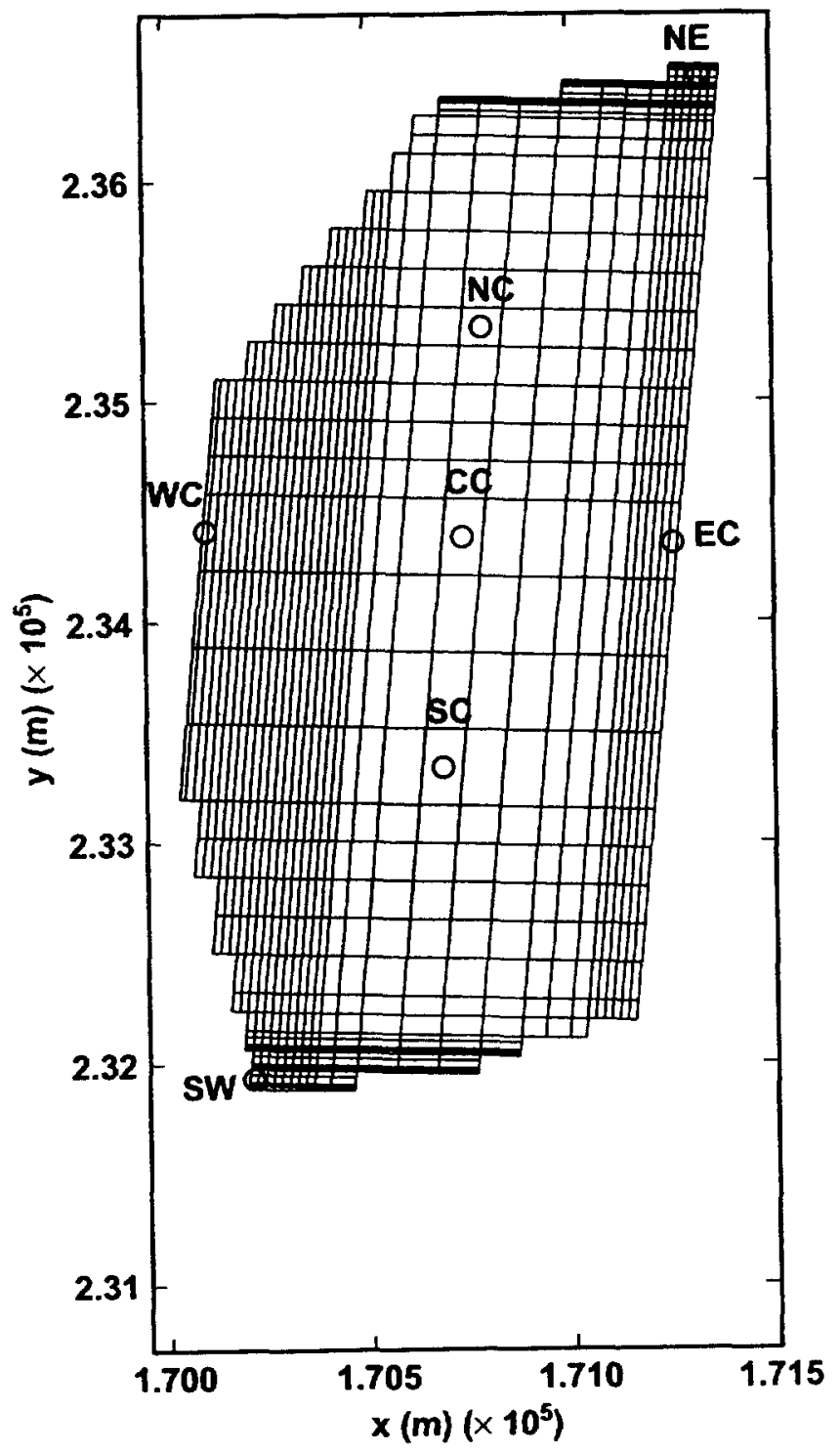

hisCase1Nodes

Source: Produced using files from Buscheck 2001 [DIRS 155449].

NOTE:Plan view of the potential Higher-Temperature Operating Mode repository showing the geographic locations considered in various analyses. $\mathrm{X}=$ east-west distances, $\mathrm{Y}=$ north-south distances.

Figure 54 Location of the seven sites used in analysis of HTOM repository. 
(a) Drift Wall

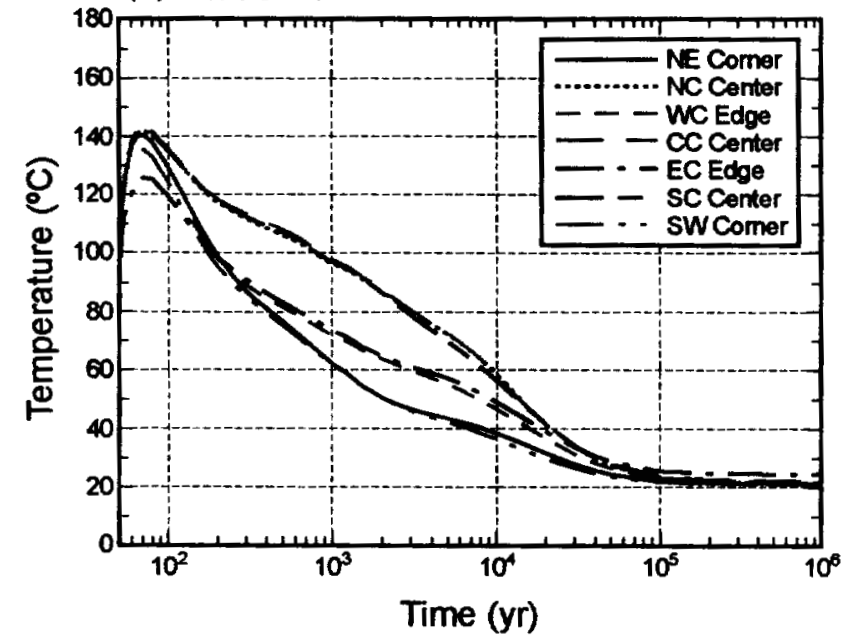

(c) Drift Wall

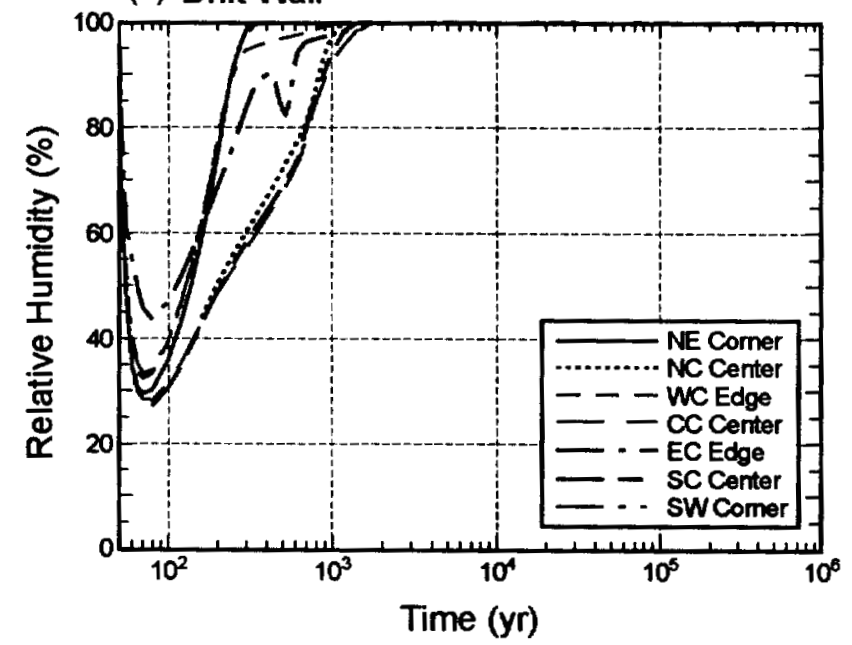

(b) Waste Package

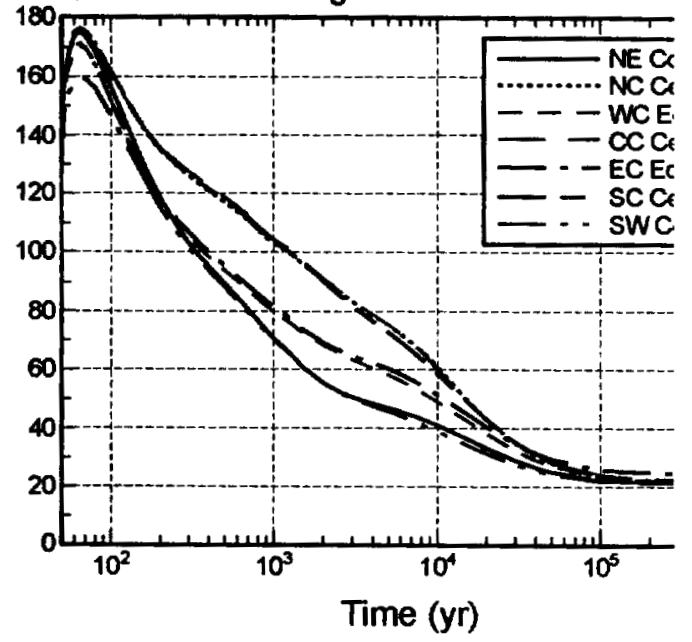

(d) Waste Package

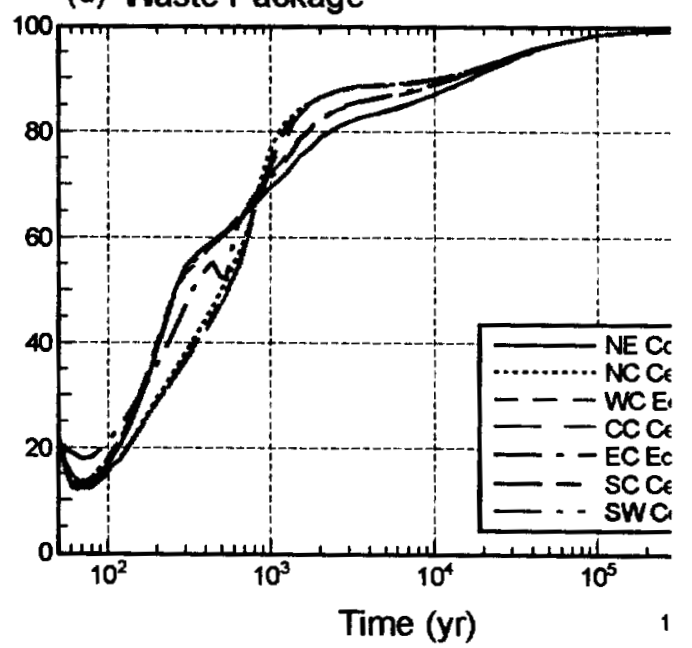

154_0409.ai

Source: Produced using files from Buscheck 2001 [DIRS 155449].

NOTE: Average 21-PWR commercial SNF waste package mean-infiltration flux scenario.

Figure 55 Temperature and relative humidity histories on the drift wall and the waste package for the seven locations in the HTOM repository. 
(a) Drift Wall

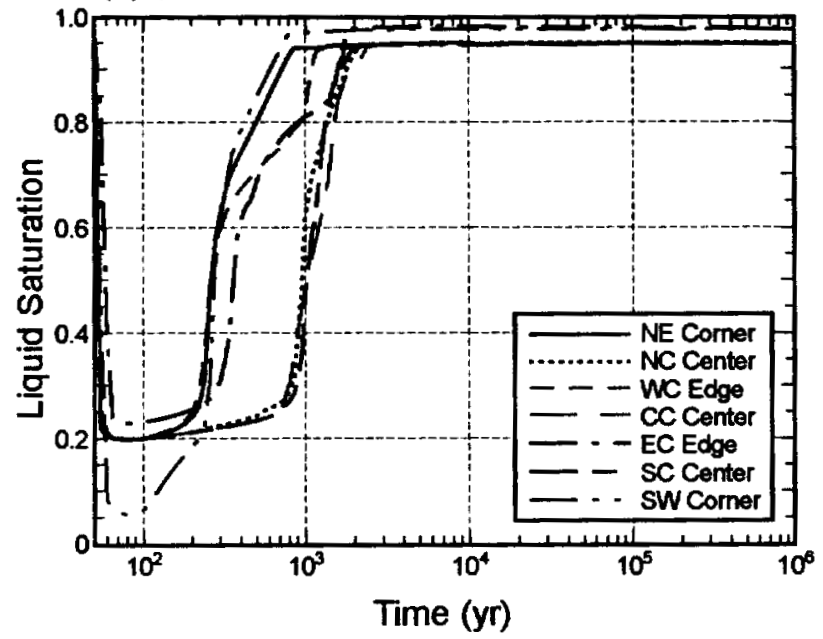

(b) Invert

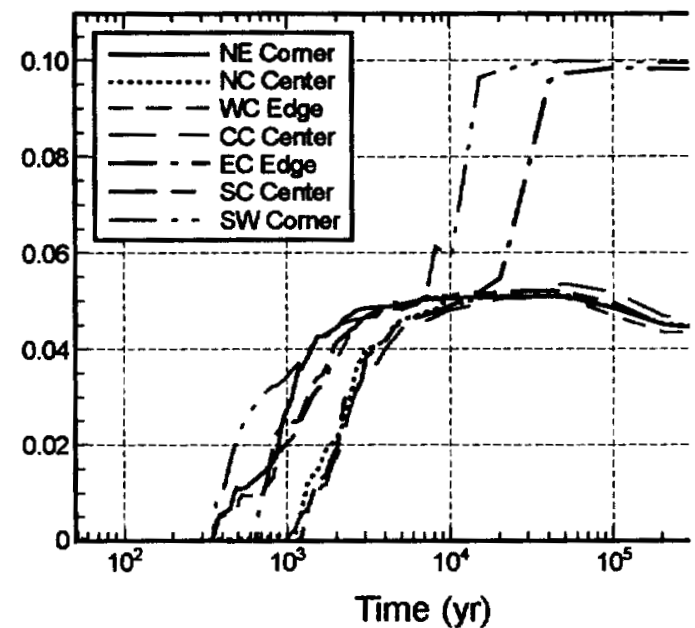

(c) Invert

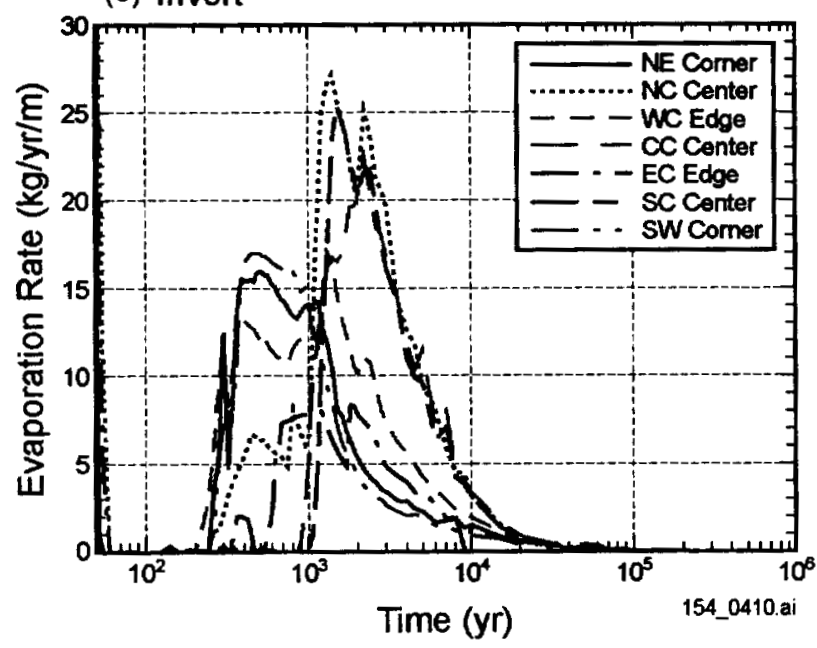

154_0410.ai

Source: Produced using files from Buscheck 2001 [DIRS 155449].

NOTE:Average 21-PWR CSNF waste package Mean-infiltration-flux scenario. Liquidsaturation curves are given for (a) the matrix at the drift wall and for (b) the invert. Evaporation-rate histories are given for the invert (c).

Figure 56 Liquid saturation and evaporation rate histories for the seven locations in the HTOM repository. 
(a) $T_{w p}$ at $50 \mathrm{yr}$

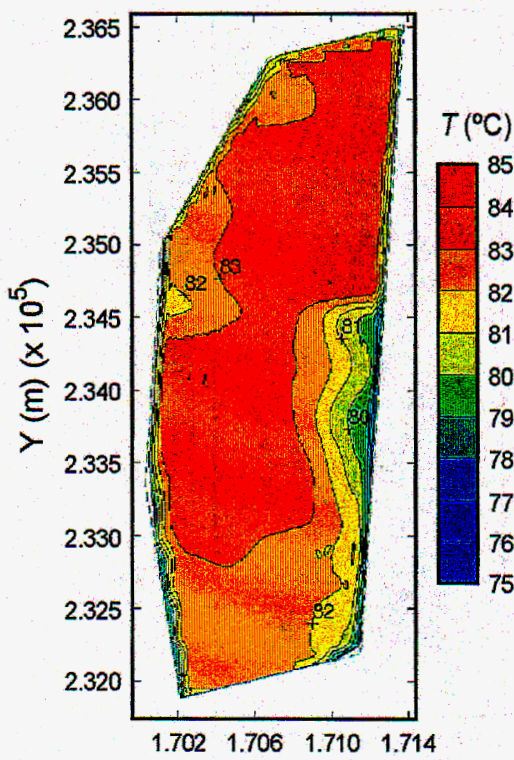

(d) $T_{w p}$ at $2000 \mathrm{yr}$

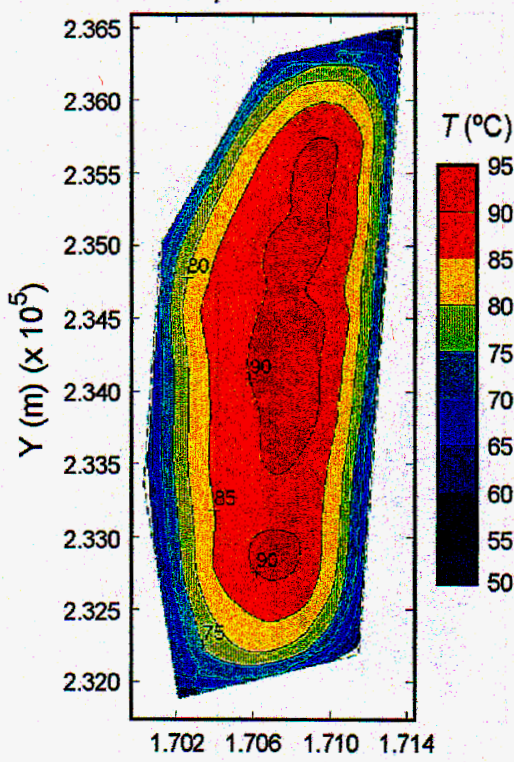

(b) $T_{w p}$ at $60 \mathrm{yr}$

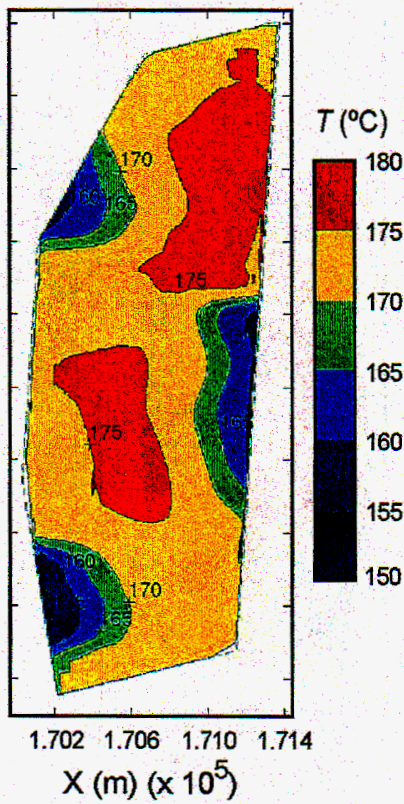

(e) $T_{w p}$ at $10,000 \mathrm{yr}$

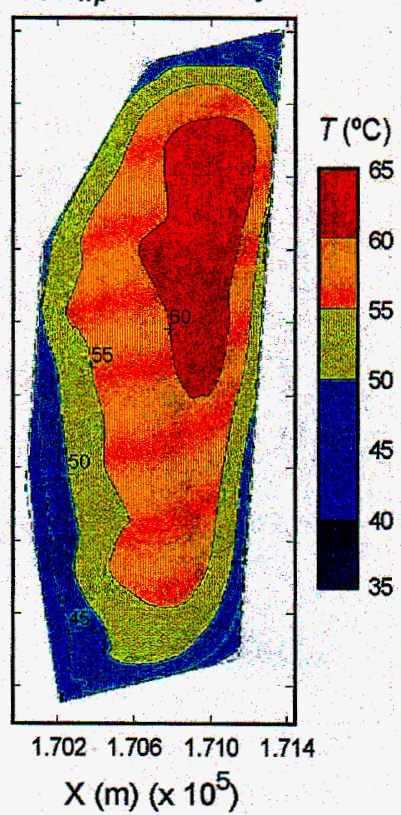

(c) $T_{w p}$ at $1000 \mathrm{yr}$

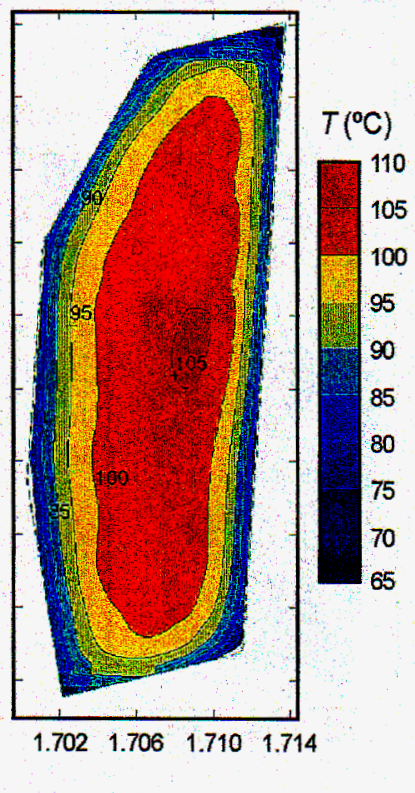

(f) $T_{w p}$ at $1,000,000 \mathrm{yr}$

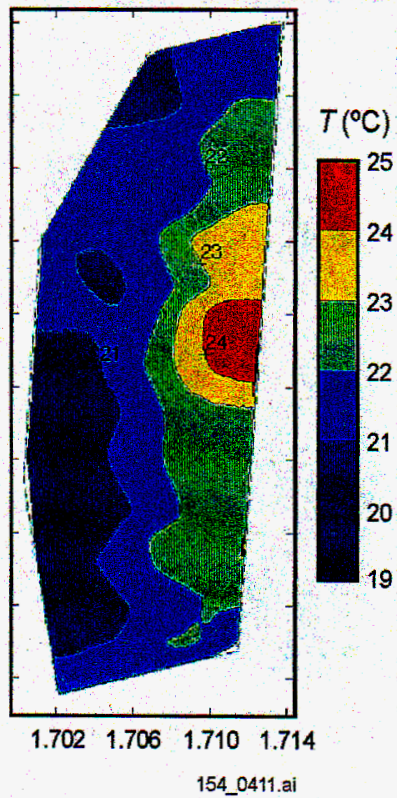

154_0411.ai

Source: Produced using files from Buscheck 2001 [DIRS 155449].

NOTE: Average 21-PWR waste package Mean-infiltration-flux scenario. $T_{W P}=$ waste package temperature,

$\mathrm{X}=$ east-west distances, $\mathrm{Y}=$ north-south distances.

Figure 57 Contour plots of waste-package temperature at six points in time in the HTOM repository. 
(a) $R H_{w p}$ at $50 \mathrm{yr}$

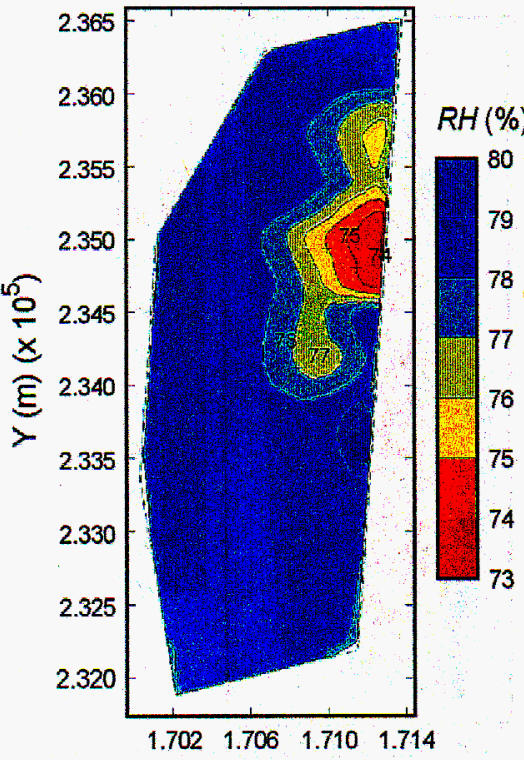

(d) $R H_{w p}$ at $2000 \mathrm{yr}$

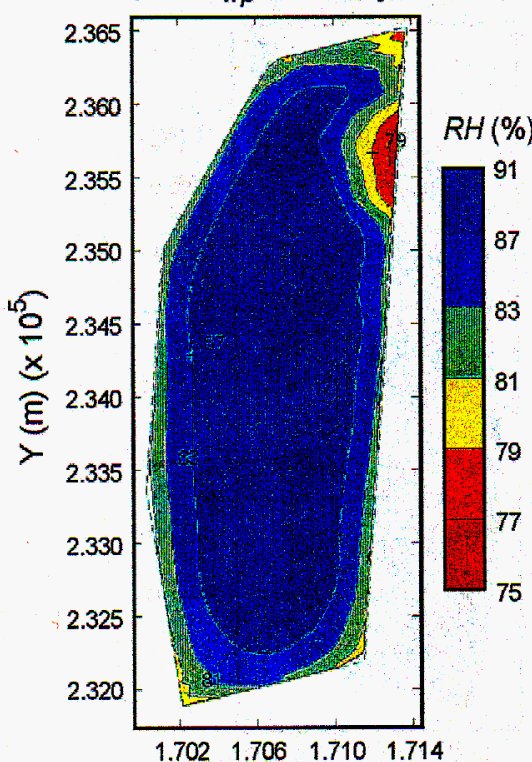

(b) $R H_{w p}$ at $60 \mathrm{yr}$

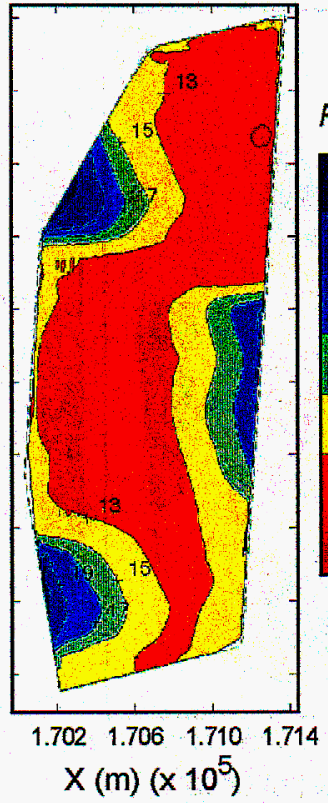

(e) $R H_{w p}$ at $10,000 \mathrm{yr}$

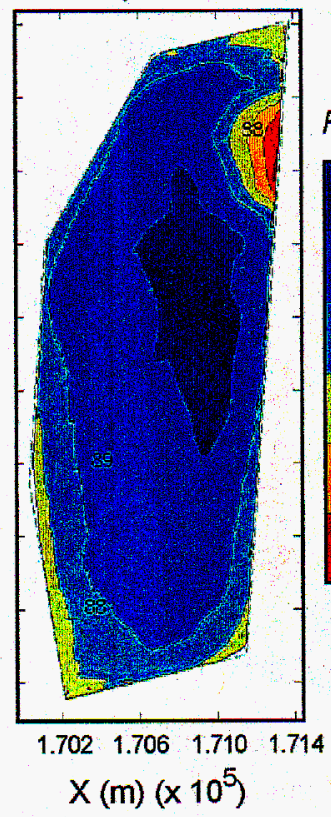

(c) $R H_{w p}$ at $1000 \mathrm{yr}$

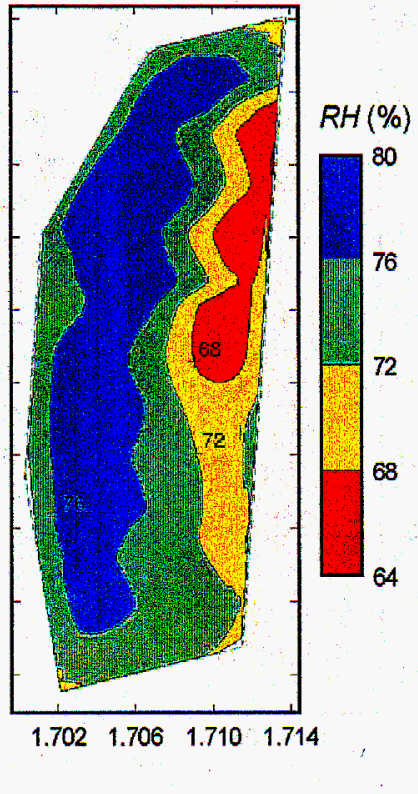

(f) $R H_{w p}$ at $1,000,000 \mathrm{yr}$

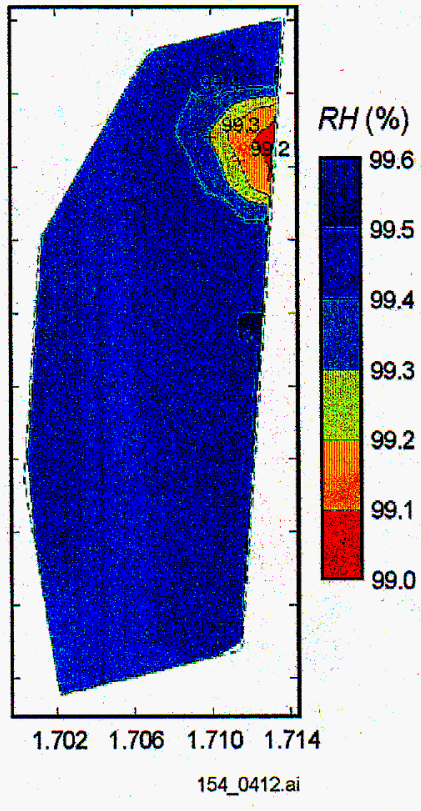

154_0412.ai

Source: Produced using files from Buscheck 2001 [DIRS 155449].

NOTE: Average 21-PWR waste package Mean-infiltration-flux scenario. $R H_{W P}=$ waste package relative humidity,

$\mathrm{X}=$ east-west distances, $\mathrm{Y}=$ north-south distances.

Figure 58 Contour plots of waste-package relative humidity at six points in time for the HTOM repository. 
(a) Waste Package

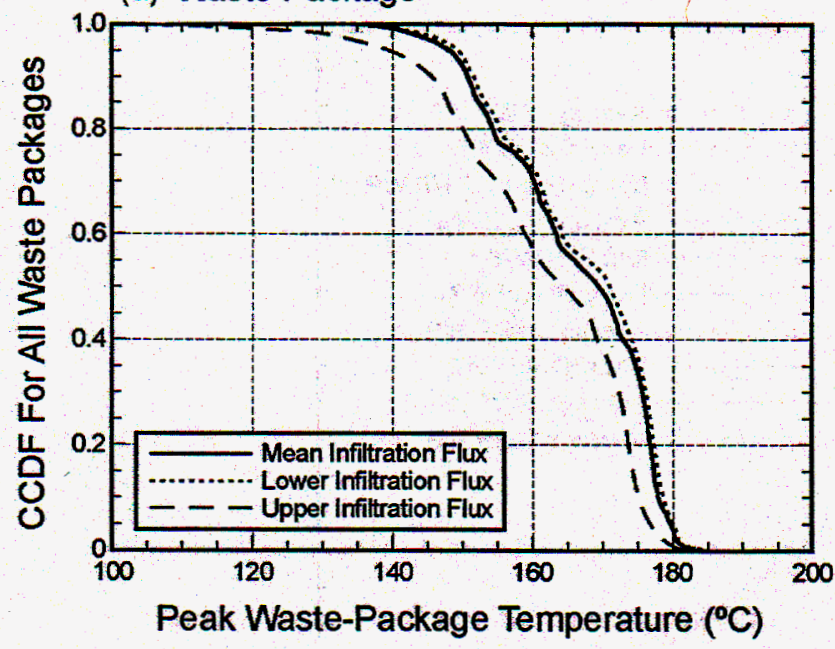

(c) Waste Package

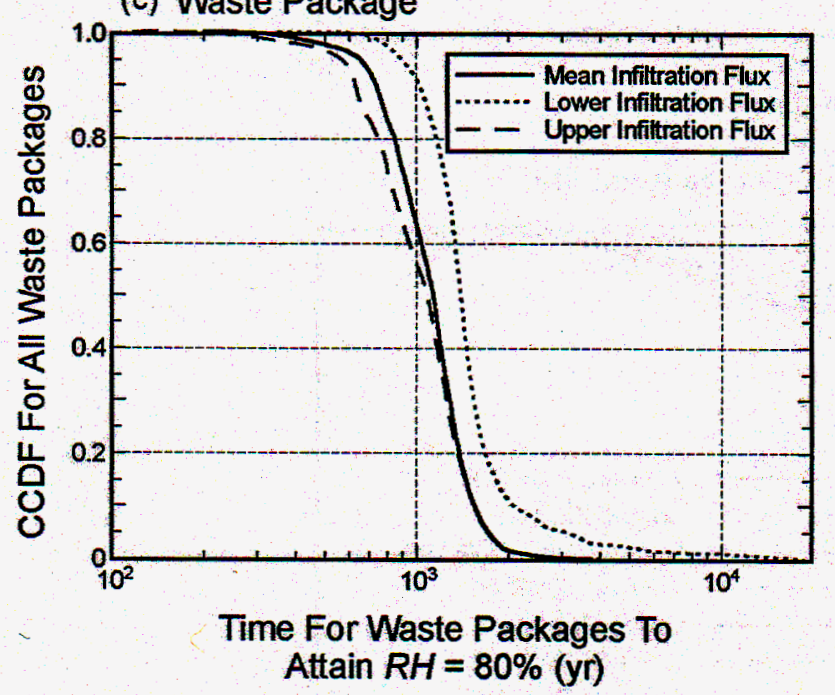

(b) Dritt Wall

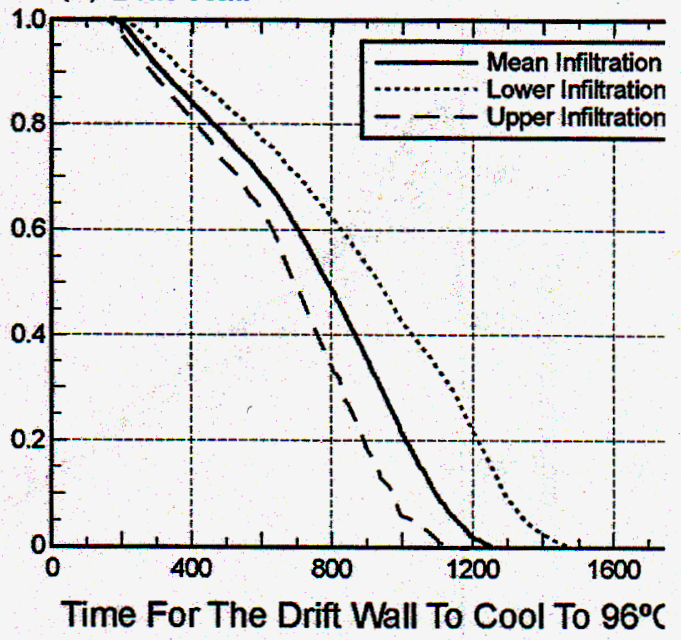

(d) Waste Package

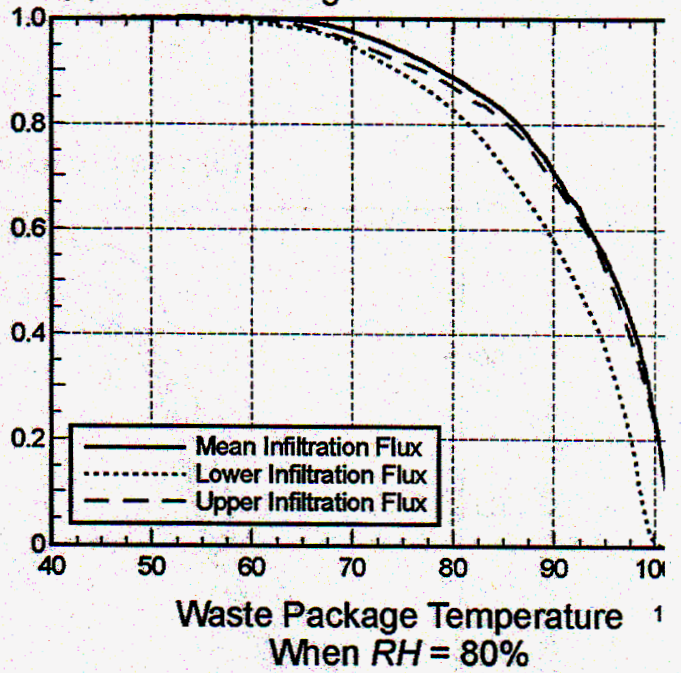

154_0413.ai

Source: Produced using files from Buscheck 2001 [DIRS 155449].

NOTE: $\mathrm{CCDF}=$ complementary cumulative distribution function.

Figure 59 Complementary cumulative distribution function of temperature and relative humidity conditions on the drift wall and the waste package for the HTOM repository. 
(a) waste-package temperature

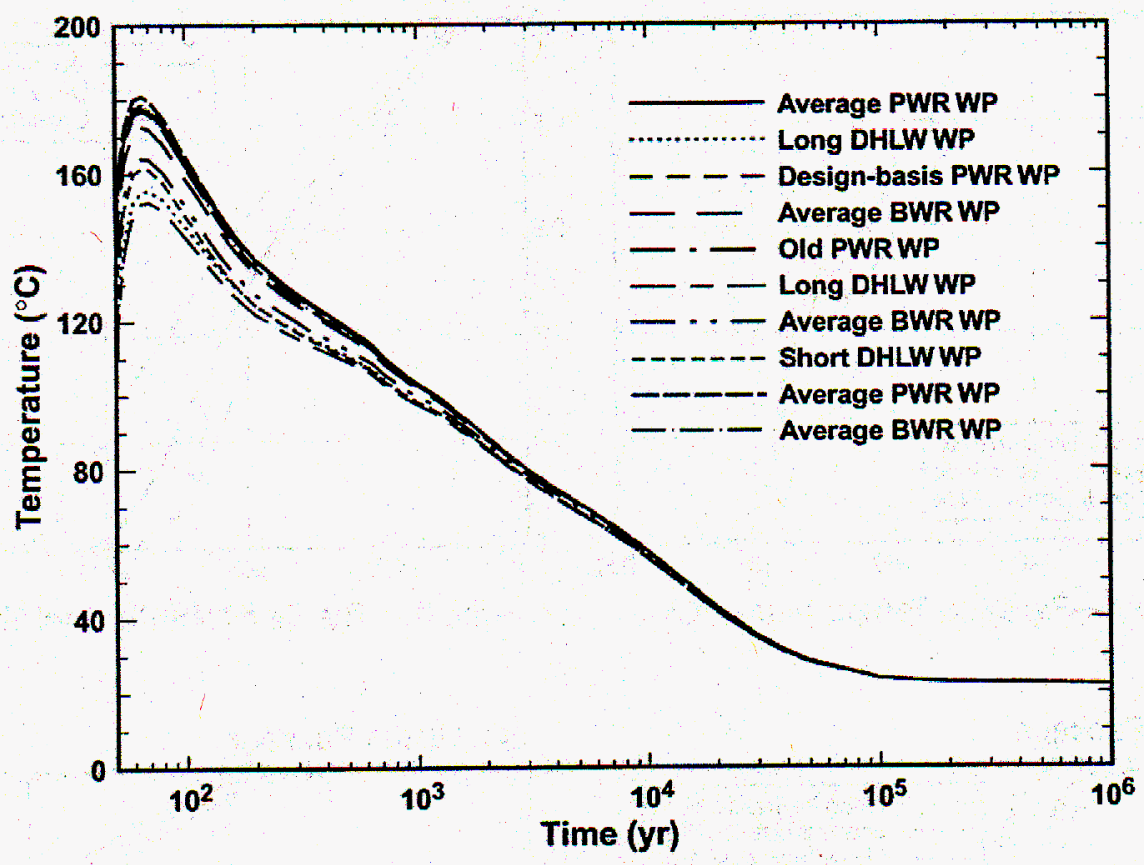

(b) waste-package relative humidity

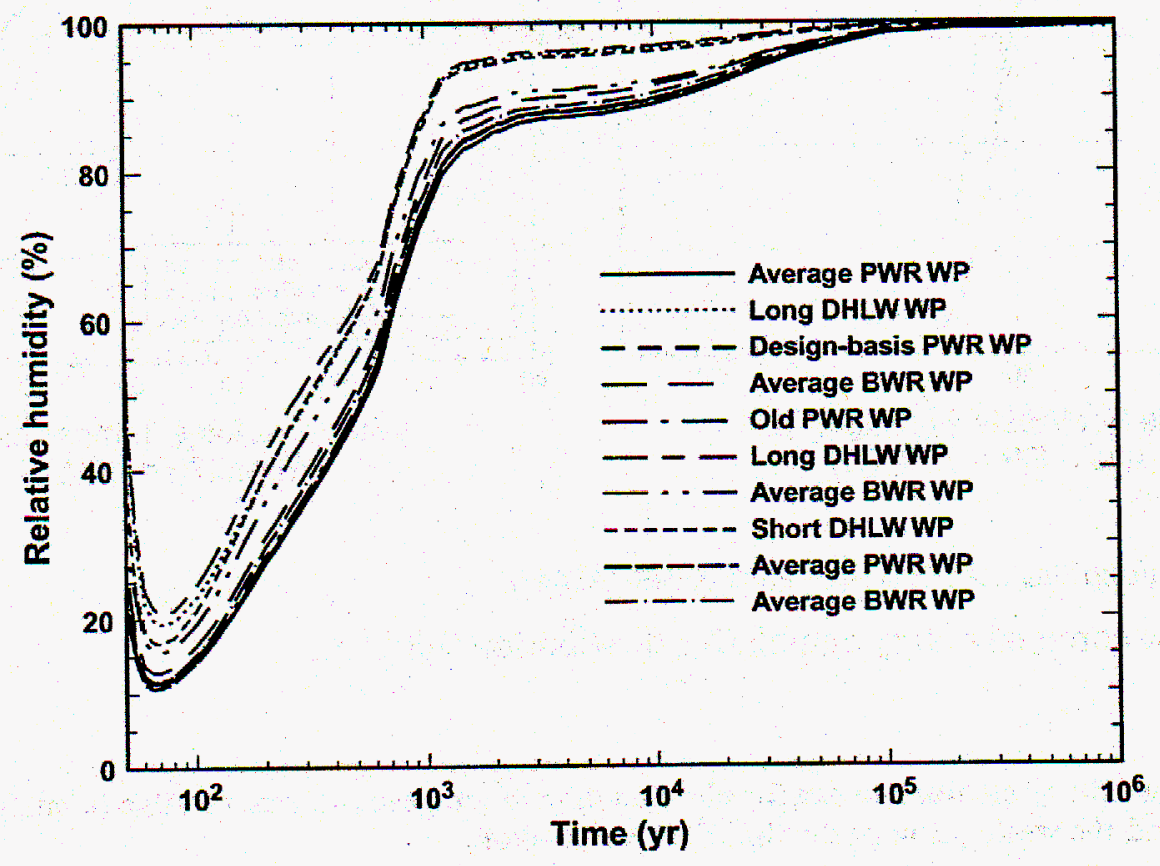

case1_micro

Source: Produced using files from Buscheck 2001 [DIRS 155449].

NOTE: Curves are for the 10 waste packages considered in MSTH model calculations at the L5C3 location and the mean-infiltration-flux scenario.

Figure 60 Temperature and relative humidity histories for the HTOM repository as predicted by the MSTH model. 


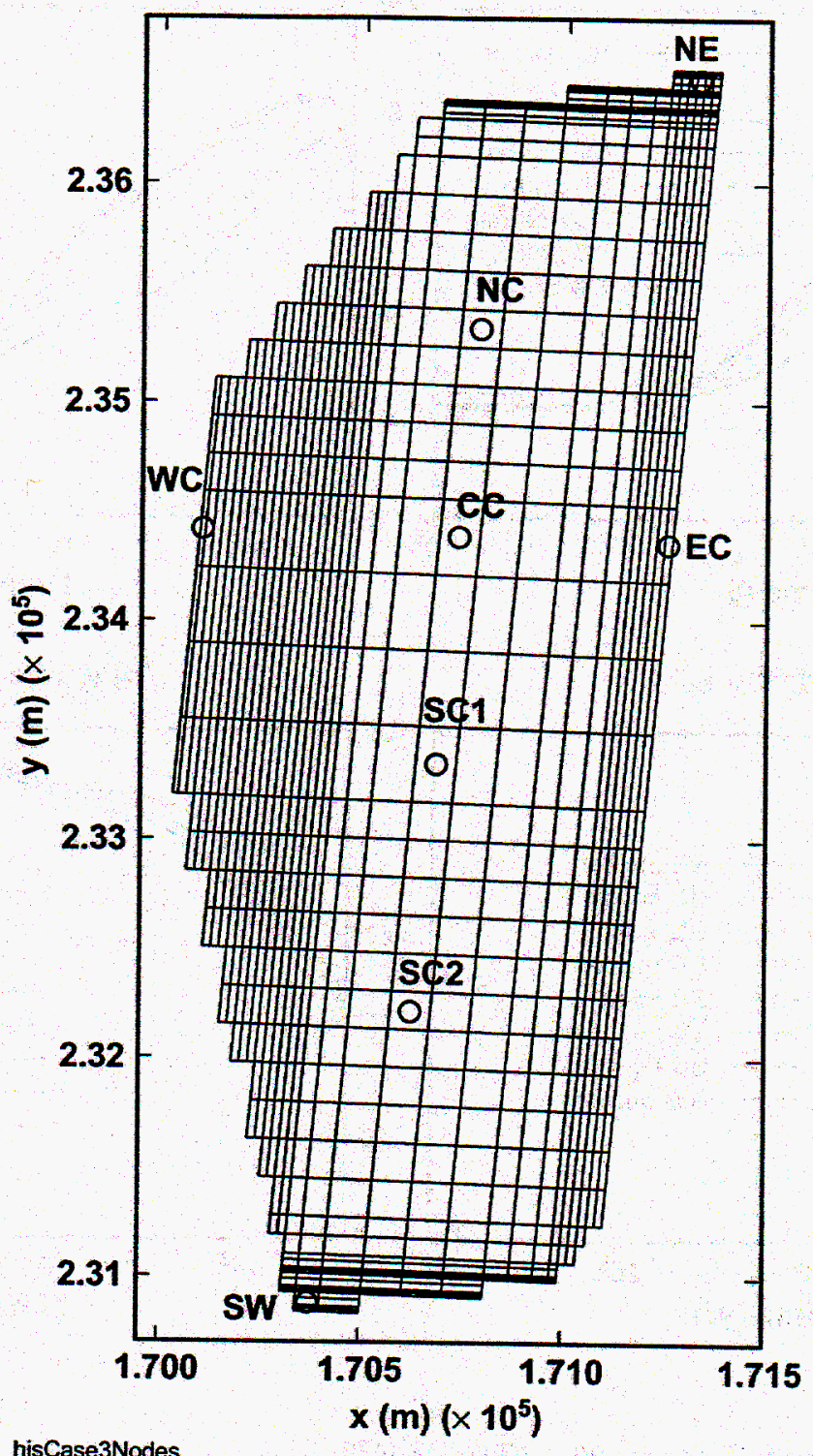

Source: Produced using files from Buscheck 2001 [DIRS 155449].

NOTE: Plan view of the potential lower-temperature operating mode repository showing the geographic locations considered in various analyses. $\mathrm{X}=$ east-west distances, $\mathrm{Y}=$ north-south distances.

Figure 61 Location of the eight sites used in analysis of LTOM repository. 
(a) drift wall

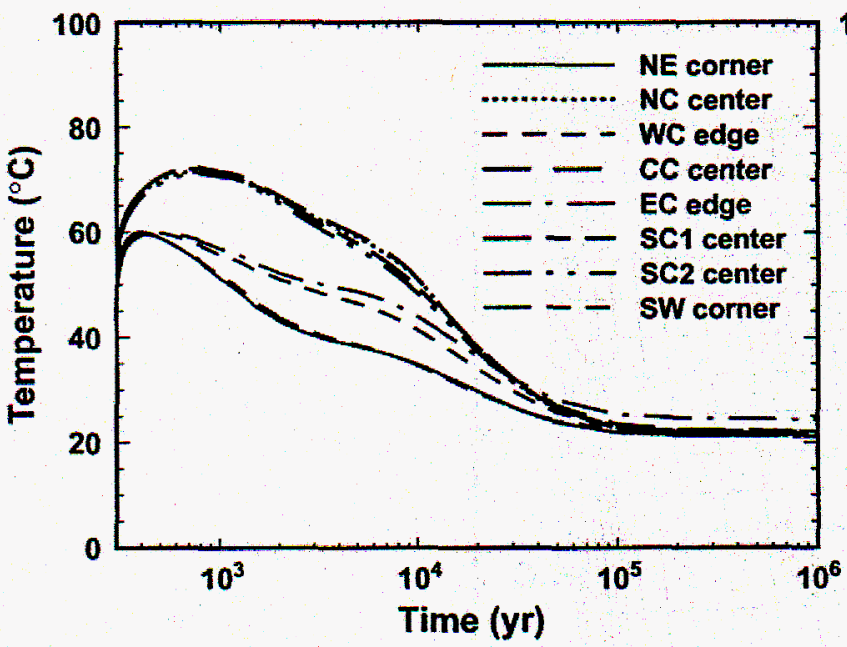

(c) drift wall

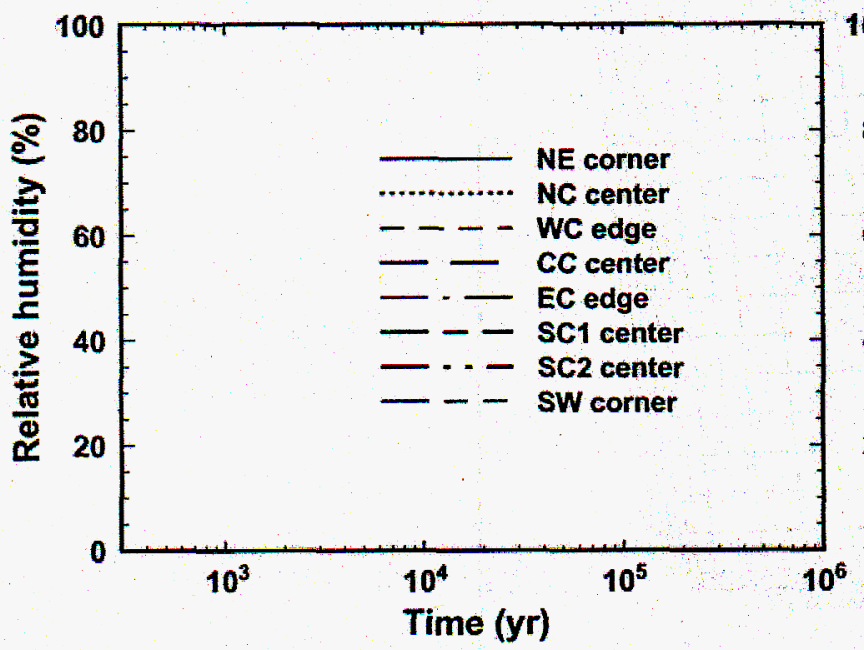

(b) waste package

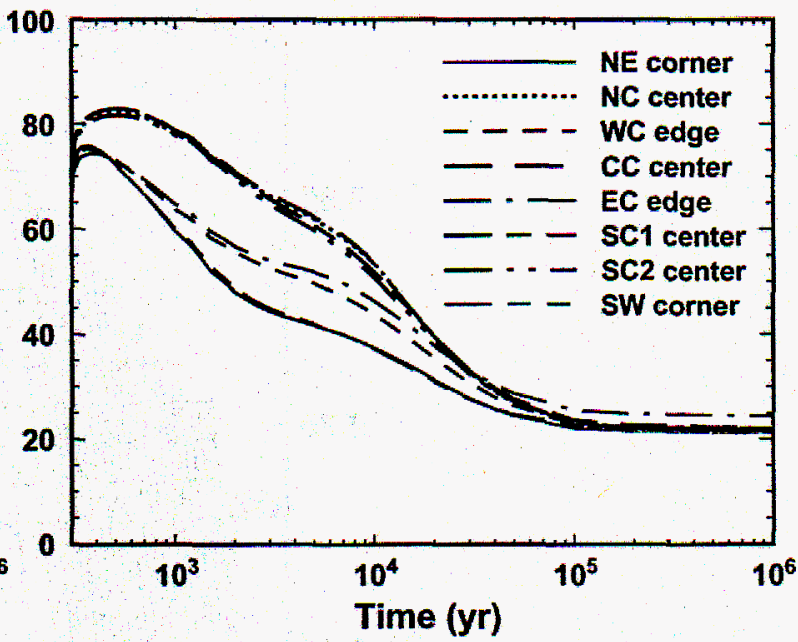

(d) waste package

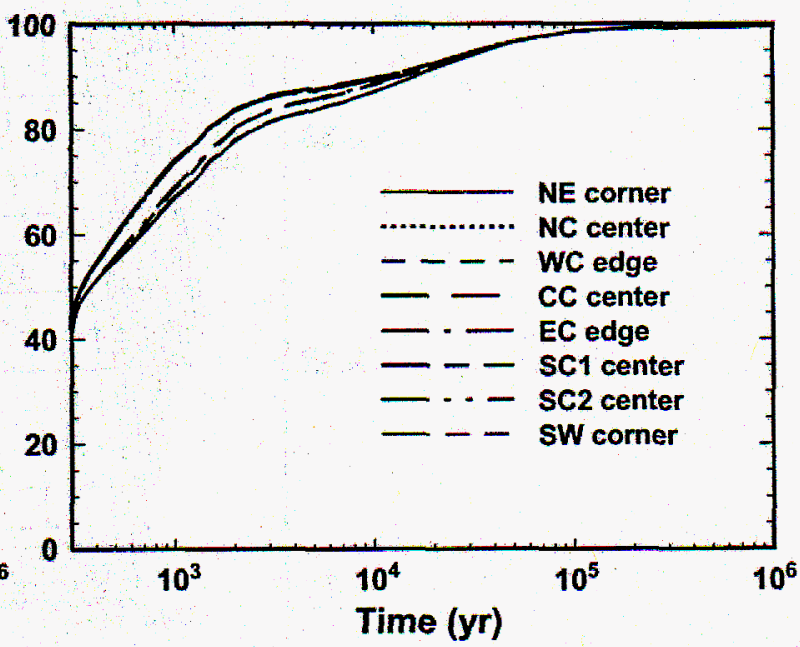

case3_mi_T-RH_8loc

Source: Produced using files from Buscheck 2001 [DIRS 155449].

NOTE: Average 21-PWR CSNF waste package Mean-infiltration-flux scenario.

Figure 62 Temperature and relative humidity histories on the drift wall and the waste package for the eight locations in the LTOM repository. 
(a) invert

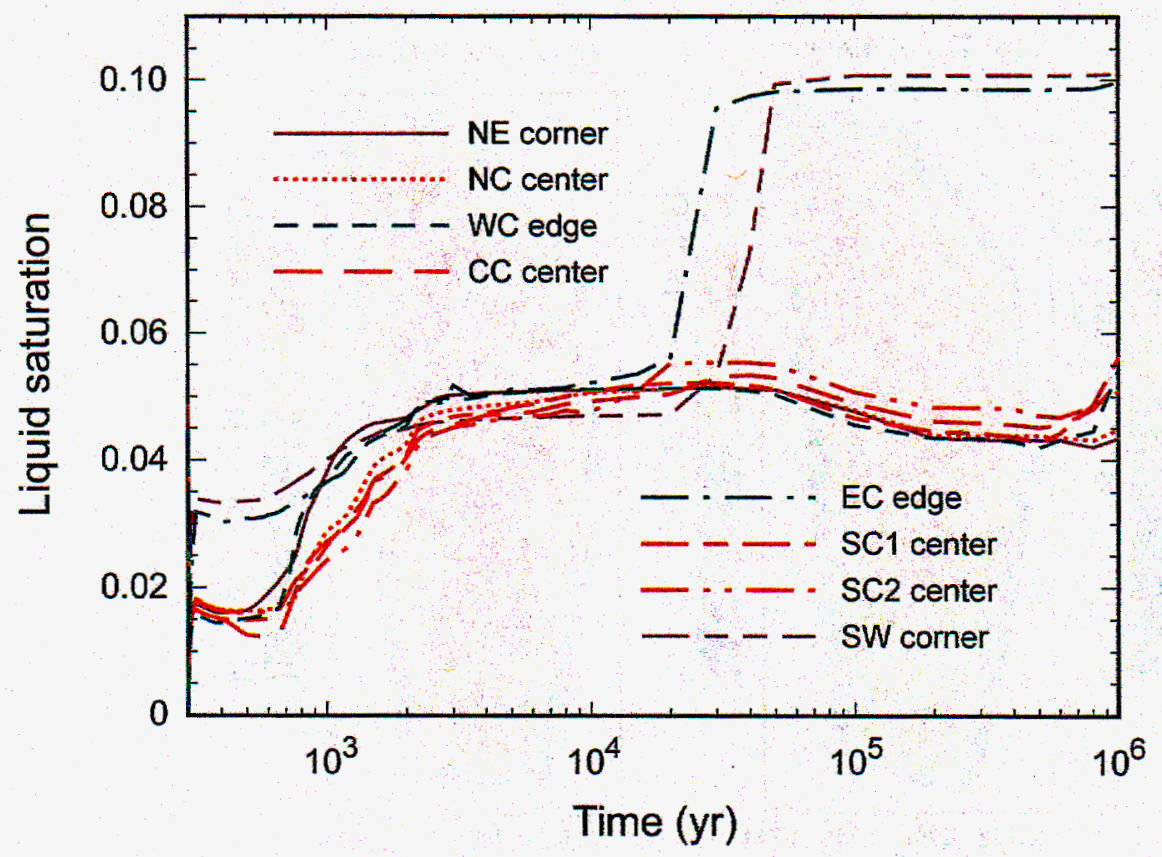

(b) invert

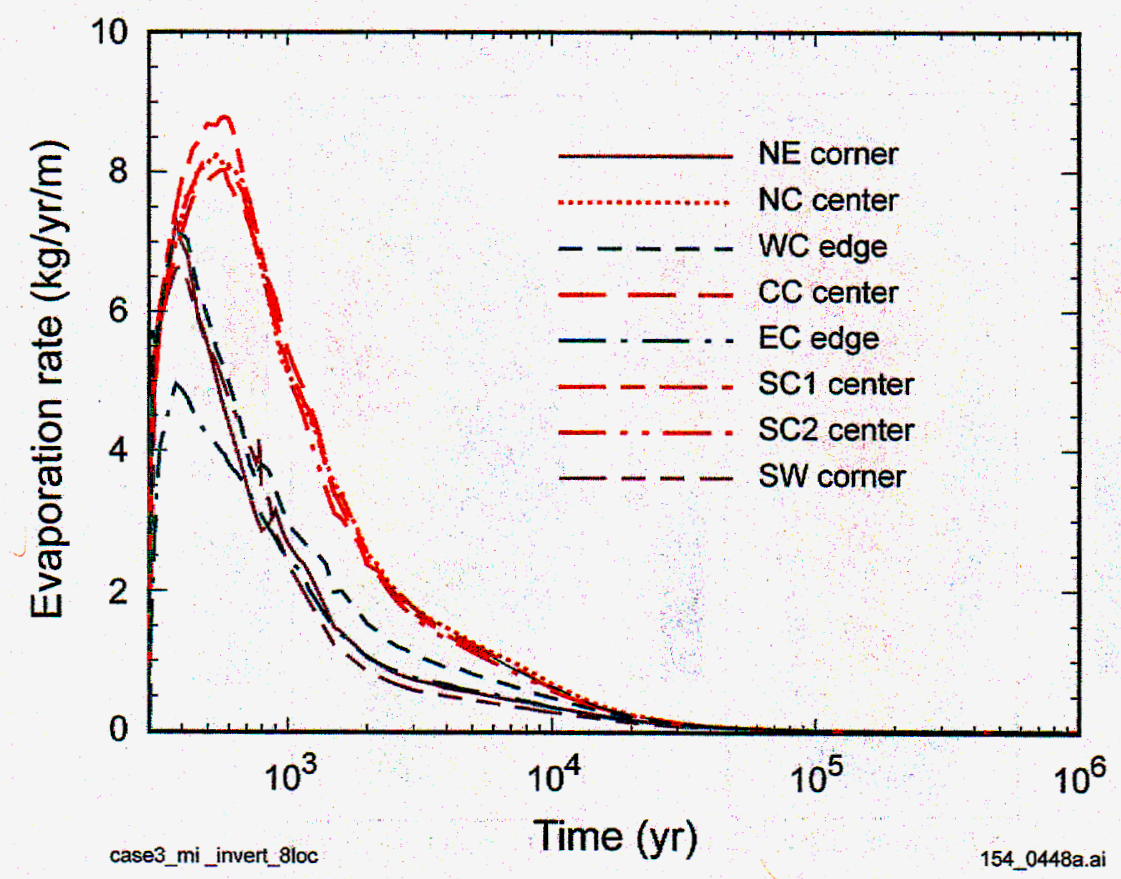

Source: Produced using files from Buscheck 2001 [DIRS 155449].

NOTE: Average 21-PWR CSNF waste Mean-infiltration-flux scenario.

Figure 63 Liquid saturation and evaporation rate histories for the eight locations in the potential LTOM repository. 
(a) $T_{\mathrm{wp}}$ at $300 \mathrm{yr}$

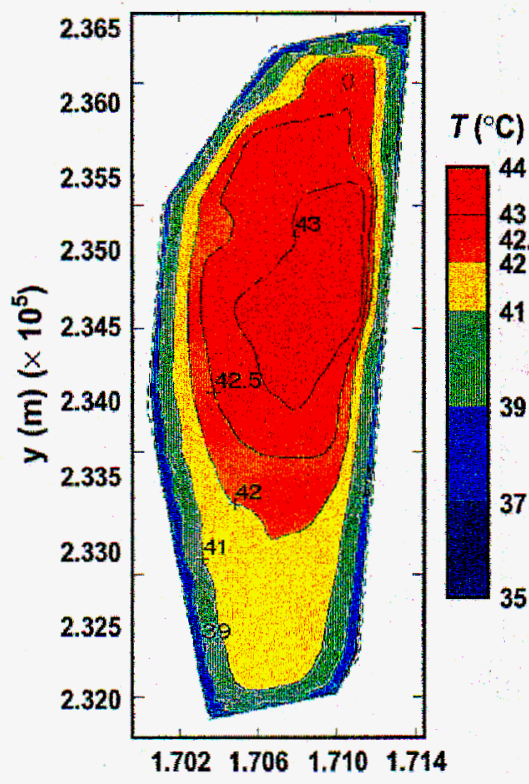

(d) $T_{\text {wp }}$ at $2000 \mathrm{yr}$

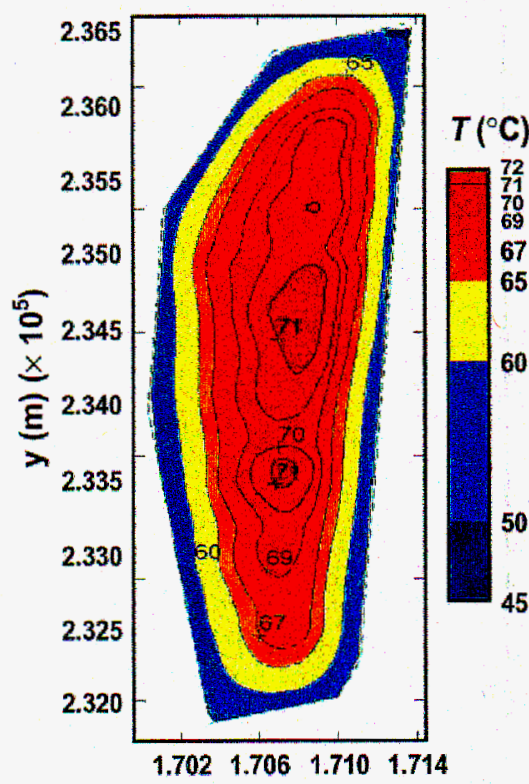

(b) $T_{\text {wp }}$ at $400 \mathrm{yr}$

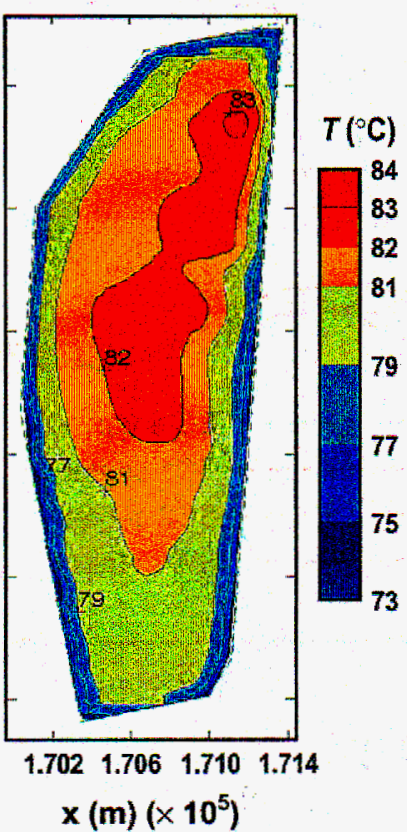

(e) $T_{\text {wp }}$ at $10,000 \mathrm{yr}$

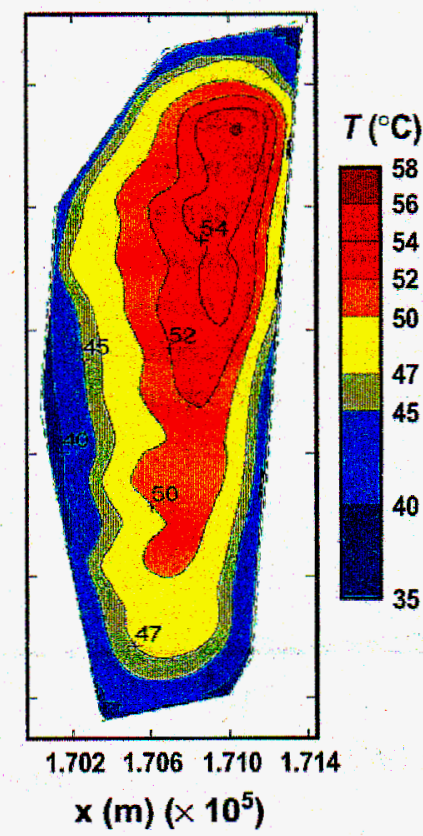

(c) $T_{\text {wp }}$ at $1000 \mathrm{yr}$

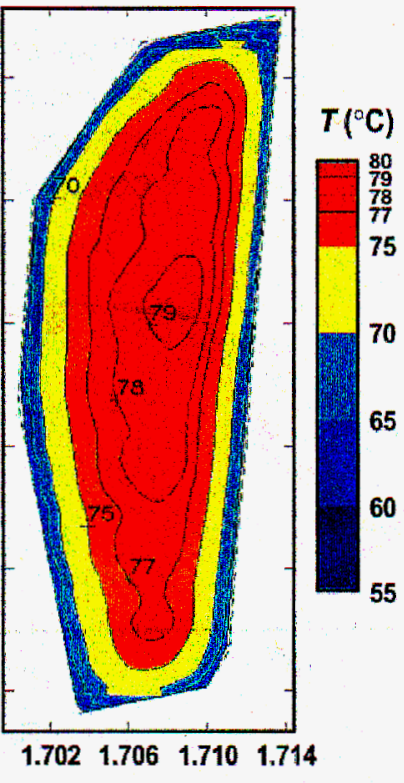

(f) $T_{\mathrm{wp}}$ at $1,000,000 \mathrm{yr}$

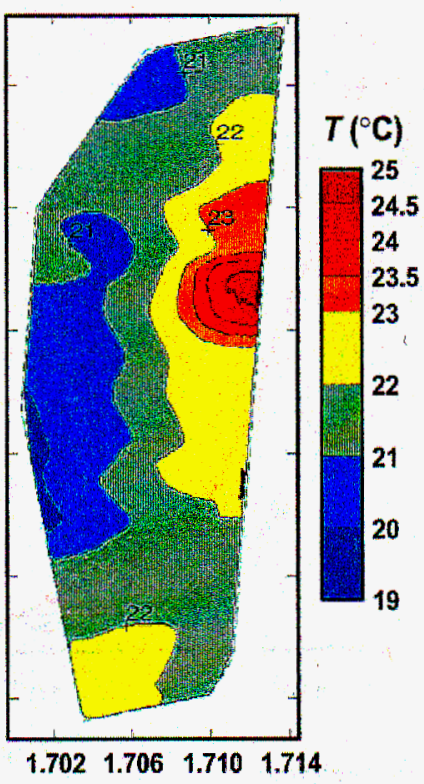

Twp03mi

Source: Produced using files from Buscheck 2001 [DIRS 155449].

NOTE: Average 21-PWR waste package Mean-infiltration-flux scenario. $R H_{W P}=$ waste package relative humidity, $\mathrm{X}=$ east-west distances, $\mathrm{Y}=$ north-south distances.

Figure 64 Contour plots of waste-package temperature at six points in time in the LTOM repository. 
(a) $R H_{\mathrm{wp}}$ at $300 \mathrm{yr}$

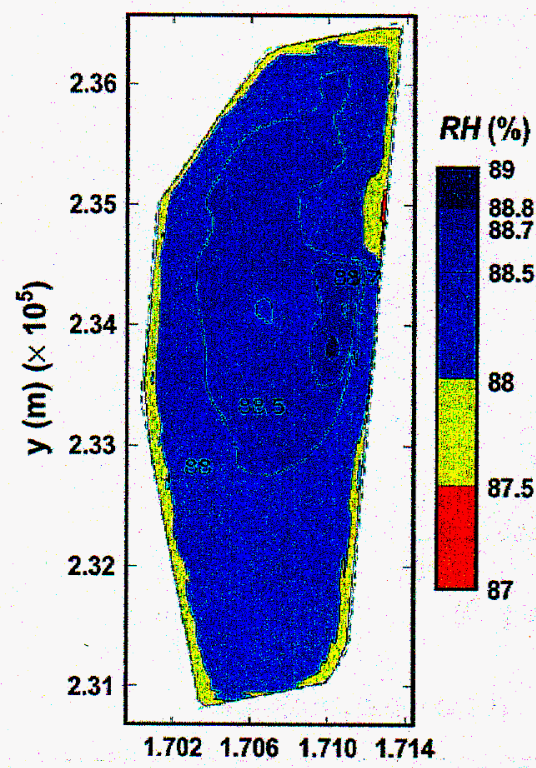

(d) $R H_{\text {wp }}$ at $2000 \mathrm{yr}$

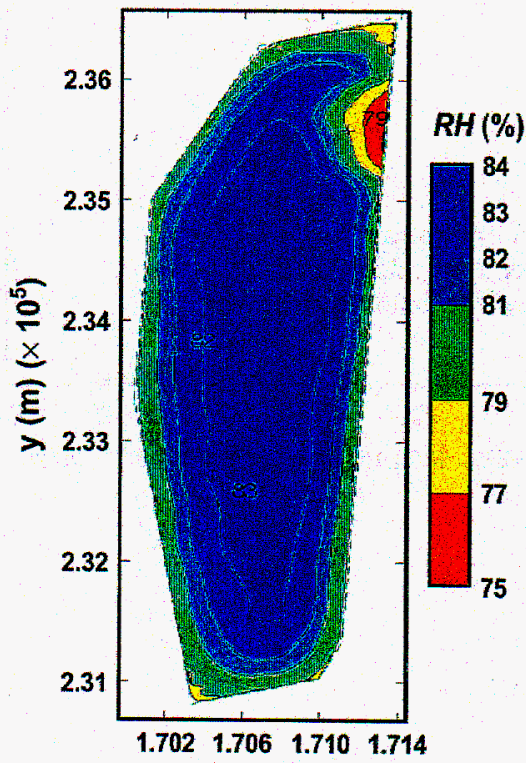

RHwp03mi (b) $R H_{\text {wp }}$ at $400 \mathrm{yr}$

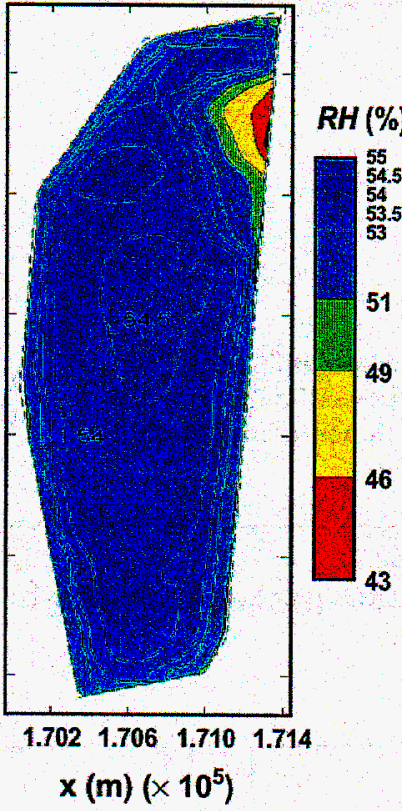

(e) $R H_{\text {wp }}$ at $10,000 \mathrm{yr}$

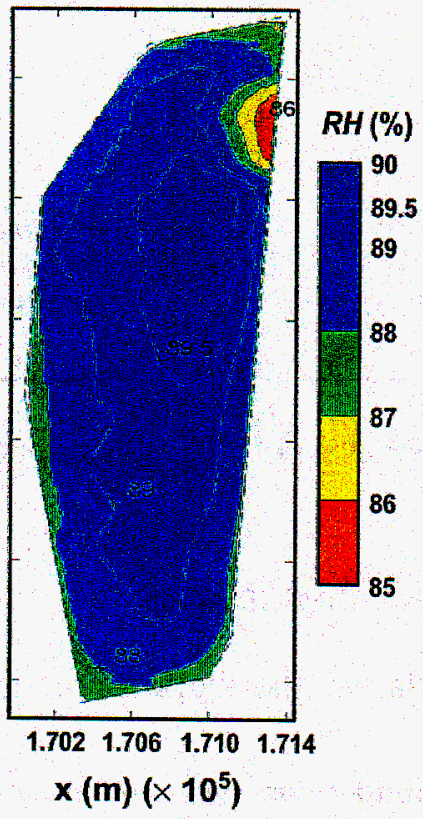

(c) $R H_{w p}$ at $1000 \mathrm{yr}$

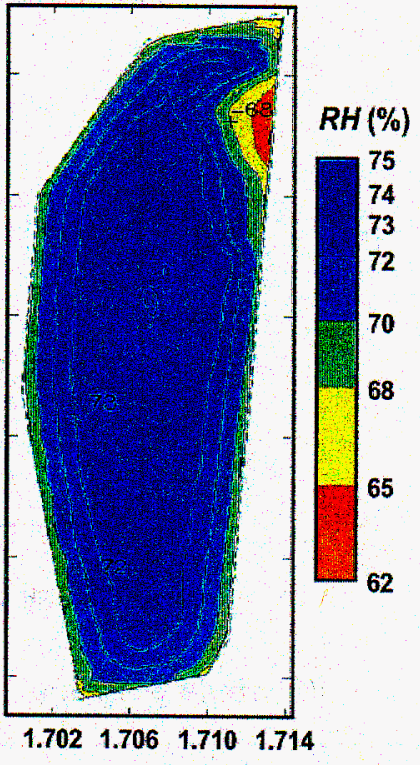

(f) $R H_{\text {wp }}$ at $1,000,000 \mathrm{yr}$

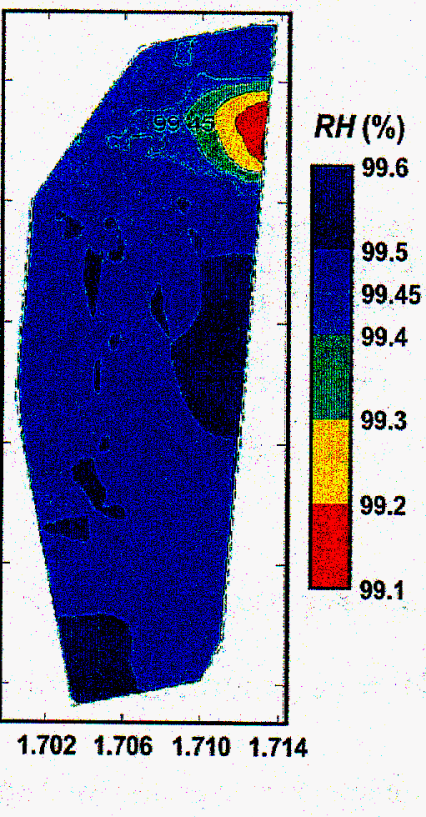

Source: Produced using files from Buscheck 2001 [DIRS 155449].

NOTE: Average 21-PWR waste package Mean-infiltration-flux scenario. $R H_{W P}=$ waste package relative humidity, $\mathrm{X}=$ east-west distances, $\mathrm{Y}=$ north-south distances.

Figure 65 Contour plots of waste-package relative humidity at six points in time for the LTOM repository. 
(a) waste package

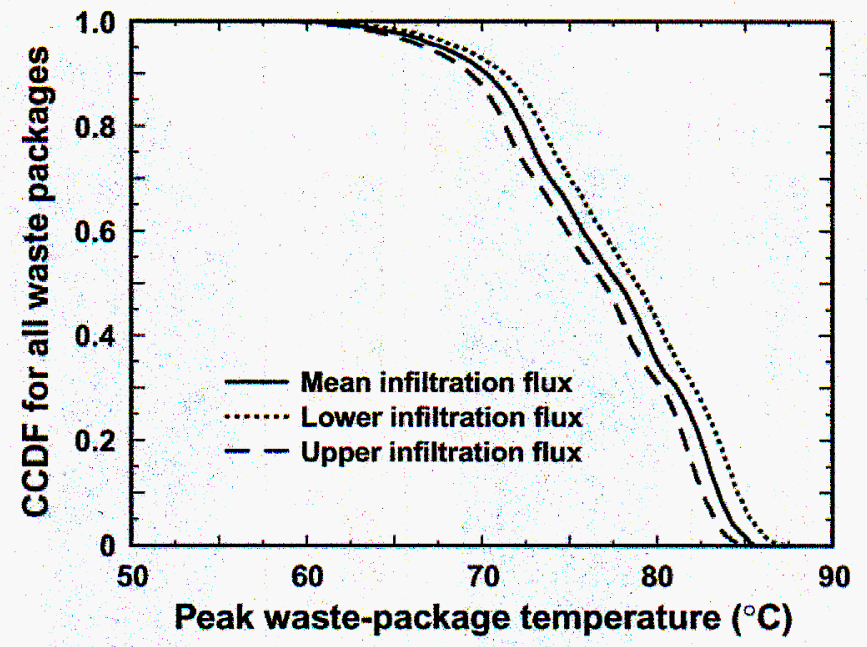

(b) waste package

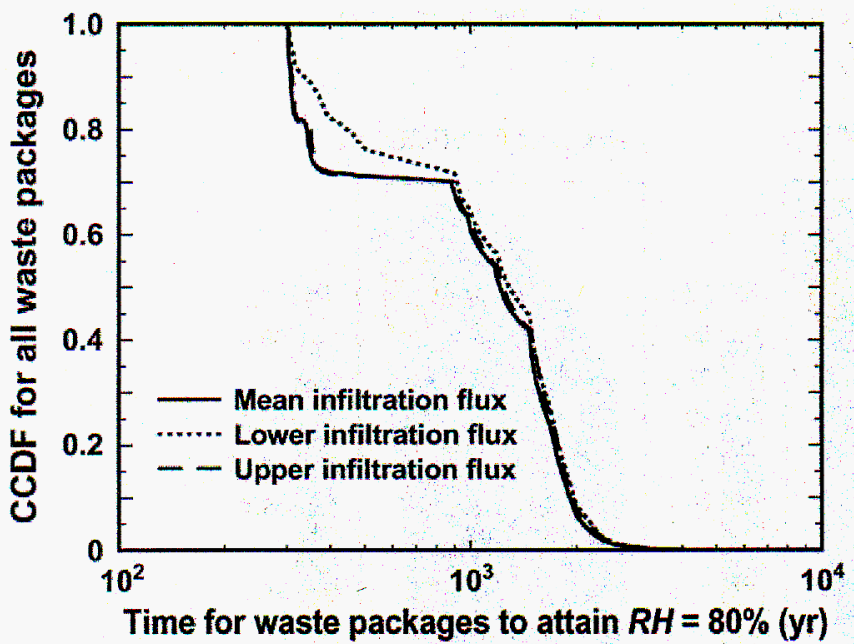

(c) waste package

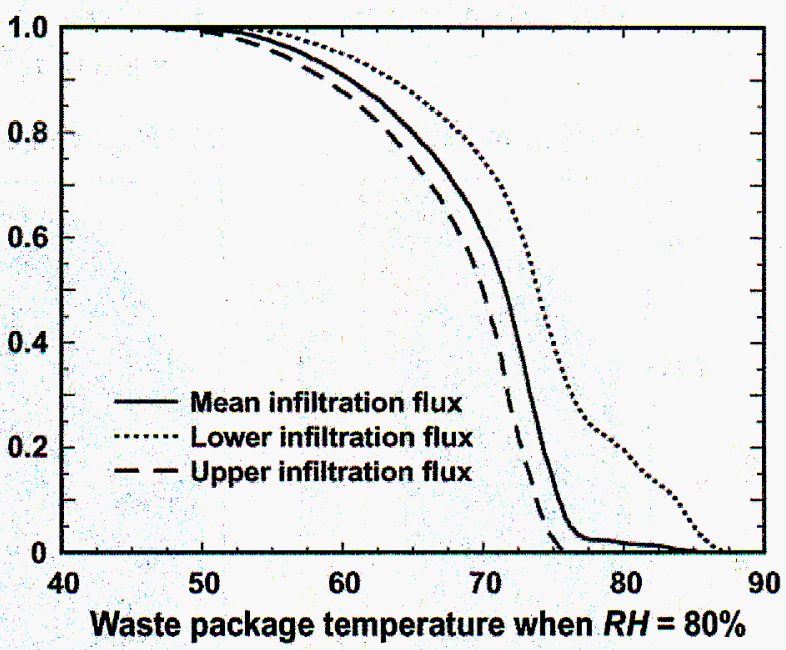

case3_CCDF_wp_mlu

Source: Produced using files from Buscheck 2001 [DIRS 155449].

NOTE: $\mathrm{CCDF}=$ complementary cumulative distribution function.

Figure 66 Complementary cumulative distribution function of temperature and relative humidity conditions on the waste package for the LTOM repository. 
(a) waste-package temperature

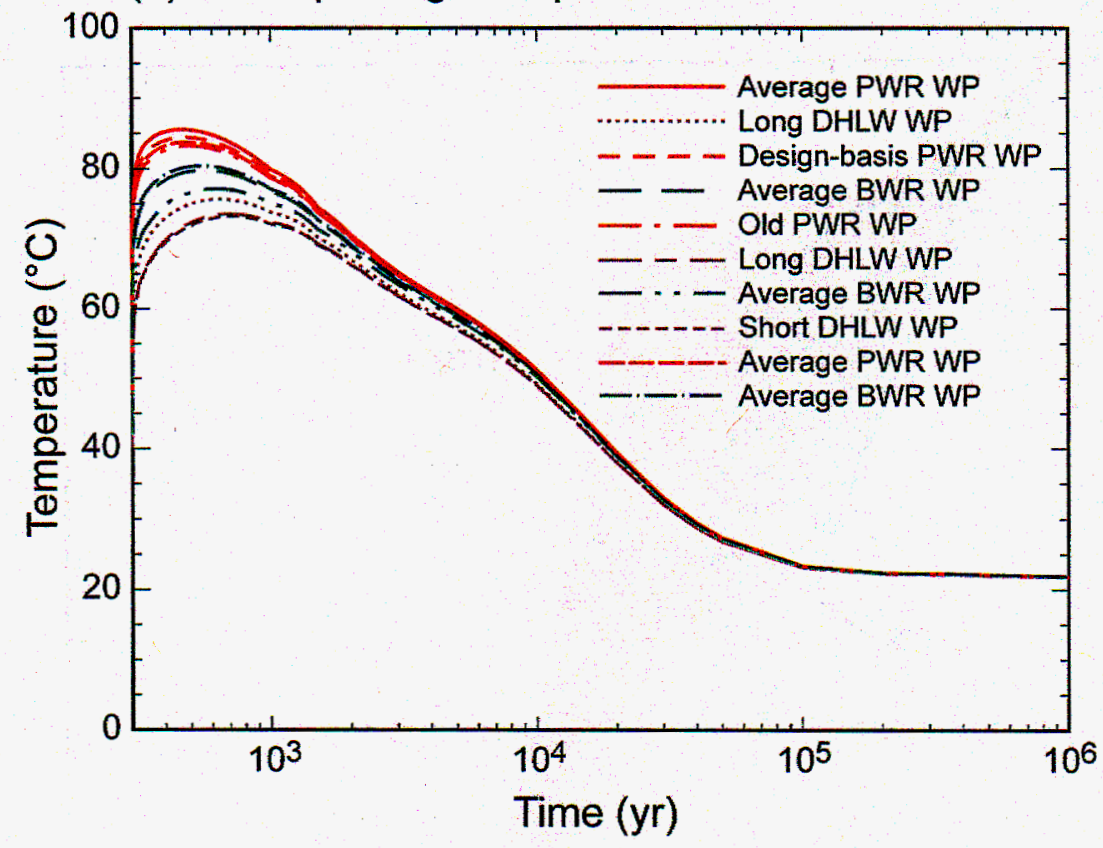

(b) waste-package relative humidity

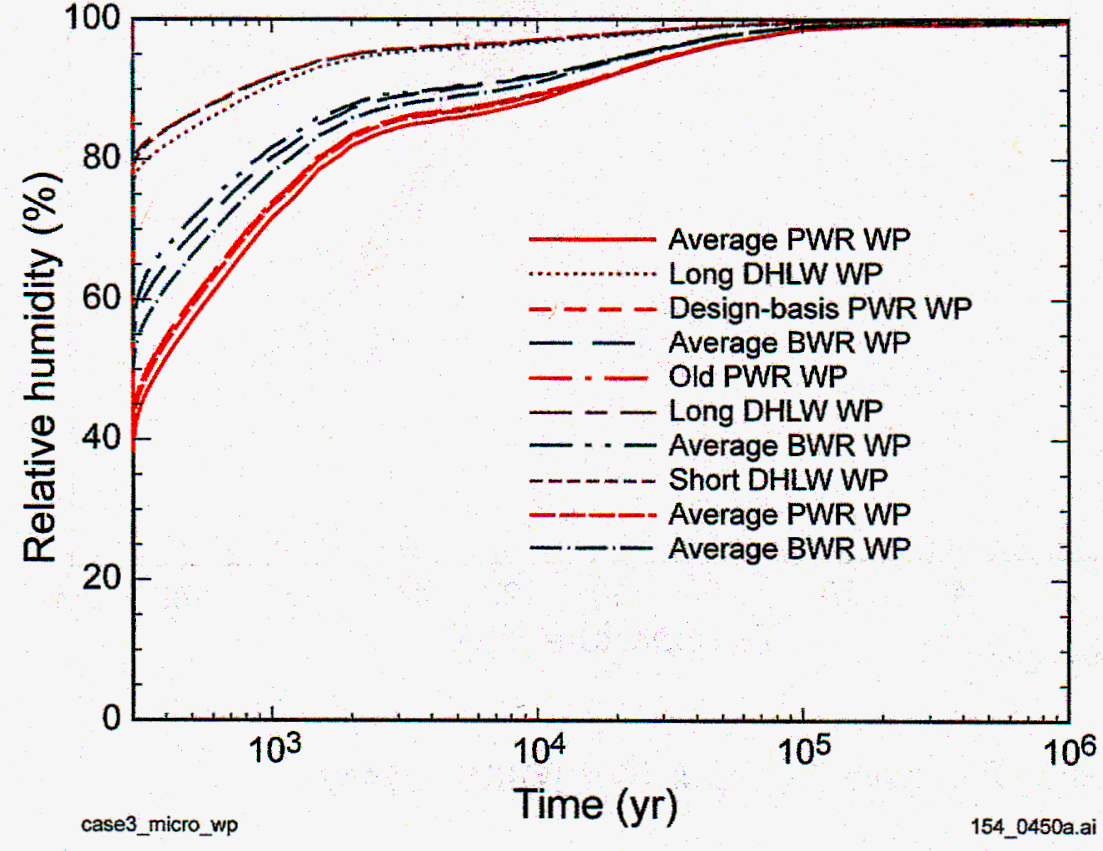

154_0450a.ai

Source: Produced using files from Buscheck 2001 [DIRS 155449].

NOTE: Curves are for the 10 waste packages considered in the MSTH model calculations at the L5C3 location for the Mean-infiltration-flux scenario.

Figure 67 Temperature and relative humidity histories for the LTOM repository as predicted by the MSTH model. 


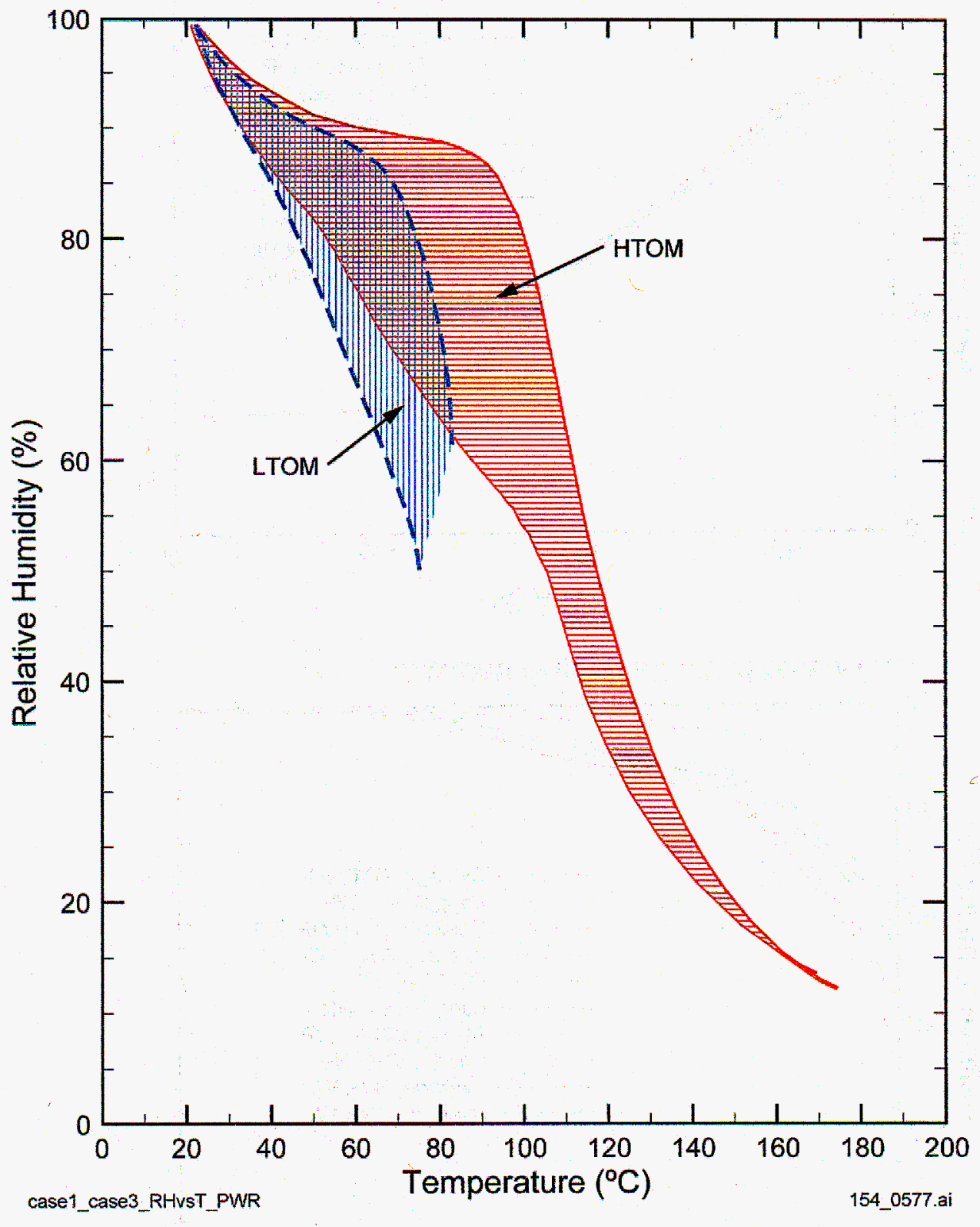

154_0577.ai

Source: Produced using files from Buscheck 2001 [DIRS 155449].

Figure 68 Thermohydrologic parameter evolution for the HTOM and the LTOM repositories. 


\section{TABLES}

Table 1 Design Parameters for Sensitivity Studies to Address Two Operational Modes: HTOM and LTOM.

\begin{tabular}{|l|l|l|}
\hline \multicolumn{1}{|c|}{ Design feature } & \multicolumn{1}{|c|}{$\begin{array}{c}\text { Higher-Temperature } \\
\text { Operation Mode } \\
\text { (HTOM: above boiling } \\
\text { repository) }\end{array}$} & \multicolumn{1}{|c|}{$\begin{array}{c}\text { Lower-Temperature } \\
\text { Operating Mode (LTOM: } \\
85^{\circ} \mathrm{C} \text { Waste Package) }\end{array}$} \\
\hline Drift spacing & $81 \mathrm{~m}$ & $81 \mathrm{~m}$ \\
\hline Areal Mass Loading & $54.5 \mathrm{MTU} / \mathrm{acre}$ & $45.7 \mathrm{MTU} / \mathrm{acre}$ \\
\hline Repository footprint & $4.65 \mathrm{~km}^{2}$ & $5.58 \mathrm{~km}$ \\
\hline Lineal Power density & $1.35 \mathrm{~kW} / \mathrm{m}$ & $1.13 \mathrm{~kW} / \mathrm{m}$ \\
\hline Length of Ventilation & $50 \mathrm{yrs}$ forced & $300 \mathrm{yrs}$ forced \\
\hline Heat removal by Ventilation & $70 \%$ & $80 \%$ \\
\hline $\begin{array}{l}\text { Waste Package } \\
\text { configuration/spacing }\end{array}$ & Line load, $10 \mathrm{~cm}$ & $\begin{array}{l}\text { Line/point load, gaps from } \\
10 \mathrm{~cm} \text { to } 2.8 \mathrm{~m}\end{array}$ \\
\hline
\end{tabular}


Table 2 Calculation inputs and sources.

\begin{tabular}{|c|c|c|}
\hline $\begin{array}{l}\text { DATA STORE } \\
\text { and reference to } \\
\text { Sub-flowchart }\end{array}$ & Input Description & DTN or Document Number \\
\hline $\begin{array}{l}\text { DATA STORE A } \\
\text { Sub-flowcharts } \\
1,2,3,4,9\end{array}$ & $\begin{array}{l}.3-D \text { UZ MODEL GRIDS FOR CALCULATION OF } \\
\text { FLOW FIELDS FOR PA: ONE PRIMARY SINGLE- } \\
\text { CONTINUUM MESH AND THREE DUAL-CONTINUA } \\
\text { MESHES USED TO GENERATE 3-D UNSATURATED } \\
\text { ZONE FLOW FIELDS FOR PERFORMANCE } \\
\text { ASSESSMENT. ANL-NBS-HS-000015, } \\
\text { MOL. } 19990721.0517 \text {. SRLA SUPPORTING DATA. 24- } \\
\text { sep-1999 }\end{array}$ & LB990701233129.001 \\
\hline $\begin{array}{l}\text { DATA STORE B } \\
\text { Sub-flowcharts } 1,12\end{array}$ & $\begin{array}{l}\text { TABULATED IN-DRIFT GEOMETRIC AND THERMAL } \\
\text { PROPERTIES USED IN DRIFT-SCALE MODELS FOR } \\
\text { TSPA-SR (TOTAL SYSTEM PERFORMANCE } \\
\text { ASSESSMENT-SITE RECOMMENDATION).: 30-aug- } \\
1999\end{array}$ & SN9908T0872799.004 \\
\hline $\begin{array}{l}\text { DATA STORE C } \\
\text { Sub-flowcharts } 2,3\end{array}$ & $\begin{array}{l}\text { HTOM: BSC (Bechtel SAIC Company) 2001. Site } \\
\text { Recommendation Subsurface Layout. ANL-SFS-MG- } \\
000001 \text { REV 00 ICN 02. Las Vegas, Nevada: Bechtel } \\
\text { SAIC Company. ACC: MOL.2.0010411.0131 } \\
\\
\text { LTOM: BSC (Bechtel SAIC Company) 2001. Lower- } \\
\text { Temperature Subsurface Layout and Ventilation Concepts. } \\
\text { ANL-WER-MD-000002 REV 00. Las Vegas, Nevada: } \\
\text { Bechtel SAIC Company. ACC: MOL.20010718.0225. }\end{array}$ & $\begin{array}{l}\text { ANL-SFS-MG-000001 REV } 00 \text { ICN } 02 \\
\text { (MOL.2.0010411.0131) } \\
\text { ANL-WER-MD-000002 REV } 00 \\
\text { (MOL.20010718.0225) }\end{array}$ \\
\hline $\begin{array}{l}\text { DATA STORE D } \\
\text { Sub-flowcharts } \\
5,6,7,16\end{array}$ & $\begin{array}{l}\text { BSC (Bechtel SAIC Company) 2001. Design Input for the } \\
\text { Engineered Barrier System Environment and Barriers. } \\
\text { Input Transmittal 00422.Ta. Las Vegas, Nevada: Bechtel } \\
\text { SAIC Company. ACC: MOL.20010405.0196. }\end{array}$ & $\begin{array}{l}00422 . \mathrm{Ta} \\
\text { (MOL.20010405.0196) }\end{array}$ \\
\hline $\begin{array}{l}\text { DATA STORE E } \\
\text { Sub-flowchart } 8\end{array}$ & $\begin{array}{l}\text { FLOW FIELD } \# 1,3,5,7,9,11,13,15,17 \text { : PRESENT DAY LOW, } \\
\text { MEAN, UPPER INFILTRATION MAP, GLACIAL LOW, } \\
\text { MEAN, UPPER INFILTRATION MAP, MONSOON LOW, } \\
\text { MEAN, UPPER INFILTRATION MAP FOR FLOW-THROUGH } \\
\text { PERCHED-WATER CONCEPTUAL MODEL. AMR U0050, } \\
\text { "UZ FLOW MODELS AND SUBMODELS," MDL-NBS-HS- } \\
\text { 000006, MOL.19990721.0527. SR/LA SUPPORTING DATA. }\end{array}$ & $\begin{array}{l}\text { LB990801233129.001 } \\
\text { LB990801233129.003 } \\
\text { LB990801233129.005 } \\
\text { LB990801233129.007 } \\
\text { LB990801233129.009 } \\
\text { LB990801233129.011 } \\
\text { LB990801233129.013 } \\
\text { LB990801233129.015 } \\
\text { LB990801233129.017 }\end{array}$ \\
\hline
\end{tabular}




\begin{tabular}{|l|l|l}
\hline DATA STORE F & $\begin{array}{l}\text { INPUT AND OUTPUT FILES OF NUMERICAL } \\
\text { SIMULATIONS OF THE MOUNTAIN-SCALE } \\
\text { THERMAL-HYDROLOGIC (TH) MODEL. AMR U0105, } \\
\text { "MOUNTAIN-SCALE COUPLED PROCESSES (TH) } \\
\text { MODELS," MDL-NBS-HS-000007, MOL.19990721.0528. } \\
\text { SR/LA SUPPORTING DATA. 11-mar-2000 }\end{array}$ & LB991201233129.001 \\
\hline DATA STORE G & $\begin{array}{l}\text { DRIFT SCALE CALIBRATED 1-D PROPERTY SET, } \\
\text { FY99: BASECASE INFILTRATION.: 08/06/1999. }\end{array}$ & LB990861233129.001 \\
\hline Sub-flowchart 11 11 & $\begin{array}{l}\text { DRIFT SCALE CALIBRATED 1-D PROPERTY SET, } \\
\text { FY99: LOWER BOUND INFILTRATION.: 08/06/1999. }\end{array}$ & LB990861233129.003 \\
\hline DATA STORE I & $\begin{array}{l}\text { DRIFT SCALE CALIBRATED 1-D PROPERTY SET, } \\
\text { FY99: UPPER BOUND INFILTRATION.: 08/06/1999. }\end{array}$ & LB990861233129.002 \\
\hline Sub-flowchart 11 & $\begin{array}{l}\text { THERMAL PROPERTIES AND TORTUOSITY FACTOR } \\
\text { FOR THE UZ MODEL LAYERS FOR AMR U0090, } \\
\text { "ANALYSIS OF HYDROLOGIC PROPERTIES DATA." } \\
\text { Submittal Date: 15-OCt-1999. }\end{array}$ & LB991091233129.006 \\
\hline DATA STORE J flowchart 16
\end{tabular}


Table 3 Software and routines used and the software tracking numbers (STN).

\begin{tabular}{|c|c|c|c|c|}
\hline Code & STN & Software Type & Section & $\begin{array}{c}\text { Sub- } \\
\text { flowchart }\end{array}$ \\
\hline NUFT v3.0s & $10088-3.0$ s-00 & Software & 5.1 .1 & $13,14,15,16$ \\
\hline RADPRO V3.22 & $10204-3.22-00$ & Software & 5.1 .2 & 15 \\
\hline XTOOL v10.1 & $10208-10.1-00$ & Software & 5.1 .3 & N/A \\
\hline MSTHAC V6.4.3 & $10419-6.4 .3-00$ & Software & 5.1 .4 & $\mathrm{~N} / \mathrm{A}$ \\
\hline CONVERTCOORDS V1.1 & $10209-1.1-00$ & Routine & 5.2 .1 & 8 \\
\hline YMESH V1.53 & $10172-1.53-00$ & Routine & 5.2 .2 & $1,2,3,4$ \\
\hline makeColumns v1.1 & Currently under $\mathrm{Q} / \mathrm{A}$ & Routine & 5.2 .3 & 2,3 \\
\hline define_EBS_fineGrid v1.4 & Currently under $Q / A$ & Routine & 5.2 .4 & 2 \\
\hline reads Units v1.0 & $10602-1.0-00$ & Routine & 5.2 .5 & 2 \\
\hline addLayers $v 1.0$ & $10603-1.0-00$ & Routine & 5.2 .6 & 3 \\
\hline addlay $v 1.0$ & $10604-1.0-00$ & Routine & 5.2 .7 & 7 \\
\hline infiltab v1.0 & $10608-1.0-00$ & Macro & 5.2 .10 & 8 \\
\hline rock_LDTH v.10 & $10 ? ? ?-1.0-00$ & Macro & 5.2 .11 & 11 \\
\hline rock_SDT V1.0 & $10 ? ? ?-1.0-00$ & Macro & 5.2 .12 & 11 \\
\hline SMT_surf_bc3 v1.1 & Currently under $\mathrm{Q} / \mathrm{A}$ & Routine & 5.2 .13 & 9 \\
\hline SMT_bot_bc3 v1.1 & Currently under $\mathrm{Q} / \mathrm{A}$ & Routine & 5.2 .14 & 9 \\
\hline bound v1.0 & $10611-1.0-00$ & Macro & 5.2 .15 & 9 \\
\hline heatgenAge v1.2 & $10612-1.2-00$ & Routine & 5.2 .16 & $5,6,7$ \\
\hline chim_surf_wt_TP2 v1.0 & Currently under $\mathrm{Q} / \mathrm{A}$ & Routine & 5.2 .9 & 9 \\
\hline columninfiltration v1.2 & Currently under $\mathrm{Q} / \mathrm{A}$ & Routine & 5.2 .8 & 8 \\
\hline StudyDomain v1.0 & Currently under $\mathrm{Q} / \mathrm{A}$ & Macro & 5.2 .18 & 2,3 \\
\hline rme6 v1.1 & $10617-1.1-00$ & Routine & 5.2 .17 & $1,2,3,4$ \\
\hline LDTH-01-li-i & Currently under $\mathrm{Q} / \mathrm{A}$ & Routine & 5.2 .19 & 10,13 \\
\hline LDTH-01-mi-i & Currently under $\mathrm{Q} / \mathrm{A}$ & Routine & 5.2 .19 & 10,13 \\
\hline LDTH-01-ui-i & Currently under $\mathrm{Q} / \mathrm{A}$ & Routine & 5.2 .19 & 10,13 \\
\hline LDTH-01-i-v & Currently under $\mathrm{Q} / \mathrm{A}$ & Routine & 5.2 .19 & 10,13 \\
\hline LDTH-01-mi-V & Currently under $\mathrm{Q} / \mathrm{A}$ & Routine & 5.2 .19 & 10,13 \\
\hline LDTH-01-ui-v & Currently under $Q / A$ & Routine & 5.2 .19 & 10,13 \\
\hline LDTH-01-1i & Currently under $\mathrm{Q} / \mathrm{A}$ & Routine & 5.2 .19 & 10,13 \\
\hline LDTH-01-mi & Currently under $\mathrm{Q} / \mathrm{A}$ & Routine & 5.2 .19 & 10,13 \\
\hline LDTH-01-ui & Currently under $\mathrm{Q} / \mathrm{A}$ & Poutine & 5.2 .19 & 10,13 \\
\hline LDTH-03-li-v & Currently under $\mathrm{Q} / \mathrm{A}$ & Routine & 5.2 .19 & 10,13 \\
\hline LDTH-03-mi-v & Currently under $\mathrm{Q} / \mathrm{A}$ & Routine & 5.2 .19 & 10,13 \\
\hline LDTH-03-ui-v & Currently under $\mathrm{Q} / \mathrm{A}$ & Routine & 5.2 .19 & 10,13 \\
\hline LDTH-03-1i & Currently under $\mathrm{Q} / \mathrm{A}$ & Routine & 5.2 .19 & 10,13 \\
\hline LDTH-03-mi & Currently under $\mathrm{Q} / \mathrm{A}$ & Routine & 5.2 .19 & 10,13 \\
\hline LDTH-03-ui & Currently under $\mathrm{Q} / \mathrm{A}$ & Routine & 5.2 .19 & 10,13 \\
\hline SDT-01i & $10628-1.0-00$ & Routine & 5.2 .19 & 13 \\
\hline SDT-01 & $10629-1.0-00$ & Routine & 5.2 .19 & 13 \\
\hline SDT-01V & $10630-1.0-00$ & Routine & 5.2 .19 & 13 \\
\hline SDT-03 & Currently under $\mathrm{Q} / \mathrm{A}$ & Routine & 5.2 .19 & 13 \\
\hline SDT-03v & Currently under $\mathrm{Q} / \mathrm{A}$ & Routine & 5.2 .19 & 13 \\
\hline
\end{tabular}


Table 4 N-table for data flow.

\begin{tabular}{|c|c|c|c|}
\hline N:M1 & SF-3,4 & SDT:Lcolumn_template & Mesh column_template for LDTH and SDT \\
\hline $\mathrm{N}: \mathrm{M} 2$ & SF-5 & LDTH:Icolumn_template & $\begin{array}{l}\text { The grid files for the SMT model are specified by applying several criteria: } \\
\text { - The east boundary is to be parallel to the actual repository boundary. } \\
\text { - The repository edge has a finer grid to allow the MSTHM algorithm to treat edge } \\
\text { effects appropriately. } \\
\text { - The grid blocks are each to correspond to an integral number of dritts in order to } \\
\text { simplify the final parameter mapping. }\end{array}$ \\
\hline N:L1 & SF-13 & $\begin{array}{l}\text { run_control_param_LDTH-v00 } \\
\text { run_control_param_LDTH-v01 }\end{array}$ & $\begin{array}{l}\text { Run control parameters provide setup of the numerical control of the simulation } \\
\text { code itself to achieve reasonable efficiency in the computation }\end{array}$ \\
\hline N:L2 & SF -13 & $\begin{array}{l}<\text { aml }>\text { infil } \\
<\text { aml }>\text { pre-closure } \\
<\text { <aml }>\text { post-closure }\end{array}$ & LDTH Templates \\
\hline N:L3 & SF-13 & $\begin{array}{l}\text { output.times-00v } \\
\text { output.times-<aml }>-00\end{array}$ & $\begin{array}{l}\text { Run control parameters provide selup of the numerical control of the simulation } \\
\text { code itself to achieve reasonable efficiency in the computation }\end{array}$ \\
\hline $\mathrm{N}: \mathrm{S1}$ & SF.14 & SDT-00tplt & SDT Templates \\
\hline $\mathrm{N}: \mathrm{S} 2$ & SF-14 & $\begin{array}{l}\text { output.times-00v } \\
\text { output.times-<ami>-20 }\end{array}$ & $\begin{array}{l}\text { The output times files contain the list of times for which parameter values are to be } \\
\text { written to the output stream. The criterion for the choice of times is to achieve } \\
\text { suitable time resolution, especially when the relative humidity in the drift is varying } \\
\text { rapidly. An appropriate choice of times was discovered empirically }\end{array}$ \\
\hline N:D1 & SF-15 & $\begin{array}{l}\text { run_control_param_DDT-v01 } \\
\text { run_control_param_DDT-v02 }\end{array}$ & $\begin{array}{l}\text { Run control paramelers provide setup of the numerical control of the simulation } \\
\text { code itself to achieve reasonable efficiency in the computation }\end{array}$ \\
\hline N:D2 & SF-15 & output times-DOT60-20 & $\begin{array}{l}\text { The output times files contain the list of times for which parameter values are to be } \\
\text { written to the output stream. The criterion for the choice of times is to achieve } \\
\text { suitable time resolution, especially when the relative humidity in the drift is varying } \\
\text { rapidly. An appropriate choice of times was discovered empirically }\end{array}$ \\
\hline N:D3 & SF-15 & $\begin{array}{l}\text { DDT60-03.radin } \\
\text { DDT60-03v.radin } \\
\text { DDT60-03pbt.radin }\end{array}$ & $\begin{array}{l}\text { The radin files are used to specify which surfaces may be coupled via radiation, and } \\
\text { to provide emissivity assignments to the surfaces. }\end{array}$ \\
\hline $\mathrm{N}: \mathrm{T} 1$ & SF-16 & run_control_param_SMT-v01 & $\begin{array}{l}\text { Run control paramelers provide setup of the numerical control of the simulation } \\
\text { code itself to achieve reasonable etficiency in the computation. }\end{array}$ \\
\hline N:T2 & SF-16 & output.times-SMT56-20 & $\begin{array}{l}\text { The output times files contain the list of times for which parameter values are to be } \\
\text { written to the output stream. The criterion for the choice of times is to achieve } \\
\text { suitable time resolution, especially when the relative humidity in the dritt is varying } \\
\text { rapidly. An appropriate choice of times was discovered empirically }\end{array}$ \\
\hline N:T3 & SF-16 & SMT-1Dds-mi-sz & $\begin{array}{l}\text { Set properties of natural system materials in the saturated zone. The material is } \\
\text { assumed to be identical to that found at the bottom of the unsaturated zone. }\end{array}$ \\
\hline
\end{tabular}

- This column gives the alpha numeric designator used in the figure referenced in the "Fig." column.

N:xx allows a free-form textual note to be attached to a given operation. In this case, there is not typically an input file to the step, but the information is originated in some sort of analyst input from domain expertise, and is at this point encapsulated. 
Table 5 E-table for data flow.

\begin{tabular}{|c|c|c|c|c|}
\hline$\alpha \#$ & Fig. & Input File Name(s) & Output File Names ${ }^{* \hbar}$ & Notes \\
\hline E:M1 & SF-1 & |15c3.dat & & $\begin{array}{l}\text { Refine the input LDTH grid to sufficiently describe therma } \\
\text { radiation processes. }\end{array}$ \\
\hline $\mathrm{E}: \mathrm{M} 2$ & SF-1 & Data Store B, 15c3.03v.dat & $15 c 3$. dat & $\begin{array}{l}\text { Reline grid within drift to properly describe post-closure } \\
\text { engineered system material distribution, especially drip shield } \\
\text { emplacement. }\end{array}$ \\
\hline $\mathrm{E}: \mathrm{M3}$ & $\begin{array}{l}\text { SF-2 } \\
\text { SF-3 }\end{array}$ & shape.dat & column.data & $\begin{array}{l}\text { Chimney locations are chosen in a regular array parallel to the } \\
\text { major axis (East side) of the MSTHM repository footprint. }\end{array}$ \\
\hline $\begin{array}{l}E: H 1 \\
E: H 2\end{array}$ & $\begin{array}{l}\text { SF-5 } \\
\text { SF-7 }\end{array}$ & $\begin{array}{l}\text { Waste_Package_Design_Informat_1.doc } \\
\text { DDT-heats-2.xls }\end{array}$ & $\begin{array}{l}\text { SMT-heatgen-truncated } \\
\text { DDT-heatgen-01 }\end{array}$ & $\begin{array}{l}\text { Removed the column headers and reorganized the parameter } \\
\text { values into a matrix format for Matlab to read. }\end{array}$ \\
\hline $\mathrm{E}: \mathrm{H3}$ & SF-6 & Waste_Package_Design_Informat_1.doc & $\begin{array}{l}\text { line-load-heat-1.13 } \\
\text { line-load-heat-1.35 }\end{array}$ & $\begin{array}{l}\text { Place the data columns of Time and Averaged Heat Transfer } \\
\text { into NUFT format (in a compllux block). }\end{array}$ \\
\hline$E: B 1$ & SF-9 & surface_TP-RH.out & $\begin{array}{l}\text { temperature_w } \\
\text { temperature_s }\end{array}$ & $\begin{array}{l}\text { The files chimney_wt_TP and chimney_surface_TP are } \\
\text { transformed into temperature_W and temperature_S, } \\
\text { respectively, by laying out the temperature data found in the } \\
\text { source file into an array format of } 7 \times 5 \text { rather than a list. All } \\
\text { other (non-temperature) source data is discarded. }\end{array}$ \\
\hline $\begin{array}{l}E: C 1 \\
E: C 1 \\
E: C 1\end{array}$ & $\begin{array}{l}\text { SF-10 } \\
\text { SF-10 } \\
\text { SF-10 }\end{array}$ & $\begin{array}{l}B-1, B-2,1-L 0 \\
B-1, B-2,1-M O \\
B-1, B-2, I-U 0\end{array}$ & $\begin{array}{l}\text { LDTH-01-li-I } \\
\text { LDTH-01-mi-I } \\
\text { LDTH-01-ui-I }\end{array}$ & LDTH initial script \\
\hline $\begin{array}{l}E: C 2 \\
E: C 2 \\
E: C 2\end{array}$ & $\begin{array}{l}\text { SF-10 } \\
\text { SF-10 } \\
\text { SF-10 }\end{array}$ & $\begin{array}{l}\text { B-1, B-2, I-LO, I-L1 } \\
\text { B-1, B-2, I-MO, I-M1 } \\
\text { B-1, B-2, I-UO, I-U1 }\end{array}$ & $\begin{array}{l}\text { LDTH-0X-li-v } \\
\text { LDTH-0X-mi-v } \\
\text { LDTH-0X-ui-v }\end{array}$ & LDTH ventilation script \\
\hline $\begin{array}{l}E: C 3 \\
E: C 3 \\
E: C 3\end{array}$ & $\begin{array}{l}\text { SF-10 } \\
\text { SF-10 } \\
\text { SF-10 }\end{array}$ & $\begin{array}{l}\text { B-1, B-2, I-LO, I-L1 } \\
\text { B-1, B-2, I-M0, I-M1 } \\
\text { B-1, B-2, I-U0, I-U1 }\end{array}$ & $\begin{array}{l}\text { LDTH-OX-li } \\
\text { LDTH-OX-mi } \\
\text { LDTH-OX-ui }\end{array}$ & LDTH main script \\
\hline E:R1 & SF-11 & dkm-afc-1Dds-mc-mi-00 & SDT-1Dds-mi & $\begin{array}{l}\text { Removed all hydrologic properties and set thermal properties to } \\
\text { the appropriate wet material properties. }\end{array}$ \\
\hline E:P1 & SF-12 & DTN unknown file name & $R-1, R-1 v, R-2, R-3, R-D$ & $\begin{array}{l}\text { Tabulated In-dritt and Thermal Properties Used in Drift-scale } \\
\text { Models lor TSPA-SR (Rev 01) to create EBS material } \\
\text { properties files }\end{array}$ \\
\hline E:S1 & SF-14 & $\overline{B-1}$ & SDT-OXi & SDT initial script \\
\hline E:S2 & SF-14 & $B-1$ & SDT-OXV & SDT ventilation script \\
\hline E:S3 & SF-14 & B-1 & SDT-0X & SDT main script \\
\hline E:D1 & SF-15 & M-D1 & $|5 c 3-D D T<a m|>-0 X v$.in & DDT initial script \\
\hline $\mathrm{E}: \mathrm{D} 2$ & SF-15 & M-D2 & 15c3-DDT<aml>-0Xv.in & DOT ventilation script \\
\hline E:D3 & SF-15 & M-D2 & |5c3-DDT<am $\mid>-0 X$. in & DDT main script \\
\hline $\mathrm{E}: \mathrm{T} 1$ & SF-16 & thermal_UZ.xls & SMT-1Dds-mi-flt & $\begin{array}{l}\text { Manually format rock property information for materials in fault } \\
\text { areas into NUFT input file format. }\end{array}$ \\
\hline
\end{tabular}


Table 6 File groups for data flow.

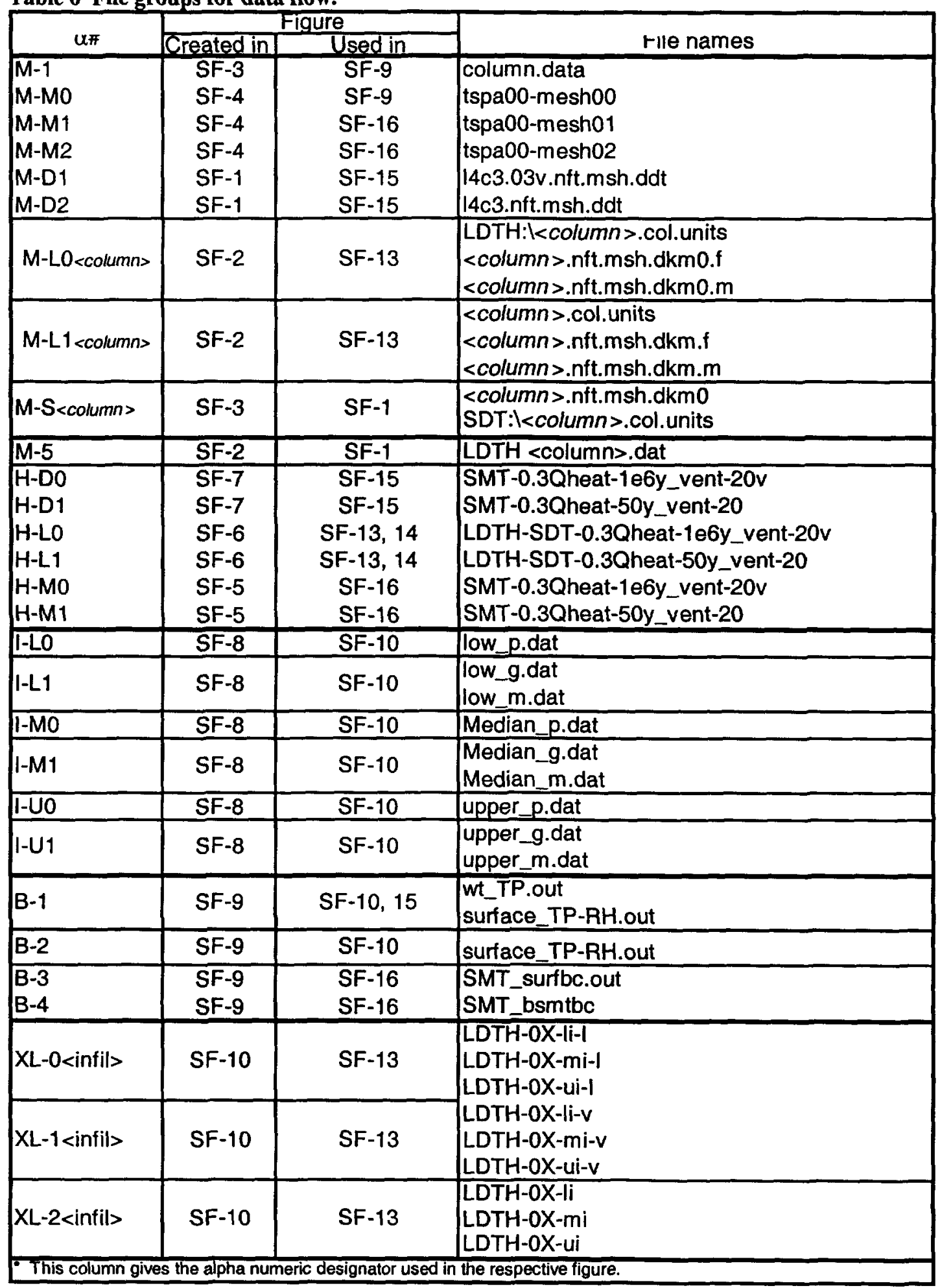


Table 6 (cont'd). File groups for data flow

\begin{tabular}{|c|c|c|c|}
\hline$\alpha \#$ & Origin & Dest. & File names \\
\hline R-S & SF-11 & SF-14, 15, 16 & SDT-1Dds \\
\hline$R-L<$ infil $>$ & SF-11 & SF-13 & $\begin{array}{l}\text { dkm-afc-1Dds-mc-li-01 } \\
\text { dkm-afc-1Dds-mc-mi-01 } \\
\text { dkm-afc-1Dds-mc-ui-01 }\end{array}$ \\
\hline $\begin{array}{l}R-1 \\
R-1 v \\
R-3 \\
R-D\end{array}$ & $\begin{array}{l}\text { SF-12 } \\
\text { SF-12 } \\
\text { SF-12 } \\
\text { SF-12 }\end{array}$ & $\begin{array}{l}\text { SF-13, } 15 \\
\text { SF-13 } \\
\text { SF-13 } \\
\text { SF-15 }\end{array}$ & $\begin{array}{l}\text { modprop100_0X_<aml> } \\
\text { modprop_dr-0Xv } \\
\text { dkm-afc-EBS_Rev100-0X } \\
\text { DDT-EBS_Rev100 }\end{array}$ \\
\hline $\begin{array}{l}\text { LDTH }-0<A I C> \\
\text { LDTH-1<AIC> }\end{array}$ & $\begin{array}{l}\text { SF-13 } \\
\text { SF-13 }\end{array}$ & $\begin{array}{l}\text { LDTH out } \\
\text { LDTH out }\end{array}$ & $\begin{array}{l}<\text { column>-LDTH<aml>-1Dds_mc-<infil>-0X.m.EBS.ext } \\
<\text { column>-LDTH<aml>-1Dds_mc-<infil>-0X.f.EBS.ext }\end{array}$ \\
\hline $\begin{array}{l}\text { SDT-0<AC> } \\
\text { SDT- } 1<A C>\end{array}$ & $\begin{array}{l}\mathrm{SF}-14 \\
\mathrm{SF}-14 \\
\end{array}$ & $\begin{array}{l}\text { SDT out } \\
\text { SDT out }\end{array}$ & $\begin{array}{l}<\text { column }>-S D T<\text { aml }>-0 X v . \text { ext } \\
\text { <column }>- \text { SDT<aml }>-0 X . e x t\end{array}$ \\
\hline $\begin{array}{l}S-Z \\
\text { DDT-0 } \\
\text { DDT-1 }\end{array}$ & $\begin{array}{l}\text { SF-14 } \\
\text { SF-15 } \\
\text { SF-15 }\end{array}$ & $\begin{array}{l}\text { SF-15 } \\
\text { DDT out } \\
\text { DDT out }\end{array}$ & $\begin{array}{l}15 c 3-S D T-01-i . z t a b \\
15 c 3-D D T<a m 1>-0 X v . E B S . e x t \\
15 c 3-D D T<a m 1>-0 X . E B S . e x t\end{array}$ \\
\hline $\begin{array}{l}\text { SMT-0 } \\
\text { SMT-1 }\end{array}$ & $\begin{array}{l}\text { SF-16 } \\
\text { SF-16 }\end{array}$ & $\begin{array}{l}\text { SMT out } \\
\text { SMT out }\end{array}$ & $\begin{array}{l}\text { SMT<aml>-0Xv.ext } \\
\text { SMT<aml>-OX.ext }\end{array}$ \\
\hline
\end{tabular}

* This column gives the alpha numeric designator used in the respective figure. 
Table 7 Software routine input and output files.

\begin{tabular}{|c|c|c|c|c|c|c|}
\hline Name/Number & $\begin{array}{l}\text { Data } \\
\text { Flowchart } \\
\text { Figure }\end{array}$ & Input source & Input File name & \# of files & Output File Name & $\begin{array}{l}\text { \# of } \\
\text { files }\end{array}$ \\
\hline \multirow{2}{*}{ addlayers v1.0 } & SF.3 & YMESH output & $15 c 3 . n f t$ & 1 & 15c3.nft.msh.ddt & 1 \\
\hline & SF-1 & YMESH output & 15c3.03v.nft & 1 & 15c3.03v.nft.msh.ddt & 1 \\
\hline bound.m v1.0 & SF-9 & DATA STORE A & tcond_wet.dat & 1 & SMT_basement_temp & 1 \\
\hline 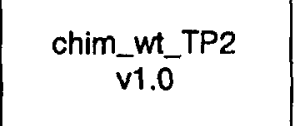 & SF.9 & $\begin{array}{l}\text { DATA STORE A } \\
\text { DATA STORE F }\end{array}$ & $\begin{array}{c}\text { tspa99_primary_mesh } \\
\text { bcs_99.dat }\end{array}$ & $\begin{array}{l}1 \\
1\end{array}$ & $\begin{array}{l}\text { wt_PT.out } \\
\text { surface_TP-RH.out } \\
\text { surf_TP.out }\end{array}$ & 3 \\
\hline $\begin{array}{c}\text { CONVERTCOORDS } \\
\vee 1.1\end{array}$ & SF-8 & DATA STORE E & *.dat & 9 & ${ }^{\star} . N V$ & 9 \\
\hline Cover v1.1 & SF-2 & DATA STORE C & dft1.dat & 1 & $\begin{array}{l}\text { shape1.dat } \\
\text { figure1.dat }\end{array}$ & 1 \\
\hline $\begin{array}{c}\text { define_EBS_fine } \\
\text { Grid v1.4 }\end{array}$ & $\mathrm{SF} \cdot 2$ & YMESH output & LDTH: $<$ column>.nft & 33 & $\begin{array}{l}\text { <column>.nft.msh.dkm.f } \\
\text { <column>.nft.msh.dkm.m } \\
\text { <column>.nft.msh.dkm0.f } \\
\text { <column>.nft.msh.dkm0.m }\end{array}$ & $\begin{array}{l}33 \\
33 \\
33 \\
33\end{array}$ \\
\hline
\end{tabular}


Table 7 (cont'd). Software routine input and output files.

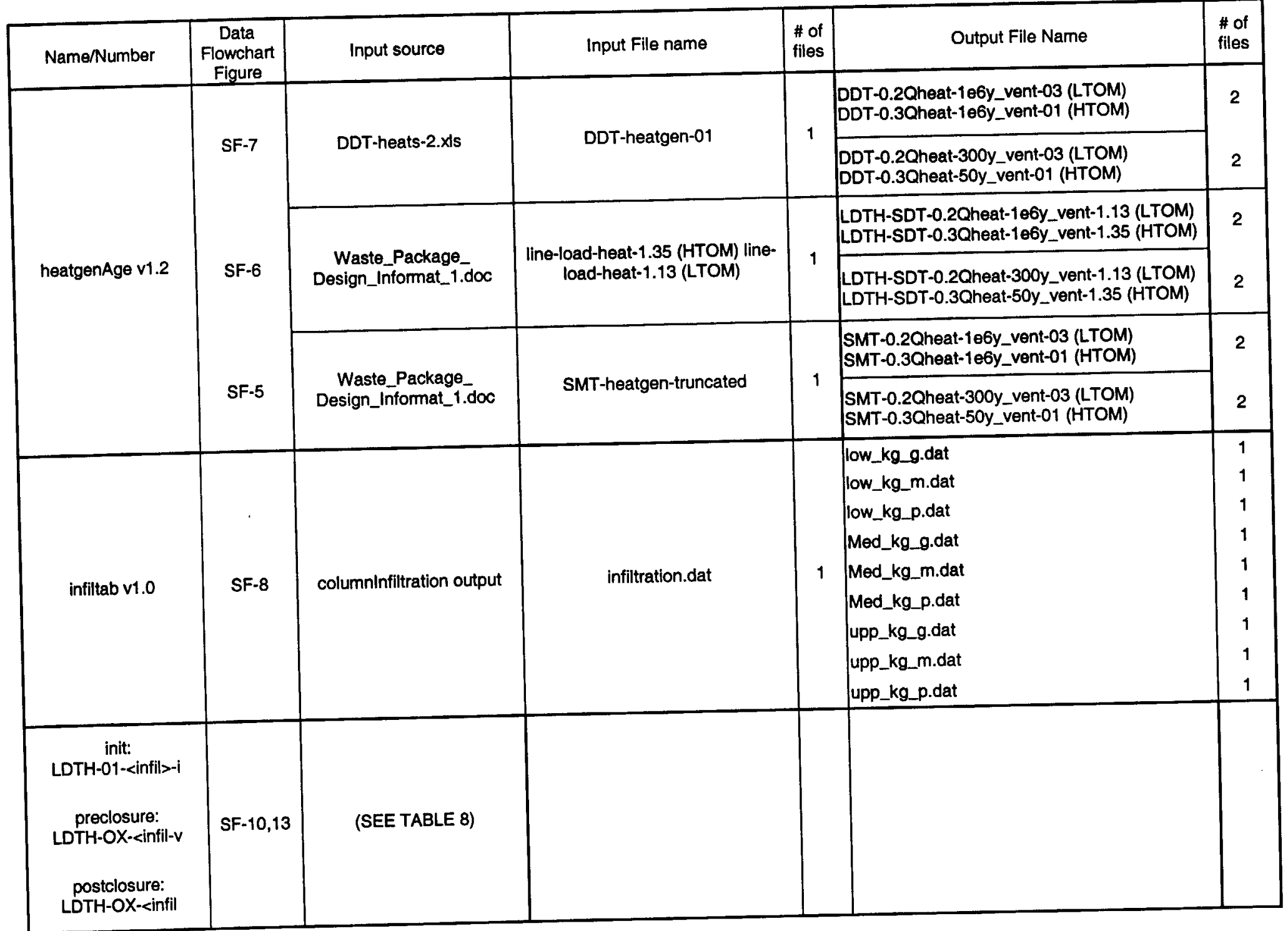


Table 7 (cont'd). Software routine input and output files.

\begin{tabular}{|c|c|c|c|c|c|c|}
\hline Name/Number & $\begin{array}{l}\text { Data } \\
\text { Flowchart } \\
\text { Figure }\end{array}$ & Input source & Input File name & $\begin{array}{l}\text { \# of } \\
\text { files }\end{array}$ & Output File Name & $\begin{array}{l}\text { \# of } \\
\text { files }\end{array}$ \\
\hline \multirow{2}{*}{$\begin{array}{c}\text { makeColumn_2000 } \\
\text { v1.0 }\end{array}$} & SF-2 & $\begin{array}{l}\text { N:M1 } \\
\text { E:M3 }\end{array}$ & $\begin{array}{l}\text { LDTH:Icolumn_template } \\
\text { column.data }\end{array}$ & $\begin{array}{l}1 \\
1\end{array}$ & LDTH:kcolumn>.dat & 33 \\
\hline & SF-3 & $\begin{array}{l}N: M 1 \\
E: M 3\end{array}$ & $\begin{array}{l}\text { SDT: lcolumn_template } \\
\text { column.data }\end{array}$ & $\begin{array}{l}1 \\
1 \\
\end{array}$ & SDT:kcolumns, dat & 33 \\
\hline \multirow{2}{*}{ readsUnits v1.0 } & \multirow{2}{*}{ SF-2,3 } & YMESH output & LDTH:kcolumn>.col & 33 & LDTH: $<$ column>.col.units & 33 \\
\hline & & YMESH output & SDT:kcolumn>.col & 33 & SDT:kcolumn>.col.units & 33 \\
\hline rme6 v1.1 & $\begin{array}{l}\text { SF-1 } \\
\text { SF-2 } \\
\text { SF-3 } \\
\text { SF-4 }\end{array}$ & DATA STORE A & tspa_primary_mesh & 1 & LBL99-YMESH & 1 \\
\hline \multirow{4}{*}{$\begin{array}{c}\text { rock_LDTH v1.0 and } \\
\text { rock_SDT v } 1.0\end{array}$} & \multirow{4}{*}{ SF-11 } & $\frac{\text { DATA STORE G }}{\text { DATA STORE G }}$ & $\begin{array}{l}\text { 1d_driftscale_basecase2_flow.pm } \\
\text { 1d_driftscale_basecase2_th.pm }\end{array}$ & $\begin{array}{l}1 \\
1\end{array}$ & dkm-afc-1Dds-mc-mi-01 & 1 \\
\hline & & $\begin{array}{l}\text { DATA STORE I } \\
\text { DATA STORE I }\end{array}$ & $\begin{array}{l}\text { 1d_driftscale_lowinf_flow.prn } \\
\text { 1d_driftscale_lowinf_th.pm }\end{array}$ & $\begin{array}{l}1 \\
1 \\
\end{array}$ & dkm-afc-1Dds-mc-li-01 & 1 \\
\hline & & DATA STORE H & & 1 & dkm-afc-1Dds-mc-ui-01 & 1 \\
\hline & & DATA STORE H & 1d_driftscale_upperinf_th.pm & 1 & & \\
\hline & & $\begin{array}{l}\text { E:S1 } \\
N: S 1\end{array}$ & $\begin{array}{c}\text { SDT-01i } \\
\text { SDT-c01-I-tplt }\end{array}$ & $\begin{array}{l}1 \\
1\end{array}$ & SDT initial. $<$ column $><a m l>$ & 1 \\
\hline $\begin{array}{c}\text { scripts: SDT-01i, } \\
\text { SDT-0Xv, SDT-0X* } \\
\text { v1.0 }\end{array}$ & SF-14 & $\begin{array}{l}\mathrm{E}: \mathrm{S} 2 \\
\mathrm{~N}: \mathrm{S} 1\end{array}$ & $\begin{array}{c}\text { SDT-0XV } \\
\text { SDT-c01-I-tplt } \\
\end{array}$ & $\begin{array}{l}1 \\
1 \\
\end{array}$ & $<$ column $>-$ SDT $<$ aml $>-20$.in & 1 \\
\hline & & $\begin{array}{l}\text { E:S3 } \\
\text { N:S1 }\end{array}$ & $\begin{array}{c}\text { SDT-0X } \\
\text { SDT-C01-tplt }\end{array}$ & $\begin{array}{l}1 \\
1\end{array}$ & $<$ column>-SDT<aml>-20v.in & 1 \\
\hline SMT_surt_bc3 v1.0 & SF_9 & chim_surf_wt_TP2 output & surf_TP.out & 1 & $\begin{array}{c}\text { SMT_surfbc.out (name chosen } \\
\text { by user) }\end{array}$ & 1 \\
\hline StudyDomain v1.0 & SF-2,3 & DATA STORE C & E:M4 & 1 & footprint.dat & 1 \\
\hline
\end{tabular}


Table 7 (cont'd). Software routine input and output files.

\begin{tabular}{|c|c|c|c|c|c|c|}
\hline Name/Number & $\begin{array}{l}\text { Data } \\
\text { Flowchart } \\
\text { Figure }\end{array}$ & Input source & Input File name & $\begin{array}{l}\text { \# of } \\
\text { files }\end{array}$ & Output File Name & $\begin{array}{l}\text { \# of } \\
\text { files }\end{array}$ \\
\hline SMT_bot_bc3 v1.0 & SF.9 & $\begin{array}{l}\text { bound output } \\
\text { YMESH output }\end{array}$ & $\begin{array}{l}\text { SMT_basement_temp } \\
\text { tspa01.meshoX-03 }\end{array}$ & $\begin{array}{l}1 \\
1\end{array}$ & $\begin{array}{c}\text { SMT_bsmtbc (chosen by } \\
\text { user) }\end{array}$ & 1 \\
\hline YMESH V1.53 & SF 1 & DATA STORE A, B & $\begin{array}{c}\text { LBL99-YMESH, 15c3.dat, } \\
\text { 15c3.03v.dat }\end{array}$ & & $15 c 3 . n f t, 15 c 3.03 \mathrm{v} . n f t$ & \\
\hline YMESH v1.53 & SF 2 & DATA STORE A, C & $\begin{array}{c}\text { LBL99-YMESH, } \\
\text { <column>.dat from LDTH }\end{array}$ & & $\begin{array}{l}<\text { column>.nft for LDTH, } \\
<\text { column>.col for LDTH }\end{array}$ & \\
\hline YMESH v1.53 & SF 3 & DATA STORE A, C & $\begin{array}{l}\text { LBL99-YMESH, } \\
\text { <column>.dat from SDT }\end{array}$ & & $\begin{array}{l}\text { <column>.nft for SDT, } \\
\text { <column>.col for SDT }\end{array}$ & \\
\hline YMESH v1.53 & SF 4 & DATA STORE A, N:M2 & $\begin{array}{l}\text { LBL99-YMESH, } \\
\text { tspa01.grid01-03, } \\
\text { tspa01.grid03-03 }\end{array}$ & & $\begin{array}{l}\text { tspa01.mesh01-03, } \\
\text { tspa01.mesh03-03 }\end{array}$ & \\
\hline
\end{tabular}


Table 8 LDTH NUFT input and output files

\begin{tabular}{|c|c|c|c|c|c|c|}
\hline Description & $\begin{array}{c}\text { Data } \\
\text { Flowchart } \\
\text { Figure }\end{array}$ & Input source & File $\alpha \#$ & Input File name & $\begin{array}{l}\text { \# of } \\
\text { files }\end{array}$ & Output File Name \\
\hline \multirow{12}{*}{$\begin{array}{l}\text { LDTH assembly } \\
\text { script for } \\
\text { initialization: } \\
\text { LDTH-01-<infil>-i }\end{array}$} & \multirow{12}{*}{ SF $-10,13$} & \multirow{2}{*}{ define_EBS_fineGrid output } & \multirow{3}{*}{$\begin{array}{c}\text { M-LO } \\
<\text { column> }\end{array}$} & $<$ column>.nft.msh.dkmo.f & 33 & \multirow{12}{*}{$<$ column $>-$ LDTH $<$ aml $>-1$ Dds_mc- $<$ infil $>-$ i. in } \\
\hline & & & & $<$ column>.nft.msh.dkm0.m & 33 & \\
\hline & & readUnits output & & LDTH:kcolumn>.col.units & 33 & \\
\hline & & & & dkm-afc-1Dds-mc-mi-01 & 1 & \\
\hline & & rock_LDTH output & $R-L<$ infil> & dkm-afc-1Dds-mc-li-01 & 1 & \\
\hline & & & & dkm-afc-1Dds-mc-ui-01 & 1 & \\
\hline & & $\mathrm{N}: \mathrm{L} 1$ & & run_control_param_LDTH-v09 & 1 & \\
\hline & & \multirow{3}{*}{ columnInfiltration output } & I-UO & upp_kg_p.dat & 1 & \\
\hline & & & I-MO & Med_kg_p.dat & 1 & \\
\hline & & & I-LO & low_kg_p.dat & 1 & \\
\hline & & chim_surf_Wt_TP2 output & B-1 & $\begin{array}{c}\text { wt_TP.out } \\
\text { surface_TP-RH.out }\end{array}$ & 2 & \\
\hline & & template & & LDTH<aml>-c00-i-tplt (template) & 1 & \\
\hline
\end{tabular}


Table 8 LDTH NUFT input and output files (cont)

\begin{tabular}{|c|c|c|c|c|}
\hline File $\alpha \#$ & Input File name & $\begin{array}{l}\text { \# of } \\
\text { files }\end{array}$ & Output File Name & $\begin{array}{l}\text { \# of } \\
\text { files }\end{array}$ \\
\hline \multirow{3}{*}{$\begin{array}{c}\text { M-L1 } \\
\text { <column> }\end{array}$} & <column>.nft.msh.dkm.f & 33 & \multirow{19}{*}{$\begin{array}{c}<\text { column>-LDTH<aml>-1Dds_mc-<infil>- } \\
O X v \text {.in }\end{array}$} & \multirow{19}{*}{792} \\
\hline & $<$ column>.ntt.msh.dkm.m & 33 & & \\
\hline & LDTH:Kcolumn>.col.units & 33 & & \\
\hline \multirow{3}{*}{ R-L <infil> } & dkm-afc-1Dds-mc-mi-01 & 1 & & \\
\hline & dkm-afc-1Dds-mc-li-01 & 1 & & \\
\hline & $d k m-a f c-1 D d s-m c-u i-01$ & 1 & & \\
\hline R-1v & modprop_dr-0Xv & 1 & & \\
\hline B.1 & $\begin{array}{l}\text { wt_TP.out } \\
\text { surface_TP-RH.out }\end{array}$ & 2 & & \\
\hline \multirow[t]{4}{*}{ H-LO } & $\begin{array}{l}\text { LDTH-SDT-0.2Qheat-1e6y_vent-1.13 (LTOM) } \\
\text { LDTH-SDT-0.30heat-166y_vent- } 1.35 \text { (HTOM) }\end{array}$ & 2 & & \\
\hline & $\begin{array}{l}<c \text { column>-LDTH<aml>-1Dds_mc-<infill>-i.m.EBS.ext } \\
<\text { column>-LDTH<aml>-1Dds_mc-<infil>-i.f.EBS.ext }\end{array}$ & 792 & & \\
\hline & run_control_param_LDTH-VO9 & 1 & & \\
\hline & output.times_<amb-oX & 1 & & \\
\hline I-Uo & upp_kg_p.dat & 1 & & \\
\hline I-MO & Med_kg_p.dat & 1 & & \\
\hline 1.LO & low_kg_p.dat & 1 & & \\
\hline I-U1 & upp_kg_g.dat & 2 & & \\
\hline $1-M 1$ & Med_kg_g.dat & 2 & & \\
\hline \multirow[t]{2}{*}{ H-L1 } & $\begin{array}{l}\text { low_kg_g.dat } \\
\text { low kg m.dat }\end{array}$ & 2 & & \\
\hline & LDTH<aml>-c0x-v-tplt (template) & 1 & & \\
\hline
\end{tabular}


Table 8 LDTH NUFT input and output files (cont)

\begin{tabular}{|c|c|c|c|c|}
\hline File $\alpha \#$ & Input File name & $\begin{array}{l}\text { \# of } \\
\text { files }\end{array}$ & Output File Name & $\begin{array}{l}\text { \# of } \\
\text { files }\end{array}$ \\
\hline \multirow{3}{*}{$\begin{array}{l}\text { M-L1 } \\
<\text { column }>\end{array}$} & \multirow{2}{*}{$\begin{array}{l}<\text { column>.nft.msh.dkm.f } \\
<\text { column>.nft.msh.dkm.m }\end{array}$} & 33 & \multirow{20}{*}{ <column>-LDTH<aml>-1Dds_mc-<infil>-0X.in } & \multirow{20}{*}{792} \\
\hline & & 33 & & \\
\hline & LDTH: K<column>.col.units & 33 & & \\
\hline \multirow{3}{*}{$R-L<$ infils } & dkm-afc-1Dds-mc-mi-01 & 1 & & \\
\hline & dkm-afc-1Dds-mc-li-01 & 1 & & \\
\hline & dkm-afc-1Dds-mc-ui-01 & 1 & & \\
\hline R-3 & dkm-afc-EBS_Rev100-0X & 1 & & \\
\hline $\mathrm{R}-1$ & modprop_100_0X_<amls & 1 & & \\
\hline B-1 & $\begin{array}{l}\text { wt_TP.out } \\
\text { surface_TP-RH.out }\end{array}$ & 2 & & \\
\hline \multirow[t]{4}{*}{$H-L 1$} & $\begin{array}{l}\text { LDTH-SDT-0.2Qheat-300y_vent-1.13 (LTOM) } \\
\text { LDTH-SDT-0.30heat-50y_vent-1.35 (HTOM) }\end{array}$ & 2 & & \\
\hline & $\begin{array}{l}<\text { column>-LDTH<aml>-1Dds_mc-<infil>-0Xv.m.EBS.ext } \\
<\text { column>-LDTH<aml>-1Dds_mc-<infil>-0Xv.f.EBS.ext }\end{array}$ & 792 & & \\
\hline & run_control_param_LDTH-v09 & 1 & & \\
\hline & output.times_<aml>-OX & 1 & & \\
\hline I-UO & \multirow{6}{*}{$\begin{array}{l}\text { upp_kg_p.dat } \\
\text { Med_kg_p.dat } \\
\text { low_kg_p.dat } \\
\text { upp_kg_g.dat } \\
\text { upp_kg_m.dat } \\
\text { Med_kg_g.dat } \\
\text { Med_kg_m.dat } \\
\text { low_kg_g.dat } \\
\text { low_kg_m.dat }\end{array}$} & 1 & & \\
\hline I-MO & & 1 & & \\
\hline I-LO & & 1 & & \\
\hline I-U1 & & 2 & & \\
\hline I-M1 & & 2 & & \\
\hline \multirow[t]{2}{*}{ I-L1 } & & 2 & & \\
\hline & LDTH<aml>-cox-tplt (template) & 1 & & \\
\hline
\end{tabular}


Table 9 DDT NUFT input and output files.

\begin{tabular}{|c|c|c|c|c|c|c|c|}
\hline Description & $\begin{array}{l}\text { Data } \\
\text { Flowchart } \\
\text { Fioure }\end{array}$ & Input source & File $\alpha \#$ & Input File name & $\begin{array}{l}\# \text { of } \\
\text { files }\end{array}$ & Output File Name & $\begin{array}{l}\text { \# of } \\
\text { files }\end{array}$ \\
\hline \multirow[t]{2}{*}{$\begin{array}{l}\text { DDT } \\
\text { Initialization }\end{array}$} & \multirow[t]{2}{*}{ SF-15 } & $\begin{array}{c}\text { define_EBS_fineGrid output } \\
\text { readUnits output } \\
\text { E:P1 } \\
\text { NUFT output } \\
\text { heatgenAge output } \\
\text { N:D1 } \\
\text { N:D2 } \\
\text { Radpro output }\end{array}$ & $\begin{array}{c}R-1 \\
R-S \\
R-D \\
S-Z \\
\text { H-DO }\end{array}$ & $\begin{array}{c}\text { modprop_dr-20 } \\
\text { SDT-1Dds-mi } \\
\text { DDT-EBS_Rev20 } \\
\text { 14c3-SDT-01-I.ztab } \\
\text { DDT-0.3Qheat-1e6y_vent-00v } \\
\text { run_control_param_DDT-101 } \\
\text { output.times-DDT60-20 } \\
\text { DDT60-03v.radout } \\
\end{array}$ & $\begin{array}{l}1 \\
1 \\
1 \\
1 \\
1 \\
1 \\
1 \\
1 \\
\end{array}$ & \multicolumn{2}{|l|}{ Include files } \\
\hline & & E:D1 & M-D1 & 14c3-DDT60-20v.in & 1 & 14c3-DDT60-20v.EBS.ext & 1 \\
\hline \multirow[t]{2}{*}{$\begin{array}{l}\text { DDT } \\
\text { Preclosure }\end{array}$} & \multirow[t]{2}{*}{ SF-15 } & $\begin{array}{c}\text { E:P1 } \\
\text { readUnits output } \\
\text { E:P1 } \\
\text { NUFT output } \\
\text { heatgenAge output } \\
\text { N:D1 } \\
\text { N:D2 } \\
\text { Radpro output } \\
\end{array}$ & $\begin{array}{c}\text { R-1V } \\
\text { R-S } \\
\text { R-D } \\
\text { S-Z } \\
\text { H-D1 }\end{array}$ & $\begin{array}{c}\text { modprop_dr-20v } \\
\text { SDT-1Dds-mi } \\
\text { DDT-EBS_Rev20 } \\
\text { I4C3-SDT-01-1.ztab } \\
\text { DDT-0.3Qheat-50y_vent-00 } \\
\text { run_control_param_ODT-w01 } \\
\text { output.times-DDT60-20 } \\
\text { DDT60-03pbf.radout }\end{array}$ & $\begin{array}{l}1 \\
1 \\
1 \\
1 \\
1 \\
1 \\
1 \\
1\end{array}$ & Include files & \\
\hline & & E:D1 & M-D1 & 14c3-DDT60-20pbf.in & 1 & 14c3-DDT60-20pbt.res & 1 \\
\hline \multirow[t]{2}{*}{$\begin{array}{l}\text { DDT } \\
\text { Postclosure }\end{array}$} & \multirow[t]{2}{*}{ SF-15 } & $\begin{array}{c}\text { define_EBS_fineGrid output } \\
\text { readUnits output } \\
\text { E:P1 } \\
\text { NUFT output } \\
\text { heatgenAge output } \\
\text { N:D1 } \\
\text { N:D2 } \\
\text { Radpro output }\end{array}$ & $\begin{array}{l}\text { R-1 } \\
\text { R-S } \\
\text { R-D } \\
\text { H-D1 }\end{array}$ & $\begin{array}{c}\text { modprop_dr-20 } \\
\text { SDT-1Dds-mi } \\
\text { DDT-EBS_Rev20 } \\
\text { 14c3-DDT60-20pbf.res } \\
\text { DDT-0.3Qheat-50y_vent-00 } \\
\text { run_control_param_DDT-102 } \\
\text { output.times-DDT60-20 } \\
\text { DDT60-03pbf.radout } \\
\end{array}$ & $\begin{array}{l}1 \\
1 \\
1 \\
1 \\
1 \\
1 \\
1 \\
1 \\
\end{array}$ & \multicolumn{2}{|l|}{ Include files } \\
\hline & & E:D1 & M-D1 & 14c3-DDT60-20ss.in & 1 & 14c3-DDT60-20ss.EBS.ext & 1 \\
\hline
\end{tabular}


Table 10 SMT NUFT input and output.

\begin{tabular}{|c|c|c|c|c|c|c|c|}
\hline Description & $\begin{array}{l}\text { Data } \\
\text { Flowchart } \\
\text { Fiaure }\end{array}$ & Input source & File $\alpha \#$ & Input File name & $\begin{array}{l}\text { \# of } \\
\text { files }\end{array}$ & Output File Name & $\begin{array}{l}\text { \# of } \\
\text { files }\end{array}$ \\
\hline \multirow[t]{2}{*}{$\begin{array}{l}\text { SMT } \\
\text { Initialization }\end{array}$} & \multirow[t]{2}{*}{ SF-16 } & $\begin{array}{l}\text { readUnits output } \\
\text { heatgenAge output } \\
\text { YMESH output } \\
\text { N:T1 } \\
\text { N:T3 } \\
\text { E:T1 }\end{array}$ & $\begin{array}{c}\text { R-S } \\
\text { B-3 } \\
\text { M-M1 } \\
\text { B-4 }\end{array}$ & $\begin{array}{c}\text { SDT-1Dds-mi } \\
\text { SMT_surfbc } \\
\text { tspa00-mesh01 } \\
\text { run_control_param_SMT-v01 } \\
\text { SMT_bsmtbc } \\
\text { SMT-1Dds-mi-sz } \\
\text { SMT-1Dds-mi-flt }\end{array}$ & $\begin{array}{l}1 \\
1 \\
1 \\
1 \\
1 \\
1 \\
1\end{array}$ & \multicolumn{2}{|l|}{ Include files } \\
\hline & & user developed & & SMT60-01-i-in & 1 & SMT60-01-i-rst & 1 \\
\hline \multirow[t]{2}{*}{$\begin{array}{l}\text { SMT } \\
\text { Preclosure }\end{array}$} & \multirow[t]{2}{*}{ SF-16 } & $\begin{array}{l}\text { readUnits output } \\
\text { heatgenAge output } \\
\text { YMESH output } \\
\text { N:T1 } \\
\text { N:T2 } \\
\text { N:T3 } \\
\text { E:T1 } \\
\text { NUFT output }\end{array}$ & $\begin{array}{c}R-S \\
H-M O \\
M-M 2\end{array}$ & $\begin{array}{c}\text { SDT-1Dds-mi } \\
\text { SMT-0.3Qheat-1e6y_vent-20v } \\
\text { tspa00-mesh20 } \\
\text { run_control_param_SMT-v01 } \\
\text { output.times-SMT60-20 } \\
\text { SMT-1Dds-mi-sz } \\
\text { SMT-1Dds-mi-flt } \\
\text { SMT60-01-i-rst }\end{array}$ & $\begin{array}{l}1 \\
1 \\
1 \\
1 \\
1 \\
1 \\
1 \\
1\end{array}$ & \multicolumn{2}{|l|}{ Include files } \\
\hline & & user developed & & SMT60-20v.in & 1 & SMT60-20v.rep.ext & 1 \\
\hline \multirow[t]{2}{*}{$\begin{array}{l}\text { SMT } \\
\text { Postclosure }\end{array}$} & \multirow[t]{2}{*}{ SF-16 } & $\begin{array}{l}\text { readUnits output } \\
\text { heatgenAge output } \\
\text { YMESH output } \\
\mathrm{N}: \mathrm{T} 1 \\
\mathrm{~N}: \mathrm{T} 2 \\
\mathrm{~N}: \mathrm{T} 3 \\
\mathrm{E}: \mathrm{T} 1 \\
\text { NUFT output }\end{array}$ & $\begin{array}{c}\text { R-S } \\
\text { H-M1 } \\
\text { M-M1 }\end{array}$ & $\begin{array}{c}\text { SDT-1Dds-mi } \\
\text { SMT-0.3Qheat-50y_vent-20v } \\
\text { tspa00-mesh20 } \\
\text { run_control_param_SMT-v01 } \\
\text { output.times-SMT60-20 } \\
\text { SMT-1Dds-mi-sz } \\
\text { SMT-1Dds-mi-flt } \\
\text { SMT60-01-i-rst }\end{array}$ & $\begin{array}{l}1 \\
1 \\
1 \\
1 \\
1 \\
1 \\
1 \\
1\end{array}$ & \multicolumn{2}{|l|}{ Include files } \\
\hline & & user developed & & SMT60-20.in & 1 & SMT60-20.rep.ext & 1 \\
\hline
\end{tabular}


Table 11 Stratigraphic columns used in the 33 drift-scale-submodel locations. Shown is unit thickness in meters (1 of 4).

\begin{tabular}{|c|c|c|c|c|c|c|c|c|c|}
\hline \multirow{2}{*}{$\begin{array}{c}\text { Stratigraphic } \\
\text { unit }\end{array}$} & \multicolumn{9}{|c|}{ Drift-scale submodel location } \\
\hline & $11 \mathrm{cl}$ & $11 \mathrm{c2}$ & $11 \mathrm{c3}$ & $11 \mathrm{c4}$ & $12 c 1$ & $12 c 2$ & $12 c 3$ & $12 \mathrm{c4}$ & $13 c 1$ \\
\hline tcw11 & 18.662 & 0 & 0 & 0 & 0 & 0 & 23.613 & 0 & 18.662 \\
\hline tcw12 & 53.818 & 0 & 0 & 0 & 66.211 & 10.371 & 58.213 & 3.604 & 53.818 \\
\hline tcw13 & 10.957 & 0 & 0 & 0 & 6.445 & 5.83 & 4.805 & 3.896 & 10.957 \\
\hline ptn21 & 7.412 & 0 & 0 & 4.658 & 7.441 & 8.291 & 9.346 & 7.002 & 7.412 \\
\hline ptn22 & 10.195 & 10.078 & 0 & 8.467 & 7.559 & 7.061 & 6.914 & 5.537 & 10.195 \\
\hline ptn23 & 8.262 & 8.584 & 0 & 6.328 & 5.596 & 5.186 & 5.303 & 4.395 & 8.262 \\
\hline ptn24 & 32.314 & 29.355 & 28.857 & 23.35 & 19.541 & 16.055 & 12.217 & 13.389 & 32.314 \\
\hline ptn25 & 53.262 & 55.4 & 55.137 & 47.432 & 46.26 & 47.139 & 42.07 & 28.33 & 53.262 \\
\hline ptn26 & 11.016 & 11.279 & 11.396 & 11.426 & 11.865 & 12.744 & 13.008 & 10.4 & 11.016 \\
\hline tsw31 & 2.021 & 1.992 & 2.021 & 2.021 & 1.992 & 1.992 & 2.021 & 1.992 & 2.021 \\
\hline tsw32 & 45.85 & 41.044 & 40.312 & 46.729 & 45.352 & 41.572 & 37.032 & 49.482 & 45.85 \\
\hline tsw33 & 83.262 & 85.518 & 83.789 & 77.812 & 80.684 & 82.441 & 86.191 & 85.01 & 83.262 \\
\hline tsw34 & 38.076 & 38.262 & 38.144 & 35.732 & 35.586 & 35.938 & 34.864 & 31.338 & 38.076 \\
\hline tsw35 & 110.304 & 108.102 & 105.262 & 106.3 & 101.935 & 95.02 & 97.473 & 120.011 & 110.304 \\
\hline tsw36 & 9.551 & 10.453 & 13 & 8.281 & 10.586 & 15.83 & 19.5 & 19.951 & 9.551 \\
\hline tsw37 & 4.805 & 5.977 & 5.078 & 4.131 & 5.273 & 7.91 & 5.938 & 9.99 & 4.805 \\
\hline tsw38 & 14.883 & 15.508 & 15.801 & 16.523 & 16.816 & 17.539 & 19.697 & 17.373 & 14.883 \\
\hline tsw39 & 4.951 & 5.781 & 6.094 & 6.826 & 7.236 & 7.734 & 7.07 & 5.068 & 4.951 \\
\hline chivi & 0 & 0 & 0 & 0 & 0 & 0 & 0 & 0 & 0 \\
\hline ch2VI & 0 & 0 & 0 & 0 & 0 & 0 & 0 & 0 & 0 \\
\hline $\operatorname{ch} 3 \mathrm{VI}$ & 0 & 0 & 0 & 0 & 0 & 0 & 0 & 0 & 0 \\
\hline ch4VI & 0 & 0 & 0 & 0 & 0 & 0 & 0 & 0 & 0 \\
\hline ch5VI & 0 & 0 & 0 & 0 & 0 & 0 & 0 & 0 & 0 \\
\hline chize & 11.777 & 11.875 & 11.602 & 13.916 & 13.945 & 10.547 & 6.758 & 18.75 & 11.777 \\
\hline ch2Ze & 42.686 & 38.311 & 36.582 & 31.494 & 27.744 & 23.906 & 18.945 & 22.734 & 42.686 \\
\hline ch3Ze & 42.714 & 38.292 & 36.592 & 31.494 & 27.744 & 23.906 & 18.926 & 22.734 & 42.714 \\
\hline ch4Ze & 42.686 & 38.292 & 36.592 & 31.494 & 27.744 & 23.906 & 18.926 & 22.764 & 42.686 \\
\hline ch5Ze & 42.686 & 38.292 & 36.592 & 31.494 & 27.744 & 23.906 & 18.955 & 22.734 & 42.686 \\
\hline ch6 & 25.195 & 24.053 & 23.203 & 20.127 & 18.955 & 17.725 & 16.729 & 18.867 & 25.195 \\
\hline pp4 & 10.547 & 11.836 & 12.539 & 15.498 & 17.285 & 18.574 & 18.633 & 18.926 & 10.547 \\
\hline $\mathrm{pp3}=$ & 3.779 & 3.398 & 3.633 & 7.91 & 8.262 & 7.91 & 12.92 & 18.662 & 3.779 \\
\hline $\mathrm{pp} 2$ & 6.826 & 48.34 & 49.951 & 32.52 & 28.623 & 25.576 & 22.91 & 6.24 & 6.826 \\
\hline $\mathrm{pp} 1$ & 0 & 0 & 10.166 & 0 & 24.785 & 67.266 & 88.184 & 0 & 0 \\
\hline bf3 & 0 & 0 & 0 & 0 & 0 & 0 & 19.131 & 0 & 0 \\
\hline $\mathrm{bf2}$ & 0 & 0 & 0 & 0 & 0 & 0 & 0 & 0 & 0 \\
\hline
\end{tabular}


Table 11(cont'd 2 of 4).

\begin{tabular}{|c|c|c|c|c|c|c|c|c|c|}
\hline \multirow{2}{*}{$\begin{array}{c}\text { Stratigraphic } \\
\text { unit }\end{array}$} & \multicolumn{9}{|c|}{ Drift-scale submodel location } \\
\hline & $13 \mathrm{c2}$ & $13 \mathrm{c3}$ & $13 c 4$ & $14 c 1$ & $14 c 2$ & $14 c 3$ & $14 c 4$ & $14 c 5$ & $15 c 1$ \\
\hline tow11 & 0 & 31.524 & 13.096 & 0 & 0 & 0 & 0 & 34.19 & 0 \\
\hline tcw12 & 79.131 & 78.223 & 49.336 & 0 & 49.775 & 60.059 & 95.976 & 89.209 & 20.801 \\
\hline tcw13 & 4.365 & 4.482 & 4.424 & 2.285 & 5.42 & 5.391 & 5.479 & 5.039 & 4.746 \\
\hline ptn21 & 7.559 & 8.291 & 9.17 & 6.768 & 5.303 & 5.654 & 5.156 & 5.977 & 6.738 \\
\hline ptn22 & 4.834 & 4.746 & 5.537 & 6.357 & 6.064 & 4.688 & 3.867 & 2.783 & 2.812 \\
\hline ptn23 & 3.809 & 3.896 & 4.482 & 5.01 & 4.863 & 3.926 & 3.252 & 2.578 & 2.49 \\
\hline ptn24 & 10.84 & 8.936 & 9.346 & 15.264 & 9.609 & 8.584 & 7.617 & 6.738 & 6.504 \\
\hline ptn25 & 29.736 & 28.389 & 31.64 & 29.971 & 22.236 & 20.127 & 17.432 & 15.498 & 14.561 \\
\hline ptn26 & 10.225 & 12.539 & 12.92 & 9.902 & 12.451 & 13.213 & 14.15 & 15.205 & 15.41 \\
\hline tsw31 & 2.021 & 1.992 & 1.992 & 1.992 & 2.021 & 1.992 & 1.992 & 1.992 & 1.992 \\
\hline tsw32 & 46.465 & 41.894 & 38.292 & 49.922 & 52.705 & 49.16 & 47.783 & 43.184 & 38.466 \\
\hline tsw33 & 87.032 & 89.619 & 90 & 84.365 & 87.092 & 87.471 & 87.441 & 88.77 & 89.062 \\
\hline tsw34 & 31.718 & 32.129 & 32.988 & 32.912 & 32.7 & 31.654 & 30.15 & 30.342 & 31.114 \\
\hline tsw35 & 116.426 & 112.364 & 107.119 & 117.294 & 110.385 & 114.098 & 111.764 & 112.364 & 112.071 \\
\hline $\operatorname{tsw} 36$ & 21.738 & 21.914 & 20.195 & 17.637 & 33.75 & 30.586 & 31.406 & 27.646 & 23.721 \\
\hline tsw37 & 10.869 & 10.928 & 10.078 & 8.848 & 16.846 & 15.293 & 15.703 & 13.799 & 12.334 \\
\hline tsw38 & 18.867 & 21.475 & 21.875 & 17.197 & 18.779 & 20.801 & 21.006 & 23.115 & 24.658 \\
\hline tsw39 & 5.244 & 5.039 & 5.566 & 5.42 & 2.695 & 2.9 & 2.754 & 3.633 & 4.189 \\
\hline ch1VI & 0 & 0 & 0 & 0 & 0 & 0 & 0 & 10.605 & 8.027 \\
\hline ch2VI & 0 & 0 & 0 & 0 & 0 & 0 & 0 & 0 & 0 \\
\hline ch3VI & 0 & 0 & 0 & 0 & 0 & 0 & 0 & 0 & 0 \\
\hline $\operatorname{ch} 4 \mathrm{VI}$ & 0 & 0 & 0 & 0 & 0 & 0 & 0 & 0 & 0 \\
\hline ch5VI & 0 & 0 & 0 & 0 & 0 & 0 & 0 & 0 & 0 \\
\hline ch1Ze & 14.678 & 8.877 & 6.826 & 18.633 & 16.084 & 14.59 & 14.297 & 0 & 0 \\
\hline ch2Ze & 20.01 & 16.758 & 16.113 & 23.76 & 21.211 & 18.574 & 17.461 & 14.971 & 13.066 \\
\hline ch3Ze & 20.01 & 16.758 & 16.113 & 23.73 & 21.211 & 18.574 & 17.432 & 15 & 13.096 \\
\hline $\operatorname{ch} 4 \mathrm{Ze}$ & 20.01 & 16.758 & 16.143 & 23.73 & 21.211 & 18.574 & 17.461 & 14.971 & 13.096 \\
\hline ch5Ze & 20.01 & 16.758 & 16.113 & 23.73 & 21.211 & 18.545 & 17.461 & 14.971 & 13.066 \\
\hline ch6 & 18.164 & 17.666 & 17.109 & 18.896 & 17.432 & 18.252 & 18.545 & 19.453 & 19.307 \\
\hline $\mathrm{pp} 4$ & 18.838 & 16.934 & 17.139 & 18.398 & 15.352 & 13.945 & 11.631 & 9.023 & 8.027 \\
\hline $\mathrm{pp} 3$ & 19.189 & 21.943 & 20.303 & 17.051 & 2.344 & 25.4 & 28.389 & 32.461 & 33.34 \\
\hline pp2 & 15.967 & 15.82 & 18.018 & 2.08 & 0 & 18.076 & 17.871 & 15.205 & 14.561 \\
\hline pp1 & 24.316 & 71.66 & 80.449 & 0 & 0 & 0 & 15.029 & 64.893 & 55.723 \\
\hline bt3 & 0 & 0 & 20.771 & 0 & 0 & 0 & 0 & 0 & 51.533 \\
\hline bł2 & 0 & 0 & 0 & 0 & 0 & 0 & 0 & 0 & 14.766 \\
\hline
\end{tabular}


Table 11(cont'd 3 of 4)

\begin{tabular}{|c|c|c|c|c|c|c|c|c|c|}
\hline \multirow{2}{*}{$\begin{array}{l}\text { Stratigraphic } \\
\text { unit }\end{array}$} & \multicolumn{9}{|c|}{ Drift-scale submodel location } \\
\hline & $15 \mathrm{c2}$ & $15 \mathrm{c} 3$ & $15 \mathrm{c4}$ & $15 \mathrm{c} 5$ & $16 \mathrm{c1}$ & $16 \mathrm{c} 2$ & $16 c 3$ & $16 \mathrm{c4}$ & $17 \mathrm{c1}$ \\
\hline tow11 & 0 & 19.277 & 17.08 & 0 & 0 & 0 & 0 & 23.76 & 0 \\
\hline tow12 & 66.709 & 95.538 & 92.696 & 62.871 & 80.625 & 63.721 & 76.084 & 98.702 & 104.326 \\
\hline tcw13 & 5.068 & 6.006 & 6.445 & 6.855 & 4.893 & 4.951 & 4.893 & 4.482 & 5.713 \\
\hline ptn21 & 3.076 & 3.018 & 2.314 & 1.758 & 2.432 & 2.49 & 2.871 & 3.574 & 2.871 \\
\hline ptn22 & 2.08 & 2.109 & 2.197 & 2.988 & 0 & 0 & 1.846 & 2.959 & 0 \\
\hline ptn23 & 0 & 1.611 & 1.553 & 1.699 & 1.904 & 1.67 & 0 & 2.139 & 0 \\
\hline ptn24 & 7.588 & 6.182 & 6.211 & 7.09 & 4.951 & 4.512 & 6.68 & 6.592 & 4.98 \\
\hline ptn25 & 9.697 & 8.994 & 7.412 & 6.621 & 5.566 & 4.834 & 3.926 & 3.076 & 1.816 \\
\hline ptn26 & 13.652 & 14.648 & 13.945 & 14.414 & 11.396 & 10.957 & 11.191 & 11.543 & 10.225 \\
\hline tsw31 & 2.021 & 1.992 & 1.992 & 1.992 & 1.992 & 1.992 & 1.992 & 1.992 & 1.992 \\
\hline tsw32 & 43.272 & 40.312 & 38.642 & 35.098 & 37.442 & 34.952 & 33.018 & 31.172 & 28.418 \\
\hline tsw33 & 84.482 & 84.316 & 81.826 & 80.039 & 78.213 & 75.225 & 72.217 & 68.73 & 67.636 \\
\hline tsw34 & 32.602 & 32.188 & 33.535 & 34.892 & 34.643 & 37.562 & 39.189 & 42.158 & 38.012 \\
\hline tsw35 & 103.532 & 105.684 & 103.351 & 104.198 & 96.696 & 92.018 & 94.004 & 97.261 & 88.346 \\
\hline tsw36 & 35.83 & 31.777 & 32.891 & 28.664 & 40.635 & 40.693 & 35.967 & 29.332 & 38.408 \\
\hline tsw37 & 17.93 & 15.908 & 16.465 & 14.59 & 20.332 & 20.332 & 17.988 & 15.654 & 19.189 \\
\hline tsw38 & 15.527 & 18.838 & 16.992 & 19.414 & 9.844 & 9.785 & 12.07 & 14.326 & 11.191 \\
\hline tsw39 & 3.135 & 3.662 & 3.955 & 4.277 & 5.215 & 5.977 & 5.566 & 5 & 9.932 \\
\hline ch1VI & 17.783 & 14.473 & 15.293 & 11.807 & 21.885 & 22.324 & 18.691 & 13.203 & 23.379 \\
\hline ch2V1 & 15.527 & 0 & 12.451 & 10.488 & 14.15 & 13.037 & 10.4 & 0 & 13.389 \\
\hline ch3VI & 15.527 & 0 & 12.48 & 0 & 14.18 & 13.008 & 10.4 & 0 & 13.389 \\
\hline ch4VI & 0 & 0 & 0 & 0 & 0 & 0 & 0 & 0 & 13.389 \\
\hline $\operatorname{ch} 5 \mathrm{VI}$ & 0 & 0 & 0 & 0 & 14.18 & 13.037 & 10.4 & 0 & 13.389 \\
\hline $\operatorname{ch} 12 e$ & 0 & 0 & 0 & 0 & 0 & 0 & 0 & 0 & 0 \\
\hline $\operatorname{ch} 2 \mathrm{Ze}$ & 0 & 13.301 & 0 & 0 & 0 & 0 & 0 & 8.291 & 0 \\
\hline ch3Ze & 0 & 13.301 & 0 & 10.488 & 0 & 0 & 0 & 8.291 & 0 \\
\hline $\operatorname{ch} 4 \mathrm{Ze}$ & 15.527 & 13.301 & 12.451 & 10.488 & 14.18 & 13.037 & 10.4 & 8.291 & 0 \\
\hline $\operatorname{ch} 5 \mathrm{Ze}$ & 15.527 & 13.301 & 12.451 & 10.488 & 0 & 0 & 0 & 8.291 & 0 \\
\hline $\operatorname{ch} 6$ & 17.783 & 18.398 & 17.578 & 17.52 & 15.088 & 14.531 & 15.088 & 15.615 & 16.67 \\
\hline pp4 & 8.438 & 7.061 & 7.207 & 7.148 & 9.053 & 8.613 & 7.295 & 7.5 & 4.248 \\
\hline pp3 & 33.486 & 36.387 & 36.973 & 37.266 & 34.102 & 35.215 & 38.203 & 39.961 & 35.039 \\
\hline $\mathrm{pp} 2$ & 16.025 & 13.418 & 12.744 & 11.514 & 17.139 & 17.227 & 13.447 & 10.752 & 23.584 \\
\hline pp1 & 36.035 & 61.846 & 63.545 & 57.188 & 40.225 & 58.066 & 70.693 & 65.244 & 52.764 \\
\hline bf3 & 0 & 20.771 & 28.389 & 86.074 & 0 & 0 & 41.777 & 108.75 & 0 \\
\hline bf2 & 0 & 0 & 0 & 0 & 0 & 0 & 0 & 0 & 0 \\
\hline
\end{tabular}


Table 11(cont'd 4 of 4).

\begin{tabular}{|c|c|c|c|c|c|c|}
\hline \multirow{2}{*}{$\begin{array}{c}\text { Stratigraphic } \\
\text { unit }\end{array}$} & \multicolumn{6}{|c|}{ Drift-scale submodel location } \\
\hline & $17 \mathrm{c2}$ & $17 \mathrm{c3}$ & $17 c 4$ & $18 c 1$ & $18 \mathrm{c} 2$ & $18 \mathrm{cs}$ \\
\hline tcw11 & 0 & 0 & 0 & 0 & 0 & 0 \\
\hline tcw12 & 91.172 & 95.772 & 101.044 & 94.072 & 94.16 & 103.916 \\
\hline tow13 & 6.006 & 6.65 & 8.232 & 3.34 & 6.328 & 8.672 \\
\hline ptn21 & 3.135 & 3.164 & 3.311 & 2.988 & 3.164 & 3.691 \\
\hline ptn22 & 0 & 0 & 0 & 0 & 0 & 0 \\
\hline ptn23 & 0 & 0 & 1.729 & 0 & 0 & 0 \\
\hline ptn24 & 5.244 & 5.156 & 4.072 & 5.244 & 5.303 & 5.273 \\
\hline ptn25 & 2.139 & 2.109 & 2.168 & 3.926 & 3.164 & 2.168 \\
\hline ptn26 & 9.99 & 9.814 & 9.902 & 9.229 & 9.111 & 9.756 \\
\hline tsw31 & 1.992 & 1.992 & 1.992 & 1.992 & 1.992 & 1.992 \\
\hline tsw32 & 27.129 & 26.221 & 25.986 & 26.426 & 24.463 & 23.701 \\
\hline tsw33 & 66.826 & 65.625 & 64.219 & 61.895 & 59.971 & 55.43 \\
\hline tsw34 & 40.772 & 40.684 & 41.456 & 35.546 & 39.277 & 42.598 \\
\hline tsw35 & 85.996 & 84.278 & 81.848 & 67.638 & 70.34 & 73.158 \\
\hline tsw36 & 35.176 & 33.711 & 32.7 & 32.979 & 32.199 & 25.7 \\
\hline tsw37 & 17.578 & 16.875 & 17.494 & 16.465 & 15.664 & 16.406 \\
\hline tsw38 & 13.154 & 14.678 & 13.857 & 31.143 & 26.006 & 26.172 \\
\hline tsw39 & 9.229 & 9.814 & 10.586 & 7.09 & 9.756 & 11.055 \\
\hline ch1VI & 23.906 & 23.467 & 16.758 & 29.971 & 22.969 & 18.477 \\
\hline $\operatorname{ch} 2 \mathrm{VI}$ & 10.723 & 10.166 & 0 & 12.275 & 8.818 & 6.064 \\
\hline $\operatorname{ch} 3 \mathrm{VI}$ & 10.723 & 10.166 & 0 & 12.246 & 8.818 & 0 \\
\hline ch4VI & 10.723 & 10.166 & 0 & 12.275 & 8.848 & 0 \\
\hline $\operatorname{ch} 5 \mathrm{VI}$ & 10.723 & 0 & 0 & 0 & 0 & 0 \\
\hline ch1Ze & 0 & 0 & 0 & 0 & 0 & 0 \\
\hline $\operatorname{ch} 2 \mathrm{Ze}$ & 0 & 0 & 8.057 & $\overline{0}$ & 0 & 0 \\
\hline $\operatorname{ch} 3 \mathrm{Ze}$ & 0 & 0 & 8.057 & 0 & 0 & 6.123 \\
\hline ch4Ze & 0 & 0 & 8.057 & 0 & 0 & 6.094 \\
\hline ch5Ze & 0 & 10.166 & 8.057 & 12.275 & 8.818 & 6.094 \\
\hline ch6 & 15.264 & 15.264 & 14.238 & 16.553 & 15.615 & 15.41 \\
\hline pp4 & 4.834 & 4.805 & 6.24 & 7.529 & 7.031 & 7.207 \\
\hline $\mathrm{pp3}$ & 37.441 & 38.086 & 40.43 & 36.182 & 37.939 & 38.701 \\
\hline $\mathrm{pp} 2$ & 21.211 & 22.529 & 21.416 & 32.52 & 28.242 & 25.605 \\
\hline $\mathrm{pp1}$ & 77.49 & 76.377 & 69.229 & 64.57 & 79.775 & 72.92 \\
\hline bf 3 & 27.158 & 40.752 & 104.15 & 0 & 52.529 & 103.682 \\
\hline b+2 & 0 & 0 & 0 & 0 & 0 & 0 \\
\hline
\end{tabular}


Table 12 Summary of the vertical location of the repository horizon at the 33 drift-scale-submodel locations.

\begin{tabular}{|c|c|c|c|c|}
\hline $\begin{array}{l}\text { Submodel } \\
\text { location }\end{array}$ & $\begin{array}{c}\text { Host-rock } \\
\text { unit }\end{array}$ & $\begin{array}{l}\text { Repository } \\
\text { elevation (m) }\end{array}$ & $\begin{array}{l}\text { Overburden } \\
\text { thickness (m) }\end{array}$ & $\begin{array}{l}\text { Repository height } \\
\text { above water table } \\
\text { (m) }\end{array}$ \\
\hline $11 \mathrm{c1}$ & tsw35 & 1037.2 & 441.273 & 307.223 \\
\hline $11 \mathrm{c} 2$ & tsw35 & 1037.2 & 372.816 & 307.204 \\
\hline 1103 & tsw35 & 1037.2 & 355.12 & 307.223 \\
\hline $12 c 1$ & tsw35 & 1046.5 & 305.463 & 316.5 \\
\hline $12 \mathrm{c} 2$ & tsw35 & 1046.5 & 382.689 & 316.52 \\
\hline $12 c 3$ & tsw35 & 1046.5 & 345.355 & 316.52 \\
\hline $12 c 4$ & tsw35 & 1046.5 & 429.769 & 316.52 \\
\hline $13 c 1$ & tsw35 & 1055.901 & 263.275 & 325.906 \\
\hline $13 \mathrm{c} 2$ & tsw35 & 1055.901 & 356.165 & 325.906 \\
\hline $13 \mathrm{c3}$ & tsw35 & 1055.901 & 412.396 & 325.916 \\
\hline $13 c 4$ & tsw35 & 1055.901 & 387.23 & 325.925 \\
\hline $13 c 5$ & tsw35 & 1055.901 & 255.247 & 325.906 \\
\hline $14 c 1$ & tsw34 & 1065.3 & 273.441 & 335.31 \\
\hline $14 \mathrm{c2}$ & tsw35 & 1065.3 & 304.818 & 335.31 \\
\hline $14 c^{3}$ & tsw35 & 1065.3 & 343.196 & 335.309 \\
\hline $14 \mathrm{c4}$ & tsw35 & 1065.3 & 399.154 & 334.46 \\
\hline $14 c 5$ & tsw35 & 1065.3 & 333.968 & 335.31 \\
\hline $15 c 1$ & tsw34 & 1074.7 & 280.618 & 344.713 \\
\hline $15 \mathrm{c} 2$ & tsw35 & 1074.7 & 293.148 & $\mathbf{3 4 4 . 7 1 4}$ \\
\hline $15 c 3$ & tsw35 & 1074.7 & 372.904 & 344.713 \\
\hline $15 c 4$ & tsw35 & 1074.7 & 366.352 & 344.714 \\
\hline $15 c 5$ & tsw35 & 1074.7 & 353.216 & 344.713 \\
\hline $16 c 1$ & tsw35 & 1084 & 276.957 & 354.001 \\
\hline $16 \mathrm{c} 2$ & tsw35 & 1084 & 265.765 & 354 \\
\hline $16 c 3$ & tsw35 & 1084 & 312.298 & 354.001 \\
\hline $16 c 4$ & tsw36 & 1084 & 402.641 & 354 \\
\hline $17 \mathrm{c1}$ & tsw35 & 1093.401 & 278.889 & 363.406 \\
\hline $17 \mathrm{c} 2$ & tsw35 & 1093.401 & 302.327 & 363.406 \\
\hline $17 \mathrm{c3}$ & tsw35 & 1093.401 & 315.072 & 363.416 \\
\hline $17 \mathrm{c4}$ & tsw36 & 1093.401 & 361.858 & 363.426 \\
\hline $18 c 1$ & tsw35 & 1102.8 & 263.558 & 372.81 \\
\hline $18 \mathrm{c} 2$ & tsw35 & 1102.8 & 307.475 & 372.829 \\
\hline $18 c 3$ & tsw36 & 1102.8 & 343.256 & 372.81 \\
\hline
\end{tabular}


Table 13 Boundary conditions used in the drift-scale submodels (LDTH, SDT, and DDT). Note that the gasphase pressure and gas-phase air-mass fraction only apply to the LDTH submodels.

\begin{tabular}{|c|c|c|c|c|}
\hline \multirow{2}{*}{$\begin{array}{l}\text { Submodel } \\
\text { location }\end{array}$} & \multicolumn{3}{|c|}{ Ground-surface } & \multirow{2}{*}{$\begin{array}{c}\text { Water Table } \\
\text { Temperature } \\
\left({ }^{\circ} \mathrm{C}\right)\end{array}$} \\
\hline & $\begin{array}{c}\text { Temperature } \\
\left({ }^{\circ} \mathrm{C}\right)\end{array}$ & $\begin{array}{c}\text { Gas-phase } \\
\text { pressure }(\mathrm{Pa})\end{array}$ & $\begin{array}{l}\text { Gas-phase air- } \\
\text { mass fraction }\end{array}$ & \\
\hline $11 \mathrm{c1}$ & 15.84 & $9.20000 \mathrm{E}+04$ & 0.986614704 & 28.334 \\
\hline $11 \mathrm{c2}$ & 16.524 & $9.19998 \mathrm{E}+04$ & 0.986130774 & 28.408 \\
\hline 1103 & 16.385 & $9.19991 \mathrm{E}+04$ & 0.9862324 & 28.427 \\
\hline $11 c 4$ & 16.942 & $9.20000 \mathrm{E}+04$ & 0.985827684 & 28.642 \\
\hline 12c1 & 16.277 & $9.20000 \mathrm{E}+04$ & 0.986313939 & 28.725 \\
\hline $12 \mathrm{c2}$ & 16.69 & $9.20000 \mathrm{E}+04$ & 0.986012936 & 28.925 \\
\hline 1203 & 16.157 & $9.20000 \mathrm{E}+04$ & 0.986401558 & 29.344 \\
\hline 12c4 & 17.321 & $9.19999 \mathrm{E}+04$ & 0.985547543 & 29.237 \\
\hline $13 c 1$ & 16.404 & $9.20000 \mathrm{E}+04$ & 0.986227214 & 29.89 \\
\hline $13 \mathrm{c} 2$ & 15.866 & $9.20000 \mathrm{E}+04$ & 0.986609638 & 30.365 \\
\hline $13 c 3$ & 16.477 & $9.20000 E+04$ & 0.986181796 & 30.754 \\
\hline $13 c 4$ & 17.318 & $9.19998 \mathrm{E}+04$ & 0.985549271 & 29.113 \\
\hline $14 c 1$ & 17.222 & $9.20000 E+04$ & 0.985624373 & 31.393 \\
\hline $14 c 2$ & 16.714 & $9.20000 \mathrm{E}+04$ & 0.986002862 & 31.644 \\
\hline $14 \mathrm{c3}$ & 16.236 & $9.19996 \mathrm{E}+04$ & 0.986348748 & 32.026 \\
\hline $14 \mathrm{c} 4$ & 15.915 & $9.19955 \mathrm{E}+04$ & 0.986577451 & 32.369 \\
\hline $14 \mathrm{c5}$ & 16.552 & $9.20000 \mathrm{E}+04$ & 0.986120999 & 32.465 \\
\hline $15 \mathrm{c} 1$ & 16.756 & $9.20000 \mathrm{E}+04$ & 0.985973597 & 32.715 \\
\hline $15 \mathrm{c} 2$ & 16.148 & $9.20000 \mathrm{E}+04$ & 0.986413658 & 32.717 \\
\hline $15 c 3$ & 16.052 & $9.20000 \mathrm{E}+04$ & 0.986482024 & 32.631 \\
\hline $15 \mathrm{c4}$ & 16.381 & $9.20000 \mathrm{E}+04$ & 0.986247122 & 32.504 \\
\hline $15 \mathrm{c5}$ & 16.987 & $9.20000 \mathrm{E}+04$ & 0.985805094 & 32.75 \\
\hline $16 c 1$ & 16.96 & $9.19995 \mathrm{E}+04$ & 0.98582679 & 31.756 \\
\hline $16 \mathrm{c2}$ & 16.645 & $9.20000 E+04$ & 0.986057222 & 31.708 \\
\hline $16 \mathrm{c3}$ & 16.365 & $9.20000 \mathrm{E}+04$ & 0.986260235 & 31.753 \\
\hline $16 \mathrm{c} 4$ & 15.679 & $9.20000 \mathrm{E}+04$ & 0.986746073 & 31.776 \\
\hline $16 c 5$ & 16.84 & $9.20000 \mathrm{E}+04$ & 0.985918105 & 30.327 \\
\hline $17 \mathrm{c1}$ & 16.695 & $9.20000 \mathrm{E}+04$ & 0.986022949 & 30.647 \\
\hline $17 \mathrm{c2}$ & 16.432 & $9.20000 \mathrm{E}+04$ & 0.986214221 & 30.831 \\
\hline $17 \mathrm{c3}$ & 15.979 & $9.20000 \mathrm{E}+04$ & 0.986537337 & 31.193 \\
\hline $17 \mathrm{c4}$ & 16.862 & $9.20000 E+04$ & 0.985906601 & 30.044 \\
\hline $18 \mathrm{c1}$ & 16.52 & $9.20000 E+04$ & 0.986156344 & 30.552 \\
\hline $18 \mathrm{c} 2$ & 16.108 & $9.20000 \mathrm{E}+04$ & 0.986453772 & 30.968 \\
\hline $18 \mathrm{c3}$ & 16.744 & $9.20000 \mathrm{E}+04$ & 0.985992193 & 28.334 \\
\hline
\end{tabular}


Table 14 Near Field Environment and Engineered Barrier System Thermal-Hydrologic Variables Calculated with the MSTH Model

\begin{tabular}{|c|c|c|c|}
\hline $\begin{array}{l}\text { Thermal-Hydrologic } \\
\text { Variable }\end{array}$ & Drift-Scale Location & Variable Name & Section \\
\hline \multicolumn{4}{|c|}{ Near Field Environment Parameters } \\
\hline \multirow{3}{*}{ Temperature } & $\begin{array}{l}\text { Near Field Environment rock } \\
(0.2,3 \text {, and } 5 \mathrm{~m} \text { above drift and } \\
\text { along the entire repository } \\
\text { horizon })^{*}\end{array}$ & $\begin{array}{l}T_{-} X m 1 \\
T_{-} X m 2 \\
T_{-} X m 3\end{array}$ & \\
\hline & $\begin{array}{l}\text { Along Lateral Spring-line in host } \\
\text { rock }\end{array}$ & T_pillar & \\
\hline & Drift wall (perimeter average) & $T \_d w$ & \\
\hline Relative Humidity & Drift-wall (perimeter average) & $R H_{-} d w$ & \\
\hline $\begin{array}{l}\text { Liquid-phase matrix } \\
\text { saturation }\end{array}$ & Drift wall (perimeter average) & S.liq_dw & \\
\hline $\begin{array}{l}\text { Liquid-phase flux } \\
\text { (matrix) }\end{array}$ & $\begin{array}{l}\text { Near Field Environment host } \\
\text { rock }(0.2,3 \text {, and } 5 \mathrm{~m} \text { above } \\
\text { crown of drift) }\end{array}$ & $\begin{array}{l}\text { q.liq_Xm1_M } \\
\text { q.liq_Xm2_M } \\
\text { q.liq_Xm3_M }\end{array}$ & \\
\hline $\begin{array}{l}\text { Liquid-phase flux } \\
\text { (fracture) }\end{array}$ & $\begin{array}{l}\text { Near Field Environment host } \\
\text { rock }(0.2,3 \text {, and } 5 \mathrm{~m} \text { above } \\
\text { crown of drift })^{\star}\end{array}$ & $\begin{array}{l}\text { q.liq_Xm1_F } \\
\text { q.liq_Xm2_F } \\
q . l i q_{-} X m 3_{3} F\end{array}$ & \\
\hline \multicolumn{4}{|c|}{ Engineered Barrier System Parameters } \\
\hline \multirow{5}{*}{ Temperature } & Drift wall (perimeter average) & $T \_d w$ & \\
\hline & $\begin{array}{l}\text { Drip shield (perimeter average } \\
\text { and upper surface) }\end{array}$ & $\begin{array}{l}T_{-} d s \\
T_{-} d s_{-} t o p\end{array}$ & \\
\hline & $\begin{array}{l}\text { Waste Package (surface } \\
\text { average) }\end{array}$ & $T_{-} w p$ & \\
\hline & Invert (average) & $T_{-}$invert & \\
\hline & Drift-wall (perimeter average) & $R H \_d w$ & \\
\hline \multirow{5}{*}{ Relative humidity } & Drip shield (perimeter average) & $\begin{array}{l}R H \_d s \\
R H \_d s B o t\end{array}$ & \\
\hline & Waste package & $R H_{-} w p$ & \\
\hline & Invert (average) & RH_invert & \\
\hline & Drift wall (perimeter average) & S.liq_dw & \\
\hline & Invert (average) & S.liq_invert & \\
\hline \multirow{3}{*}{$\begin{array}{l}\text { Liquid-phase matrix } \\
\text { saturation }\end{array}$} & Drip shield & S.liq_ds & \\
\hline & $\begin{array}{l}\text { Drip shield (crown, upper surface } \\
\text { average, and lower side at the } \\
\text { base) }\end{array}$ & $\begin{array}{l}\text { Q.liq_dsTop } \\
\text { Q.liq_dsTop_avg } \\
\text { Q.liq_dsSide }\end{array}$ & \\
\hline & $\begin{array}{l}\text { Drift-wall (upper surface } \\
\text { perimeter average in matrix \& } \\
\text { fractures) } \\
\text { Drift-wall (bottom surface } \\
\text { perimeter average in matrix \& } \\
\text { fractures) }\end{array}$ & Q.liq_dwBot & \\
\hline
\end{tabular}




\begin{tabular}{|c|c|c|}
\hline \multirow[t]{3}{*}{ Liquid-phase flow } & $\begin{array}{l}\text { Invert (net average) } \\
\text { Net from host rock } \\
\text { Net from host rock to inner } \\
\text { Positive vertical down, inner } \\
\text { invert } \\
\text { Positive vertical down, outer } \\
\text { invert }\end{array}$ & $\begin{array}{l}\text { Q.liq_withinInvert } \\
\text { Q.liq_driftTolnvert } \\
\text { Q.liq_hstrkTolnnerinvert } \\
\text { Q.liq_posVertinnerinvert } \\
\text { Q.liq_posVertOuterinvert }\end{array}$ \\
\hline & $\begin{array}{l}\text { Drift wall (perimeter average) of } \\
\text { water vapor }\end{array}$ & Q.water.gas_dr \\
\hline & $\begin{array}{l}\text { Drift wall (perimeter average) of } \\
\text { air }\end{array}$ & Q.air.gas_dr \\
\hline \multirow[b]{2}{*}{ Gas-phase flow } & Invert (average) & Pcap_invert \\
\hline & $\begin{array}{l}\text { Drift wall (crown, in matrix and in } \\
\text { fractures) }\end{array}$ & $\begin{array}{l}\text { Pcap_M_dw } \\
\text { Pcap_F_dw }\end{array}$ \\
\hline \multirow{3}{*}{ Capillary Pressure } & Drip shield (average) & Pcap_ds \\
\hline & Drip shield (perimeter average) & x.air_ds \\
\hline & Drip shield (perimeter average) & P_ds \\
\hline $\begin{array}{l}\text { Gas-phase air-mass } \\
\text { fraction }\end{array}$ & $\begin{array}{l}\text { Drip shield (perimeter total and } \\
\text { crown) }\end{array}$ & $\begin{array}{l}\text { Qevap_dsPerim } \\
\text { Qevap_dsTop }\end{array}$ \\
\hline Gas-phase pressure & $\begin{array}{l}\text { Top half perimeter of drift wall; } \\
\text { bottom half perimeter of drift wall }\end{array}$ & $\begin{array}{l}\text { Qevap_dwTop } \\
\text { Qevap_dwBot }\end{array}$ \\
\hline $\begin{array}{l}\text { Evaporation Rate (as } \\
\text { a flow) }\end{array}$ & $\begin{array}{l}\text { Inner invert } \\
\text { Outer invert }\end{array}$ & $\begin{array}{l}\text { Qevap_invertinner } \\
\text { Qevap_invertOuter }\end{array}$ \\
\hline
\end{tabular}

- The variables designated with $X \mathrm{m1}, X \mathrm{m2}$, and $X \mathrm{~m} 3$ indicate a location of $0.2 \mathrm{~m}, 3 \mathrm{~m}$ and $5 \mathrm{~m}$, respectively above the drift crown. 
Table 15 Sequence for Combining Submodels and MSTHAC abstraction for complete MSTH simulation (Page 1 of 2), see Section 3.5.

\begin{tabular}{|c|c|c|c|c|c|c|}
\hline Step & Dimensionality & $\begin{array}{c}\text { Physical } \\
\text { Processes } \\
\end{array}$ & $\begin{array}{l}\text { Physical } \\
\text { Domain }\end{array}$ & Heat Source & $\begin{array}{l}\text { Boundary } \\
\text { Conditions }\end{array}$ & $\begin{array}{c}\text { Number of } \\
\text { Submodel } \\
\text { Runs }\end{array}$ \\
\hline \multicolumn{7}{|c|}{ NUFT submodel simultaneous execution. Smeared-heat-source mountain-scale thermal (SMT) submodel. } \\
\hline $\begin{array}{l}\text { STEP 1A } \\
\text { (steps 1A } \\
\text { through } \\
\text { 1D are } \\
\text { simultan- } \\
\text { eous) }\end{array}$ & $3 \mathrm{D}$ & $\begin{array}{l}\text { Conduction } \\
\text { only, } \\
\text { vertical } \\
\text { property } \\
\text { variation }\end{array}$ & $\begin{array}{l}\text { Repository } \\
\text { footprint plus } \\
>0.7 \mathrm{~km} \\
\text { edges, } \\
\text { surface to } 1 \\
\text { km below } \\
\text { water table }\end{array}$ & $\begin{array}{l}\text { Smeared, 1-m } \\
\text { thick, within } \\
\text { footprint, } \\
\text { reduced during } \\
\text { ventilation }\end{array}$ & $\begin{array}{l}\text { No heat flow at } \\
\text { sides, constant } \\
\text { temperature at } \\
\text { bottom and } \\
\text { surface }\end{array}$ & one \\
\hline
\end{tabular}

NUFT submodel simultaneous execution. Smeared-heat-source drift-scale thermal (SDT) submodel. At 33 selected columns. Run at 4 AML's bracketing the actual AML

\begin{tabular}{|c|c|c|c|c|c|c|}
\hline STEP $1 B$ & 10 & $\begin{array}{l}\text { Conduction } \\
\text { only in rock, } \\
\text { vertical } \\
\text { property } \\
\text { variation, } \\
\text { radiation in } \\
\text { drift, } \\
\text { approximated } \\
\text { natural } \\
\text { convection }\end{array}$ & $\begin{array}{l}\text { Surface to } \\
\text { water table }\end{array}$ & $\begin{array}{l}\text { Smeared, 1-m } \\
\text { thick, } \\
\text { reduced during } \\
\text { ventilation }\end{array}$ & $\begin{array}{l}\text { Constant } \\
\text { temperature at } \\
\text { surface, constant } \\
\text { temperature at } \\
\text { water table }\end{array}$ & 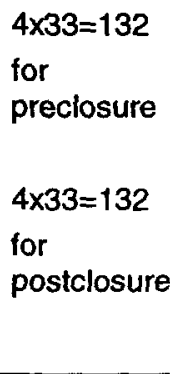 \\
\hline
\end{tabular}

NUFT submodel simultaneous execution. Line-heat-source drift-scale thermal-hydrologic (LDTH) submodel. At the same columns as in the SDT submodels. Run at 4 drift spacings creating AML's bracketing the actual AML

\begin{tabular}{|c|c|c|c|c|c|c|}
\hline STEP 1C & 20 & $\begin{array}{l}\text { Heat and mass } \\
\text { transfer, } \\
\text { vertical } \\
\text { property } \\
\text { variation, } \\
\text { radiation in } \\
\text { drift, } \\
\text { approximated } \\
\text { natural } \\
\text { convection }\end{array}$ & $\begin{array}{l}\text { Mid-drift to } \\
\text { mid-pillar, } \\
\text { surface to } \\
\text { water table }\end{array}$ & $\begin{array}{l}\text { Waste } \\
\text { package } \\
\text { volume- } \\
\text { preclosure, } \\
\text { volume under } \\
\text { drip shield- } \\
\text { postclosure, } \\
\text { line-averaged, } \\
\text { reduced during } \\
\text { ventilation }\end{array}$ & $\begin{array}{l}\text { No heat or mass } \\
\text { flow at sides, } \\
\text { constant } \\
\text { temperature, } \\
\text { pressure, and } \\
\text { relative humidity } \\
\text { at surface, } \\
\text { imposed water } \\
\text { flux map at } \\
\text { surface, constant } \\
\text { temperature, } \\
\text { pressure, and } \\
\text { saturation at water } \\
\text { table }\end{array}$ & $\begin{array}{l}4 \times 33=132 \\
\text { for } \\
\text { preclosure } \\
4 \times 33=132 \\
\text { for } \\
\text { postclosure }\end{array}$ \\
\hline \multicolumn{7}{|c|}{$\begin{array}{l}\text { NUFT submodel simultaneous execution. } \\
\text { location. }\end{array}$} \\
\hline STEP 1D & $3 D$ & $\begin{array}{l}\text { Conduction } \\
\text { only in rock, } \\
\text { vertical } \\
\text { property } \\
\text { variation, } \\
\text { radiation in } \\
\text { drift, radiation } \\
\text { under drip } \\
\text { shield, no } \\
\text { natural conv }\end{array}$ & $\begin{array}{l}\text { Mid-drift to } \\
\text { mid-pillar, } \\
\text { surface to } \\
\text { water table, } \\
\text { No drip shield } \\
\text { during } \\
\text { preclosure }\end{array}$ & $\begin{array}{l}\text { Eight full and } \\
\text { two half Waste } \\
\text { packages (of } \\
\text { same } \\
\text { diameter, but } \\
\text { individual } \\
\text { lengths and } \\
\text { thermal } \\
\text { powers), } \\
\text { reduced during } \\
\text { ventilation }\end{array}$ & $\begin{array}{l}\text { No heat flow at } \\
\text { sides, constant } \\
\text { temperature at } \\
\text { bottom, constant } \\
\text { temperature at } \\
\text { surface }\end{array}$ & $\begin{array}{l}\text { one for } \\
\text { preclosure } \\
\text { one for } \\
\text { postclosure }\end{array}$ \\
\hline
\end{tabular}


Table 15 (cont Page 2 of 2)

\begin{tabular}{|c|c|c|c|c|c|c|}
\hline Step & Dimensionality & $\begin{array}{c}\text { Physical } \\
\text { Processes }\end{array}$ & $\begin{array}{c}\text { Physical } \\
\text { Domain }\end{array}$ & Heat Source & $\begin{array}{c}\text { Boundary } \\
\text { Conditions }\end{array}$ & $\begin{array}{c}\text { Number of } \\
\text { Submodel } \\
\text { Runs }\end{array}$ \\
\hline
\end{tabular}

STEP 2 Tabulate SMT predicted host rock temperature histories at each column and time step to additional locations within the footprint. HTOM: 671 locations, LTOM: 762 locations

STEP 3 Tabulate SDT predicted host rock temperature histories at each of 33 submodel locations within the footprint.

STEP 4 Build Time-varying Effective AML Curves at the 33 drift-scale submodel locations. These curves map the variation of the AML loading with time at each column location. At each submodel location and at each time step, determine the interpolated AML in the SDT array to match the local SMT temperature.

STEP 5 Build Time-varying Effective AML Curves at the 671 or 762 repository subdomains. At each location and selected time steps, determine the interpolated AML in the SDT array to match the local SMT temperature. HTOM: 671 curves, LTOM: 762 curves

STEP 6 Calculate LMTH drift-wall temperature at $\mathbf{6 7 1}$ or $\mathbf{7 6 2}$ repository subdomains. Interpolate temperatures at each zone and time step to additional locations within the footprint. At each of the locations and selected time steps, use the Time-varying Effective AML (from Step 5) to interpolate temperatures among the AML's.

Result: Temperatures at 671 or 762 subdomains, consistent with edge effects, infiltration map and drift layout, but not including three-dimensional WP-to-WP variations. These are termed line-source, mountain-scale thermalhydrologic (LMTH) temperatures.

HTOM: 671 subdomains, LTOM: 762 subdomains

STEP 7 Calculate 6710 or 7620 DMTH drift-wall temperatures at the 671 or 762 repository subdomains. At each of the locations and selected time steps, use the Effective AML (from Step 4) to interpolate among the AML's. DDT submodel results are used to interpolate the axial deviation histories for the 10 waste packages. These deviation histories are used to adjust the LMTH temperatures from Step 6, producing discrete-source, mountain-scale, thermal-hydrologic (DMTH) temperature histories at all locations and positions within locations

Result: Temperatures across the entire domain and time history. HTOM: 671 subdomains, 6710 drift-wall temps; LTOM: 762 subdomains, 7620 drift-wall temps

STEP 8 Build Time-varying Specific AML Curves at the $\mathbf{3 3}$ drift-scale submodel locations. These curves map the variation of the AML loading with time at each column location. At each submodel location and at each time step, determine the interpolated AML in the LDTH array to match the local DMTH temperature.

STEP 9 Build Time-varying Specific AML Curves at the 671 or 762 repository subdomains. At each location and selected time steps, determine the interpolated AML in the LDTH array to match the local DMTH temperature.

HTOM: 671 curves, LTOM: 762 curves

STEP 10 Interpolate final DMTH output variables. At each location DMTH variables are found by interpolating LDTH variables onto the Time-varying Specific AML of MSTHAC Step 4B.

See Table 14 for a list of the variables computed for the DMTH model.

See Figure 3 and Figure 28.

NOTES: Selected times and positions refer to positions and times used in downstream models. $A M L=$ areal mass loading; $W P=$ waste package 
Table 16 Invert material properties.

\begin{tabular}{|l|c|c|c|c|}
\hline \multicolumn{1}{|c|}{ Property } & Units & $\begin{array}{c}\text { Crushed Tuff } \\
\text { Invert }\end{array}$ & $\begin{array}{c}\text { Waste } \\
\text { Package }\end{array}$ & $\begin{array}{c}\text { Drip } \\
\text { Shield }\end{array}$ \\
\hline Permeability & $\mathrm{m}^{2}$ & $6.15 \times 10^{-10}$ & NA & NA \\
\hline Porosity & Fraction & 0.545 & NA & NA \\
\hline $\begin{array}{l}\text { Van } \\
\text { Genuchten } \\
\alpha\end{array}$ & $1 / \mathrm{Pa}$ & $1.2232 \times 10^{-3}$ & NA & NA \\
\hline $\begin{array}{l}\text { Van } \\
\text { Genuchten } \beta\end{array}$ & dimensionless & 2.7 & NA & NA \\
\hline $\begin{array}{l}\text { Residual } \\
\text { Saturation }\end{array}$ & Fraction & 0.092 & NA & NA \\
\hline $\begin{array}{l}\text { Grain } \\
\text { Density }\end{array}$ & Kg/m 3 & $2530^{3}$ & 8189.2 & 4501.72 \\
\hline $\begin{array}{l}\text { Grain } \\
\text { Specific } \\
\text { Heat }\end{array}$ & $\mathrm{J} / \mathrm{Kg}-\mathrm{K}$ & $948^{3}$ & 488.86 & 551.32 \\
\hline $\begin{array}{l}\text { Conductivity } \\
\text { Won }\end{array}$ & W/m-K & $\begin{array}{l}1.52 \text { (upper layer) } \\
0.15 \text { (lower layer) }^{2}\end{array}$ & 14.42 & 20.55 \\
\hline Emissivity & dimensionless & 0.93 & $0.80^{1}$ & 0.63 \\
\hline
\end{tabular}

Sources:

'(Avallone, E.A. and Baumeister, T., III, ed. 1987, p. 4-68). The value from carbon steel is used as an approximation.

2 See Section 3.2.8.

${ }^{3}$ See Section 3.2.7. 
Table 17 Matrix properties of stratigraphic units.

\begin{tabular}{|c|c|c|c|c|c|c|}
\hline Unit & Permeability & Porosity & $\begin{array}{c}\text { Van } \\
\text { Genuchten } \alpha\end{array}$ & Van Genuchten & $\begin{array}{l}\text { Residual } \\
\text { Saturation }\end{array}$ & $\begin{array}{c}\text { Satiated } \\
\text { Saturation }\end{array}$ \\
\hline & $\left(\mathrm{m}^{2}\right)$ & (Fraction) & $\left(\mathrm{Pa}^{-1}\right)$ & & (Fraction) & (Fraction) \\
\hline tcw11 & $3.86 \mathrm{E}-15$ & 0.253 & 4.00E-05 & 0.47 & 0.07 & 1 \\
\hline tcw12 & $2.74 \mathrm{E}-19$ & 0.082 & 1.81E-05 & 0.241 & 0.19 & 1 \\
\hline tcw13 & $9.23 \mathrm{E}-17$ & 0.203 & $3.44 E-06$ & 0.398 & 0.31 & 1 \\
\hline ptn21 & $9.90 \mathrm{E}-13$ & 0.387 & $1.01 \mathrm{E}-05$ & 0.176 & 0.23 & 1 \\
\hline ptn22 & $2.65 \mathrm{E}-12$ & 0.439 & $1.60 \mathrm{E}-04$ & 0.326 & 0.16 & 1 \\
\hline ptn23 & $1.23 E-13$ & 0.254 & $5.58 E-06$ & 0.397 & 0.08 & 1 \\
\hline $\mathrm{ptn} 24$ & $7.86 \mathrm{E}-14$ & 0.411 & $1.53 E-04$ & 0.225 & 0.14 & 1 \\
\hline ptn25 & $7.00 \mathrm{E}-14$ & 0.499 & 5.27E-05 & 0.323 & 0.06 & 1 \\
\hline ptn26 & $2.21 \mathrm{E}-13$ & 0.492 & 2.49E-04 & 0.285 & 0.05 & 1 \\
\hline tsw31 & $6.32 \mathrm{E}-17$ & 0.053 & $3.61 E-05$ & 0.303 & 0.22 & 1 \\
\hline tsw32 & 5.83E-16 & 0.157 & $3.61 E-05$ & 0.333 & 0.07 & 1 \\
\hline tsw33 & $3.08 \mathrm{E}-17$ & 0.154 & 2.13E-05 & 0.298 & 0.12 & 1 \\
\hline tsw34 & 4.07E-18 & 0.11 & $3.86 E-06$ & 0.291 & 0.19 & 1 \\
\hline tsw35 & $3.04 \mathrm{E}-17$ & 0.131 & 6.44E-06 & 0.236 & 0.12 & 1 \\
\hline tsw36 & $5.71 \mathrm{E}-18$ & 0.112 & $3.55 E-06$ & 0.38 & 0.18 & 1 \\
\hline tsw37 & $4.49 \mathrm{E}-18$ & 0.094 & 5.33E-06 & 0.425 & 0.25 & 1 \\
\hline tsw38 & $4.53 \mathrm{E}-18$ & 0.037 & $6.94 \mathrm{E}-06$ & 0.324 & 0.44 & 1 \\
\hline tsw39 & $5.46 \mathrm{E}-17$ & 0.173 & $2.29 E-05$ & 0.38 & 0.29 & 1 \\
\hline $\operatorname{ch} 12$ & $1.96 \mathrm{E}-19$ & 0.288 & 2.68E-07 & 0.316 & 0.33 & 1 \\
\hline chiv & $9.90 \mathrm{E}-13$ & 0.273 & 1.43E-05 & 0.35 & 0.03 & 1 \\
\hline $\operatorname{ch} 2 v$ & $9.27 \mathrm{E}-14$ & 0.345 & $5.13 E-05$ & 0.299 & 0.07 & 1 \\
\hline $\operatorname{ch} 3 v$ & $9.27 E-14$ & 0.345 & 5.13E-05 & 0.299 & 0.07 & 1 \\
\hline ch4v & $9.27 E-14$ & 0.345 & $5.13 E-05$ & 0.299 & 0.07 & 1 \\
\hline ch5v & $9.27 \mathrm{E}-14$ & 0.345 & 5.13E-05 & 0.299 & 0.07 & 1 \\
\hline ch2z & $6.07 E-18$ & 0.331 & $3.47 E-06$ & 0.244 & 0.28 & 1 \\
\hline $\operatorname{ch} 3 z$ & $6.07 E-18$ & 0.331 & $3.47 E-06$ & 0.244 & 0.28 & 1 \\
\hline $\operatorname{ch} 4 z$ & 6.07E-18 & 0.331 & $3.47 \mathrm{E}-06$ & 0.244 & 0.28 & 1 \\
\hline $\operatorname{ch} 5 z$ & $6.07 E-18$ & 0.331 & 3.47E-06 & 0.244 & 0.28 & 1 \\
\hline $\operatorname{ch} 6$ & 4.23E-19 & 0.266 & 3.38E-07 & 0.51 & 0.37 & 1 \\
\hline pp4 & 4.28E-18 & 0.325 & $1.51 \mathrm{E}-07$ & 0.676 & 0.28 & 1 \\
\hline $\mathrm{pp} 3$ & $2.56 E-14$ & 0.303 & 2.60E-05 & 0.363 & 0.1 & 1 \\
\hline $\mathrm{pp} 2$ & $1.57 E-16$ & 0.263 & 2.67E-06 & 0.369 & 0.18 & 1 \\
\hline pp1 & $6.40 \mathrm{E}-17$ & 0.28 & 1.14E-06 & 0.409 & 0.3 & 1 \\
\hline bf3 & $2.34 E-14$ & 0.115 & 4.48E-06 & 0.481 & 0.11 & 1 \\
\hline bf2 & $2.51 \mathrm{E}-17$ & 0.259 & $1.54 E-07$ & 0.569 & 0.18 & 1 \\
\hline
\end{tabular}

DTN: LB990861233129.001 
Table 18 Fracture properties of stratigraphic units.

\begin{tabular}{|c|c|c|c|c|c|c|c|c|c|c|c|c|c|}
\hline Unit & $\begin{array}{c}\text { Lower Intiluration } \\
\text { Permeability }\end{array}$ & $\begin{array}{l}\text { Base Case } \\
\text { Infiltration } \\
\text { Pemeability }\end{array}$ & $\begin{array}{c}\text { Uppor } \\
\text { Infilltration } \\
\text { Permeability }\end{array}$ & Porosity & $\begin{array}{c}\text { Lower } \\
\text { Infiltration } \\
\text { Van } \\
\text { Genuchten } \alpha \\
\end{array}$ & $\begin{array}{c}\text { Base Case } \\
\text { Infiltration Var } \\
\text { Genuchten } \alpha \\
\end{array}$ & $\begin{array}{c}\text { Upper } \\
\text { Intititration Van } \\
\text { Genuchten } \alpha \\
\end{array}$ & $\begin{array}{c}\text { Van } \\
\text { Genuchten } \beta\end{array}$ & $\begin{array}{l}\text { Residual } \\
\text { Saturation }\end{array}$ & $\begin{array}{c}\text { Satiated } \\
\text { Saturation }\end{array}$ & $\begin{array}{c}\text { Active } \\
\text { Fracture } \\
\text { Parameter }\end{array}$ & Frequency & $\begin{array}{c}\text { Fracture to } \\
\text { matrix } \\
\text { area }\end{array}$ \\
\hline & $\left(m^{2}\right)$ & $\left(\mathrm{m}^{2}\right)$ & $\left(m^{2}\right)$ & & $\left(\mathrm{Pa}^{-1}\right)$ & $\left(\mathrm{Pa}^{-1}\right)$ & $\left(\mathrm{Pa}^{-1}\right)$ & & (Fraction) & (Fraction) & & $(1 / \mathrm{m})$ & $\left(\mathrm{m}^{2} / \mathrm{m}^{3}\right)$ \\
\hline Icw11 & SAB & $2.41 E-12$ & $S A B$ & 0.028 & SAB & $3.15 \mathrm{E}-03$ & SAB & 0.627 & 0.01 & 1 & 0.30 & 0.92 & 1.56 \\
\hline Icw12 & $S A B$ & $1.00 \mathrm{E}-10$ & $S A B$ & 0.02 & $\mathrm{SAB}$ & 2.13E-03 & $S A B$ & 0.613 & 0.01 & 1 & 0.30 & 1.91 & 13.39 \\
\hline ICw13 & SAB & $5.42 \mathrm{E}-12$ & SAB & 0.015 & $S A B$ & $1.26 \mathrm{E}-03$ & $S A B$ & 0.607 & 0.01 & 1 & 0.30 & 2.79 & 3.77 \\
\hline ptn21 & SAB & 1.86E-12 & $S A B$ & 0.011 & SAB & $1.68 \mathrm{E}-03$ & SAB & 0.58 & 0.01 & 1 & 0.09 & 0.67 & 1.00 \\
\hline $\operatorname{pin} 22$ & SAB & $2.00 \mathrm{E}-11$ & SAB & 0.012 & SAB & 7.68E-04 & SAB & 0.58 & 0.01 & 1 & 0.09 & 0.46 & 1.41 \\
\hline pin23 & SAB & $2.60 \mathrm{E}-13$ & $\widehat{S A B}$ & 0.0025 & SAB & $9.23 \mathrm{E}-04$ & SAB & 0.61 & 0.01 & 1 & 0.09 & $0 . \overline{57}$ & 1.75 \\
\hline$\rho \ln 24$ & $\mathrm{SAB}$ & $4.67 \mathrm{E}-13$ & SAB & 0.012 & $\mathrm{SAB}$ & 3.37E-03 & $\mathrm{SAB}$ & 0.623 & 0.01 & 1 & 0.09 & 0.46 & 0.34 \\
\hline ptn25 & SAB & $7.03 \mathrm{E}-13$ & SAB & 0.0062 & SAB & $6.33 E-04$ & $S A B$ & 0.644 & 0.01 & 1 & 0.09 & 0.52 & 1.09 \\
\hline ptin26 & SAB & 4.44E-13 & $S A B$ & 0.0036 & SAB & $2.79 \mathrm{E}-04$ & SAB & 0.552 & 0.01 & 1 & 0.09 & 0.97 & 3.56 \\
\hline isw31 & SAB & $3.21 \mathrm{E}-11$ & SAB & 0.0055 & SAB & $2.49 \mathrm{E}-04$ & SAB & 0.566 & 0.01 & 1 & 0.06 & 2.17 & 3.86 \\
\hline $15 \times 32$ & $1.41 E_{-12}$ & $2.51 \mathrm{E}-12$ & 1.41E-12 & 0.0095 & $S A B$ & 1.27E-03 & $S A B$ & 0.608 & 0.01 & 1 & 0.41 & 1.12 & 3.21 \\
\hline t5w33 & $4.91 E-13$ & $8.79 E-13$ & 8.33E-13 & 0.0066 & SAB & $1.46 \mathrm{E}-03$ & SAB & 0.608 & 0.01 & 1 & 0.41 & 0.81 & 4.44 \\
\hline $15 w 34$ & $5.35 E-13$ & 3.68E-13 & $3.76 E-13$ & 0.01 & SAB & $5.16 \mathrm{E}-04$ & SAB & 0.608 & 0.01 & 1 & 0.41 & 4.32 & 13.54 \\
\hline $15 \times 35$ & $2.41 E-12$ & $2.38 E-12$ & $3.80 E-12$ & 0.018 & $0.83 E-05$ & 8.23E-05 & 4.94E-05 & 0.611 & 0.01 & 1 & 0.41 & 3.16 & 9.68 \\
\hline tamB6 & $1.60 \mathrm{E}-12$ & 1.38E-12 & $1.36 \mathrm{E}-12$ & 0.015 & SAB & 7.84E-04 & $S A B$ & 0.61 & 0.01 & 1 & 0.41 & 4.02 & 12.31 \\
\hline 58 157 & $1.60 \mathrm{E}-12$ & 1.38E-12 & $1.36 \mathrm{E}-12$ & 0.015 & SAB & $7.84 E-04$ & $S A B$ & 0.61 & 0.01 & 1 & 0.41 & 4.02 & 12.31 \\
\hline $15 m 38$ & SAB & 5.92E-13 & SAB & 0.012 & SAB & $4.87 \mathrm{E}-04$ & SAB & 0.612 & 0.01 & 1 & 0.41 & 4.36 & 13.34 \\
\hline $15 \times 39$ & $S A B$ & $4.57 \mathrm{E}-13$ & $S A B$ & 0.0046 & SAB & 9.63E-04 & $\mathrm{SAB}$ & 0.634 & 0.01 & 1 & 0.41 & 0.96 & 2.95 \\
\hline $\operatorname{ch} 12$ & $S A B$ & $3.40 \mathrm{E}-13$ & SAB & 0.00017 & SAB & 1.43E-03 & $\mathbf{S A B}$ & 0.631 & 0.01 & 1 & 0.10 & 0.04 & 0.11 \\
\hline chiv & SAB & $1.84 \mathrm{E}-12$ & $\mathrm{SAB}$ & 0.00069 & $S A B$ & $1.09 \mathrm{E}-03$ & SAB & 0.624 & 0.01 & 1 & 0.13 & 0.10 & 0.30 \\
\hline chen & SAB & $2.69 \mathrm{E}-13$ & $S A B$ & 0.00089 & $S A B$ & $5.18 E-04$ & SAB & 0.628 & 0.01 & 1 & 0.13 & 0.14 & 0.43 \\
\hline $\cot 3 v$ & SAB & $2.69 \mathrm{E}-13$ & SAB & 0.00089 & SAB & $5.18 \mathrm{E}-04$ & SAB & 0.628 & 0.01 & 1 & 0.13 & 0.14 & 0.43 \\
\hline $\operatorname{con} 4 v$ & SAB & $2.89 E-13$ & SAB & 0.00089 & SAB & $5.18 E-04$ & SAB & 0.628 & 0.01 & 1 & 0.13 & 0.14 & 0.43 \\
\hline $\cos 5 v$ & SAB & $2.89 \mathrm{E}-13$ & $S A B$ & 0.00089 & $\mathrm{SAB}$ & $5.18 \mathrm{E}-04$ & SAB & 0.628 & 0.01 & 1 & 0.13 & 0.14 & 0.43 \\
\hline $\operatorname{cth} 22$ & SAB & $3.12 E-14$ & SAB & 0.00043 & SAB & 4.B8E-04 & SAB & 0.598 & 0.01 & 1 & 0.10 & 0.14 & 0.43 \\
\hline $\operatorname{ch} 3 z$ & SAB & 3.12E-14 & SAB & 0.00043 & SAB & 4.88E-04 & SAB & 0.598 & 0.01 & 1 & 0.10 & 0.14 & 0.43 \\
\hline $\cos 4 z$ & $S A B$ & $3.12 E-14$ & $S A B$ & 0.00043 & $S A B$ & 4.80E-04 & SAB & 0.598 & 0.01 & 1 & 0.10 & 0.14 & 0.43 \\
\hline $\operatorname{ch} 5 z$ & SAB & 3.12E-14 & $S A B$ & 0.00043 & SAB & $4.88 \mathrm{E}-04$ & SAB & 0.598 & 0.01 & 1 & 0.10 & 0.14 & 0.43 \\
\hline $\operatorname{ch} 6$ & SAB & $1.67 E-14$ & $S A B$ & 0.00017 & $S A B$ & 7.49E-04 & SAB & 0.604 & 0.01 & 1 & 0.10 & 0.04 & 0.11 \\
\hline DPA & SAB & $3.84 E-14$ & SAB & 0.00043 & SAB & $5.72 E-04$ & SAB & 0.627 & 0.01 & 1 & 0.10 & 0.14 & 0.43 \\
\hline pp3 & $\mathrm{SAB}$ & $7.60 \mathrm{E}-12$ & $\mathrm{SAB}$ & 0.0011 & SAB & $8.73 \mathrm{E}-04$ & SAB & 0.655 & 0.01 & 1 & 0.46 & 0.20 & 0.61 \\
\hline$p p 2$ & SAB & $1.38 \mathrm{E}-13$ & SAB & 0.0011 & SAB & 1.21E-03 & SAB & 0.606 & 0.01 & 1 & 0.46 & 0.20 & 0.61 \\
\hline pp1 & $\mathrm{SAB}$ & 1.12E-13 & SAB & 0.00043 & $S A B$ & 5.33E-04 & SAB & 0.622 & 0.01 & 1 & 0.10 & 0.14 & 0.43 \\
\hline b\$3 & $\mathrm{SAB}$ & $4.08 E-13$ & SAB & 0.0011 & SAB & $9.95 \mathrm{E}-04$ & $S A B$ & 0.624 & 0.01 & 1 & 0.46 & 0.20 & 0.61 \\
\hline 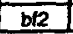 & $\mathrm{SAB}$ & $1.30 \mathrm{E}-14$ & $\widehat{\mathrm{SAB}}$ & 0.00043 & SAB & $5.42 \mathrm{E}-0.04$ & SAB & 0.608 & 0.01 & 1 & 0.10 & 0.14 & 0.43 \\
\hline
\end{tabular}

SAB indicates that the values are the same as for Base Case infiltration.

DTN: LB990861233129.001 
Table 19 Thermal properties of stratigraphic units.

\begin{tabular}{|c|c|c|c|c|}
\hline Model Layer & Rock Grain Density & Rock Grain Specific & Dry Conductivity & Wet Conductivity \\
\hline & $\mathrm{Kg} / \mathrm{m}^{3}$ & Heat $(\mathrm{J} / \mathrm{Kg} \mathrm{K})$ & $\mathrm{W} / \mathrm{m} \mathrm{K}$ & $\mathrm{W} / \mathrm{m} \mathrm{K}$ \\
\hline tcw11 & 2550 & 823 & 1.6 & 2 \\
\hline tcw12 & 2510 & 851 & 1.24 & 1.81 \\
\hline $\operatorname{tcw13}$ & 2470 & 857 & 0.54 & 0.98 \\
\hline ptn21 & 2380 & 1040 & 0.5 & 1.07 \\
\hline ptn22 & 2340 & 1080 & 0.35 & 0.5 \\
\hline ptn23 & 2400 & 849 & 0.44 & 0.97 \\
\hline ptn24 & 2370 & 1020 & 0.46 & 1.02 \\
\hline ptn25 & 2260 & 1330 & 0.35 & 0.82 \\
\hline ptn26 & 2370 & 1220 & 0.23 & 0.67 \\
\hline tsw31 & 2510 & 834 & 0.37 & 1 \\
\hline tsw32 & 2550 & 866 & 1.06 & 1.62 \\
\hline tsw33 & 2510 & 882 & 0.79 & 1.68 \\
\hline tsw34 & 2530 & 948 & 1.56 & 2.33 \\
\hline tsw35 & 2540 & 900 & 1.27 & 1.87 \\
\hline tsw36 & 2560 & 865 & 1.42 & 1.84 \\
\hline tsw37 & 2560 & 865 & 1.42 & 1.84 \\
\hline tsw38 & 2360 & 984 & 1.69 & 2.08 \\
\hline tsw39 & 2360 & 984 & 1.69 & 2.08 \\
\hline $\operatorname{ch} 12$ & 2310 & 1060 & 0.7 & 1.31 \\
\hline ch1v & 2310 & 1060 & 0.7 & 1.31 \\
\hline $\operatorname{ch} 2 v$ & 2240 & 1200 & 0.58 & 1.17 \\
\hline ch3v & 2240 & 1200 & 0.58 & 1.17 \\
\hline $\operatorname{ch} 4 \mathrm{v}$ & 2240 & 1200 & 0.58 & 1.17 \\
\hline ch5v & 2240 & 1200 & 0.58 & 1.17 \\
\hline $\operatorname{ch} 2 z$ & 2350 & 1150 & 0.61 & 1.2 \\
\hline $\operatorname{ch} 3 z$ & 2350 & 1150 & 0.61 & 1.2 \\
\hline $\operatorname{ch} 4 z$ & 2350 & 1150 & 0.61 & 1.2 \\
\hline ch5z & 2350 & 1150 & 0.61 & 1.2 \\
\hline ch6 & 2440 & 1170 & 0.73 & 1.35 \\
\hline $\mathrm{pp} 4$ & 2410 & 577 & 0.62 & 1.21 \\
\hline pp3 & 2580 & 841 & 0.66 & 1.26 \\
\hline $\mathrm{pp} 2$ & 2580 & 841 & 0.66 & 1.26 \\
\hline pp1 & 2470 & 635 & 0.72 & 1.33 \\
\hline bf3 & 2570 & 763 & 1.41 & 1.83 \\
\hline bf2 & 2410 & 633 & 0.74 & 1.36 \\
\hline
\end{tabular}

DTN: LB991091233129.006 
Table 20 Sensitivity of In-Drift Thermal-Hydrologic Performance to Uncertainties and Parameters

\begin{tabular}{|c|c|c|c|c|c|}
\hline $\begin{array}{l}\text { Results } \\
\text { Section }\end{array}$ & $\begin{array}{c}\text { Parameter or } \\
\text { Uncertainty }\end{array}$ & $\begin{array}{l}\text { Range of Parameter } \\
\text { or Uncertainty }\end{array}$ & Base Case & $\begin{array}{c}\text { Performance } \\
\text { Measure }\end{array}$ & $\begin{array}{l}\text { Effect of Parameter or } \\
\text { Uncertainty Range on } \\
\text { Performance Measure }\end{array}$ \\
\hline 6.1 .1 & $\begin{array}{l}\text { Gas storage in } \\
\text { lithophysal } \\
\text { cavities }\end{array}$ & Include or Exclude & Exclude & $\begin{array}{l}\text { Fraction of } \\
\text { vaporizing liquid } \\
\text { included in model }\end{array}$ & $\begin{array}{l}0.1 \% \text { over-estimate of } \\
\text { volume of vaporized } \\
\text { liquid displaced to } \\
\text { adjacent zones }\end{array}$ \\
\hline $\begin{array}{l}6.1 .2 \\
\text { HTOM, } \\
\text { LTOM }\end{array}$ & $\begin{array}{l}\text { Buoyant gas- } \\
\text { phase } \\
\text { convection (drift- } \\
\text { ) }\end{array}$ & $\begin{array}{l} \pm 2 \text { standard } \\
\text { deviations } \\
k_{b}=0.15-38 \text { Darcy, } \\
\text { Half-pillar scale }\end{array}$ & $k_{b}=2.4$ & $\begin{array}{l}\text { Percolation flux, } \\
\text { DW \& DS T }\end{array}$ & $\begin{array}{l}\text { HTOM boiling period: } \\
\text { up to double the heat- } \\
\text { mobilized liquid-phase } \\
\text { flux; peak temperature } \\
\text { range of } 11^{\circ} \mathrm{C} \text {; boiling } \\
\text { period range of } 50 \\
\text { years }\end{array}$ \\
\hline $\begin{array}{l}6.1 .3 \\
\text { HTOM, } \\
\text { LTOM }\end{array}$ & $\begin{array}{l}\text { Host rock } \\
\text { permeability }\end{array}$ & $k_{b}=0.15-38$ Darcy & $\begin{array}{l}\text { Mean } k_{b} \text { (unit } \\
\text { dependent) } \\
K_{b}=2.38\end{array}$ & $\begin{array}{l}\text { DW T \& RH, } \\
\text { DS T \& RH, } \\
\text { INVUS }\end{array}$ & $\mathrm{DW} T 11^{\circ} \mathrm{C}$ \\
\hline $\begin{array}{l}6.1 .4 \\
\text { HTOM, } \\
\text { LTOM }\end{array}$ & $\begin{array}{l}\text { Host rock } \\
\text { thermal } \\
\text { conductivity }\end{array}$ & $\begin{array}{l}K_{t h}=1.13-2.02 \text { wet } \\
K_{t h}=0.64-1.54 \text { dry }\end{array}$ & $\begin{array}{l}\text { Mean: } \\
1.87 \text { wet; } \\
1.27 \mathrm{dry}\end{array}$ & $\begin{array}{l}\text { DW T \& RH, } \\
\text { DS T \& RH, } \\
\text { INV } \mathrm{S}\end{array}$ & $\begin{array}{l}\text { DW \& DS T } \sim 85^{\circ} \mathrm{C} \\
\text { range, HTOM; } \\
\text { DW \& DS T } 220^{\circ} \mathrm{C} \\
\text { range, LTOM }\end{array}$ \\
\hline $\begin{array}{l}6.1 .5 \\
\text { HTOM, } \\
\text { LTOM }\end{array}$ & $\begin{array}{l}\text { Lithophysal } \\
\text { porosity } \\
\text { (combined } \\
\text { influence on } \\
\text { thermal } \\
\text { conductivity and } \\
\text { heat capacity) } \\
\end{array}$ & O to $25 \%$ & $\begin{array}{l}\text { Mean } \\
\text { porosity: } \\
12.5 \%\end{array}$ & $\begin{array}{l}\text { DW T \& RH, } \\
\text { DS T \& RH, } \\
\text { INVuS }\end{array}$ & $\begin{array}{l}\text { DW \& DS T }-100^{\circ} \mathrm{C} \\
\text { range, HTOM; } \\
\text { DW \& DS T }-25^{\circ} \mathrm{C} \\
\text { range, LTOM }\end{array}$ \\
\hline $\begin{array}{l}6.1 .6 \\
\text { LTOM }\end{array}$ & $\begin{array}{l}\text { WP capacity } \\
\text { drift spacing } \\
\text { WP spacing }\end{array}$ & $\begin{array}{l}\text { 16-21 PWR SNFA's } \\
81-97 \mathrm{~m} \text { drift spacing } \\
0.1-2 \mathrm{~m} \text { average WP } \\
\text { spacing }\end{array}$ & $\begin{array}{l}21 \text { SNFA's, } \\
81 \mathrm{~m} \text { drift } \\
\text { spacing } \\
0.1 \mathrm{~m} \text { WP } \\
\text { spacing }\end{array}$ & Peak WP T & $7^{\circ} \mathrm{C}$ range \\
\hline $\begin{array}{l}6.1 .7 \\
\text { HTOM, } \\
\text { LTOM }\end{array}$ & $\begin{array}{l}\text { Pre-closure } \\
\text { dryout }\end{array}$ & $\begin{array}{l}6 \text { months to dryout } 3 \\
\mathrm{~m} \text { rock, } \\
10 \mathrm{~mm} / \mathrm{yr} \text { flux } \\
\text { entering from } 11.5 \mathrm{~m} \\
\text { wide region; } \\
\text { Calculated dryout }\end{array}$ & $\begin{array}{l}\text { No water } \\
\text { entering drift }\end{array}$ & $\begin{array}{l}\mathrm{RH} \text { at } 500 \mathrm{~m} \text { in } \\
\text { ventilation air, } \\
\mathrm{kW} / \mathrm{m} \text { latent heat } \\
\text { removal, } \\
\text { DS T postclosure }\end{array}$ & $\begin{array}{l}9.9 \% \text { no water entry, } \\
33 \% \text { during dry out, } \\
10.1 \% \text { after dry out; } \\
1.29 \mathrm{~kW} / \mathrm{m} \text { during dry } \\
\text { out, } \\
<0.01 \mathrm{~kW} / \mathrm{m} \text { after } \\
\text { dryout; } \\
\text { Negligible T effect }\end{array}$ \\
\hline $\begin{array}{l}6.1 .8 \\
\text { HTOM, } \\
\text { LTOM }\end{array}$ & $3 D$ in-drift effects & $2 D$ versus $3 D$ & $3 D$ & $\begin{array}{l}\text { WP T (DB, AVG, } \\
\text { COOL) } \\
\text { postclosure peak }\end{array}$ & $\begin{array}{l}\text { HTOM } 26^{\circ} \mathrm{C} \text { range, } \\
\text { LTOM } 8^{\circ} \mathrm{C} \text { range }\end{array}$ \\
\hline
\end{tabular}

Source: Produced from results in Sections 5.3.1.4 and 5.3.2.4 of this document.

NOTES: $2 \mathrm{D}=$ two-dimensional; $3 \mathrm{D}=$ three-dimensional; $C F D=$ computational fluid dynamics; $\mathrm{DS}=$ drip shield; $\mathrm{DW}=\mathrm{drift}$ wall; HTOM = higher-temperature operating mode; $I N V_{L}=$ lower-center of invert; $I N V_{U}=$ upper layer of invert just outboard of the drip shield; LTOM = lower-temperature operating mode; NF = near-field; PWR = pressurized water reactor; $R H=$ relative humidity; $\mathrm{S}=$ saturation; SNFA = spent nuclear fuel assembly; $T$ = temperature; WP = waste package.

DB, AVG, COOL (WP) = Design Basis (11.8 kW initial), Average (7.4 kW average BWA), Cool (DOE high-level waste glass), $0.3 \mathrm{~kW}$ waste packages. 
Table 21 Bulk (Fracture) Permeability Values for Geologic Units used in the MSTH Model Analyses, Along with the Assumed Value of Sigma used in the Sensitivity Study of $\mathbf{k}_{b}$

\begin{tabular}{|c|c|c|c|c|}
\hline Unit & Permeability $(\mathbf{k}), \mathbf{m}^{2}$ & $\log (\mathbf{k})$ & sigma $\left(\log \mathbf{k}_{\mathbf{b}}\right)$ & two sigma $\left(\log \mathbf{k}_{\mathbf{b}}\right)$ \\
\hline tsw32 & $2.51 \mathrm{e}-12$ & -11.600 & 0.60 & 1.2 \\
\hline 33 & $8.79 \mathrm{e}-13$ & -12.056 & 0.60 & 1.2 \\
\hline 34 & $3.68 \mathrm{e}-13$ & -12.434 & 0.60 & 1.2 \\
\hline 35 & $2.38 \mathrm{e}-12$ & -11.623 & 0.60 & 1.2 \\
\hline $36 / 37$ & $1.38 \mathrm{e}-12$ & -11.860 & 0.60 & 1.2 \\
\hline
\end{tabular}

Source: Produced using files from Buscheck 2001 [DIRS 155012].

NOTE: No sigmas for tsw31 or 38/39. The high and low $k_{b}$ cases assumed one sigma and that the very high and very low $k_{b}$ cases assume two sigma deviation from the mean $k_{b}$ value.

Table 22 Lithophysal Unit Thermal Conductivity Values Used in the MSTH Model Sensitivity Analyses

\begin{tabular}{|c|c|c|c|c|c|c|}
\hline \multirow[b]{2}{*}{$K_{\text {th }}$ Case } & \multicolumn{2}{|c|}{ Lithophysal Porosity } & \multicolumn{2}{|c|}{$\begin{array}{c}\text { Saturated Thermal } \\
\text { Conductivity }(\mathrm{W} / \mathrm{m} \cdot \mathrm{K})\end{array}$} & \multicolumn{2}{|c|}{$\begin{array}{l}\text { Dry Thermal Conductivity } \\
(\mathrm{W} / \mathrm{m} \cdot \mathrm{K})\end{array}$} \\
\hline & $\begin{array}{l}\text { Lower } \\
\text { Lithophysal } \\
\text { Unit, Tptpll }\end{array}$ & $\begin{array}{l}\text { Upper } \\
\text { Lithophysal } \\
\text { Unit, Tptpul }\end{array}$ & $\begin{array}{l}\text { Lower } \\
\text { Lithophysal } \\
\text { Unit, Tptpll }\end{array}$ & $\begin{array}{c}\text { Upper } \\
\text { Lithophysal } \\
\text { Unit, Tptpul }\end{array}$ & $\begin{array}{l}\text { Lower } \\
\text { Lithophysal } \\
\text { Unit, Tptpll }\end{array}$ & $\begin{array}{c}\text { Upper } \\
\text { Lithophysal } \\
\text { Unit, Tptpul }\end{array}$ \\
\hline High $K_{\text {th }}$ & $0 \%$ & $5 \%$ & 2.02 & 2.13 & 1.54 & 1.43 \\
\hline Mean $K_{\text {th }}$ & $12.5 \%$ & $21.6 \%$ & 1.87 & 1.55 & 1.27 & 0.84 \\
\hline Low $K_{\text {th }}$ & $25 \%$ & $38 \%$ & 1.13 & 0.74 & 0.64 & 0.31 \\
\hline
\end{tabular}

Source: Adapted from BSC 2001 [DIRS 155008].

Table 23 Comparison of Relative Humidity Conditions on Drift Wall as Function of $\mathbf{K}_{t h}$ for the HigherThermal Operating Mode Case

\begin{tabular}{|l|c|c|c|c|c|}
\hline \multicolumn{1}{|c|}{$K_{\text {th }}$} & $\begin{array}{c}\text { RH at 50-60 } \\
\text { years post- } \\
\text { emplacement }\end{array}$ & $\begin{array}{c}\text { RH at } 400 \text { years } \\
\text { post- } \\
\text { emplacement }\end{array}$ & $\begin{array}{c}\text { RH at 1000 } \\
\text { years post- } \\
\text { emplacement }\end{array}$ & $\begin{array}{c}\text { Time to reach } \\
60 \% \text { Relative } \\
\text { Humidity }\end{array}$ & $\begin{array}{c}\text { Time to reach } \\
95 \% \text { Relative } \\
\text { Humidity }\end{array}$ \\
\hline High $K_{\text {th }}$ & $28 \%$ & $70 \%$ & $100 \%$ & 280 years & 700 years \\
\hline Mean $K_{\text {th }}$ & $20 \%$ & $60 \%$ & $95 \%$ & 400 years & 1000 years \\
\hline Low $K_{\text {th }}$ & $3-5 \%$ & $23 \%$ & $60 \%$ & 1000 years & 2000 years \\
\hline
\end{tabular}

Source: Produced using files from Buscheck 2001 [DIRS 155012]. 
Table 24 Lithophysal Porosity Values used in MSTH Model Sensitivity Analyses

\begin{tabular}{|c|c|c|}
\hline \multirow{2}{*}{ K $_{\text {th }}$ Case } & \multicolumn{2}{|c|}{ Lithophysal Porosity } \\
\cline { 2 - 3 } & $\begin{array}{c}\text { Lower } \\
\text { Lithophysal } \\
\text { Unit, Tptpll }\end{array}$ & $\begin{array}{c}\text { Upper Lithophysal } \\
\text { Unit, Tptpul }\end{array}$ \\
\hline High K th $_{\text {th }}$ & $0 \%$ & $5 \%$ \\
\hline Mean $\mathrm{K}_{\text {th }}$ & $12.5 \%$ & $21.6 \%$ \\
\hline Low $\mathrm{K}_{\text {th }}$ & $25 \%$ & $38 \%$ \\
\hline
\end{tabular}

Source: Produced using files from BSC 2001 [DIRS 155008], Table 24.

Table 25 Waste Package Peak Temperatures for the Three Lower-Temperature Operating Mode Sensitivity Cases

\begin{tabular}{|l|l|l|c|c|c|}
\hline \multicolumn{1}{|c|}{ Case } & Design Parameters & $\begin{array}{c}\text { Operational } \\
\text { Parameters }\end{array}$ & $\begin{array}{l}\text { Fraction of Waste } \\
\text { Packages with } \\
\text { Peak Temperature } \\
>855^{\circ} \mathrm{C} \text { (Full MSTH } \\
\text { Results) }\end{array}$ & $\begin{array}{c}\text { Peak Waste } \\
\text { Package } \\
\text { Temperature } \\
\text { (Full MSTH } \\
\text { Results) }\end{array}$ & $\begin{array}{c}\text { Peak Waste } \\
\text { Package } \\
\text { Temperature } \\
\text { (L5C3 MSTH } \\
\text { Results) }\end{array}$ \\
\hline $\begin{array}{l}\text { LTOM-PA } \\
\text { Base Case }\end{array}$ & $\begin{array}{l}5.5 \mathrm{~m} \text { drift diameter } \\
81 \mathrm{~m} \text { drift spacing } \\
21 \text { PWR Waste } \\
\text { packages }\end{array}$ & $\begin{array}{l}15 \mathrm{~m}^{3} / \mathrm{s} \text { ventilation } \\
\text { for } 300 \mathrm{yr}, \\
1.1 \mathrm{~m} \text { average WP } \\
\text { spacing }\end{array}$ & $1.7 \%$ & $86.0^{\circ} \mathrm{C}$ & $84.3^{\circ} \mathrm{C}$ \\
\hline $\begin{array}{l}\text { Wider Drift } \\
\text { Spacing }\end{array}$ & $\begin{array}{l}5.5 \mathrm{~m} \text { drift diameter } \\
97 \mathrm{~m} \text { drift spacing } \\
21 \text { PWR Waste } \\
\text { packages }\end{array}$ & $\begin{array}{l}15 \mathrm{~m}^{3} / \mathrm{s} \text { ventilation } \\
\text { for } 300 \mathrm{yr}, \\
0.1 \mathrm{~m} \text { WP spacing }\end{array}$ & Not calculated & Not calculated & $88.8^{\circ} \mathrm{C}$ \\
\hline $\begin{array}{l}\text { De-rated WP } \\
\text { Capacity }\end{array}$ & $\begin{array}{l}5.5 \mathrm{~m} \text { drift diameter } \\
81 \mathrm{~m} \text { drift spacing } \\
16 \text { PWR Waste } \\
\text { packages }\end{array}$ & $\begin{array}{l}15 \mathrm{~m}^{3} / \mathrm{s} \text { ventilation } \\
\text { for } 300 \mathrm{yr}, \\
0.1 \mathrm{~m} \text { WP spacing }\end{array}$ & $0 \%$ & $82.6^{\circ} \mathrm{C}$ & $81.7^{\circ} \mathrm{C}$ \\
\hline
\end{tabular}

Source: Produced using files from Buscheck 2001 [DIRS 155449].

NOTES: The three sensitivity cases use the full MSTH model and a simplified implementation of the MSTH model at a single location in the repository footprint.

PWR = pressurized water reactor; $M S T H=$ multiscale thermal-hydrologic; $\mathrm{WP}=$ waste package . 
Table 26 In-Drift Temperatures for the Higher-Temperature Operating Mode.

\begin{tabular}{|c|c|c|c|c|c|c|c|}
\hline \multirow[b]{2}{*}{ Location } & \multicolumn{7}{|c|}{ Temperature, ${ }^{\circ} \mathrm{C}$} \\
\hline & $10 \mathrm{yr}^{*}$ & $51 \mathrm{yr}$ & $65 y^{* *}$ & $200 \mathrm{yr}$ & $1000 \mathrm{yr}$ & $2000 \mathrm{yr}$ & $100,000 \mathrm{yr}^{\star \star \star *}$ \\
\hline \multicolumn{8}{|c|}{ Design Basis PWR WP } \\
\hline Waste Pkg Upper Half & 119.9 & 166.2 & 184.5 & 154.8 & 134.2 & 114.2 & 26.9 \\
\hline Waste Pkg Lower Half & 123.3 & 171.3 & 188.5 & 156.7 & 134.9 & 114.6 & 27.0 \\
\hline Drip Shield Top-Center & 107.8 & 150.9 & 173.3 & 149.0 & 131.9 & 112.8 & 26.8 \\
\hline Drip Shield Top-Corner & 105.2 & 147.5 & 170.7 & 147.7 & 131.5 & 112.6 & 26.8 \\
\hline Drip Shield Bottom-Corner & 106.7 & 149.8 & 172.5 & 148.5 & 131.8 & 112.7 & 26.8 \\
\hline Drift Wall Crown & 98.4 & 139.1 & 164.9 & 144.8 & 130.4 & 111.9 & 26.7 \\
\hline Drift Wall 1:30 & 98.8 & 139.8 & 165.4 & 145.0 & 130.4 & 111.9 & 26.7 \\
\hline Drift Wall Rib & 99.2 & 140.3 & 165.8 & 145.2 & 130.5 & 112.0 & 26.7 \\
\hline Drift Wall 4:30 & 99.3 & 140.4 & 165.9 & 145.3 & 130.5 & 112.0 & 26.8 \\
\hline Invert Top-Center & 119.6 & 166.7 & 185.1 & 154.9 & 134.2 & 114.2 & 26.9 \\
\hline Invert Top-Right & 101.5 & 143.2 & 167.9 & 146.2 & 130.9 & 112.2 & 26.8 \\
\hline \multicolumn{8}{|c|}{ Average WP (BWR) } \\
\hline Waste Pkg Upper Half & 112.1 & 156.6 & 177.3 & 152.0 & 133.2 & 113.7 & 26.9 \\
\hline Waste Pkg Lower Half & 114.6 & 160.3 & 180.2 & 153.3 & 133.7 & 114.0 & 26.9 \\
\hline Drip Shield Top-Center & 103.3 & 145.9 & 169.7 & 148.3 & 131.8 & 112.7 & 26.8 \\
\hline Drip Shield Top-Corner & 101.6 & 143.6 & 168.0 & 147.5 & 131.5 & 112.5 & 26.8 \\
\hline Drip Shield Bottom-Corner & 102.5 & 145.1 & 169.1 & 148.0 & 131.6 & 112.6 & 26.8 \\
\hline Drift Wall Crown & 96.1 & 137.0 & 163.5 & 145.3 & 130.6 & 112.0 & 26.7 \\
\hline Drift Wall 1:30 & 96.5 & 137.6 & 163.9 & 145.5 & 130.7 & 112.0 & 26.7 \\
\hline Drift Wall Rib & 96.7 & 137.8 & 164.0 & 145.6 & 130.7 & 112.0 & 26.7 \\
\hline Drift Wall $4: 30$ & 96.4 & 137.5 & 163.8 & 145.4 & 130.7 & 112.0 & 26.8 \\
\hline Invert Top-Center & 112.1 & 157.4 & 178.1 & 152.2 & 133.3 & 113.7 & 26.9 \\
\hline Invert Top-Right & 98.4 & 139.9 & 165.5 & 146.3 & 131.0 & 112.2 & 26.8 \\
\hline \multicolumn{8}{|c|}{ Cool WP (DHLW) } \\
\hline Waste Pkg Upper Half & 92.8 & 130.8 & 158.5 & 142.3 & 129.5 & 111.4 & 26.7 \\
\hline Waste Pkg Lower Half & 93.2 & 131.3 & 158.8 & 142.5 & 129.6 & 111.5 & 26.7 \\
\hline Drip Shield Top-Center & 91.7 & 129.6 & 157.7 & 142.0 & 129.4 & 111.4 & 26.7 \\
\hline Drip Shield Top-Corner & 91.4 & 129.2 & 157.4 & 141.9 & 129.4 & 111.3 & 26.7 \\
\hline Drip Shield Bottom-Corner & 91.1 & 128.8 & 157.2 & 141.8 & 129.3 & 111.3 & 26.7 \\
\hline Drift Wall Crown & 89.4 & 126.7 & 155.7 & 141.1 & 129.1 & 111.1 & 26.6 \\
\hline Drift Wall 1:30 & 89.5 & 126.9 & 155.9 & 141.2 & 129.1 & 111.2 & 26.6 \\
\hline Drift Wall Rib & 89.2 & 126.5 & 155.6 & 141.1 & 129.1 & 111.1 & 26.7 \\
\hline Drift Wall 4:30 & 88.5 & 125.5 & 154.8 & 140.7 & 129.0 & 111.1 & 26.7 \\
\hline Invert Top-Center & 93.0 & 131.1 & 158.8 & 142.5 & 129.6 & 111.5 & 26.7 \\
\hline Invert Top-Right & 89.7 & 127.1 & 156.0 & 141.3 & 129.2 & 111.2 & 26.7 \\
\hline
\end{tabular}

Source: Produced using files from Buscheck 2001 [DIRS 155449].

$$
\text { NOTE: } \begin{aligned}
* & =\text { Time of Preclosure Peak T } \\
* * & =\text { Time of Postclosure Peak T } \\
& =\text { Near-Ambient T. }
\end{aligned}
$$


Table 27 In-Drift Temperatures for the Lower-Temperature Operating Mode (Page 1 of 2)

\begin{tabular}{|c|c|c|c|c|c|c|c|}
\hline \multirow[b]{2}{*}{ Location } & \multicolumn{7}{|c|}{ Temperature, ${ }^{\circ} \mathrm{C}$} \\
\hline & $10 \mathrm{yr}^{*}$ & $301 \mathrm{yr}$ & $500 \mathrm{yr}$ & $780 \mathrm{yr}^{* *}$ & 1000 & 4000 & $100,000^{* * *}$ \\
\hline \multicolumn{8}{|c|}{ Design Basis PWR WP } \\
\hline Waste Pkg Upper Half & 84.6 & 76.2 & 97.4 & 98.4 & 97.0 & 73.8 & 26.5 \\
\hline Waste Pkg Lower Half & 86.8 & 77.9 & 98.6 & 99.3 & 97.7 & 74.1 & 26.5 \\
\hline Drip Shield Top-Center & 73.3 & 67.2 & 91.8 & 94.4 & 93.8 & 72.4 & 26.4 \\
\hline Drip Shield Top-Comer & 70.6 & 65.0 & 90.5 & 93.5 & 93.0 & 72.0 & 26.3 \\
\hline Drip Shield Bottom-Corner & 71.5 & 65.8 & 91.0 & 93.9 & 93.3 & 72.2 & 26.4 \\
\hline Drift Wall Crown & 63.2 & 59.3 & 87.1 & 91.2 & 91.1 & 71.2 & 26.2 \\
\hline Drift Wall 1:30 & 63.5 & 59.5 & 87.3 & 91.3 & 91.2 & 71.2 & 26.3 \\
\hline Drift Wall Rib & 64.1 & 60.0 & 87.5 & 91.5 & 91.4 & 71.3 & 26.3 \\
\hline Drift Wall 4:30 & 64.4 & 60.3 & 87.7 & 91.6 & 91.4 & 71.3 & 26.3 \\
\hline Invert Top-Center & 83.1 & 74.9 & 96.7 & 98.0 & 96.6 & 73.6 & 26.5 \\
\hline Invert Top-Right & 66.9 & 62.2 & 88.8 & 92.4 & 92.1 & 71.6 & 26.4 \\
\hline \multicolumn{8}{|c|}{ Average WP (BWR) } \\
\hline Waste Pkg Upper Half & 75.6 & 67.9 & 92.0 & 94.7 & 94.1 & 72.7 & 26.4 \\
\hline Waste Pkg Lower Half & 77.3 & 69.1 & 92.8 & 95.3 & 94.6 & 73.0 & 26.4 \\
\hline Drip Shield Top-Center & 68.2 & 62.6 & 88.8 & 92.4 & 92.2 & 71.8 & 26.3 \\
\hline Drip Shield Top-Corner & 66.7 & 61.5 & 88.1 & 91.9 & 91.8 & 71.6 & 26.3 \\
\hline Drip Shield Bottom-Corner & 67.4 & 62.0 & 88.4 & 92.2 & 92.0 & 71.7 & 26.3 \\
\hline Drift Wall Crown & 62.6 & 58.5 & 86.4 & 90.7 & 90.8 & 71.2 & 26.2 \\
\hline Drift Wall 1:30 & 62.8 & 58.7 & 86.5 & 90.8 & 90.8 & 71.2 & 26.2 \\
\hline Drift Wall Rib & 63.0 & 58.8 & 86.5 & 90.8 & 90.9 & 71.2 & 26.3 \\
\hline Drift Wall $4: 30$ & 62.9 & 58.7 & 86.5 & 90.8 & 90.9 & 71.2 & 26.3 \\
\hline Invert Top-Center & 74.9 & 67.3 & 91.8 & 94.6 & 93.9 & 72.7 & 26.4 \\
\hline Invert Top-Right & 64.3 & 59.8 & 87.1 & 91.2 & 91.2 & 71.4 & 26.3 \\
\hline \multicolumn{8}{|c|}{ Hot Gap } \\
\hline Drip Shield Top-Center & 67.8 & 61.9 & 88.0 & 91.8 & 91.7 & 71.6 & 26.3 \\
\hline Drip Shield Top-Corner & 66.8 & 61.2 & 87.6 & 91.5 & 91.5 & 71.5 & 26.3 \\
\hline Drip Shield Bottom-Corner & 67.2 & 61.5 & 87.8 & 91.7 & 91.6 & 71.5 & 26.3 \\
\hline Drift Wall Crown & 64.0 & 59.0 & 86.3 & 90.6 & 90.7 & 71.2 & 26.2 \\
\hline Dritt Wall 1:30 & 64.1 & 59.1 & 86.4 & 90.7 & 90.8 & 71.2 & 26.2 \\
\hline Drift Wall Rib & 64.1 & 59.1 & 86.4 & 90.7 & 90.8 & 71.2 & 26.3 \\
\hline Drift Wall 4:30 & 63.8 & 58.9 & 86.2 & 90.6 & 90.7 & 71.2 & 26.3 \\
\hline Invert Top-Center & 70.9 & 64.3 & 89.5 & 92.9 & 92.6 & 72.0 & 26.4 \\
\hline Invert Top-Aight & 64.9 & 59.8 & 86.8 & 91.0 & 91.0 & 71.3 & 26.3 \\
\hline
\end{tabular}


Table 27 (cont) In-Drift Temperatures for the Lower-Temperature Operating Mode (Page 2 of 2)

\begin{tabular}{|c|c|c|c|c|c|c|c|}
\hline \multicolumn{8}{|c|}{ Temperature, ${ }^{\circ} \mathrm{C}$} \\
\hline Location & $10 \mathrm{yr}^{*}$ & $301 \mathrm{yr}$ & $500 \mathrm{yr}$ & $780 \mathrm{yr}^{* *}$ & $1000 \mathrm{yr}$ & $4000 y$ & $100,000^{* * *}$ \\
\hline \multicolumn{8}{|c|}{ Cool Gap } \\
\hline Drip Shield Top-Center & 66.1 & 59.2 & 86.1 & 90.5 & 90.6 & 71.2 & 26.3 \\
\hline Drip Shield Top-Corner & 65.1 & 58.6 & 85.7 & 90.2 & 90.4 & 71.1 & 26.3 \\
\hline $\begin{array}{l}\text { Drip Shield Bottom- } \\
\text { Corner }\end{array}$ & 65.3 & 58.6 & 85.7 & 90.2 & 90.4 & 71.1 & 26.3 \\
\hline Drift Wall Crown & 62.5 & 57.0 & 84.8 & 89.6 & 89.9 & 70.9 & 26.2 \\
\hline Drift Wall $1: 30$ & 62.6 & 57.1 & 84.9 & 89.6 & 89.9 & 70.9 & 26.2 \\
\hline Drift Wall Rib & 62.6 & 57.0 & 84.8 & 89.6 & 89.9 & 70.9 & 26.3 \\
\hline Drift Wall 4:30 & 62.3 & 56.8 & 84.6 & 89.5 & 89.8 & 70.8 & 26.3 \\
\hline Invert Top-Center & 68.8 & 60.7 & 87.0 & 91.1 & 91.1 & 71.4 & 26.3 \\
\hline Invert Top-Right & 63.3 & 57.5 & 85.1 & 89.8 & 90.0 & 70.9 & 26.3 \\
\hline \multicolumn{8}{|c|}{ Cool WP (DHLW) } \\
\hline Waste Pkg Upper Half & 62.9 & 57.9 & 85.3 & 90.0 & 90.2 & 71.0 & 26.3 \\
\hline Waste Pkg Lower Half & 63.2 & 58.1 & 85.5 & 90.1 & 90.3 & 71.1 & 26.3 \\
\hline Drip Shield Top-Center & 61.9 & 57.4 & 85.1 & 89.8 & 90.1 & 71.0 & 26.3 \\
\hline Drip Shield Top-Comer & 61.7 & 57.3 & 85.0 & 89.7 & 90.0 & 70.9 & 26.3 \\
\hline $\begin{array}{l}\text { Drip Shield Bottom- } \\
\text { Corner }\end{array}$ & 61.5 & 57.1 & 84.9 & 89.7 & 90.0 & 70.9 & 26.3 \\
\hline Drift Wall Crown & 60.0 & 56.2 & 84.3 & 89.3 & 89.6 & 70.8 & 26.2 \\
\hline Drift Wall 1:30 & 60.1 & 56.2 & 84.4 & 89.3 & 89.7 & 70.8 & 26.2 \\
\hline Drift Wall Rib & 59.9 & 56.1 & 84.3 & 89.2 & 89.6 & 70.8 & 26.2 \\
\hline Drift Wall 4:30 & 59.5 & 55.8 & 84.1 & 89.1 & 89.5 & 70.7 & 26.3 \\
\hline Invert Top-Center & 63.1 & 58.1 & 85.5 & 90.1 & 90.3 & 71.1 & 26.3 \\
\hline Invert Top-Right & 60.3 & 56.4 & 84.4 & 89.3 & 89.7 & 70.8 & 26.3 \\
\hline
\end{tabular}

Source: Produced using files from Buscheck 2001 [DIRS 155449].

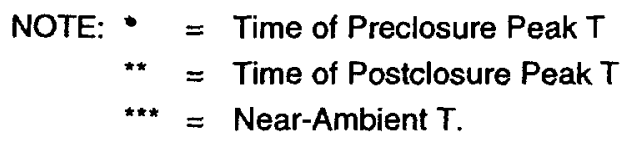

